\title{
Structural and Electronic Investigation of Strongly Correlated Transition Metal Oxide Perovskite Thin Films and Interfaces using In-situ Transmission Electron Microscopy
}

\author{
Dissertation \\ for the award of the degree \\ "Doctor rerum naturalium" (Dr.rer.nat.) \\ of the Georg-August-Universität Göttingen \\ within the doctoral program of \\ the Faculty of Physics \\ of the Georg-August University School of Science (GAUSS) \\ submitted by \\ Tobias Meyer \\ from Peine
}

Göttingen, 2020 
Thesis Committee:

Prof. Dr. Michael Seibt

IV. Physikalisches Institut, Georg-August-Universität Göttingen

Prof. Dr. Christian Jooß

Institut für Materialphysik, Georg-August-Universität Göttingen

Prof. Dr. Peter Blöchl

Institut für Theoretische Physik, Technische Universität Clausthal

Institut für Theoretische Physik, Georg-August-Universität Göttingen

Members of the Examination Board:

Reviewer: Prof. Dr. Michael Seibt

IV. Physikalisches Institut, Georg-August-Universität Göttingen

Second Reviewer: Prof. Dr. Christian Jooß

Institut für Materialphysik, Georg-AugustUniversität Göttingen

Further members of the Examination Board:

Prof. Dr. Peter Blöchl

Institut für Theoretische Physik, Technische Universität Clausthal

Institut für Theoretische Physik, Georg-August-Universität Göttingen

Prof. Dr. Hans-Christian Hofsäss

II. Physikalisches Institut, Georg-August-Universität Göttingen

Prof. Dr. Claus Ropers

IV. Physikalisches Institut, Georg-August-Universität Göttingen

Max-Planck-Institut für biophysikalische Chemie, Göttingen

Prof. Dr. Cynthia A. Volkert

Institut für Materialphysik, Georg-August-Universität Göttingen

Date of the oral examiniation:

21.09.2020 


\section{Contents}

1 Introduction $\quad 1$

2 Physical Background 5

2.1 Transition Metal Oxide Perovskites . . . . . . . . . . 5

2.1.1 Structural Properties . . . . . . . . . . . . 5

2.1.2 Influence of Octahedral Tilt and Distortion on Pbnm

Lattice Parameters . . . . . . . . . . . 8

2.1.3 Significance of the Structural Degrees of Freedom in $\mathrm{Pr}_{1-x} \mathrm{Ca}_{x} \mathrm{MnO}_{3}(\mathrm{PCMO}) \ldots \ldots \ldots$

2.1.4 Correlation Phenomena . . . . . . . . . . 16

2.1.5 Ordered Phases in PCMO . . . . . . . . . . . 18

2.1.6 Influence of Ordering on the Photovoltaic Activity of PCMO thin films on $\mathrm{SrTi}_{1-y} \mathrm{Nb}_{y} \mathrm{O}_{3}$ (STNO) . . . . 20

2.2 Photovoltaic Effect in Classical Semiconductors . . . . . . . 21

2.2.1 The $p$ - $n$ Junction . . . . . . . . . . . . . . . . 21

2.2.2 Excess Charge Carrier Generation and Recombination 23

2.2.3 Photovoltaic Effect in the One Diode Model . . . . . . 25

3 Experimental Methods $\quad 29$

3.1 Scanning Electron Microscopy (SEM) . . . . . . . . . . . . 29

3.1.1 General Setup of an SEM . . . . . . . . . . . 29

3.1 .2 Focused Ion Beam (FIB) . . . . . . . . . . . 30

3.1.3 Electron Beam Induced Current (EBIC) . . . . . . . . 30

3.2 Transmission Electron Microscopy (TEM) . . . . . . . . . . 31

3.2.1 Conventional and High-resolution TEM . . . . . . . . 31

3.2.2 Scanning Transmission Electron Microscopy (STEM) . 32

3.2.3 Electron Energy Loss Spectroscopy (EELS) and Energy Filtering . . . . . . . . . . . . . . 32

3.2.4 Energy-dispersive X-ray Spectroscopy . . . . . . . 33 
3.2.5 Four-dimensional STEM (4D-STEM) . . . . . . . 34

3.2.6 Environmental TEM . . . . . . . . . . . . . . 34

3.2.7 In-situ Biasing, Heating and Cooling . . . . . . . . . 35

3.2 .8 STEM-EBIC . . . . . . . . . . . . . 35

3.3 Used Experimental Setups . . . . . . . . . . . . . . 36

4 Method Development $\quad 37$

4.1 TEM Sample Preparation . . . . . . . . . . . . . . 37

4.1 Planview Preparation . . . . . . . . . . . . 37

4.1.2 Cross-sectional Preparation for In-situ Heating and Biasing . . . . . . . . . . . . 46

4.2 Probe Shaping via a Custom Condensor Aperture . . . . . . . 48

4.3 Implementation of 4D-STEM . . . . . . . . . . . 50

4.3.1 4D-STEM Acquisition . . . . . . . . . . 50

4.3.2 4D-STEM Analysis . . . . . . . . . . . . . . . 55

4.4 Synchronisation and Quantification of STEM Signals . . . . 66

5 Inverse Modelling of Octahedral Tilts and Distortions in Bulk PCMO

6 Exploring the Phase Diagram of PCMO Thin Films $\quad 77$

6.1 Charge Ordering for $x=0.34 \ldots \ldots \ldots$. . . . . 77

6.2 Pseudo-Tetragonal Transition for $x=0.1 \ldots \ldots$. . . . 84

6.3 Low Temperature Investigations for $x=0.1 \ldots \ldots$. . . . . 94

7 Scanning Transmission Electron Beam Induced Current 99

7.1 Applying the One Diode Model to Electron Transparent Lamel-

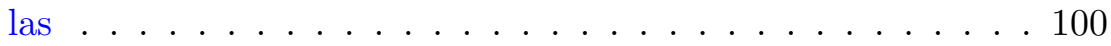

7.2 STEM-EBIC of Misfit Dislocations at PCMO-STNO Interfaces103

7.3 Extraction of Bulk Diffusion Lengths and Surface Layer Properties . . . . . . . . . . . . . . 111

7.4 In-situ Biased STEM-EBIC of PCMO $(x=0.34) \ldots 116$

7.5 STEM-EBIC in a Textured Silicon Solar Cell . . . . . . . . . 119

7.6 Atomically Resolved STEM-EBIC . . . . . . . . . . . 127

8 Summarising Discussion and Outlook 133

8.1 Method Development . . . . . . . . . . . . . . 133

8.2 Phase Diagram of $\mathrm{Pr}_{1-x} \mathrm{Ca}_{x} \mathrm{MnO}_{3}$ (PCMO) Thin Films . . . 134

8.3 Scanning Transmission Electron Beam Induced Current (STE$\mathrm{BIC}) \ldots \ldots \ldots \ldots \ldots \ldots \ldots \ldots$ 
A Appendix 139

A.1 Rotation Matrix Convention . . . . . . . . . . . . . . 139

A.2 Uniqueness of the Pbnm Rotation Axis . . . . . . . . . . 139

A.3 4D-STEM Analysis: Virtual Masking . . . . . . . . . . . . . . 140

A.4 4D-STEM Analysis: Bragg Analysis . . . . . . . . . . . . . 141

A.5 4D-STEM Analysis: First Moment . . . . . . . . . . . . 142

A.6 4D-STEM Analysis: Virtual DPC . . . . . . . . . . . . . 143

A.7 4D-STEM Analysis: Edge Detection . . . . . . . . . . . . 144

A.8 4D-STEM Analysis: FEM . . . . . . . . . . . . . . 145

A.9 ADC Calibration . . . . . . . . . . . . . . . 146

A.10 A posteriori synchronization of scanning transmission electron microscopy signals with kilopixel per second acquisition rates (Supplementary Material) . . . . . . . . . . . . 147

A.11 Macroscopic Perovskite Thin Film Samples . . . . . . . . . 157

A.12 Charge Ordering in PCMO $(x=0.34) \ldots \ldots . . . . .158$

A.13 Domain Boundaries in PCMO $(x=0.1) \ldots . . . . . .160$

A.14 Determination of Decay Lengths from EBIC Profiles . . . . . 161

A.15 Interface Localisetion of PCMO-STNO Heterojunctions . . . 163

A.16 Atomically Resolved EBIC: Domain Assignment . . . . . . . 164

$\begin{array}{lc}\text { References } & 183\end{array}$ 


\section{Chapter 1}

\section{Introduction}

Discussing the necessity as well as possible details of global strategies to reduce and eventually eliminate the anthropogenic climate change (ACC) is a delicate matter, which easily leads to statements based on ressentiments rather than on scientific facts. Indeed, public polls revealed the volatility of individual beliefs in the existence of ACC correlating with short-term weather phenomena [1] well after a scientific consensus about its impact was found [2-5]. Naturally, the models presented in the cited references do not cover all facets of the cybernetic global system at once and the assessment of resulting forecast uncertainties is part of the careful work of colleagues [6, 7]. However, the included and certainly possible scenario of turning the Earth in an increasingly hostile planet appears to be an unreasonably high stake when betting on the future.

Consequently, in order to reduce the emission of greenhouse gases being indisputably linked to global warming $[7,8]$, progress in sustainable energy sources as well as their actual usage is indispensable. In essence, establishing a prevailing renewable energy supply is a threefold problem as primary conversions need to be followed by storage and transport in order to bridge temporal as well as spatial source heterogeneities [9]. One candidate to master the former challenge is solar energy conversion, which has recently gained a lot in global energy market share as module efficiencies resp. prices are constantly rising resp. falling $[10,11]$. In detail, both the optimisation of established solar cells, i.e. most prominently silicon modules, as well as the inclusion of innovative concepts and materials is pursued. The latter approach is also referred to as third-generation photovoltaics and aims for solar cell efficiencies beyond the famous Shockley-Queisser limit [12], which describes the theoretical thermodynamic conversion limit under the assump- 
tion of transmission of sub-bandgap photons and complete thermalisation of hot charge carriers before power extraction. A particularly promising example, in which these assumptions are no longer valid, is given by the material class of organic halide perovskites showing an extraordinarily fast increase of related efficiencies over the past years [13-15].

In this work, the inorganic counterpart of transition metal oxide perovskites will be the main subject of study. Certainly, currently achieved solar energy conversion efficiencies in this material class are significantly lower compared with organic halides [16-18]. However, because of their rich phase diagrams emerging due to strong correlations [19-22] they serve as a wellsuited model system to study underlying mechanisms (lifting the previously mentioned Shockley-Queisser limit) on a fundamental level. Importantly, this statement is not limited to the context of photovoltaics, but holds also for additional fields such as the study of catalysis [23-25] or resistive random access memory (RRAM) [26].

In more detail, this dissertation focuses on the structural and electronic investigation of transition metal oxide perovskite thin films, being typically the basis of technological devices [26]. It includes significant contributions to the phase diagram of $\operatorname{Pr}_{1-x} \mathrm{Ca}_{x} \mathrm{MnO}_{3}$ epitaxial layers - grown on $\mathrm{SrTi}_{1-y} \mathrm{Nb}_{y} \mathrm{O}_{3}$ substrates - in doping and temperature regimes where ordered phases occur due to correlative exchange interactions of lattice, orbital, and spin degrees of freedom [20]. Importantly, these ordered phases have been demonstrated to correlate with an enhanced photovoltaic acitvity $[17,18,27,28]$ emphasizing the importance of such studies in the context of solar energy conversion. In fact, as nicely described in the cited references, the underlying mechanism of the enhanced photovoltaic activity was found to be a prolonged lifetime of hot carriers due to phonon interactions and, thus, reaches beyond the assumptions of the Shockley-Queisser limit. Consequently, the materials in question are well-suited to explore fundamental processes in third generation photovoltaics.

In order to study the mentioned phase transitions in thin films as well as electric properties relevant for solar energy conversion such as the excess charge carrier diffusion length, which happens to be located on the nanoscale [29], high-resolution techniques are needed. Therefore, the transmission electron microscope is employed enabling for versatile and highlyresolved real and reciprocal space signal extraction as well as a large variety of in-situ techniques, e.g. heating, cooling, biasing, and environmental control. In fact, facilitated by the outstanding advances in scientific instrumentation, such in-situ methods are ever-increasingly applied on the microand nanoscale and successfully correlated to macroscopic physical, chemical, 
and biological properties [30-33]. In this study, in-situ heating, cooling, biasing, and environmental control are combined with established techniques such as selective area electron diffraction [34] and electron energy loss spectroscopy [35] as well as with recently emerging methods like four-dimensional scanning transmission electron microscopy [36] and scanning transmission electron beam induced current [29]. Additionally, a substantial part of this thesis focuses on further developments of the latter techniques.

Selected highlights are the successful extraction of ordering parameters as well as critical temperatures of phase transitions in $\operatorname{Pr}_{1-x} \mathrm{Ca}_{x} \mathrm{MnO}_{3}$ during heating (in a gaseous environment) and cooling. The observed transitions, i.e. charge ordering for $x=0.34$ and an orthorhombic to pseudotetragonal (or cubic) transition for $x=0.1$, are discussed thoroughly in the context of correlation phenomena and photovoltaic activity and differences to the bulk such as decreased critical temperatures will be pointed out. Furthermore, a structural model is presented linking atomic configurations with the material's lattice parameters. In addition, experimental and modelling advances in the field of scanning transmission electron beam induced current are demonstrated enabling the observation of diffusion and recombination properties of excess charge carriers in perovskites on the nanoscale as well mapping of a sub-0.1 ppm concentration line of boron in a textured silicon solar cell. Lastly, first interpretations of atomic modulations in electron beam induced current signals are presented. 


\section{Chapter 2}

\section{Physical Background}

In this chapter, the main topics of this dissertation shall be discussed on a theoretical basis introducing relevant formalisms and equations. It is divided into two major parts: Firstly, an introduction on transition metal oxide perovskites (TMOPs) will be given focusing on the manganite $\mathrm{Pr}_{1-x} \mathrm{Ca}_{x} \mathrm{MnO}_{3}$ (PCMO) and related correlation phenomena. Secondly, a brief summary of classical semiconductor physics linked to photovoltaic applications will be presented.

\subsection{Transition Metal Oxide Perovskites}

As mentioned above, this section serves as an introduction to the main concepts being relevant in the context of TMOPs. Due to their strong correlation, many aspects including lattice, orbital, and spin effects have to be accounted for to create a complete and fully consistent picture. Nevertheless, in many cases, simplified models suffice to describe underlying phenomena on a qualitative level which is why several references to additional literature will be given throughout this section while discussing only the tip of the perovskite iceberg.

\subsubsection{Structural Properties}

The term perovskite includes both materials obtaining the ideal cubic structure with formula $A B C_{3}$ shown in Fig. 2.1 [37] as well as those exhibiting characteristic deviations, which will be partially described in this subsection. While the $B$ - and $C$-sites are occupied by a transition metal resp. oxygen (in the case of TMOPs), $A$ is frequently chosen as a rare or alkaline earth metal, 
or in cases of alloyed sites as a mixture of both. Most of the dominant structural deviations mentioned above can be understood best by considering the $\mathrm{BO}_{6}$ octahedron spanned by the oxygen atoms surrounding the $B$-site, which is visualised by the purple surfaces in Fig. 2.1. Importantly, each oxygen belongs to two corner-sharing octahedra leading to collaborative motions in the material. Fig. 2.2 illustrates two prominent examples of collaborative, structural deviations from the ideal perovskite: On the left side, the octahedra are rigidly rotated while on the right side the $B$-O distances are chosen anisotropically, i.e. the octahedra are distorted. For the sake of clarity, the

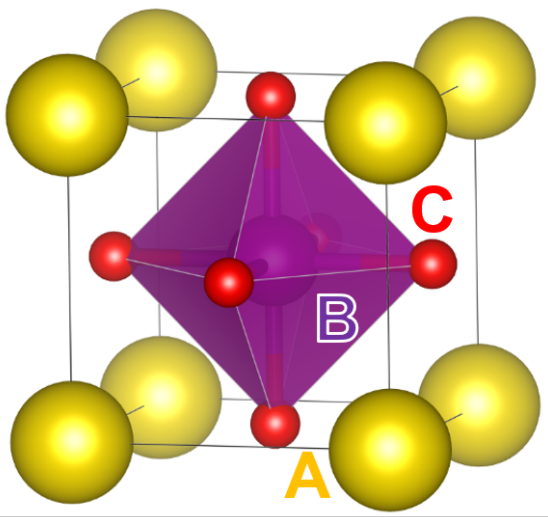

Figure 2.1: Ideal cubic perovskite structure with atom species $A, B$, and $C$ occupying the primitive, bodycentered, and face-centered sites. The purple surfaces illustrate the octahedron spanned by the six $\mathrm{C}$ atoms with $\mathrm{B}$ in its center. A-site atoms are omitted. Still, it is important to mention that the distance between neighbouring oxygen and A-site atoms as well as the latter's configuration number is reduced due to the tilt. Thus, it serves as a possible relaxation of mismatch in the averaged ionic radii $\left(\left\langle r_{A}\right\rangle,\left\langle r_{B}\right\rangle\right.$, and $\left.\left\langle r_{\mathrm{O}}\right\rangle\right)$ to fit in the cubic structure, which is described by the Goldschmidt tolerance factor defined as [38]

$$
T_{\mathrm{G}}=\frac{\left\langle r_{A}\right\rangle+r_{\mathrm{O}}}{\sqrt{2} \cdot\left(\left\langle r_{B}\right\rangle+r_{\mathrm{O}}\right)} .
$$

In fact, physical properties, e.g. metal-to-insulator or magnetic transition temperatures, can be predicted by $T_{\mathrm{G}}<1$ for some choices of $A$ and $B$ [39], but counterexamples exist as well [40]. Please note that the tilt pattern shown on the left side in Fig. 2.2 corresponds to $a^{-} a^{-} c^{+}$in the so-called Glazer notation and is only one among many others leading to different symmetries, i.e. space groups, as summarised in [41]. Similarly, the collaborative distortion pattern is not unique and has been chosen, in accordance with $\mathrm{PCMO}$, to be alternating in the $a b$-plane and constant along the perpendicular $c$-direction. In this particular case, two important changes happen compared to the cubic structure: Firstly, as shown in the top left image, only every other octahedra is equivalent in $c$-direction leading to a doubling 

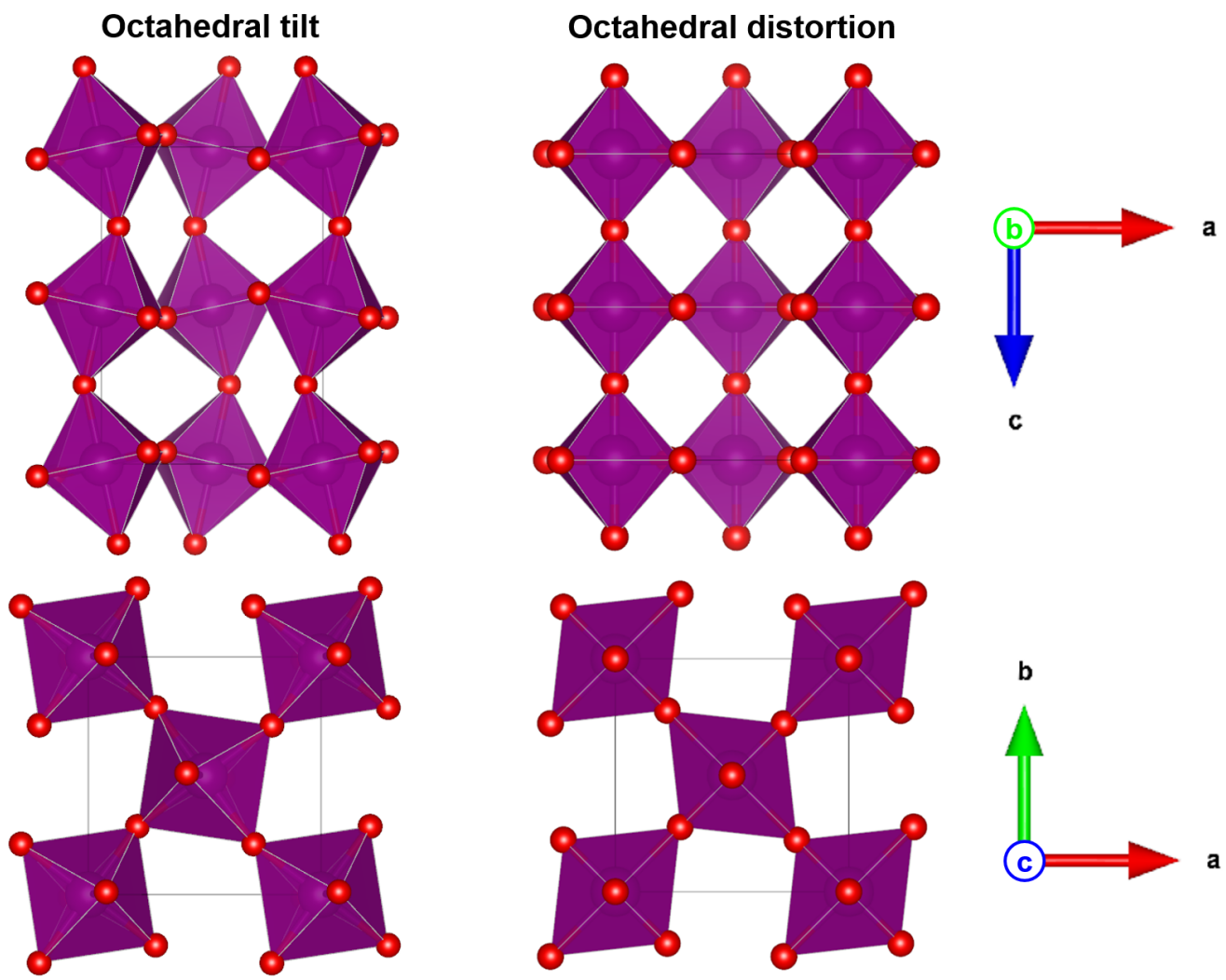

Figure 2.2: Visualisation of collaborative tilt (left) and distortion (right) in corner-shared networks of $\mathrm{BO}_{6}$ octahedra. Red resp. purple atoms represent oxygen resp. the central $B$-site atoms. The exemplary tilt corresponds to $a^{-} a^{-} c^{+}$in the Glazer notation while the $B$-O distance anisotropy is alternating in the $a b$-plane and constant along the $c$-direction. 
of the lattice constant. Please note that this effect is purely due to the tilt as the $B$-O distance is constant along $c$. Secondly, as shown in the bottom images, both the tilting as well as the alternating $B$-O distances in the $a b$ plane lead again to non-equivalent neighbouring sites. Here, in contrast to the $c$-direction, following the face-diagonal of the previously cubic structure leads to the closest equivalent sites and thus to an approximately $45^{\circ}$ rotation and scaling by $\sqrt{2}$ of $a$ and $b$ compared to the ideal perovskite. The resulting space group is orthorhombic, denoted as Pbnm in the HermannMauguin convention [41], and has been chosen here since it is obtained by PCMO, which is of major interest in this work. Nevertheless, it is worth mentioning that as a consequence of different tilt and distortion patterns different structures can be found in TMOPs as well, e.g. cubic, tetragonal, rhombohedral, or monoclinic [42-45]. In addition, ordering of different types of $A$-sites in so-called double perovskites has been reported [46], as well as alternating stacking of perovskite and A-O-layers leading to the definition of Ruddlesdon-Popper (RP) perovskite phases [47].

\subsubsection{Influence of Octahedral Tilt and Distortion on Pbnm Lattice Parameters}

In the past, various studies have been conducted to illuminate the symmetry of the involved $\mathrm{BO}_{6}$ octahedra in TMOPs: In [41], where the Glazer notation was presented first, different tilt patterns and their corresponding space groups were classified. In addition, the problem of ambiguous combinations of distortions and tilts leading to the same lattice parameters was raised. Building on that, it was shown in [48] that certain tilt patterns can only be realised in connected 3D networks of octahedra if the latter are non-rigid. For the sake of a clear terminology, such non-rigid behaviour will subsequently be referred to as a distortion if the $B$-O distances are anisotropic and a twisting if one of the $\mathrm{O}-B-\mathrm{O}$ bonding angles (of non-opposed oxygen) differs from $90^{\circ}$. According to [48], in the case of a $a^{-} a^{-} c^{+}$tilt pattern resulting in the herein considered $\mathrm{Pbnm}$ space group, neither a distortion nor a twisting is per se necessary. Indeed, [49] confirms that no significant twisting can be found experimentally for $B=\mathrm{Mn}$ if the ionic radius of $A$ is below $1.16 \AA$, i.e. in the case of PCMO. However, distortions are frequently observed [20]. Relying on the absence of twisting and a Pbnm space group, existing general geometrical considerations to describe the lattice parameters in perovskites by means of octahedral degrees of freedom can be specialised as it has been done in [50]. Here, a different parametrisation, i.e. a single

rotational operation along an a priori free axis shall be used to express the 


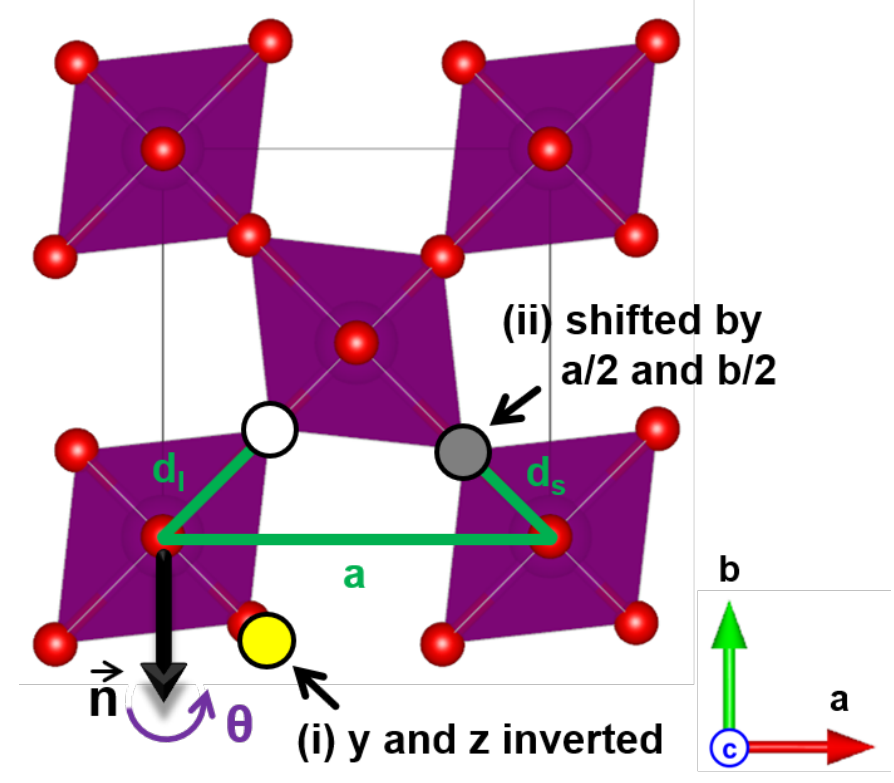

Figure 2.3: Illustration of the two different paths leading from one oxygen position (white circle) to another (grey circle): Following the Pbnm symmetry operations, subsequent inversion of $y$ and $z$ (i) as well as shifting by $a / 2$ and $b / 2$ (ii) has to lead to the same result as translating along the $B$-O bonds resp. the unit cell edge as marked by the green path.

lattice parameters instead of subsequently applied rotations.

The underlying concept of how to link lattice parameters and structural modes is visualised in Fig. 2.3: Assuming a Pbnm space group as well as rotated and distorted octahedra, there are two different paths to get from the white to grey oxygen position. Firstly, the symmetry operation $(x, y, z) \rightarrow(0.5+x, 0.5-y,-z)$ can be used, which is split in its inversion and translation part in the illustration. Secondly, translating along the $B-O$ bonds as well as the edge of the unit cell has to lead to the same result. Mathematically, by exploiting that the lower left and lower right octahedra in Fig. 2.3 are equivalent and rotated likewise, this can be described as:

$$
\left(\begin{array}{c}
a / 2+d_{\mathrm{l}} / \sqrt{2}\left(R_{11}(\vec{n}, \theta)+R_{12}(\vec{n}, \theta)\right) \\
b / 2-d_{\mathrm{l}} / \sqrt{2}\left(R_{21}(\vec{n}, \theta)+R_{22}(\vec{n}, \theta)\right) \\
-d_{\mathrm{l}} / \sqrt{2}\left(R_{31}(\vec{n}, \theta)+R_{32}(\vec{n}, \theta)\right)
\end{array}\right)=\left(\begin{array}{c}
a \\
0 \\
0
\end{array}\right)+\mathbf{R}(\vec{n}, \theta)\left(\begin{array}{c}
-d_{\mathrm{s}} / \sqrt{2} \\
d_{\mathrm{s}} / \sqrt{2} \\
0
\end{array}\right) .
$$


Here, $R_{i j}(\vec{n}, \theta)$ denote the components of a $3 \mathrm{D}$ rotation matrix $\mathbf{R}(\vec{n}, \theta)$ along the normal vector $\vec{n}$ with rotation angle $\theta$ and $d_{\mathrm{l}}$ (long), $d_{\mathrm{s}}$ (short), and $d_{\mathrm{m}}$ (medium) the anisotropic $B$-O distances pointing along [110], [110], and [001] in the lower left ocetahedron of Fig. 2.3. Rearranging Eq. (2.2) and using the definition of $\mathbf{R}(\vec{n}, \theta)$ given in Eq. (A.1), the following conditions can be derived:

$$
\begin{aligned}
a & =\sqrt{2}\left(d_{\mathrm{l}}+d_{\mathrm{s}}\right)\left(\cos \theta+n_{1}^{2}(1-\cos \theta)\right) \\
& +\sqrt{2}\left(d_{\mathrm{l}}-d_{\mathrm{s}}\right)\left(n_{1} n_{2}(1-\cos \theta)-n_{3} \sin \theta\right), \\
b & =\sqrt{2}\left(d_{\mathrm{l}}+d_{\mathrm{s}}\right)\left(\cos \theta+n_{2}^{2}(1-\cos \theta)\right) \\
& +\sqrt{2}\left(d_{\mathrm{l}}-d_{\mathrm{s}}\right)\left(n_{2} n_{1}(1-\cos \theta)+n_{3} \sin \theta\right), \\
0 & =\left(d_{1}+d_{\mathrm{s}}\right)\left(n_{3} n_{2}(1-\cos \theta)+n_{1} \sin \theta\right) \\
& +\left(d_{\mathrm{l}}-d_{\mathrm{s}}\right)\left(n_{3} n_{1}(1-\cos \theta)-n_{2} \sin \theta\right) .
\end{aligned}
$$

In addition, as the rotation axis is normalised and the oxygen atoms pointing along [001] have to be located in the $z=c / 4$ mirror plane of the Pbnm space group to maintain the stoichiometry, the following two conditions must be satisfied as well:

$$
\begin{aligned}
c & =4 d_{\mathrm{m}}\left(\cos \theta+n_{3}^{2}(1-\cos \theta)\right), \\
n_{3} & =\sqrt{1-n_{1}^{2}-n_{2}^{2}} .
\end{aligned}
$$

Please note that choosing $n_{3}$ to be positive imposes no loss of generality as long as the sign of $\theta$ is not fixed. While Eq. (2.3), (2.4), and (2.6) define the lattice parameters as functions of the $B$-O distances, $\vec{n}$ and $\theta$, the conditions given in Eq. (2.5) and (2.7) limit the choice of $\vec{n}$. In fact, for a given tuple $\left(n_{2}, \theta, d_{1}, d_{\mathrm{s}}\right), n_{1}$ and $n_{3}$ can be defined as:

$$
\begin{aligned}
& n_{1}\left(n_{2}, \theta, d_{\mathrm{l}}, d_{\mathrm{s}}\right) \in\left\{x: f\left(x, n_{2}, \theta, d_{\mathrm{l}}, d_{\mathrm{s}}\right)=0\right\}, \\
& n_{3}\left(n_{2}, \theta, d_{\mathrm{l}}, d_{\mathrm{s}}\right):=\sqrt{1-n_{1}\left(n_{2}, \theta, d_{\mathrm{l}}, d_{\mathrm{s}}\right)^{2}-n_{2}^{2}},
\end{aligned}
$$

with

$$
\begin{aligned}
f\left(x, n_{2}, \theta, d_{\mathrm{l}}, d_{\mathrm{s}}\right) & =\left(d_{\mathrm{l}}+d_{\mathrm{s}}\right)\left(n_{2} \sqrt{1-x^{2}-n_{2}^{2}}(1-\cos \theta)+x \sin \theta\right) \\
& +\left(d_{\mathrm{l}}-d_{\mathrm{s}}\right)\left(x \sqrt{1-x^{2}-n_{2}^{2}}(1-\cos \theta)-n_{2} \sin \theta\right) .
\end{aligned}
$$




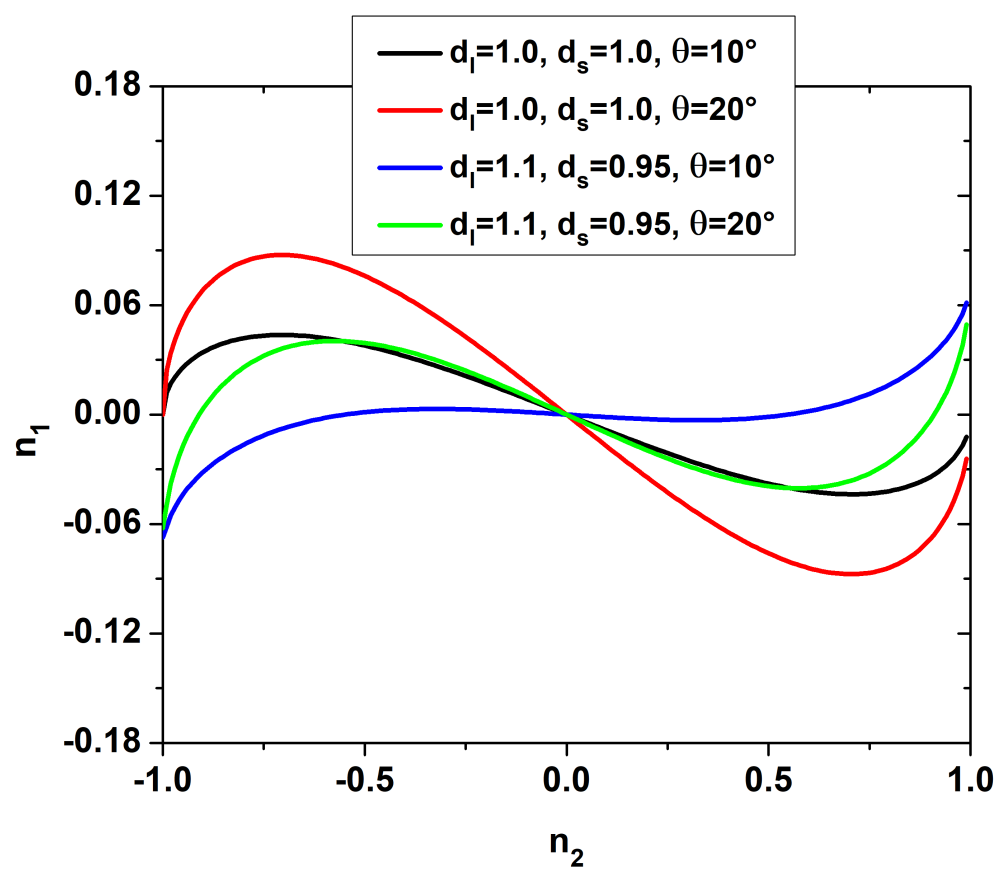

Figure 2.4: Values for $n_{1}\left(n_{2}, \theta, d_{1}, d_{\mathrm{s}}\right)$ as a function of $n_{2}$ for different choices of the remaining parameters obtained with Newton's method to solve for roots of Eq. (2.10).

Even though no mathematical proof will be given in this thesis, Fig. A.1 suggests that the choice of $n_{1}$, and thus also of a positive $n_{3}$, is unique over a wide range of $\left(n_{2}, \theta, d_{\mathrm{l}}, d_{\mathrm{s}}\right)$ and the corresponding values will subsequently be determined numerically using Newton's method. Fig. 2.4 shows $n_{1}$ as a function of $n_{2}$ for different choices of the remaining parameters confirming that there are indeed solutions for values $n_{2} \in(-1+\epsilon, 1-\epsilon)$ where $\epsilon \lesssim 0.001$ depends on the remaining parameters and originates since solutions of Eq. (2.5) lead to complex values for $n_{3}$ in Eq. (2.7).

As a matter of fact, these considerations already lead to a reduction of the positional degrees of freedom in the Pbnm unit cell since the lattice constants as well as all positions of $B$ and $\mathrm{O}$ atoms are expressed by the five parameters $n_{2}, \theta, d_{1}, d_{\mathrm{s}}$, and $d_{\mathrm{m}}$. In contrast, in the general case including twisted octahedra, the lattice constants are independent of the five positional coordinates of $B$ and $\mathrm{O}$. Please note that in either cases there are still two degrees of freedom of the herein omitted $A$-site atoms. 


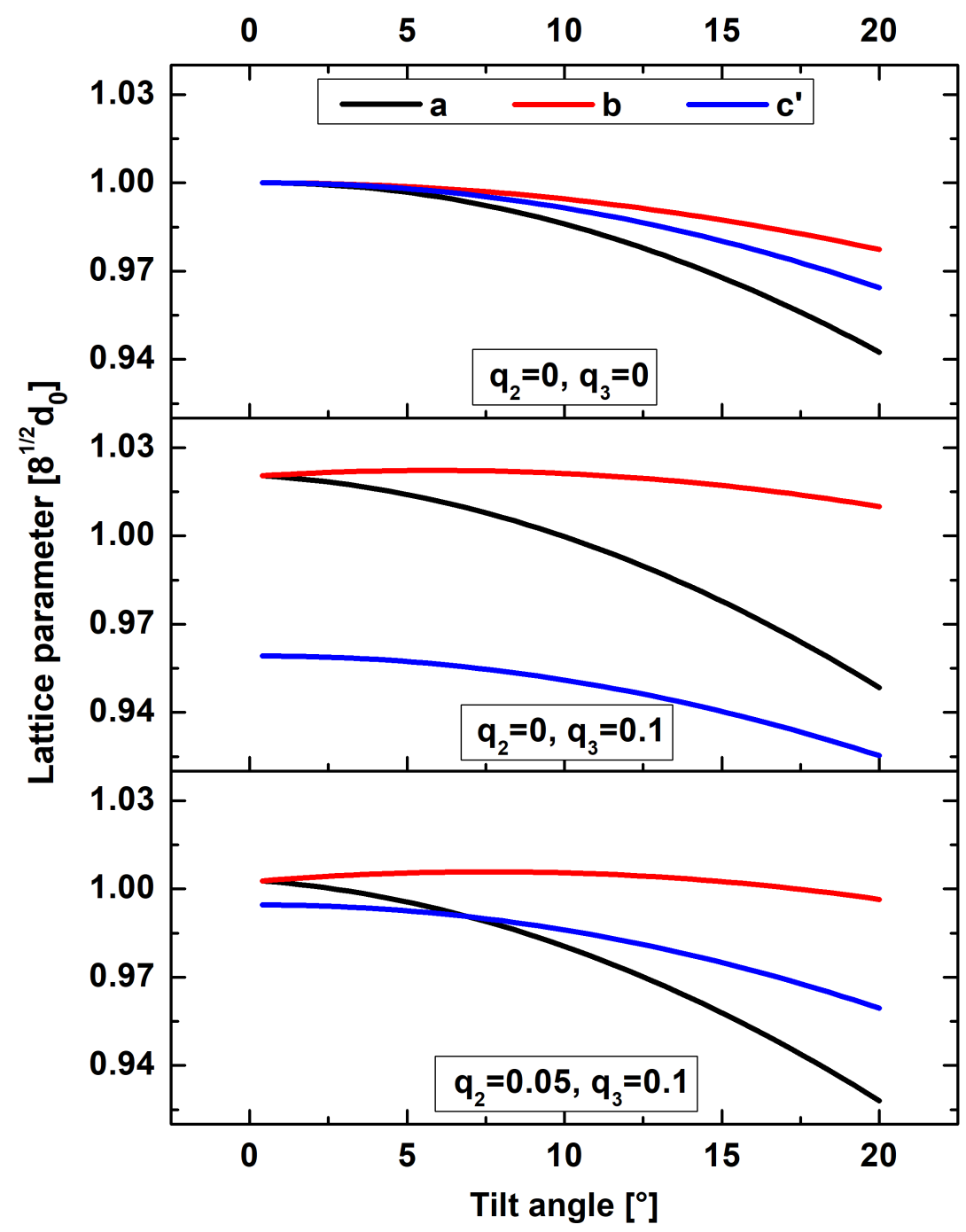

Figure 2.5: Lattice parameters $a, b$, and $c^{\prime}$ as a function of the tilt angle $\theta$ for (top) $q_{2}=q_{3}=0$, (center) $q_{2}=0, q_{3}=0.1$, (bottom) $q_{2}=0.05, q_{3}=0.1$. 
Lastly, a different basis shall be chosen to express $d_{\mathrm{l}}, d_{\mathrm{s}}$, and $d_{\mathrm{m}}$ with their mean value $d_{0}$ as well as two relative static distortions $q_{2}$ and $q_{3}$ :

$$
\begin{aligned}
d_{1} & =d_{0}\left(1+\frac{2}{\sqrt{6}} q_{3}\right) \\
d_{\mathrm{s}} & =d_{0}\left(1-\frac{1}{\sqrt{2}} q_{2}-\frac{1}{\sqrt{6}} q_{3}\right), \\
d_{\mathrm{m}} & =d_{0}\left(1+\frac{1}{\sqrt{2}} q_{2}-\frac{1}{\sqrt{6}} q_{3}\right) .
\end{aligned}
$$

While a positive $q_{3}$ corresponds to the pattern shown in the previous subsection leading to alternating elongation and compression in the $a b$-plane and compression along $c, q_{2}$ lifts the degeneracy of $d_{\mathrm{m}}$ and $d_{\mathrm{s}}$. As will be seen later, the chosen basis is very generic for $\mathrm{PCMO}$, in which the octahedral distortions can be described mainly by $q_{3}$ with small contributions of $q_{2}$. Since according to Eq. (2.3), (2.4), and (2.6) all lattice parameters are proportional to $d_{0}$, the anisotropy of the Pbnm unit cell in the absence of twisting is fully described by $\left(n_{2}, \theta, q_{2}, q_{3}\right)$. Resulting values for $a, b$, and $c^{\prime}=c / \sqrt{2}$ as a function of the tilt angle are presented in Fig. 2.5 for (top) $q_{2}=q_{3}=0$, (center) $q_{2}=0, q_{3} \neq 0$, and (bottom) $q_{2} \neq 0, q_{3} \neq 0$. In all cases, $n_{2}$ was set to $1 / \sqrt{2}$. In the absence of distortions, all lattice parameters decrease with increasing $\theta$ where $a$ is affected the most since the component $n_{1}$ is generally small (see Fig. 2.4) leading to a tilt axis almost orthogonal to $a$. The central plot, including a finite distortion $q_{3}$, shows firstly that as a consequence $c^{\prime}$ is lowered and $a$ and $b$ are increased for small tilt angles. Secondly, it reveals that the in-plane parameters react qualitatively differently to tilts if $q_{3}$ is finite as $b$ increases resp. decreases slightly for small resp. large $\theta$ and $a$ is ever strongly decreasing. Lastly, including a finite $q_{2}$ as shown at the bottom, the splitting of $b$ and $c^{\prime}$ is suppressed while that of $a$ and $b$ with increasing tilt angle is even slightly enhanced.

\subsubsection{Significance of the Structural Degrees of Freedom in $\mathrm{Pr}_{1-x} \mathrm{Ca}_{x} \mathrm{MnO}_{3}$ (PCMO)}

After having shown that the structure of perovskites with untwisted octahedra in a Pbnm unit cell can be fully described by the tupel $\left(n_{2}, \theta, d_{0}, q_{2}, q_{3}\right)$ - as well as two additional positional parameters of the A-site atoms - the underlying parameters shall be quantified for PCMO in this subsection. For this purpose, the data obtained by refined neutron diffraction from [51] and $[20,52]$ will be used. The former reference includes measurements for $x=0$ 

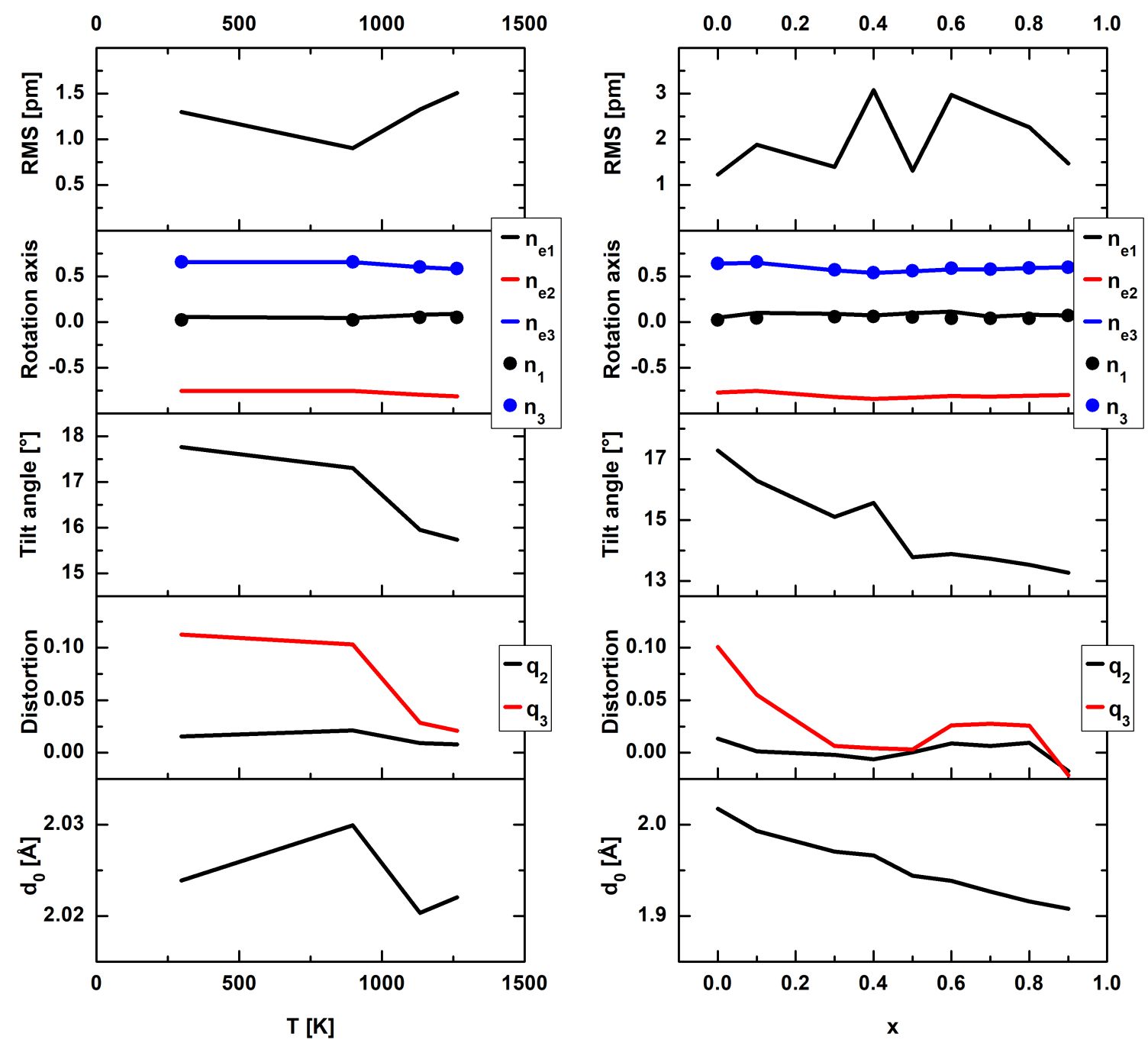

Figure 2.6: Structural parameters extracted from the data given in [51] (left) and $[20,52]$ (right): While $d_{0}, q_{2}$, and $q_{3}$ can be calculated directly, $\theta$ and $\left(n_{\mathrm{e} 1}, n_{\mathrm{e} 2}, n_{\mathrm{e} 3}\right)$ are the solutions of Eq. (2.14) with optimal RMS. In addition, $n_{1}\left(n_{\mathrm{e} 2}, \theta, d_{0}, q_{2}, q_{3}\right)$ and $n_{3}\left(n_{\mathrm{e} 2}, \theta, d_{0}, q_{2}, q_{3}\right)$ as defined in Eq. (2.8) and (2.9) are plotted. 
at elevated temperatures while the latter contain room temperature data in the range $0 \leq x \leq 0.9$. In contrast to $d_{0}, q_{2}$, and $q_{3}$, which can be directly calculated from the given $\mathrm{Mn}$ and $\mathrm{O}$ positions, the extraction of $n_{2}$ and $\theta$ is not trivial since the experimental data weakly violates the assumption of untwisted octahedra. Thus, in order to project the results on the subspace excluding twisting, the experimental rotation axis $\vec{n}_{\mathrm{e}}=\left(n_{\mathrm{e} 1}, n_{\mathrm{e} 2}, n_{\mathrm{e} 3}\right)$ as well as $\theta$ are approximated by solving the optimisation problem:

$$
\min _{\substack{\vec{n}_{\mathrm{e}}, \theta \\\left|\vec{n}_{\mathrm{e}}\right|=1}} \sqrt{\left(\left|\mathbf{R}\left(\vec{n}_{\mathrm{e}}, \theta\right) \vec{r}_{\mathrm{l}}-\vec{r}_{\mathrm{el}}\right|^{2}+\left|\mathbf{R}\left(\vec{n}_{\mathrm{e}}, \theta\right) \vec{r}_{\mathrm{s}}-\vec{r}_{\mathrm{es}}\right|^{2}+\left|\mathbf{R}\left(\vec{n}_{\mathrm{e}}, \theta\right) \vec{r}_{\mathrm{m}}-\vec{r}_{\mathrm{em}}\right|^{2}\right) / 9} .
$$

Here, $\vec{r}_{\mathrm{el}}, \vec{r}_{\mathrm{es}}$, and $\vec{r}_{\mathrm{em}}$ are the experimentally determined positions of the oxygen atoms relative to the central $\mathrm{Mn}$ atom and correspond to the long, short, and medium bonding length. Furthermore, $\vec{r}_{\mathrm{l}}, \vec{r}_{\mathrm{s}}$, and $\vec{r}_{\mathrm{m}}$ denote the oxygen positions of an untwisted octahedron with the same bonding lengths prior to any rotation. The solutions of Eq. (2.14) were determined using the Nelder-Mead algorithm implemented in the scipy.optimize module in python and are presented together with $d_{0}, q_{2}$ and $q_{3}$ in Fig. 2.6 where the data of [51] resp. [20, 52] is shown left resp. right: The first row presents the root mean square difference of fitted and experimental oxygen positions, i.e. the optimal value of Eq. (2.14). The obtained values of a few picometer compare well to the positional precisions given in the primary sources supporting the assumption that twisting is not significant. Furthermore, the second row reveals that the rotation axis hardly changes with $x$ and $T$. To check for model consistency, $n_{1}\left(n_{\mathrm{e} 2}, \theta, d_{0}, q_{2}, q_{3}\right)$ and $n_{3}\left(n_{\mathrm{e} 2}, \theta, d_{0}, q_{2}, q_{3}\right)$ as defined in Eq. (2.8) and (2.9) are plotted additionally agreeing well with the optimal solutions $n_{\mathrm{e} 1}$ and $n_{\mathrm{e} 3}$. In contrast, the remaining four parameters $\left(\theta, d_{0}, q_{2}, q_{3}\right)$ do show characteristic behaviour, i.e. $\theta$ as well as $d_{0}$ decrease with $x$, which matches the expectations of an increasing Goldschmidt factor (approaching unity) as well as a decreasing Mn valence leading to a smaller ionic radius [19]. In addition, while $q_{2}$ is generally small, $q_{3}$ increases for low $x$ and $T$ with exceptional behaviour for $0.5<x \leq 0.8$. Lastly, $\theta, d_{0}$, and $q_{3}$ show a rapid decrease around $T=1000 \mathrm{~K}$ for $x=0$ leading to an orthorhombic to pseudo-cubic phase transition [19,51]. Possible driving forces of this transition and its relation to the correlation phenomena introduced in the next subsection will be discussed later and the main message of this part are the small variations in the rotation axis as well as the generally small values of $q_{2}$. 


\subsubsection{Correlation Phenomena}

As mentioned earlier, TMOPs raised particular interest due to their complex interplay of lattice, orbital, and spin effects leading to a high tunability of physical properties. To understand the underlying correlation phenomena, a brief knowledge about the electronic states is indispensable. Even though the previous two subsections were already specifically designed to describe materials with Pbnm space group and particularly PCMO best, the concepts presented in this subsection are again more general and can also be transferred to other TMOPs.

Classically, in order to understand the electronic configuration on a qualitative level, an ionic picture is used as a starting point [21], i.e. the chemical valence sum $\mathrm{Pr}_{1-x}^{3+} \mathrm{Ca}_{x}^{2+} \mathrm{Mn}_{1-x}^{3+} \mathrm{Mn}_{x}^{4+} \mathrm{O}_{3}^{2-}$ in the case of PCMO. As $\mathrm{Pr}^{3+}$, $\mathrm{Ca}^{2+}$, and $\mathrm{O}^{2-}$ obtain noble gas configuration, the relevant electronic states are given by the Mn $3 d$-orbitals. Compared with the case of an isolated atom shown on the left in Fig. 2.7, in which all five orbitals are degenerate, the presence of the perovskite crystal field leads to an energy splitting. In the ionic picture, this effect can be explained by the fact that the two $e_{\mathrm{g}}$ orbitals point directly to negatively charged oxygen ions resulting in a Coulomb repulsion. Please note that this picture is highly simplifying and hybridisation effects playing a major role in the splitting of energy states will be explained later on.

In accordance with the general Jahn-Teller (JT) theorem [53], the degeneracy of the $e_{\mathrm{g}}$ and $t_{2 \mathrm{~g}}$ levels can be lifted by lowering the symmetry as it is e.g. done by the $q_{3}$ distortion mentioned previously and shown on the right side of Fig. 2.7. Please note that the long axis, being alternatingly located in the $a b$-plane in Fig. 2.2, is drawn vertically here but for the sake of simplicity the resulting subscripts will be used as general representatives of energy levels. Due to the compression in the horizontal directions, the average distance of electrons occupying $d_{x^{2}-y^{2}}$ resp. $d_{x y}$ to neighbouring oxygen ions gets smaller leading again to an increasing energy level. Importantly, the JT effect does not change the mean energy of previously degenerate orbitals hence only partially filled configurations obtain lower total energies. In the case of PCMO, four resp. three $3 d$-orbitals have to be filled for $\mathrm{Mn}^{3+}$ resp. $\mathrm{Mn}^{4+}$. Additionally, due to strong Hund's coupling, each orbital is occupied only once and the spin of electrons align at each lattice site [54]. Please note that in general non-aligned lattice spin states exist in TMOPs as well [55]. Still, in PCMO, $d_{x y}, d_{x z}$, and $d_{y z}$ are occupied in either Mn valence state and $(1-x)$, i.e. the Pr content, determines the number of occupied $e_{\mathrm{g}}$ orbitals. As described thoroughly in the previous subsections, corner-sharing $\mathrm{MnO}_{6}$ 


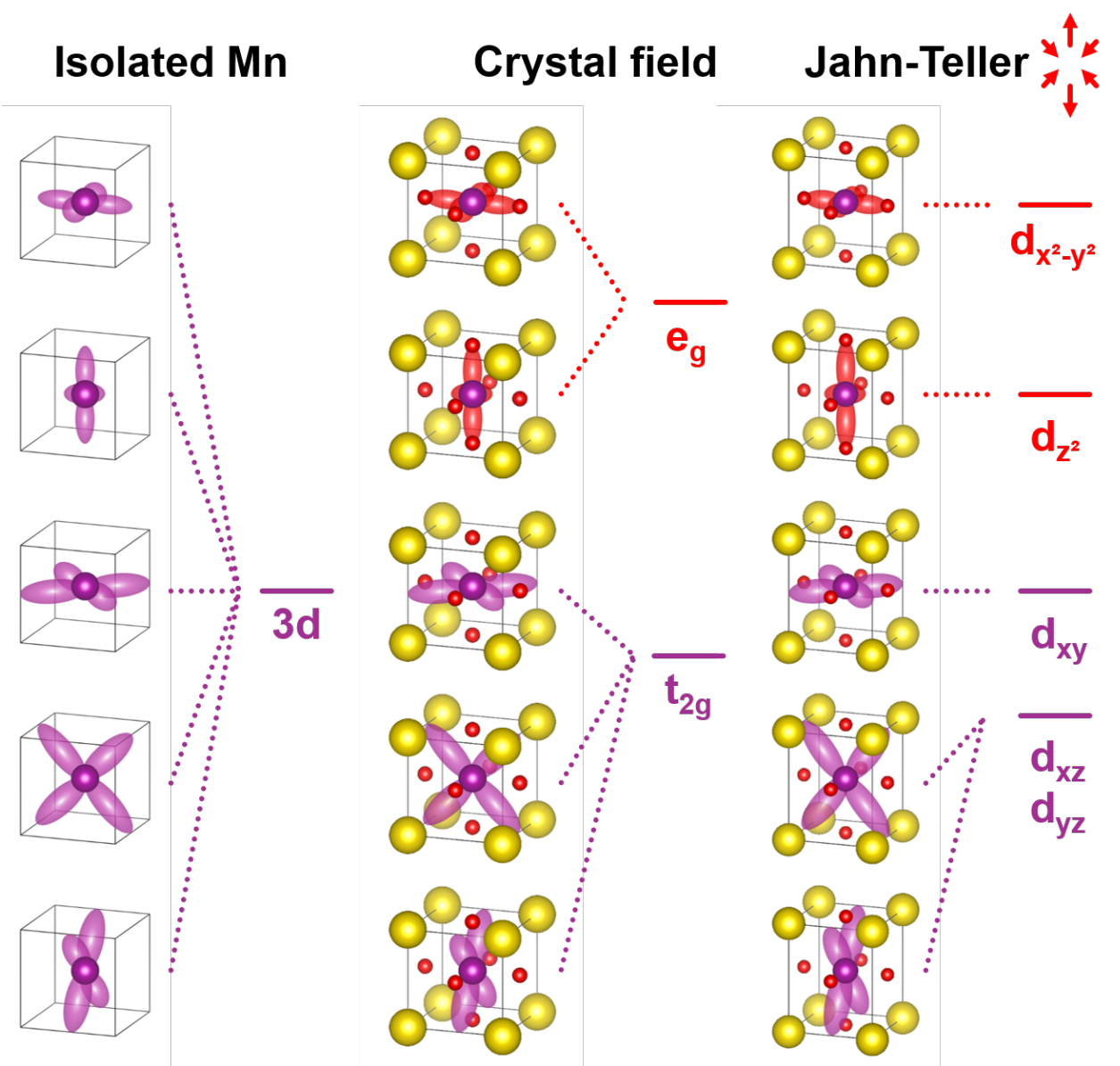

Figure 2.7: Visualisation of the influence of the crystal field and JT effect on the Mn-3d orbitals: The initially degenerate states of the isolated atom (left) are split in two $e_{\mathrm{g}}$ and three $t_{2 \mathrm{~g}}$ levels (middle) due to the crystal field of the perovskite structure. The additional symmetry breaking due to a finite distortion further decreases the degeneracy (right). 
cannot be distorted arbitrarily resulting in a strong coupling between collaborative lattice motions and orbital configurations. Furthermore, the spin system is correlatively linked as well since the orientation of neighbouring lattice spins can enhance electronic exchange processes, e.g. super-exchange in high- and double-exchange in mixed-valence TMOPs [21]. In fact, the latter mechanism first explained the experimentally observed colossal magneto resistance (CMR) effect describing a reduced resistance in the presence of a strong magnetic field aligning the lattice spins [22].

Despite being suitable to explain the appearance of correlation phenomena qualitatively, it is very important to mention that the ionic picture presented above is highly simplifying since in reality a strong hybridisation of $\mathrm{Mn}$ and $\mathrm{O}$ states is present enhancing the energy splitting and leading to non-integer valences [54]: In fact, the depicted $e_{\mathrm{g}}$ levels represent a band formed by anti-bonding states of Mn-3d and O- $2 p$ orbitals. Similarly, nonbonding and bonding states form valence bands represented by the $t_{2 \mathrm{~g}}$ and $\mathrm{O}-2 p$ orbitals in the ionic picture.

\subsubsection{Ordered Phases in PCMO}

As a consequence of the correlation phenomena in PCMO, a variety of ordered phases have been reported involving periodic alignment of magnetic moments, charges, and orbitals: Firstly, at very low temperatures and depending on $x$, anti-ferromagnetic, ferromagnetic and canted structures have been observed experimentally and confirmed by density functional theory (DFT) calculations [20, 56]. Secondly, in the medium calcium doping range, charge ordering (accompanied with orbital ordering) emerges at medium low temperatures resulting in a two-phase regime of coexisting ordered and disordered domains [57]. Thirdly, as suggested by the Goodenough-KanamoriAnderson (GKA) [21] rules and confirmed by DFT calculations [56], at $T=0$ and for $x=0$ (and possibly small finite values) orbital ordering is to be expected. Being of major interest in this thesis, the latter two phenomena will be discussed in detail in this subsection.

Fig. 2.8 shows a sketch of the spin, charge, and orbital configuration in the $a b$-plane for $T=0$ and (a) $x=0.5$ as well as (b) $x=0$ reproduced from [56]. For the sake of simplicity, only Mn sites and figurative $e_{\mathrm{g}}$ orbitals representing hybridised states are drawn. Spin directions are indicated by black arrows and alternate along the $c$-direction whereas the charge and orbital configurations maintain unchanged. In the charge ordered phase at $x=0.5$, as indicated by the yellow line, ferromagnetic zig-zag chains appear being elongated along $b$. The charge ordering manifests in lower resp. 

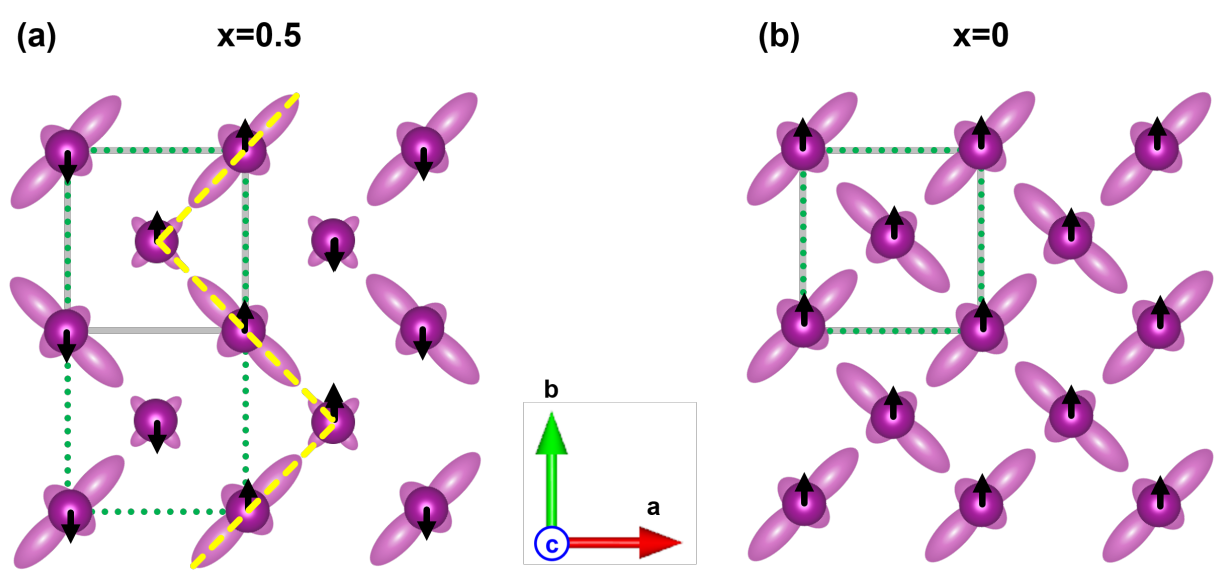

Figure 2.8: Expected spin, charge, and orbital configuration at $T=0$ for (a) charge ordering with $x=0.5$ and (b) orbital ordering with $x=0$. Black arrows represent spin directions and the grey resp. green rectangles indicate the unit cell in the disordered resp. ordered phase excluding spin effects. The orbital's shape and size are explained in the main text and the sketch is reproduced from [56].

higher Mn valences at corner resp. bridging sites of the chains. The charge

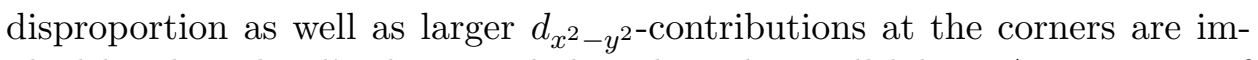
plied by the orbital's shape and the relatively small lobes. As a matter of fact, the Pbnm unit cell obtained in the disordered phase and indicated by the solid grey rectangle exhibits non-equivalent orbitals at the corners and thus does not capture the periodicity of the charge ordered crystal. In fact, limiting the discussion to spin-insensitive diffraction techniques, the dotted green rectangle with equivalent corners (except for the spin) reveals that a $2 b$-superstructure but no change in $a$ and $c$ direction is to be expected, which has been confirmed experimentally by electron diffraction [57]. Furthermore, it was shown that the ordering is accompanied by changes in the atomic positions enhancing the emergence of the superstructure reflections. In contrast, in the orbitally ordered phase for $x=0$ shown in Fig. 2.8 (b), no charge disproportion occurs as one $e_{\mathrm{g}}$ orbital has to be filled per lattice site leaving the crystal symmetry unchanged. The orbital alignment can be well-described using the GKA rules for super-exchange [21]. Furthermore, it is expected that the above mentioned alternating distortion, i.e. $q_{3}$, is enhanced in the orbitally ordered state due to the JT effect as it matches the underlying pattern and lowers the energy of all involved $e_{\mathrm{g}}$ orbitals si- 
multaneously. Nevertheless, a finite $q_{3}$ does not directly prove ordering as it could also be related to steric effects, i.e. the mismatch of ionic radii, leaving the question if the transition coincides with the pseudo-cubic transition discussed earlier and presented in Fig. 2.6 open up till now.

\subsubsection{Influence of Ordering on the Photovoltaic Activity of PCMO thin films on $\mathrm{SrTi}_{1-y} \mathrm{Nb}_{y} \mathrm{O}_{3}$ (STNO)}

Lastly in the context of TMOPs, the photovoltaic (PV) characteristics of ordered PCMO phases will be considered. As will be described in the next section in detail, a material junction is needed to form a rectifying device. For this purpose, $\mathrm{SrTi}_{1-y} \mathrm{Nb}_{y} \mathrm{O}_{3}$ (STNO), i.e. a cubic TMOP [58], has been proven to be a suitable substrate since it firstly enables epitaxial growth of PCMO and secondly exhibits $n$-type behaviour [59] forming a $p$ - $n$ junction with $p$-type PCMO where the latter claim is inferred from a positive Seebeck coefficient [60]. Importantly, due to the strong electron-phonon-coupling described previously, the charge carriers in PCMO and STNO are of polaronic nature [59, 61], i.e. bound states of holes resp. electrons and phonons. More specifically, in the case of PCMO, self-trapped small polarons emerge leading to a thermally activated hopping behaviour of the mobility [61], i.e. a lower mobility for decreasing $T$. Nevertheless, it was found experimentally that the open-circuit voltage as a fingerprint of the PV efficiency increases strongly while undergoing the charge ordering phase transition of PCMO $(x=0.34)$ discussed earlier [17, 62]. Consistently, pump-probe absorption revealed an increased lifetime of hot polarons being in agreement with theoretical calculations modelling the ordered phase by a Hamiltonian including a penalty term for structural deviations from the initial collaborative configuration [18]. Altogether, these findings suggest that collaborative ordering can suppress phononic relaxations of hot polarons and similar behaviour was recently found in the charge ordered phase of Ruddlesdon-Popper PCMO with a significantly higher transition temperature [27]. Surprisingly, similar behaviour is found for PCMO $(x=0.1)$ below room temperature but well above the magnetic transition [28] raising the question if a collaborative phase transition occurs in this temperature regime. In fact, as described in the last subsection, there is no evidence that the orbital ordering coincides with the pseudo-cubic transition leaving it as a possible candidate. 


\subsection{Photovoltaic Effect in Classical Semiconduc- tors}

As the second major part of the theoretical background, this section will focus on $p$ - $n$ junctions in classical semiconductors and relevant equations describing their response to external stimuli like biasing or excess charge carrier generation. Lastly, the role of serial and parallel resistors will be discussed using a one diode equivalent circuit.

\subsubsection{The $p$ - $n$ Junction}

As already mentioned in the context of perovskite-based photovoltaic junctions, rectifying devices can for example be formed by a stack of $n$ - resp. $p$-type material and the resulting $p$ - $n$ junction is the natural candidate to introduce relevant concepts describing the physical properties of diodes. Nevertheless, it is worth mentioning that rectifying behaviour in general can be caused by different junctions as well, e.g. by including an intermediate intrinsic region [63] or forming metal-semiconductor interfaces [64]. Bringing $p$ - and $n$-type material in contact, driven by the chemical potential, the respective majority charge carriers on each side diffuse into the adjacent material where they are considered as minority charge carriers and thus likely find a partner to recombine with. Consequently, as this diffusion process causes a net current of free charge carriers, the remaining immobile ionised dopants are no longer fully screened leading to an electric field at the interface counteracting the flow of charged quasi-particles and establishing electro-chemical equilibrium [65]. The underlying drift-diffusion problem is described in a semi-classical picture by the following set of differential equations for the electrostatic potential $\phi$ as well as the electron resp. hole densities $n$ resp. $p$ [65]:

$$
\begin{array}{r}
-\vec{\nabla}\left(\epsilon_{0} \epsilon_{\mathrm{r}} \vec{\nabla} \phi\right)=e\left(C_{\mathrm{D}}-C_{\mathrm{A}}-n+p\right), \\
0=\frac{\mathrm{d} n}{\mathrm{~d} t}=-\vec{\nabla}\left(\mu_{\mathrm{e}} n \vec{\nabla} \phi-D_{\mathrm{e}} \vec{\nabla} n\right)+g-r, \\
0=\frac{\mathrm{d} p}{\mathrm{~d} t}=-\vec{\nabla}\left(-\mu_{\mathrm{h}} p \vec{\nabla} \phi-D_{\mathrm{h}} \vec{\nabla} p\right)+g-r .
\end{array}
$$

Other than the dielectric constant $\epsilon_{0}$, the elementary charge $e$, and the Boltzmann constant $k_{\mathrm{B}}$, the permittivity $\epsilon_{r}$, the electron resp. hole mobility $\mu_{\mathrm{e}}$ resp. $\mu_{\mathrm{H}}$ as well as diffusion coefficient $D_{\mathrm{e}}$ resp. $D_{\mathrm{h}}$, and the concentration of ionised donors $C_{\mathrm{D}}$ resp. acceptors $C_{\mathrm{A}}$ are spatially dependent and can be 
described by step functions in the case of an abrupt junction. Furthermore, $g$ and $r$ denote the excess charge carrier generation and recombination rates and as the term excess implies $g \equiv r \equiv 0$ holds in the equilibrium state. Please note that setting the right part in Eq. (2.16) and (2.17) to zero describes the stationary case.

In order to approximate solutions of Eq. (2.15)-(2.17), the space charge region (SCR), i.e. the domain with non-screened ionised dopants, is often assumed to be box-shaped and fully depleted in free carriers fixing the right side of Eq. (2.15) and thus the electric potential [65]. In a second step, Eq. (2.16) and (2.17) can be solved for a fixed $\phi$ where the exact solution depends on the involved material parameters as well as the model describing the statistics of charge carriers, i.e. on whether e.g. effective mass or Boltzmann approximations are used or not [65]. Conceptually, this so-called box approximation is somewhat inconsistent as $n$ and $p$ are assumed to be discontinuous at the edges of the SCR to solve Eq. (2.15) but $\phi$ remains continuous inheriting this property to $n$ and $p$ in Eq. (2.16) and (2.17). Nevertheless, due to the rapid change of free charge carrier concentrations caused by small changes in $\phi$, e.g. exponential for non-degenerate semiconductors [65], the resulting inconsistencies tend to be small. The diffusion voltage $V_{\mathrm{D}}$ counteracting the chemical gradient enters as a fixed boundary condition, which determines the extension of the depletion zone and can be easily adopted to the situation of an externally applied voltage $U$ by substituting $V_{\mathrm{D}}$ with $\left(V_{\mathrm{D}}-U\right)$. Consequently, using the box approximation, the SCR width can be calculated as a function of $U$ and is given for an abrupt junction by [66]:

$$
d_{\mathrm{SCR}}=\left(1+\frac{N_{\mathrm{D}}}{N_{\mathrm{A}}}\right) \sqrt{\frac{2 N_{\mathrm{A}} \epsilon_{0} \epsilon_{n} \epsilon_{p}\left(V_{\mathrm{D}}-U\right)}{e N_{\mathrm{D}}\left(\epsilon_{n} N_{\mathrm{D}}+\epsilon_{p} N_{\mathrm{A}}\right)}} .
$$

Here, $N_{\mathrm{D}}$ resp. $N_{\mathrm{A}}$ denote the concentration of ionised donors resp. acceptors in the $n$ - resp. $p$-type material. Please note that the previously mentioned inconsistencies are expected to gain in significance when the charge carrier injection into the space charge region is high, i.e. when the current caused by $U$ is large, contradicting the assumption of deep depletion.

In order to derive the current-voltage characteristics of a $p$ - $n$ junction, more care about underlying assumptions has to be taken: Firstly, in accordance with the box approximation, weak injection shall be supposed. Furthermore, by assuming that the entire voltage drop occurs within the SCR, i.e. a negligible resistivity of the neutral semiconductors, a homojunction is present, i.e. $n$ - and $p$-material differ only in doping concentrations, and 
Boltzmann statistics apply, the following equation can be deduced [66, 67]:

$$
I(U)=I_{\mathrm{s}}\left(\exp \left(\frac{e U}{n k_{\mathrm{B}} T}\right)-1\right) .
$$

Here, $n$ is the ideality factor of the diode, which depends on the charge transport mechanism across the interface and will be discussed further in the following subsection. In [66], the validity of (2.19) (with an adapted definition of $I_{\mathrm{S}}$ ) was extended to the case of heterojunctions if the voltage drops solely in one of the involved materials, i.e. if:

$$
\frac{\epsilon_{n} N_{\mathrm{D}}}{\epsilon_{p} N_{\mathrm{A}}} \approx 0 \quad \text { or } \quad \frac{\epsilon_{p} N_{\mathrm{A}}}{\epsilon_{n} N_{\mathrm{D}}} \approx 0 .
$$

\subsubsection{Excess Charge Carrier Generation and Recombination}

The phenomena of excess charge carrier generation and recombination were already mentioned above as they enter in Eq. (2.16) and (2.17) and will be discussed further in this subsection. Since Eq. (2.15) is solved independently of the latter in the box approximation, $r$ and $g$ have no impact on Eq. (2.18). On the contrary, the current-voltage characteristic does depend on the involved recombination process and is accounted for by the introduction of the ideality factor $n$ [68]. Typically, three qualitatively different bulk recombination processes are considered [65]: (a) Radiative recombination causing the emission of a photon with energy equal to the energy difference of the annihilated states. (b) Auger recombination involving another free carrier which is lifted in energy instead of a photon. (c) Shockley-ReadHall (SRH) recombination describing annihilation mediated by trap states. In the latter case, the energy conservation is in contrast to the former not obvious but realised e.g. by cascades including the creation of phonons or photons [69]. The corresponding excess charge carrier recombination rates are given (in the same order and for a single SRH trap state) by [65]:

$$
\begin{aligned}
r_{\mathrm{rad}} & =r_{\mathrm{eh}}\left(n p-n_{i}^{2}\right), \\
r_{\mathrm{Aug}} & =\left(r_{\mathrm{eeh}} n+r_{\mathrm{ehh}} p\right)\left(n p-n_{i}^{2}\right), \\
r_{\mathrm{SRH}} & =\frac{1}{r_{\mathrm{ht}}^{-1}\left(n-n_{\mathrm{t}}\right)+r_{\mathrm{et}}^{-1}\left(p-p_{\mathrm{t}}\right)}\left(n p-n_{i}^{2}\right) .
\end{aligned}
$$

Here, $n_{i}$ is the intrinsic charge carrier concentration, $r_{\mathrm{eh}}$ the radiative recombination rate, $r_{\text {eeh }}$ resp. $r_{\text {ehh }}$ the Auger recombination rate per unit concentration of electrons resp. holes, and $r_{\text {et }}$ resp. $r_{\text {ht }}$ the SRH capture 
rates for electrons resp. holes. Furthermore, $n_{\mathrm{t}}$ and $p_{\mathrm{t}}$ denote the electron resp. hole concentrations which would be obtained if the Fermi energy coincided with the trap level. In fact, it can be shown that in the extreme cases of dominating radiative resp. SRH recombination the ideality factor amounts to $n=1$ resp. $n=2$ [68].

In addition to bulk recombination, the broken symmetry at the surface of a finite semiconductor can lead to localised states enhancing the recombination rate locally [65]. Mathematically, this can be accounted for by Neumann boundary conditions of the set of differential equations given in Eq. (2.15)-(2.17). Even though the corresponding surface recombination term technically obtains a similar form like $r_{\mathrm{SRH}}$ in Eq. (2.23), it is often introduced in the limit of low injection and a Fermi level well above resp. below the trap state in $n$-type resp. $p$-type semiconductors [65]:

$$
\begin{aligned}
& \vec{\nabla} n \cdot \hat{s}=\vec{\nabla} p \cdot \hat{s}=\frac{S_{\mathrm{h}}}{D_{\mathrm{h}}}\left(p-p_{0}\right), \text { for } n \text {-type } \\
& \vec{\nabla} n \cdot \hat{s}=\vec{\nabla} p \cdot \hat{s}=\frac{S_{\mathrm{e}}}{D_{\mathrm{e}}}\left(n-n_{0}\right), \text { for } p \text {-type } .
\end{aligned}
$$

$S_{\mathrm{h}}$ resp. $S_{\mathrm{e}}$ are the corresponding surface recombination velocities and are - as the subscripts suggest - properties of the minority charge carriers. Furthermore, $\hat{s}$ denotes the surface normal vector and $p_{0}$ resp. $n_{0}$ the minority charge carrier concentrations in the $n$ - resp. $p$-type bulk material in equilibrium. Obviously, as the role of electrons and holes swap while crossing the SCR of a $p-n$ junction, it is to be expected that Eq. (2.24) and (2.25) are only valid in the neutral semiconductor regions. Furthermore, it is important to mention that charging due to the occupation of surface states is not captured by the recombination terms but demands for modifications in Eq. (2.15).

Lastly, consequences of finite excess charge carrier generations shall be discussed. Supposing a point-like generation rate and keeping the previously used assumption of low injection in a field-free semiconductor with a well-defined majority charge carriers species, it can be shown that the local increase of $n$ and $p$ due to the generation term decays exponentially in space [65]. The corresponding decay length is referred to as the excess charge carrier diffusion length $L$ and depends on the recombination parameters of Eq. (2.21)-(2.23) facilitating the transition back to the equilibrium state. Consequently, in the context of $p$ - $n$ junctions and their photovoltaic activity, $L$ determines the length scale on which generated excess charge carriers can diffuse to the SCR where electrons and holes drift in opposed direction due to the internal field resulting in a photocurrent [70]. Thus, 
the efficiency of a solar cell tends to be high if the light is absorbed, i.e. excess carriers are generated, within a distance from the SCR of a few $L$ (but generally other properties, e.g. surface recombination, have an impact as well) [71]. In the easiest case, the excess charge carrier separation leads to a voltage-independent negative shift of the current in Eq. (2.19), which, however, depends on the illumination and junction details and is referred to as short-circuit current $I_{\mathrm{sc}}$. Nevertheless, systems with voltage-dependent differences between the illuminated and non-illuminated state exist in general as well [68].

\subsubsection{Photovoltaic Effect in the One Diode Model}

As explained in the last subsection, the generation of excess charge carriers in the vicinity of the SCR of a $p$ - $n$ diode leads to a constant negative shift in Eq. (2.19), which is referred to as the short-circuit current $I_{\mathrm{sc}}$ under the given illumination conditions. In order to elaborate under which conditions $I_{\mathrm{sc}}$ can be measured experimentally if previously omitted serial and parallel resistances $R_{\mathrm{s}}$ and $R_{\mathrm{p}}$ are present, the one diode model as it has been presented e.g. in [70] shall be used. Fig. $\quad 2.9$ illustrates the underlying equivalent circuit including the voltage-independent $I_{\mathrm{sc}}$ (disentangled from the diode itself), $R_{\mathrm{S}}$, and

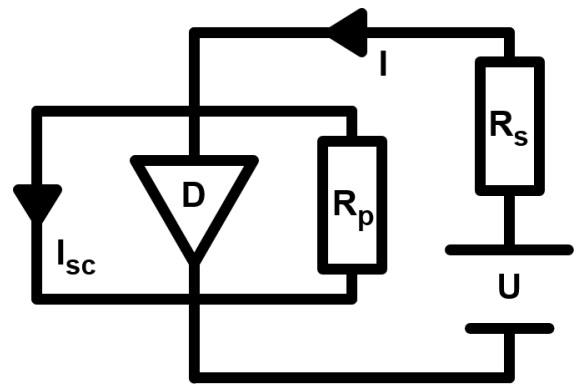

Figure 2.9: Equivalent circuit of the one diode model including a voltageindependent short-circuit current $I_{\mathrm{sc}}$, a serial and parallel resistance $R_{\mathrm{S}}$ and $R_{\mathrm{p}}$ as well as an externally applied voltage $U$ in addition to the diode $D$ described by Eq. (2.19).

$R_{\mathrm{p}}$ as well as an externally applied voltage $U$ and the diode in question following the current-voltage characteristic of Eq. (2.19). Using Kirchhoff's first law leads to the following condition:

$$
0=I\left(1+\frac{R_{\mathrm{s}}}{R_{\mathrm{p}}}\right)-I_{\mathrm{sc}}-\frac{U}{R_{\mathrm{p}}}-I_{\mathrm{s}}\left(\exp \left(\frac{e\left(U-R_{\mathrm{s}} I\right)}{n k_{\mathrm{B}} T}\right)-1\right) .
$$

In addition, the diode resistance shall be defined as

$$
R_{\mathrm{d}}=\frac{n k_{\mathrm{B}} T}{e I_{\mathrm{S}}}
$$


serving as a suitable unit to express involved resistances. Since Eq. (2.26) cannot be solved analytically, the implicitly defined values $I\left(I_{\mathrm{sc}}, U, R_{\mathrm{s}}, R_{\mathrm{p}}\right)$ will be extracted using Newton's method in the following. Fig. 2.10 shows exemplary $I-V$ curves for $I_{\mathrm{sc}}=0$ and $n=1$ : Going from the purely exponential extreme case $R_{\mathrm{s}}=0, R_{\mathrm{p}}=\infty$ (black) to a finite parallel resistance leads to an overall increase in the absolute value of underlying currents and especially to much larger reverse currents (red). Contrarily, by including a finite serial resistance, the absolute value of $I$ is decreased and the strong forward currents are suppressed.

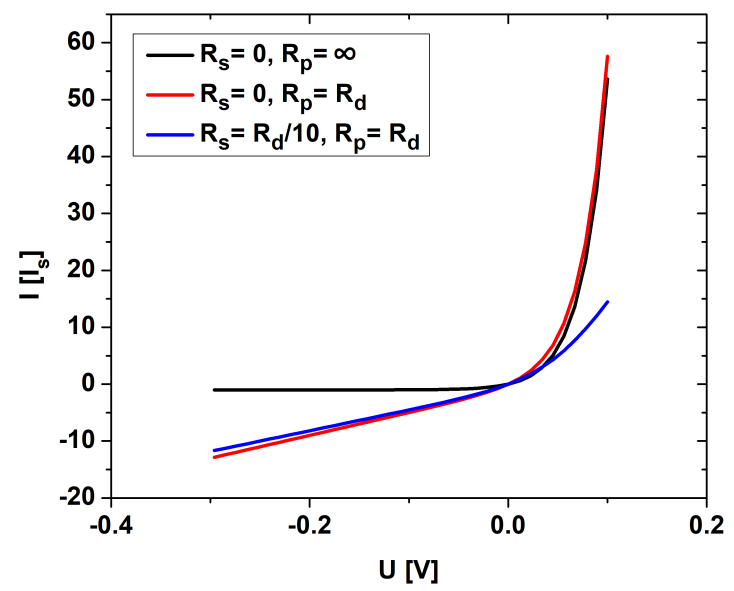

Figure 2.10: $I-V$ curves for an ideal diode (black), in combination with a finite parallel resistance (red) as well as both a serial and parallel resistance (blue).

In this work, the experimental observation of $I_{\mathrm{sc}}$ is of major interest, which is why it is important to analyze the equivalent circuit's behaviour for finite values of the latter as well as to mention that conceptually the idealised case $R_{\mathrm{S}}=0$, i.e. a vanishing input resistance of the amperemeter, cannot be achieved. In order to assess potential influences of $R_{\mathrm{S}}$ and $R_{\mathrm{p}}$ on the experimentally measurable current $I$, the color-coded ratio $I / I_{\mathrm{sc}}\left(U=0, R_{\mathrm{s}}, R_{\mathrm{p}}\right)$ is shown in Fig. 2.11 resp. 2.12 for $I_{\mathrm{s}}=-I_{\mathrm{sc}}=1 \mathrm{~mA}$ resp. $I_{\mathrm{s}}=-I_{\mathrm{sc}} / 10=1 \mathrm{~mA}$. The dashed line indicates the contour line where the ratio equals to 0.9 . As a matter of fact, two qualitatively different phenomena leading to decreased ratios can be observed: In the lower left corners, i.e. where the dashed line is diagonal, solely the ratio of $R_{\mathrm{s}}$ and $R_{\mathrm{p}}$ 


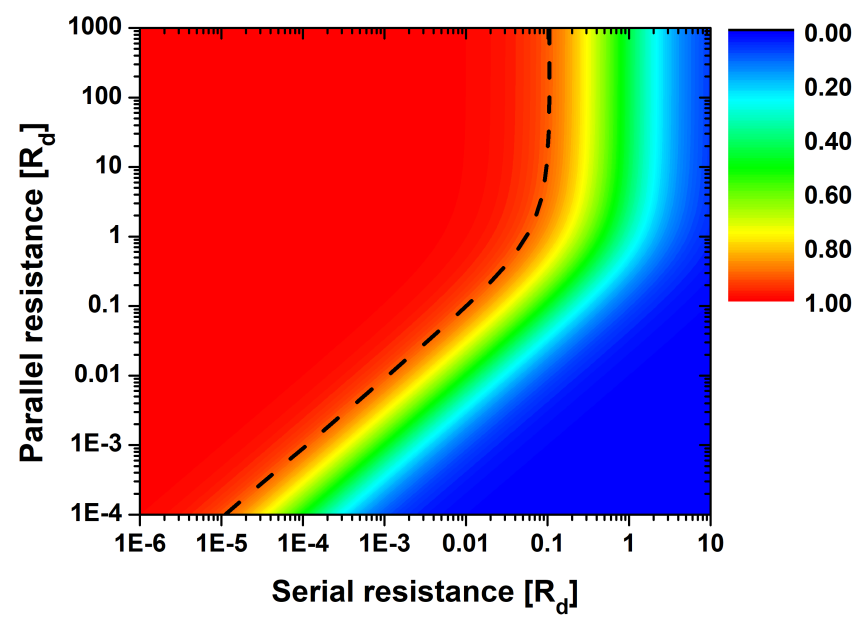

Figure 2.11: Colour-coded fraction $I / I_{\mathrm{sc}}$ in the one diode model obtained from Eq. (2.26) for $I_{\mathrm{s}}=-I_{\mathrm{sc}}=1 \mathrm{~mA}$ as a function of $R_{\mathrm{s}}$ and $R_{\mathrm{p}}$. The dashed line indicates the contour line of 0.9 .

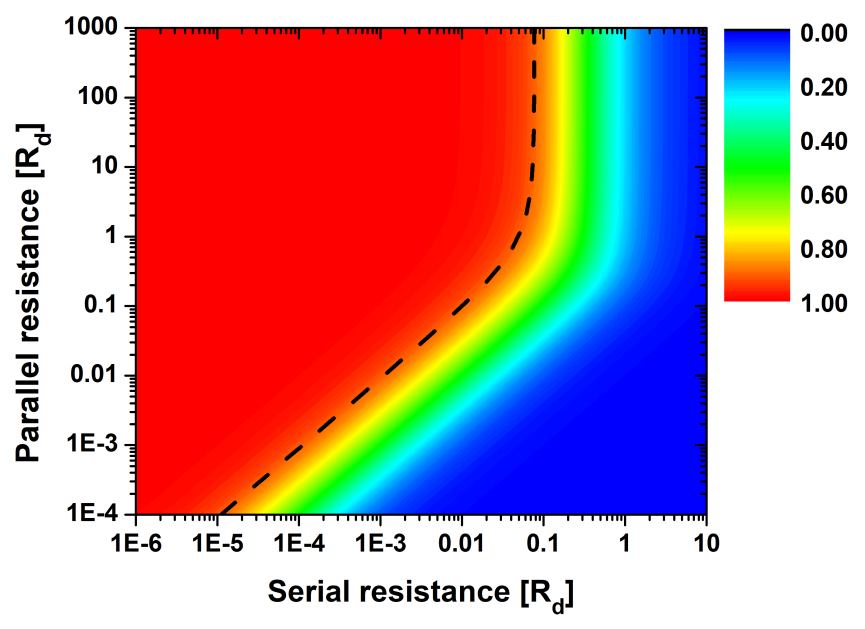

Figure 2.12: Colour-coded fraction $I / I_{\mathrm{sc}}$ in the one diode model obtained from Eq. (2.26) for $I_{\mathrm{s}}=-I_{\mathrm{sc}} / 10=1 \mathrm{~mA}$ as a function of $R_{\mathrm{s}}$ and $R_{\mathrm{p}}$. The dashed line indicates the contour line of 0.9 . 
matters as comparable values lead to a splitting of $I_{\mathrm{sc}}$ to the corresponding resistor branches in Fig. 2.9. In the upper right corners, i.e. where the dashed line is vertical, small changes of $R_{\mathrm{p}}$ are irrelevant, but as the comparison of the two plots shows, $R_{\mathrm{S}}$ as well as the ratio of $I_{\mathrm{sc}}$ and $I_{\mathrm{s}}$ matter. This qualitative change appears as $R_{\mathrm{s}}$ approaches $R_{\mathrm{d}}$ and currents flowing through the former lead to voltage drops across the diode high enough to cause significantly enhanced diffusion currents. Here, $I_{\mathrm{sc}} / I_{\mathrm{s}}$ becomes relevant since the mentioned voltage drop is proportional to the product $I \cdot R_{\mathrm{s}}$ and not purely given by the resistance itself.

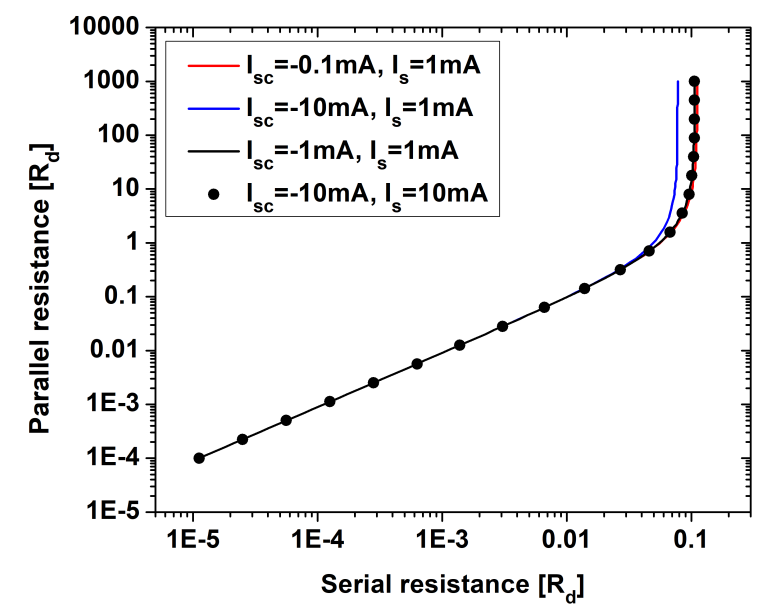

Figure 2.13: Contour line $I / I_{\mathrm{sc}}=0.9$ obtained from Eq. (2.26) as a function of $R_{\mathrm{s}}$ and $R_{\mathrm{p}}$ for different choices of $I_{\mathrm{sc}}$ and $I_{\mathrm{s}}$.

In order to present the injection dependence more clearly, the contour lines $I / I_{\mathrm{sc}}\left(U=0, R_{\mathrm{s}}, R_{\mathrm{p}}\right)=0.9$ for different choices of $I_{\mathrm{S}}$ and $I_{\mathrm{sc}}$ are shown in Fig. 2.13. Firstly, the plot confirms that higher injection levels lower the values of the corresponding $R_{\mathrm{S}}$ in the upper right regime. Secondly, by comparing the black points and line with likewise scaled values for $I_{\mathrm{s}}$ and $I_{\mathrm{sc}}$, the invariance of Eq. (2.26) under the transformation $\left(I, I_{\mathrm{s}}, I_{\mathrm{sc}}, R_{\mathrm{s}}, R_{\mathrm{p}}\right) \rightarrow$ $\left(\alpha I, \alpha I_{\mathrm{s}}, \alpha I_{\mathrm{sc}}, R_{\mathrm{s}} / \alpha, R_{\mathrm{p}} / \alpha\right)$ for $\alpha \neq 0$ is demonstrated. Thirdly, as already mentioned, the presented data confirms that in the lower left regime the ratio of $I_{\mathrm{sc}}$ and the experimentally measurable current $I$ is independent of $I_{\mathrm{sc}}$ itself, which can be a valuable information if the latter's qualitative dependence of other parameters shall be observed. 


\section{Chapter 3}

\section{Experimental Methods}

In this chapter, a brief overview of the herein used experimental techniques shall be given. The first two sections present fundamental principles as well as recent developments in scanning electron microscopy (SEM) and transmission electron microscopy (TEM). Obviously, the two fields have a certain overlap and several comparisons related to resolution power as well as versatility in instrumentation will be given. Lastly, the experimental setups used in this thesis will be described.

\subsection{Scanning Electron Microscopy (SEM)}

The first SEM was presented by M. von Ardenne in [72] followed by an ever increasing application in various scientific disciplines [73]. In this section, the general setup of an SEM as well as its combination with focused ion beam (FIB) shall be introduced. Furthermore, a brief overview of SEM-based electron beam induced current (EBIC) as a powerful tool in semiconductor characterisation will be given.

\subsubsection{General Setup of an SEM}

Rudimentarily, the setup of an SEM can be summarised as an electron emitting and accelerating gun, a probe-focusing lens system as well as a scanning system shifting the focused probe across the investigated sample [73]. Synchronously to the scanning movement, signals caused by the interaction of the electron beam with the specimen can be recorded resulting in spatially resolved information. Prominent examples of detectable signals are backscattered primary electrons as well as secondary electrons being emitted due 
to inelastic scattering of the impinging beam [73]. The resolution of SEMbased techniques is determined by the size of the incident probe as well as the relevant interaction volume in the sample. While the former is due to the short wavelength of fast electrons generally small compared to optical microscopy and limited by aberrations of the lens system [73], the latter depends strongly on the signal in question as will be described through the example of EBIC later on. One of the cornerstone developments in SEM was the invention of field-emission guns (FEGs) [74] leading to much smaller incident probes due to their high coherence. In addition, a non-exaustive list of further instrumental advances exists including stability, detector variety and sensitivity, as well as in-situ capabilities [73, 75, 76].

\subsubsection{Focused Ion Beam (FIB)}

The general principle of an FIB is analogous to that of an SEM described above where the major difference is given by the emitting gun as the particle beam is formed by ions instead of electrons. While first realisations involved large experimental setups [77], the size of FIBs has decreased tremendously and nowadays they are commonly included in SEM chambers of so-called dual beam machines [78]. Due to the strong inelastic interaction with matter, the impinging ions have a comparatively high sputtering yield enabling their application in order to mill micro- and nano-structures into the inserted sample. As an important consequence, FIBs became a major tool for extraction of thin lamellas being suitable for investigations in the later discussed TEM [79]. On top of that, the possible applications include e.g. ion implantation as well as unique imaging capabilities due to strong channeling contrast [80].

\subsubsection{Electron Beam Induced Current (EBIC)}

The inelastic interaction of the SEM's electron beam with the sample causes amongst other excitations the direct and indirect generation of electronhole-pairs, i.e. excess charge carriers. As described in chapter 2, if the specimen includes a $p$ - $n$ junction or other rectifying devices, this leads to finite short-circuit currents, which is, in this context, referred to as electron beam induced current (EBIC) [81, 82]. Due to the high focusability of electrons, one might expect that local centers, e.g. crystal defects, influencing the drift-diffusion-problem of Eq. (2.15)-(2.17) can generally be resolved on the nanometer scale by measuring the spatially-dependent current signal. Nevertheless, EBIC is a generic example of techniques whose 
resolution is rather limited by the above mentioned interaction volume than the size of the incident electron probe [83]. The classical approach to circumvent this problem is a reduction of the acceleration voltage leading to a smaller penetration depth of electrons, i.e. a more confined interaction volume. Unfortunately, this is accompanied by a higher significance of surface recombination. Consequently, many studies have been conducted in the past to disentangle bulk and surface effects including analytical modelling of the interaction volume [83, 84], numerical simulation of the experimental conditions in the SEM [85-87], as well as the introduction of an effective diffusion length accounting for surface effects in the case of point-like excitations $[29,88,89]$. An adapted model relying on the latter approach will be presented later in the context of scanning transmission EBIC.

\subsection{Transmission Electron Microscopy (TEM)}

The first TEM was demonstrated by M. Knoll and E. Ruska in [90], i.e. earlier than the first SEM, and was operated in the so-called conventional mode, in which a rather broad and collimated illumination is used. Later on, the approach of scanning a focused probe was introduced as well leading to the term of scanning transmission electron microscopy (STEM). In this section, the basic concepts of both operation modes shall be discussed briefly followed by short introductions into selected advanced TEM methods.

\subsubsection{Conventional and High-resolution TEM}

As mentioned above, the term conventional TEM refers to the case of collimated broad illumination of electron transparent samples. Subsequently, after its transmission through the specimen, the electron wave can be detected both in diffraction as well as in real space causing a high versatility of possible information extraction [34]. In addition, by inserting apertures in certain intermediate planes, physical filtering of the wave function in both respective spaces can be performed as well leading to contrasts resembling e.g. lattice orientation or strain [34]. Furthermore, due to the electron's short wave length for typical acceleration voltages of a few hundred kilovolt, picometer resolutions are expected assuming diffraction limitation [91]. Nevertheless, the latter assumption is generally not met as instrumental imperfections drastically lower the information limit, i.e. the highest resolvable spatial frequency [34]. Importantly, the invention of aberration correctors significantly increased the latter in comparison with uncorrected systems [92] and has to be considered as a major breakthrough in high-resolution TEM 
history. It is worth mentioning that phase contrast dominates the resulting image formation and aberration correctors can also be used to enhance the contribution of certain spatial frequencies to the image contrast as it is e.g. done by using a negative spherical aberration [93].

\subsubsection{Scanning Transmission Electron Microscopy (STEM)}

As the name suggests, the main conceptual difference of STEM in comparison with SEM is that the impinging electrons almost exclusively transmit the investigated specimen due to generally higher acceleration voltages and

lower sample thicknesses. Nonetheless, this leads to tremendous differences in resolution and signal accessibility: Firstly, the focusability due to the higher acceleration voltage is better in a STEM which is further facilitated by the employment of probe-forming aberration correctors [94]. Secondly, the interaction volume is much smaller in a STEM as the electrons tend to exit the investigated sample before the beam gets significantly spread [95]. Lastly, as most electrons transmit the specimen and many interact only elastically while doing so, classical SEM signals like secondary electron emission and electron back-scattering are comparatively weak, but new opportunities emerge in forward direction [96]. Here, discriminating between different ranges of the collection angle of scattered electrons leads to the definition of e.g. bright-field (BF), annular dark-field (ADF), or high-angle annular dark-field (HAADF) STEM. Importantly, in contrast to TEM, secondary signals caused by the interaction of the electron beam with the sample can potentially be resolved spatially by STEM as well due to the localised source of primary excitations, i.e. the small interaction volume.

\subsubsection{Electron Energy Loss Spectroscopy (EELS) and En- ergy Filtering}

So far, the inelastic interaction of fast electrons with matter has only been considered as a potential source of electron-hole-pair generation. However, inelastic events serve as a much more versatile information channel as the energy loss of primary electrons corresponds to the energy transfer inside the sample which is needed e.g. for plasmon excitations or lifting electrons from occupied to higher energy unoccupied states [35]. Conceptually, there are two different approaches to extract energy-resolved information: Firstly, by employing a spectrometer, which omits spatial information while dispersing the electron beam, and secondly, by using an image filter keeping this information at the cost of energy resolution [35]. While the former approach 
is usually combined with STEM (maintaining spatial resolution), the latter is typically used in TEM mode, but exceptions exist, e.g. with energyfiltered 4D-STEM as will be discussed later. Generally, the full range of energy losses is divided into three regimes [35]: Firstly, the zero-loss regime including electrons which have interacted solely elastically with the sample and those with energy losses, e.g. due to phonon scattering, that cannot be resolved. Secondly, the low-loss regime including e.g. exciton or plasmon excitations. Thirdly, the core-loss regime including the excitation of tightly-bond core electrons to unoccupied states. As the core levels are element-specific and delocalised final states generally depend on the local environment, information about chemical elements and bonding can be extracted from the core-loss regime as well [35].

Furthermore, by averaging over all possible inelastic events, the materialspecific inelastic mean free path $\lambda$ can be used to determine the thickness $t$ of the investigated sample via [97]:

$$
t=\lambda \log \left(\frac{I_{\text {tot. }}}{I_{\text {ZLP }}}\right) \text {. }
$$

Here, $I_{\text {tot. }}$ is the total electron intensity, i.e. beam current times exposure time, and $I_{\text {ZLP }}$ the intensity of the zero-loss peak.

\subsubsection{Energy-dispersive X-ray Spectroscopy}

Complementary to the primary signal of EELS core-losses discussed in the previous subsection, the induced secondary signal of characteristic X-ray emission as an energy dissipation channel of an excited state can be detected by energy-dispersive X-ray spectroscopy (EDX) [95]. Since the radiative annihilation of core-loss holes can involve any occupied higher-energy state, the sensitivity to local bonding is extremely weak. Hence, EDX is predominantly used to determine chemical compositions [95]. For a fixed chemical composition, the relative intensity of the characteristic lines, which is generally superimposed with bremsstrahlung, depends on the interplay of three phenomena: Firstly, the absorption of X-rays along their escape path from the sample leading to a decreased intensity. Secondly, the fluorescent emission subsequent to absorption of a higher-energy X-ray leading to an increased intensity. Lastly, on the relative product of ionisation cross-sections and Xray yields of the involved elemental lines. In practice, the latter phenomenon is commonly entangled with the detection efficiency of the used system by employing calibrated Cliff-Lorimer factors [98]. In contrast to SEM-based

EDX, absorption and fluorescence effects can often be neglected in the TEM 
due to the small sample thickness leading to the following relation [98]:

$$
\frac{I_{\mathrm{A}}}{I_{\mathrm{B}}}=\frac{c_{\mathrm{A}}}{c_{\mathrm{B}}} \cdot \frac{k_{\mathrm{A}}}{k_{\mathrm{B}}} .
$$

Here, $I_{\mathrm{A}}$ resp. $I_{\mathrm{B}}$ is the detected intensity of characteristic X-rays belonging to a line with Cliff-Lorimer factor $k_{\mathrm{A}}$ resp. $k_{\mathrm{B}}$ for element A resp. B. Furthermore, $c_{\mathrm{A}}$ and $c_{\mathrm{B}}$ denote the concentrations of the respective elements.

\subsubsection{Four-dimensional STEM (4D-STEM)}

4D-STEM has recently gained a lot of popularity and can be conceptually described as taking two-dimensional images (usually diffraction patterns, but occasionally real space images as well) across a two-dimensional scanning array resulting in a four-dimensional dataset. A comprehensive review on 4D-STEM was recently published in [36]. Possible applications include e.g. domain [99], orientation [100], and strain [101] mapping of crystalline materials, strain mapping of amorphous materials [102] as well the extraction of their short- and medium-range order [103], electric [104] and magnetic [105] field mapping, and the creation of STEM signals using virtual detectors [106]. Obviously, due to the high dimensionality, the processing of involved signals demands for numerical treatment of large image stacks hence several projects have been launched to offer publicly available software solutions for efficient 4D-STEM analysis, e.g. LiberTEM [107], py4DSTEM [108], or pycroscopy [109]. During the course of this thesis and contemporaneously with the latter implementations, a 4D-STEM software package has been developed, which will be described in detail in the following chapter including practical examples of data treatment.

\subsubsection{Environmental TEM}

In essence, the short wavelength of fast electrons as well as their strong interaction with matter enable for collection of the above mentioned signals at high spatial resolution. Nevertheless, the latter inherent property also demands for low pressures in the electron optical system to avoid predominant interactions with residual gases. Thus, observing samples under reaction conditions in gaseous or liquid environments is a non-trivial task. Still, facilitated by the advances in instrumental and sample holder design, possibilities in environmental TEM (ETEM) have increased tremendously by pursuing two conceptually different approaches [110]: Firstly, including small differential apertures, the pressure in the sample chamber can be increased locally by letting in a desired gas [111]. Secondly, the specimen can 
be encapsulated in a thin reaction chamber [112]. While the former approach is limited to rather low pressures but as an instrumental modification combinable with most sample holders the latter allows for higher pressures and even liquid environments but causes undesired signals from the encapsulation or comparatively high preparational effort if advanced sidewalls, e.g. graphene [113], are used.

\subsubsection{In-situ Biasing, Heating and Cooling}

The realisation of ETEM setups described in the previous subsection allows for the variation of pressure and chemical potential in the vicinity of the sample (with the mentioned restrictions). In order to control the electric potential as well as the temperature as thermodynamically analogous variables, a variety of dedicated TEM holders have been developed: For the sake of in-situ biasing, holders including mounting and contacting possibilities for piezo-controlled nanotips [114] as well as micro electromechanical systems (MEMS) chips [115] are available. Furthermore, by resistively heating a platinum spiral either at the sample holder's tip [116] or on a MEMS chips carrying the sample [115], TEM experiments can be conducted at elevated temperatures. Here, particularly the latter approach involves only small heating volumes suppressing the effect of thermal drift strongly. In contrast, in-situ cooling is typically realised by cryogenic reservoirs attached to the holder outside the TEM chamber [116] leading to the necessity to cool down larger volumes and thus rather strong thermal drift.

\subsubsection{STEM-EBIC}

The general concept of EBIC, its limited spatial resolution in the SEM due to beam broadening as well as the effect of enhanced excess charge carrier recombination at surfaces have already been described in the previous section. Facilitated by the existence of the in-situ biasing holders mentioned above, EBIC can also be conducted in the STEM (STEM-EBIC) leading on the one hand side to a much more confined interaction volume as the electron beam is weakly broadened before exiting the sample. On the other hand, the exit surface potentially enhances charge carrier recombination as well, and importantly, the control of the surface quality during TEM preparation is extremely limited. Consequently, the surface regions are commonly divided in an amorphised as well as an additional electrically inactive layer [117], in combination referred to as dead layer. The emergence of the latter is typically explained by Fermi level pinning $[118,119]$ but its exact nature and 
whether they are identical in the cited context of off-axis electron holography as well es EBIC remains unclear and might depend on the sample material. However, considering only the region between the dead layers as electrically active and assuming an uncharged interface [120] between bulk-like and dead material, the following thickness-dependent effective diffusion length can be derived for a field-free semiconducting lamella [29]:

$$
L_{\mathrm{eff}}=L \sqrt{1-\frac{2 L}{\left(t-2 t_{\mathrm{d}}\right)\left(\frac{D}{S L}+\operatorname{coth}\left(\frac{t-2 t_{\mathrm{d}}}{2 L}\right)\right)}} .
$$

Here, $D, S$, and $L$ denote the minority carrier diffusivity and surface (more precisely bulk-dead-layer-interface) recombination velocity as well as the bulk diffusion length. In fact, by employing finite element (FE) simulations, in which the dead layers are simply omitted, Eq. (3.3) can be confirmed with remarkable consistence [121].

Even though STEM-EBIC is employed to investigate the electrical response of rectifying junctions to electron-hole-pair generation in this work, it is important to mention that the acronym is used in the context of experimentally similar but conceptually different approaches as well, i.e. in the case of electron beam induced conductivity [122] as well as currents emerging due to secondary electron emission [123, 124].

\subsection{Used Experimental Setups}

After having discussed relevant techniques in electron microscopy briefly, the herein used instrumentation shall be itemised:

- SEM/FIB: FEI Nova 600 NanoLab resp. FEI Helios G4 dual beam

- (E)TEM/STEM: Philips CM200 UT resp. FEI Titan ST ETEM G2 80-300 (image corrected)

- EELS: Gatan Quantum 965 ER

- EDX: Oxford Instruments X-Max $80 \mathrm{~mm}^{2}$

- In-situ biasing and heating: DENSsolutions Lightning D7+ holder

- In-situ cooling: Gatan 613 cooling holder

- Current amplification for EBIC: Stanford Research Systems SR570 


\section{Chapter 4}

\section{Method Development}

A significant part of this thesis consists of TEM method development and will be summarised in this chapter. In more detail, progress in sample preparation techniques, the use of structured apertures, acquisition and analysis of 4D-STEM signals, as well as STEM signal synchronisation will be presented.

\subsection{TEM Sample Preparation}

Despite all advances in TEM instrumentation, a bare necessity to achieve good experimental results remains the preparation of high-quality samples. In fact, as the sensitivity of detectors and the versatility of techniques is ever increasing, demands to sample preparation even grow accordingly. In this section, an innovative planview preparation method as well as strategies for in-situ heating and biasing preparation on MEMS chips will be described.

\subsubsection{Planview Preparation}

In the context of this dissertation, the following paper about planview lamella extraction was created and published in the following manuscript [125] in Ultramicroscopy (doi.org/10.1016/j.ultramic.2021.113320) - the author's contributions are given in the main text. All contents are reprinted in their original form with permission.

By combining mechanical grinding with backside lift-out in the FIB, the benefits of existing sample preparation techniques were combined enabling the extraction of site-specific features with a large field of view without exposing the front surface to the ion beam. A detailed comparison with previous strategies is given in the conclusion. 


\title{
Site-specific plan-view TEM lamella preparation of pristine surfaces with a large field of view
}

\author{
Tobias Meyer $^{\mathrm{a}, 1}$, Tobias Westphal ${ }^{\mathrm{a}, 1}$, Birte Kressdorf $^{\mathrm{b}}$, Ulrich Ross ${ }^{\mathrm{b}}$, Christian Jooss ${ }^{\mathrm{b}}$, \\ Michael Seibt ${ }^{\mathrm{a}, *}$ \\ a 4th Institute of Physics - Solids and Nanostructures, University of Goettingen, Friedrich-Hund-Platz 1, 37077 Göttingen, Germany \\ ${ }^{\mathrm{b}}$ Institute of Materials Physics, University of Goettingen, Friedrich-Hund-Platz 1, 37077 Göttingen, Germany
}

\section{A R T I C L E I N F O}

\section{Keywords:}

TEM

Lamella

Preparation

Plan-view

Site-specific

Field of view

\begin{abstract}
A B S T R A C T
Transmission electron microscopy has become a major characterization tool with an ever increasing variety of methods being applied in a wide range of scientific fields. However, the probably most famous pitfall in related workflows is the preparation of high-quality electron-transparent lamellae enabling for extraction of valuable information. Particularly in the field of solid state physics and materials science, it often required to study the surface of a macroscopic specimen with plan-view orientation. Nevertheless, despite tremendous advances in instrumentation, i.e. focused ion beam, the yield of existing plan-view lamellae preparation techniques is relatively low compared to cross-sectional extraction methods. Furthermore, techniques relying on mechanical treatments, i.e. conventional preparation, compromise site-specifity. In this paper, we demonstrate that by combining a mechanical grinding step prior to backside lift-out in the focused ion beam planview lamellae preparation becomes increasingly easy. The suggested strategy combines site-specifity with micrometer precision as well as possible investigation of pristine surfaces with a field of view of several hundred square micrometers.
\end{abstract}

\section{Introduction}

While the preparation of high-quality site-specific cross-sectional transmission electron microscopy (TEM) lamellae has become rather straight forward due to the developments in focused ion beam (FIB) instrumentation, the extraction of plan-view lamellae matching the same criteria remains comparatively difficult. Consequently, many different approaches employing mechanical and FIB treatments or a combination of both to achieve high-quality plan-view TEM foils have been reported [1]. To the best of our knowledge, these approaches can generally be categorized into two groups: (i) A mechanical treatment e.g. embedding into resin [2], sandwich gluing [3], tripod respectively planar polishing $[4,5]$ - optionally followed by FIB slicing at the edge of the residual bulky sample. (ii) A direct extraction of a block of material from the top using the FIB [6]. Whereas category (i) clearly compromises site-specifity since lamellae can only be extracted at a mechanically prepared edge, the main drawback of category (ii) is the rather complicated and time-consuming excavation as well as the necessity to protect the surface both from ion beam irradiation as well as contamination due to redeposition. Possible solutions are the use of a protective block which is mounted with a micromanipulator [7] or a shielding platinum wall [8]. While in the latter case, residual surface contaminants ultimately had to be removed by FIB milling leading to an altered surface, the authors of [7] managed to remove exclusively the protective block during the thinning process revealing the pristine surface.

In this paper, we demonstrate a novel strategy of combining mechanical polishing and FIB lift-out from the backside to prepare planview TEM lamellae of pristine surfaces as well. Compared to those in category (i), the presented method offers uncompromised site-specifity. In addition, the approach avoids the experimental difficulties of cutting a bulky block from the sample's top as it is done in category (ii) methods enabling for TEM lamellae preparation with a large field of view.

\section{Instrumentation}

Lamellae extraction and thinning has been performed in an FEI Helios G4 Dual Beam FIB. TEM investigations have been conducted in an image-corrected FEI Titan 80-300 operated at $300 \mathrm{kV}$ and equipped with a Gatan Quantum 965 ER image filter for electron energy loss

\footnotetext{
* Corresponding author.

E-mail address: michael.seibt@uni-goettingen.de (M. Seibt).

1 Contributed equally to this work.
} 
a

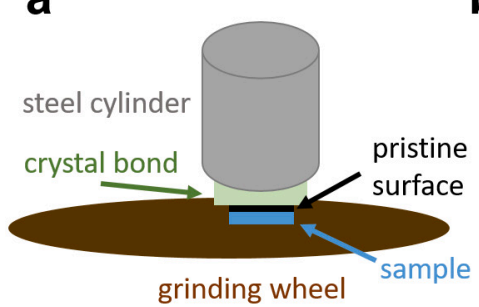

d

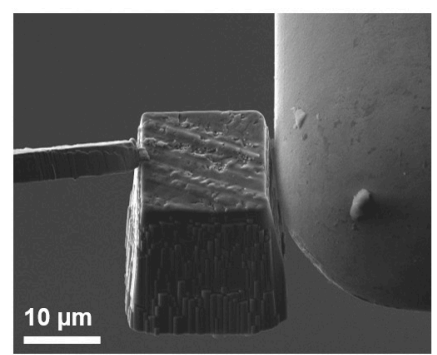

b

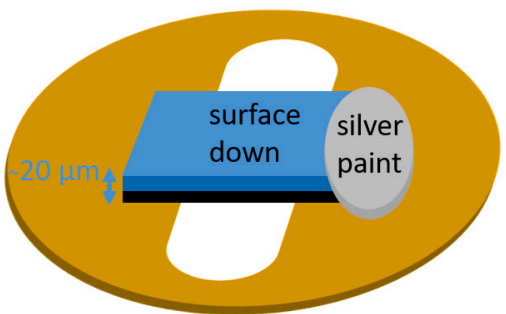

e

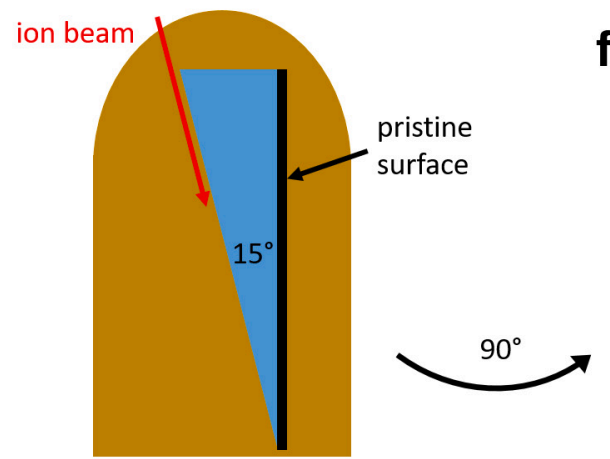

C

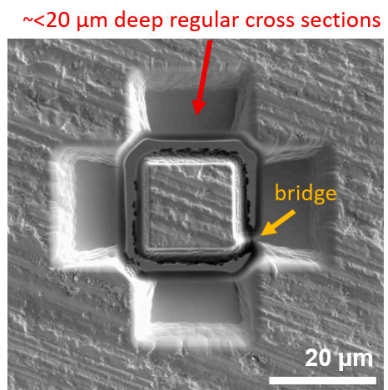

f

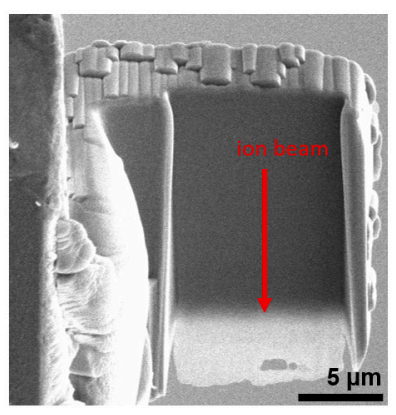

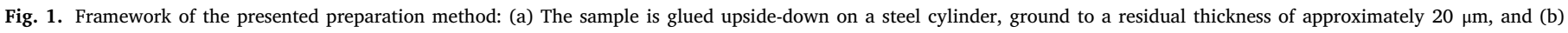
mounted on a slotted copper grid after acetone bathing. (c) A block is lifted out from the backside, i.e., the ground side, using approximately $20 \mu \mathrm{m}$ deep regular cross sections

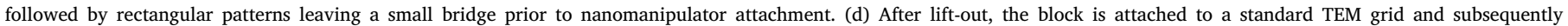

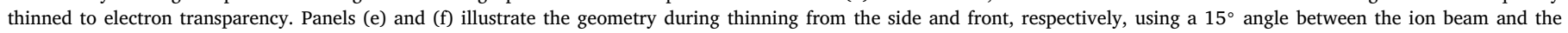
pristine surface. The tilt angle might be varied for final low-kV thinning steps or in order to achieve larger electron-transparent areas as explained in the main text.

Nanomanipulator - Specimen (pristine surface down) $\quad-$ TEM grid $\downarrow$ Ion beam

Pretilted holder

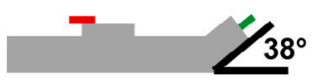

$R=0^{\circ}, T=0^{\circ}$
1: Trench milling

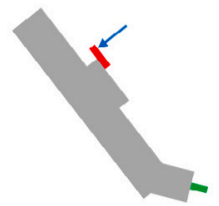

$\mathbf{R}=0^{\circ}, \mathbf{T}=\mathbf{5 2}^{\circ}$
2: Lift-out

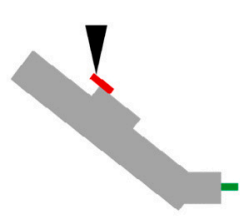

$\mathbf{R}=0^{\circ}, \mathbf{T}=38^{\circ}$
3: Mounting

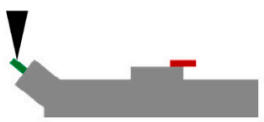

$R=180^{\circ}, T=0^{\circ}$
4: Thinning

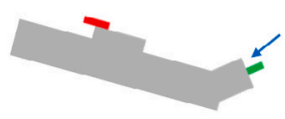

$R=0^{\circ}, T=15^{\circ}$

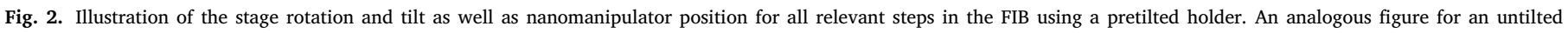
holder design as well as a table including the dimensions and ion beam parameters for all used patterns is provided in Fig. S1 resp. Tab. S1 in the Supplementary Materials.

spectroscopy (EELS) as well as an Oxford Instruments X-Max $80 \mathrm{~mm}^{2}$ detector for energy-dispersive X-ray (EDX) data acquisition. During the scanning TEM (STEM) investigations with an approximate probe size of $1.3 \AA$ according to the instrument's specifications, the beam current was tuned to $42 \mathrm{pA}$ for imaging and 100-150 pA for EELS and EDX mapping. The acceptance semi-angle of the spectrometer was set to $39 \mathrm{mrad}$ and the inner and outer collection semi-angle of the included annular dark-field (ADF) detector to $46.8 \mathrm{mrad}$ and $200 \mathrm{mrad}$. EELS and EDX data were analyzed with HyperSpy [9] and all windows used for background subtraction and signal integration are summarized in Tab. S2.

\section{Pristine homogeneous thin film preparation}

The general framework of the presented preparation technique will be described in this section by the example of a $100 \mathrm{~nm}$ thick $\mathrm{Pr}_{1-x} \mathrm{Ca}_{x} \mathrm{MnO}_{3}$ (PCMO) epitaxial film with $x=0.34$, which has been deposited via ion beam sputtering on a $\mathrm{SrTi}_{1-y} \mathrm{Nb}_{y} \mathrm{O}_{3}$ (STNO) substrate with $y=0.002$ as it has been described in Ref. [10]. Subsequently, as sketched in Fig. 1(a), the sample was mounted upside down on a stainless steel cylinder using QuickStick 135 Mounting Wax and ground to a thickness of approximately $20 \mu \mathrm{m}$ with a PRESI Minitech 233 disc grinder and P2400 grinding paper. The wax was removed in an acetone bath and the specimen was glued upside down with silver paint on a scanning electron microscope (SEM) stub such that the region of interest sticks over the stub's edge. Please note that Fig. 1(b) sketches the sample on a slotted TEM grid instead allowing for easy flipping as it will be used in Section 3.2 for the sake of site-specific preparation.

After insertion into the FIB, four regular cross sections (following the FEI terminology) with dimensions $20 \times 14 \times 50 \mu \mathrm{m}^{3}$ and a beam current of $47 \mathrm{nA}$ were dug from the backside to create the outer trenches with fourfold symmetry in Fig. 1(c). Please note that the indicated depth of the regular cross section is much larger than the sample thickness because the value is calibrated to silicon with a significantly higher sputtering rate compared to STNO. In a second step, the inner cut-out frame in Fig. 1(c) was created employing rectangular patterns at $47 \mathrm{nA}$. In the lower right part, a small bridge was left until the central block was attached to the EasyLift ${ }^{\mathrm{TM}}$ nanomanipulator. Finally, after lifting out and attaching the block to an Omniprobe support grid with 


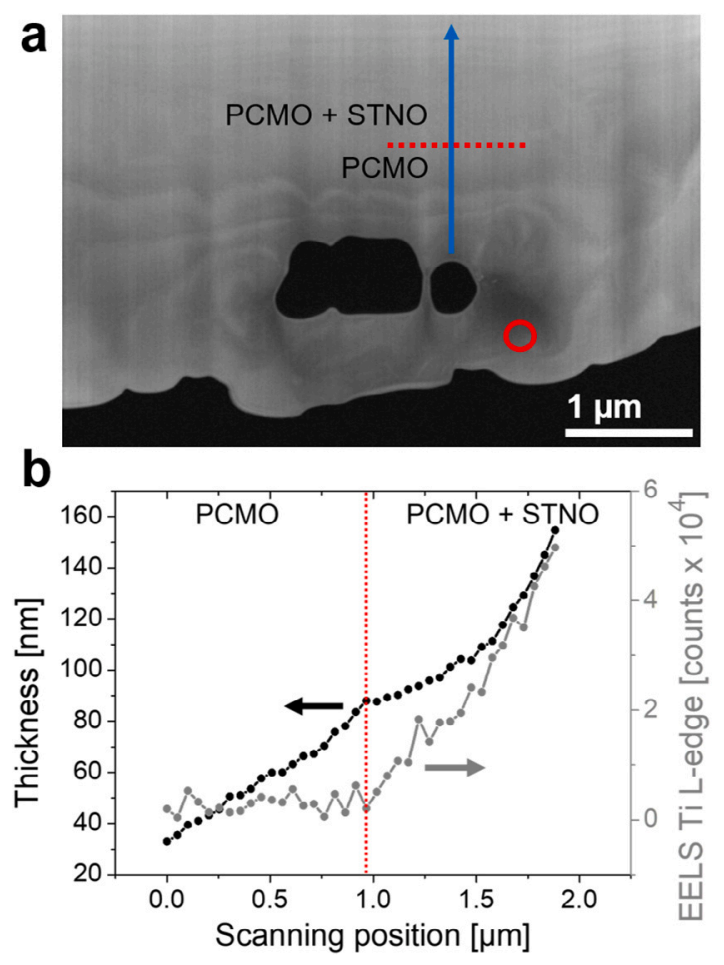

Fig. 3. (a) ADF-STEM overview of the thin region in Fig. 1(f). The blue arrow indicates the profile along which the EELS data shown in (b) have been recorded. Furthermore, the red circle marks the region investigated by SAED and HRSTEM. (b) Thickness as well as integrated Ti L-edge intensity after power-law background subtraction along the blue arrow in (a). The red dotted lines mark the position at which substrate contributions become significant. The windows used for background subtraction and signal integration are summarized in Tab. S2.

platinum as shown in Fig. 1(d), a $15 \mu \mathrm{m}$ wide and $50 \mu \mathrm{m}$ deep cleaning cross section was first used with $21 \mathrm{nA}$ and subsequently with $2.5 \mathrm{nA}$ to thin the block to electron transparency. A sketched side-view during thinning as well as the final lamella are shown in Fig. 1(e) and (f). The red arrow indicates the ion milling direction. Importantly, the sample was intentionally tilted such that the angle between the ion beam and the surface amounts to $15^{\circ}$ to maintain a rather thick part at the top guaranteeing mechanical stability during the substrate removal. The stage and nanomanipulator positions during all relevant FIB steps using a $38^{\circ}$ pretilted holder are illustrated in Fig. 2.

\subsection{Plan-view TEM investigations}

The resulting lamella was investigated in the TEM by means of ADFSTEM combined with EELS as well as selective area electron diffraction (SAED) to assess its crystalline quality. Fig. 3(a) shows an ADF-STEM overview of the thin part of the specimen including a blue arrow resp. a red circle marking the areas analyzed with EELS resp. SAED as well as high-resolution STEM (HRSTEM). The results of the former are presented in Fig. 3(b) showing both the corresponding thickness as well as the integrated counts of the Ti L-edge after power-law background subtraction. Please note that $\lambda_{\mathrm{PCMO}}=110 \mathrm{~nm}$ [11] was used to obtain absolute thicknesses following Malis' method [12] yielding incorrect values in regions with remaining STNO substrate. The point along the line profile where substrate contributions get significant is marked with a dotted red line. In fact, the kink in the thickness curve caused by the change in lambda (and possibly the thinning behavior) is both consistent with the emergence of finite Ti L-edge counts as well as the nominal film thickness. The area of free-standing PCMO amounts to approximately $20 \mu \mathrm{m}^{2}$. As a first confirmation that the sample is sufficiently clean for TEM investigation, i.e. no significant redeposited a
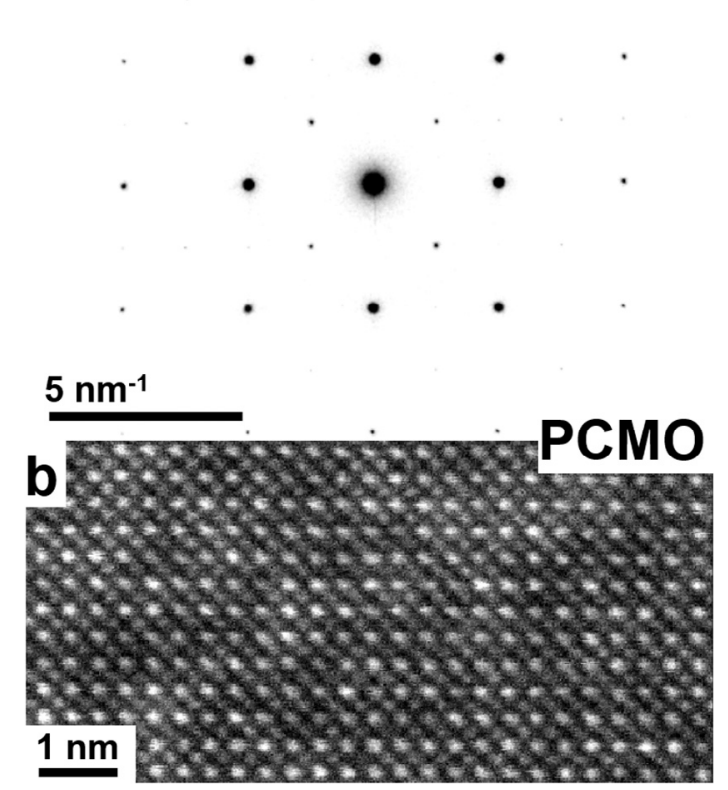

Fig. 4. SAED pattern (a) as well as ADF-STEM acquisition (b) of the region marked with the red circle in Fig. 3 showing high crystalline quality and no significant signal from contamination, e.g., amorphous rings, or disordered layers.

material or residual wax is present at the surface, an SAED pattern as well as an HRSTEM image of the thin area $(t \approx 20 \mathrm{~nm})$ marked with the red circle in Fig. 3(a) are shown in Fig. 4(a) resp. (b). Even though the contrast in (a) is digitally enhanced as can be inferred from the spurious intensity around the direct beam, no significant contribution of amorphous or poly-crystalline contaminants is observed. Consequently, the prepared free-standing film is well-suitable for HRSTEM imaging as demonstrated in Fig. 4(b). Furthermore, it is worth mentioning that in case materials exhibiting redeposition exist, the presented method could be easily combined with soft surface protection layers which can be removed by plasma-cleaning subsequently or temporary cover blocks as demonstrated on cross-sectional lamellae in Ref. [13] and [14].

\subsection{Assessment of surface quality by cross-sectional slice extraction}

In order to further assess the quality of both the pristine as well as the ion beam treated surface, HRSTEM and EELS investigations of a cross-sectional slice extracted from a plan-view lamella shall be presented. Please note that a final $5 \mathrm{kV}$ thinning step using a beam current of $15 \mathrm{pA}$ and a tilt angle of $19^{\circ}$ was performed during planview preparation in this section. An overview of the slice extraction is given in Fig. 5(a). Importantly, after the final thinning step of the plan-view preparation as well as reactive Ar-O plasma cleaning for one minute and before the slice extraction, the pristine surface was thermally evaporated with an approximately $100 \mathrm{~nm}$ thick $\mathrm{Al}$ film at room temperature to cover the state after plan-view preparation. The pressure during $\mathrm{Al}$ evaporation was $5 \times 10^{-5} \mathrm{mbar}$. The ion beam treated surface was covered with a Pt bar deposited with the electron beam using $2 \mathrm{kV}$ as shown in Fig. 5(a). The extracted cross-sectional slice was thinned to electron transparency using an acceleration voltage of $5 \mathrm{kV}$, a beam current of $15 \mathrm{pA}$, and a tilt angle of $\pm 5^{\circ}$ during the final thinning step.

Fig. 5(b) shows an ADF-STEM overview of the resulting crosssection. The blue and red rectangles mark regions of the ion beam treated and pristine surfaces which were investigated by HRSTEM and 

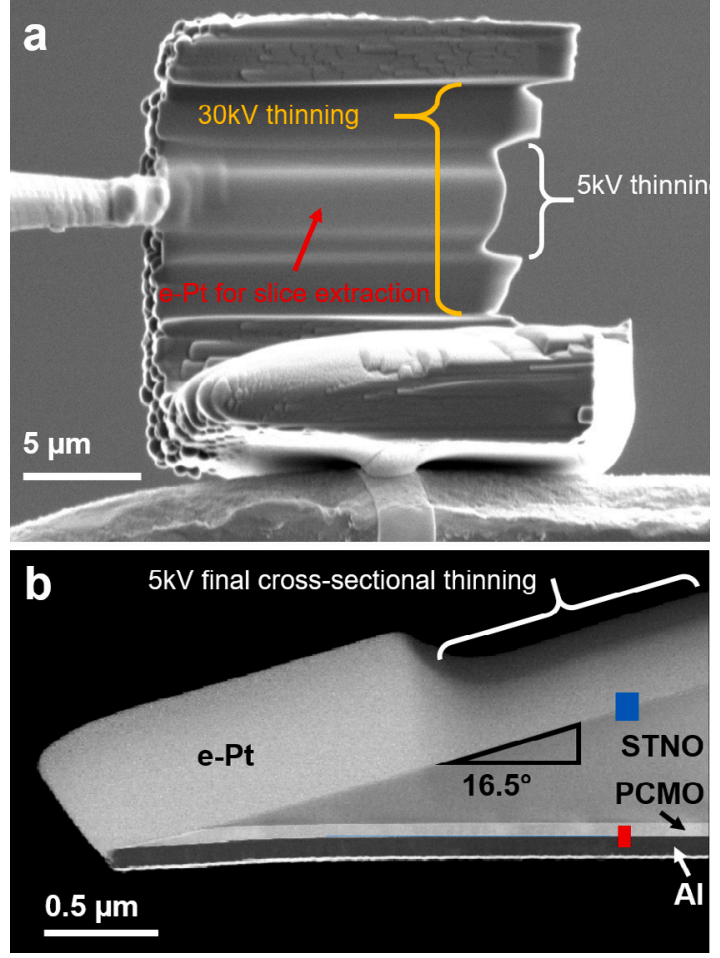

Fig. 5. (a) Slice extraction from a final plan-view lamella: The pristine surface was thermally evaporated with approximately $100 \mathrm{~nm} \mathrm{Al} \mathrm{after} \mathrm{final} \mathrm{thinning} \mathrm{of} \mathrm{the} \mathrm{plan-}$ view preparation as well as plasma cleaning and before slice extraction. The e-Pt protection bar on the front side, which is attached to the nanomanipulator on the left, was deposited with the electron beam using an acceleration voltage of $2 \mathrm{kV}$. (b) ADF-STEM overview of the extracted slice revealing a well-defined wedge angle of $16.5^{\circ}$ in regions with thick remaining substrate and bending in regions where the substrate is almost entirely removed. The blue and red rectangles correspond to the surface regions presented in Figs. 6 and 7, respectively. (For interpretation of the references to color in this figure legend, the reader is referred to the web version of this article.)

EELS. The final thinning window was intentionally set with a certain distance to the tip to avoid potential bending during cross-sectional milling when approaching low cross-sectional thickness allowing for investigation of the plan-view preparation induced bending in the region where the substrate is almost or even fully removed. Nevertheless, high magnification images of the relevant surfaces in these regions exhibit no significant differences (apart from the reduced image quality due to increased cross-sectional sample thickness). In fact, both surfaces show very homogeneous behavior hence the regions marked with the blue and red rectangle are good representatives to assess their quality. Noteworthy, in regions with thick remaining substrate a well-defined wedge-angle of $16.5^{\circ}$ is observed, which is between the tilt angles of $15^{\circ}$ and $19^{\circ}$ of the two final thinning steps of the plan-view preparation. Approaching the tip to the left, i.e., the part which is of major interest for plan-view investigations, a total bend of approximately $4^{\circ}$ occurs along a distance of approximately $1 \mu \mathrm{m}$ corresponding to a mean radius of curvature of $14 \mu \mathrm{m}$. Importantly, similarly grown epitaxial thin films have been investigated in Ref. [15] showing approximately $-0.5 \%$ out-of-plane compressive strain suggesting that the bending is intrinsically caused. In fact, conventionally prepared samples of the used material system are prone to crack formation in the electron transparent areas which could be circumvented here, possibly due to the large wedge angle.

Fig. 6(a) shows an ADF-STEM image of the ion beam treated STNO surface covered with electron-deposited Pt and marked with the blue rectangle in Fig. 5(b). The yellow arrow indicates the position of the EELS line profile presented in Fig. 6(b) including the Sr L- (black), C
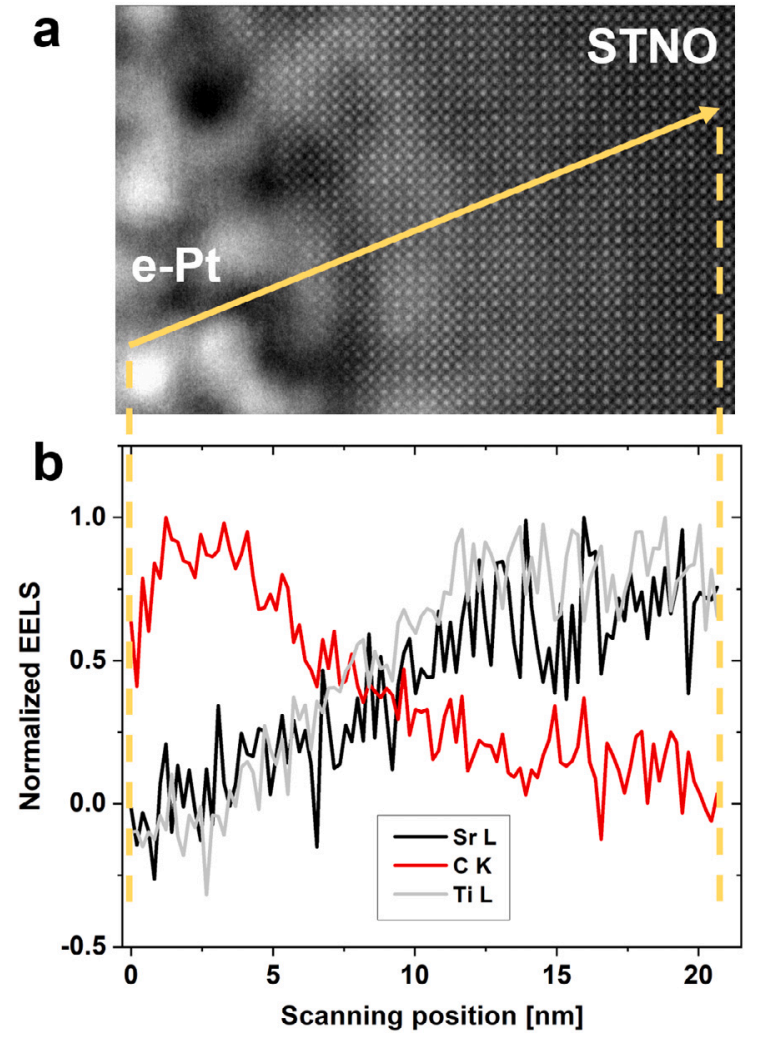

Fig. 6. (a) ADF-STEM image of the ion beam treated STNO surface covered with electron beam deposited Pt and marked with the blue rectangle in Fig. 5(b). The yellow arrow indicates the position of the EELS line profile presented in (b) including the Sr L(black), C K- (red), and Ti L-edge signal (gray) after power-law background subtraction each normalized by their maximal value. The windows used for background subtraction and signal integration are summarized in Tab. S2. (For interpretation of the references to color in this figure legend, the reader is referred to the web version of this article.)

K- (red), and Ti L-edge signal (gray) after power-law background subtraction each normalized by their maximal value. Crystalline contrast is right visible at the Sr L- and Ti L-edge onset, however, both the spotty bright contrasts in the STNO surface region as well as the transient C K-edge signal of the Pt-C alloy up to scanning positions between 10$15 \mathrm{~nm}$ indicate that the ion beam treated surface is rather textured with peak to valley distances above $5 \mathrm{~nm}$. Furthermore, it is likely that many point defects are created by the ion beam in this region. Thus, after $5 \mathrm{kV}$ thinning, amorphization of the surface region seems to be a minor effect compared with the resulting roughness and potential point defect creation.

The pristine PCMO surface covered with thermally evaporated $\mathrm{Al}$ and marked with the red rectangle in Fig. 5(b) is shown in Fig. 7(a) revealing a sharp termination of crystalline contrast. The EELS data presented in Fig. 7(b) and (c) results from a rectangular scanning region including five horizontal lines in the region marked with dashed gray lines, which were vertically averaged. While the summed spectra for energy losses above the Pr M-edge (after power-law background subtraction between $870-920 \mathrm{eV}$ ) for the regions (i) to (iv) are given in (b), the normalized EELS profiles of the Pr M- (black), C K- (red), $\mathrm{Al} \mathrm{K-} \mathrm{(green),} \mathrm{Mn} \mathrm{L-} \mathrm{(blue),} \mathrm{and} \mathrm{O} \mathrm{K-edge} \mathrm{(yellow)} \mathrm{are} \mathrm{shown} \mathrm{in} \mathrm{(c).}$ It is important to mention that the Pr M-edge strongly overlaps with the $\mathrm{Cu}$ L-edge, which is expected to contribute to the signal in case of redeposition of sputtered material of the TEM grid. In fact, as can be inferred from the characteristic white lines of the Pr M-edge, the finite signal below the $\mathrm{Al} \mathrm{K}$-edge in (iii) is caused by $\mathrm{Cu}$ instead of $\mathrm{Pr}$ proving that detectable redeposition has taken place. Furthermore, a small shoulder caused by $\mathrm{Cu}$ is visible in (ii) and spurious $\mathrm{Ga}$ L-edge 
a

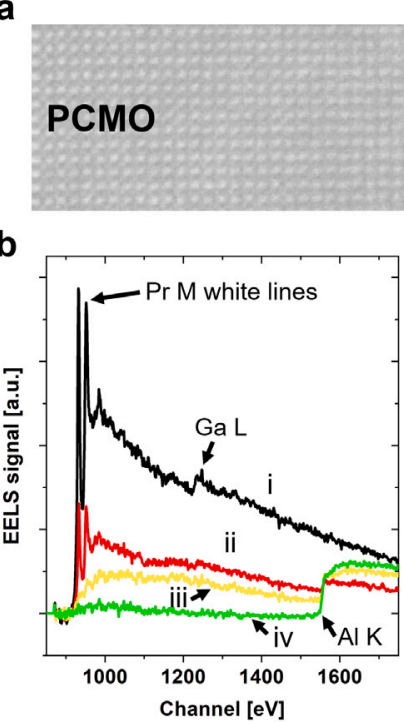

i ii iii iv

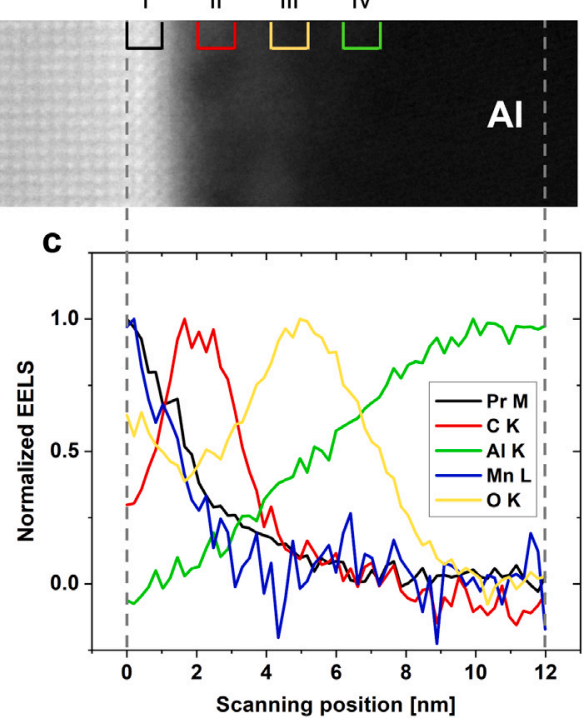

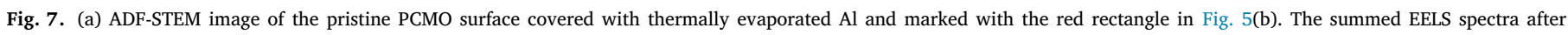
power-law background subtraction between $870-920 \mathrm{eV}$ for the ranges (i) to (iv) marked in (a) are given in (b). (c) EELS signal of the Pr M- (black), C K- (red), Al K- (green),

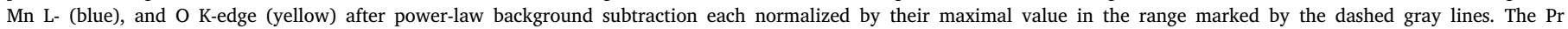

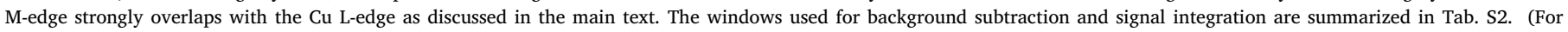
interpretation of the references to color in this figure legend, the reader is referred to the web version of this article.)
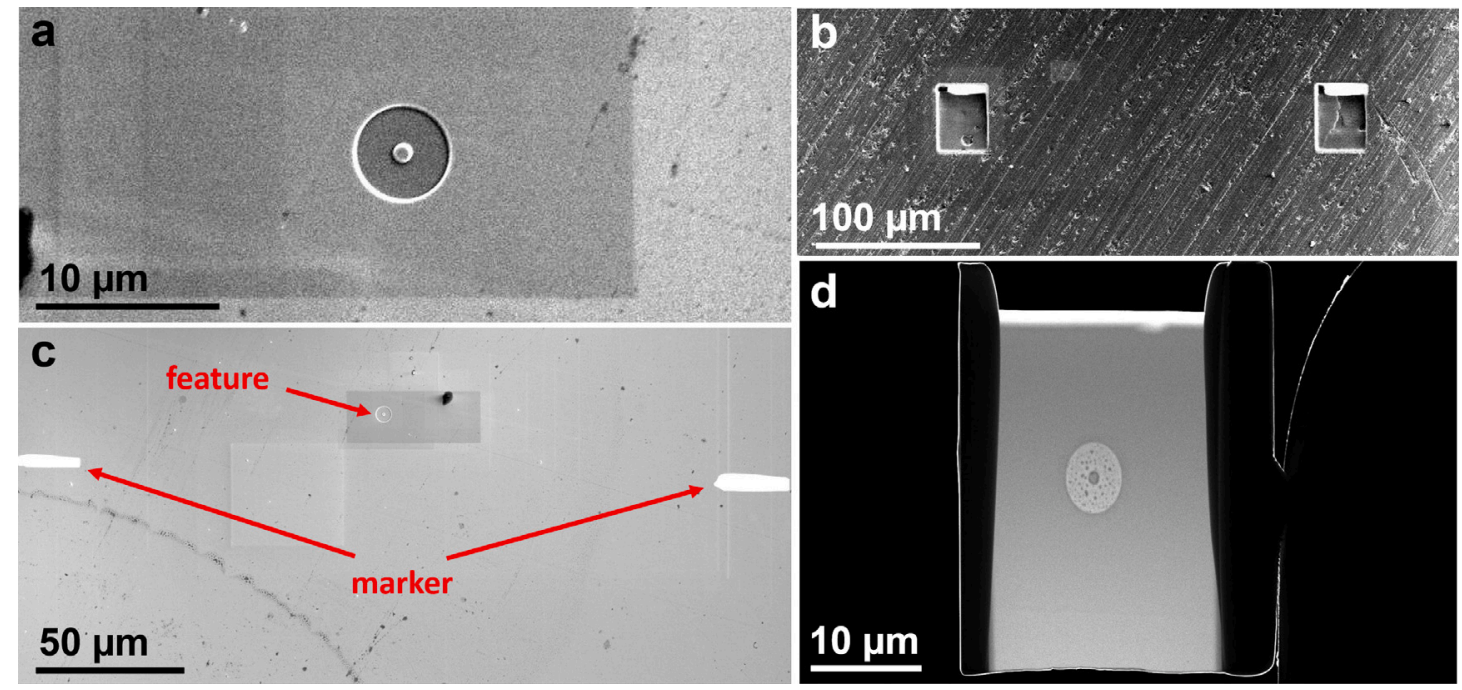

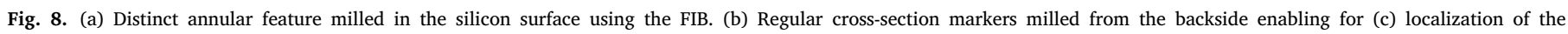

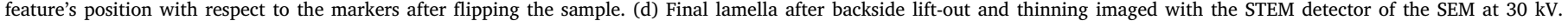

intensity caused by the Ga ion beam is found right at the surface in (i). These findings are fully consistent with the results obtained by EDX and included in Fig. S2 in the Supplementary Materials. Considering the light elements included in Fig. 7(c), a clear C and O peak is found. Since $\mathrm{Al}$ is known to form a thin native oxide layer under the conditions used during evaporation [16], the latter is expected and most likely not caused by the plan-view preparation process. The origin of the former, however, is less tangible since it could be caused by residual wax, contamination in the FIB, or simply the exposure of the surface to air after plasma cleaning and prior to $\mathrm{Al}$ evaporation. Lacking a definite proof, we refrain from claiming the last option to be the ultimate cause of the observed C peak. Still, since plasma cleaning is known to be an effective measure to remove carbon contaminations caused by the former two options, it serves as a possible explanation.

\section{Site-specific preparation with large field of view}

In order to prove that the suggested method is not only suitable for extraction of homogeneous materials but can be conducted in a sitespecific manner as well, a second example involving a distinct feature at the surface shall be presented. Furthermore, its applicability to another material, i.e. silicon, as well as the scalability to large fields of view will be demonstrated.

Analogously to the mechanical preparation above, an initially $1 \mathrm{~mm}$ thick silicon waver has been ground to a thickness of approximately $20 \mu \mathrm{m}$. Subsequently, the specimen was glued across a $2 \times 1 \mathrm{~mm}^{2}$ copper slot enabling the observations in the FIB from the front- and backside. To create a distinct feature at the surface, a $150 \mathrm{~nm}$ deep annular pattern with an inner resp. outer diameter of 1 resp. $5 \mu \mathrm{m}$ was dug with the ion beam as shown in Fig. 8(a). Subsequently, the following three-step procedure was conducted to extract the unique feature: Firstly, two coarsely-positioned markers (regular cross-sections) 
were milled with the ion beam from the backside (Fig. 8(b)). Secondly, the sample was flipped and solely observed with the electron beam to locate the position of the surface feature with respect to the markers (Fig. 8(c)). Lastly, after flipping the specimen again, the site of interest was extracted by exploiting the information obtained in the previous step. Here, in order to prove the comparatively easy preparation of large lamellae, a block with a $30 \times 30 \mu \mathrm{m}^{2}$ surface area was transferred and the angle between ion beam and surface during thinning was decreased to $3^{\circ}$. Fig. 8(d) shows a high-angle annular dark-field (HAADF) image of the final lamella recorded with the STEM detector included in the SEM at an acceleration voltage of $30 \mathrm{kV}$. Obviously, the donut-shaped site of interest is well-located in the extracted block. In fact, comparing its central position to the geometrical mean of the four corners of the approximately $600 \mu \mathrm{m}^{2}$ large electron transparent area results in a distance of only $1.3 \mu \mathrm{m}$.

\section{Summary and conclusion}

In this paper, we have demonstrated that the combination of mechanical grinding and backside lift-out in the FIB is well-suitable for plan-view lamellae extraction of pristine surfaces and meets many requirements of TEM sample preparation simultaneously: Sticking to the categorization mentioned above, the suggested procedure clearly circumvents the site-specifity restrictions of group (i) methods as has been demonstrated by the extraction of a distinct surface feature with micrometer precision. In addition, it enables straight-forward lamellae fabrication with a large field of view since the sputtering volume scales simply with the circumference of the extracted block, i.e. with the square of the edge for quadratic fields of view. In contrast, using category (ii) methods and thus a frontside lift-out, the milling depth in the FIB scales with the edge length resulting in a sputtering volume proportional to the cube of the edge. Consequently, the presented approach combines the benefits of both groups adding value to existing strategies of plan-view TEM lamellae preparation.

The resulting quality of both the pristine front surface as well as the ion beam treated back surface was carefully assessed by TEM investigations of an extracted cross-sectional slice showing that the latter exhibits a certain roughness, which is typical for ion beam treated surfaces. In contrast, the former does not suffer any ion beam damages and the surface contamination caused by the preparation (or intermediate exposure to air) was found to be a 3-4 nm thick layer consisting mainly of carbon with small resp. spurious contributions of copper and gallium.

\section{CRediT authorship contribution statement}

Tobias Meyer: Original idea was conceived, Polishing, FIB preparation, TEM investigation of the samples, Writing - original draft. Tobias Westphal: Polishing, FIB preparation, TEM investigation of the samples, Writing - original draft. Birte Kressdorf: Thin film perovskite sample preparation. Ulrich Ross: Polishing, FIB preparation, TEM investigation of the samples. Christian Jooss: Revision. Michael Seibt: Revision.

\section{Declaration of competing interest}

The authors declare that they have no known competing financial interests or personal relationships that could have appeared to influence the work reported in this paper.

\section{Acknowledgments}

The project was funded by the Deutsche Forschungsgemeinschaft (DFG, German Research Foundation) project no. SE 560-6/1 as well as $217133147 /$ SFB 1073, projects B02, Z02. The use of equipment in the "Collaborative Laboratory and User Facility for Electron Microscopy" (CLUE, Göttingen) is gratefully acknowledged.

\section{Appendix A. Supplementary data}

Supplementary material related to this article can be found online at https://doi.org/10.1016/j.ultramic.2021.113320. An illustration of suitable stage positions for untilted holders, a detailed list of employed FIB patterns, background and signal windows used in the EELS and EDX analysis, as well as an EDX line profile of the pristine surface is provided.

\section{References}

[1] L.A. Giannuzzi, F.A. Stevie, A review of focused ion beam milling techniques for tem specimen preparation, Micron 30 (3) (1999) 197-204.

[2] F. Lenrick, M. Ek, D. Jacobsson, M.T. Borgström, L.R. Wallenberg, Fib plan and side view cross-sectional tem sample preparation of nanostructures, Microsc. Microanal. 20 (1) (2014) 133-140.

[3] F. Stevie, R. Irwin, T. Shofner, S. Brown, J. Drown, L. Giannuzzi, Plan view tem sample preparation using the focused ion beam lift-out technique, in: AIP Conference Proceedings, Vol. 449, American Institute of Physics, 1998, pp. 868-872.

[4] R. Anderson, S.J. Klepeis, Combined tripod polishing and fib method for preparing semiconductor plan view specimens, MRS Online Proc. Libr. Arch. 480 (1997).

[5] K. O'Shea, D. McGrouther, C. Ferguson, M. Jungbauer, S. Hühn, V. Moshnyaga, D. MacLaren, Fabrication of high quality plan-view tem specimens using the focused ion beam, Micron 66 (2014) 9-15.

[6] R. Langford, Y. Huang, S. Lozano-Perez, J. Titchmarsh, A. Petford-Long, Preparation of site specific transmission electron microscopy plan-view specimens using a focused ion beam system, J. Vacuum Sci. Technol. B: Microelectron. Nanometer Struct. Process. Meas. Phenomena 19 (3) (2001) 755-758.

[7] M. Jublot, M. Texier, Sample preparation by focused ion beam micromachining for transmission electron microscopy imaging in front-view, Micron 56 (2014) 63-67.

[8] C. Li, G. Habler, L.C. Baldwin, R. Abart, An improved fib sample preparation technique for site-specific plan-view specimens: A new cutting geometry, Ultramicroscopy 184 (2018) 310-317.

[9] F. de la Peña, E. Prestat, V.T. Fauske, P. Burdet, P. Jokubauskas, M. Nord, T. Ostasevicius, K.E. MacArthur, M. Sarahan, D.N. Johnstone, J. Taillon, J. Lähnemann, V. Migunov, A. Eljarrat, J. Caron, T. Aarholt, S. Mazzucco, M. Walls, T. Slater, F. Winkler, pquinn dls, B. Martineau, G. Donval, R. McLeod, E.R. Hoglund, I. Alxneit, D. Lundeby, T. Henninen, L.F. Zagonel, A. Garmannslund, Hyperspy/hyperspy: Hyperspy v1.5.2, 2019, http://dx.doi.org/10.5281/zenodo. 3396791.

[10] B. Ifland, J. Hoffmann, B. Kressdorf, V. Roddatis, M. Seibt, C. Jooss, Contribution of jahn-teller and charge transfer excitations to the photovoltaic effect of manganite/titanite heterojunctions, New J. Phys. 19 (6) (2017) 063046.

[11] J. Norpoth, S. Mildner, M. Scherff, J. Hoffmann, C. Jooss, In situ tem analysis of resistive switching in manganite based thin-film heterostructures, Nanoscale 6 (16) (2014) 9852-9862.

[12] T. Malis, S. Cheng, R. Egerton, Eels log-ratio technique for specimen-thickness measurement in the tem, J. Electron Microsc. Tech. 8 (2) (1988) 193-200.

[13] V. Roddatis, G. Lole, C. Jooss, In situ preparation of $\mathrm{pr}_{1-x} \mathrm{ca}_{x} \mathrm{mno}_{3}$ and $\mathrm{la}_{1-x} \mathrm{Sr}_{x} \mathrm{mno}_{3}$ catalysts surface for high-resolution environmental transmission electron microscopy, Catalysts 9 (9) (2019) 751.

[14] C. Flathmann, H. Spende, T. Meyer, P. Peretzki, M. Seibt, Preparation techniques for cross-section transmission electron microscopy lamellas suitable for investigating in situ silicon-aluminum alloying at grain boundaries in multicrystalline silicon, Phys. Status Solidi (a) 216 (17) (2019) 1900308.

[15] B. Ifland, J. Hoffmann, T. Kramer, M. Scherff, S. Mildner, C. Jooss, Strain driven phase decomposition in ion-beam sputtered pr1- xcaxMnO3 films, J. Nanomater. 2015 (2015).

[16] N. Cai, G. Zhou, K. Müller, D.E. Starr, Effect of oxygen gas pressure on the kinetics of alumina film growth during the oxidation of al (111) at room temperature, Phys. Rev. B 84 (12) (2011) 125445. 


\title{
Site-specific plan-view TEM lamella preparation of pristine surfaces with a large field of view - Supplementary Materials
}

\author{
Tobias Meyer ${ }^{\mathrm{a}}$, Tobias Westphal ${ }^{\mathrm{a}}$, Birte Kressdorf ${ }^{\mathrm{b}}$, Ulrich Ross ${ }^{\mathrm{b}}$, Christian Jooss $^{\mathrm{b}}$, Michael Seibt ${ }^{\mathrm{a}}$ \\ ${ }^{a}$ 4th Institute of Physics - Solids and Nanostructures, University of Goettingen, Friedrich-Hund-Platz 1, 37077 Göttingen, \\ Germany \\ ${ }^{b}$ Institute of Materials Physics, University of Goettingen, Friedrich-Hund-Platz 1, 37077 Göttingen, Germany
}

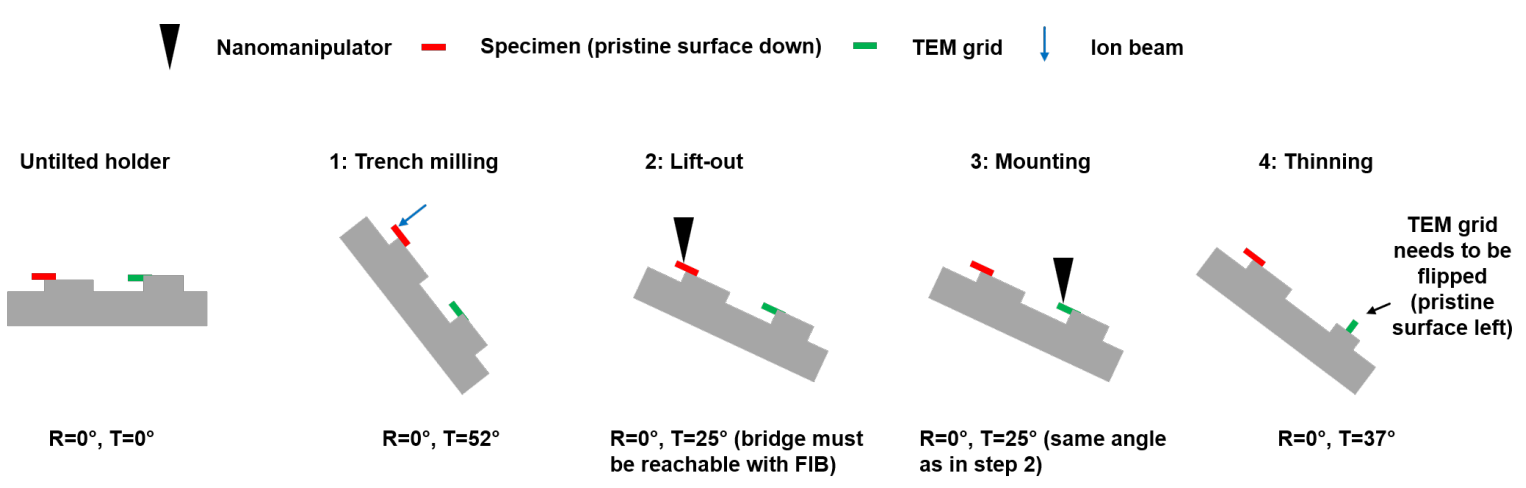

Figure S1: Illustration of the stage rotation and tilt as well as nanomanipulator position for all relevant steps in the FIB using an untilted holder. The TEM grid needs to be flipped between step 3 and 4, i.e. the holder needs to be removed and reinserted.

\begin{tabular}{ccccc} 
Step & $\begin{array}{c}\text { Stage tilt } \\
\text { pre- } / \text { untilted }\end{array}$ & Pattern type & $\begin{array}{c}\text { Pattern size }[\mathrm{\mu m}] \\
(x \times y \times z)\end{array}$ & $\begin{array}{c}\text { Ion beam } \\
\text { current [nA] }\end{array}$ \\
\hline trench milling part 1 & $52^{\circ}$ & regular cross-section & $20 / 30 \times 14 \times 50$ & 47 \\
trench milling part 2 & $52^{\circ}$ & rectangle & $18 / 4 \times 2 \times 10$ & 47 \\
lift-out, Pt attachment & $38^{\circ} / 25^{\circ}$ & Pt-dep & $\sim 1 \times 1 \times z$ & 0.08 \\
lift-out, bridge cutting & $38^{\circ} / 25^{\circ}$ & rectangle & $\sim 1 \times 5 \times z$ & 0.23 \\
mounting, Pt attachment & $0^{\circ} / 25^{\circ}$ & Pt-dep & $\sim 1 \times 1 \times z$ & 0.08 \\
mounting, cut-off & $0^{\circ} / 25^{\circ}$ & rectangle & $\sim 0.5 \times 2 \times z$ & 0.23 \\
$30 \mathrm{kV}$ thinning & $15^{\circ} / 37^{\circ}$ & cleaning cross-section & $15 \times y \times 50-100$ & $47 \rightarrow 21 \rightarrow 2.5$ \\
$5 \mathrm{kV}$ thinning (Sec. 4) & $19^{\circ} / 33^{\circ}$ & dynamic all directions & $5 \times y \times 0.1$ & 0.015 \\
\hline
\end{tabular}

Table S1: Summary of all FIB patterns employed during plan-view preparation including the stage tilt, pattern type and size, as well as used beam current. Except for the last $5 \mathrm{kV}$ thinning step, which was only performed for the lamella presented in Sec. 4, all other patterns were used at an ion beam acceleration voltage of $30 \mathrm{kV}$. The larger $x$ size of $30 \mu \mathrm{m}$ for the trench milling part 1 refers to the Si example presented in Sec. 5 and the two $x$ sizes for the trench milling part 2 correspond to the edge and corner parts visible in Fig. 1 (c). The $y$ size of the two last steps needs to be adjusted to the thickness of the block extracted from the backside. The $z$ size of the lift-out and mounting steps is not fixed as the patterns were stopped manually when the Pt weld appeared sufficiently stable resp. remaining bridges were fully cut. 


\begin{tabular}{ccc} 
EELS & Background window $[\mathrm{eV}]$ & Signal window $[\mathrm{eV}]$ \\
\hline Ti L-edge & $400-430$ & $440-490$ \\
Sr L-edge & $1800-1900$ & $1900-2100$ \\
C K-edge & $220-260$ & $270-320$ \\
Pr M-edge & $870-920$ & $920-1020$ \\
Al K-edge & $1430-1530$ & $1550-1650$ \\
Mn L-edge & $600-630$ & $640-740$ \\
O K-edge & $460-510$ & $520-570$ \\
\hline EDX & Background window (pre and post line) $[\mathrm{keV}]$ & Signal window $[\mathrm{keV}]$ \\
\hline $\mathrm{Al} \mathrm{K} \alpha$ & $1.25-1.35$ and $1.61-1.71$ & $1.35-1.61$ \\
$\mathrm{Ca} \mathrm{K} \alpha$ & $3.45-3.55$ and $3.85-3.95$ & $3.55-3.85$ \\
$\mathrm{Cu} \mathrm{K} \alpha$ & $7.8-7.85$ and $8.3-8.35$ & $7.85-8.3$ \\
$\mathrm{Ga} \mathrm{K} \alpha$ & $9.1-9.15$ and $9.3-9.35$ & $9.15-9.3$ \\
$\mathrm{Mn} \mathrm{K} \alpha$ & $5.69-5.73$ and $6.1-6.2$ & $5.73-6.1$ \\
$\mathrm{Pr} \mathrm{K} \alpha$ & $4.75-4.8$ and $5.25-5.3$ & $4.8-5.25$ \\
$\mathrm{Pt} \mathrm{K} \alpha$ & $9.3-9.35$ and $9.55-9.6$ & $9.35-9.55$ \\
\hline
\end{tabular}

Table S2: Windows used for power-law (EELS) resp. Bremsstrahlung (EDX) background subtraction and signal integration.

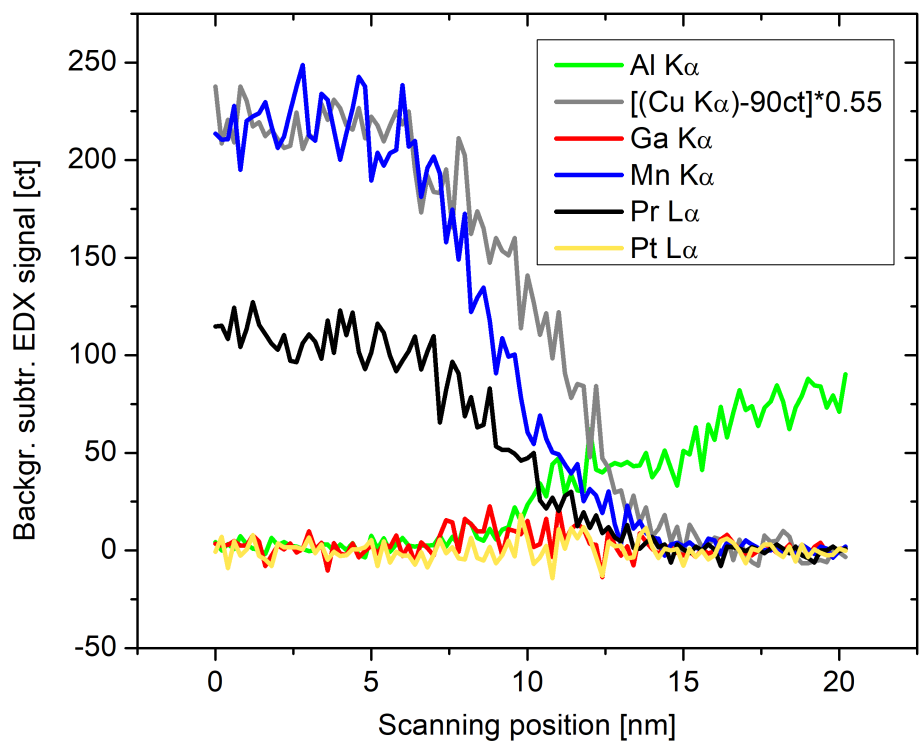

Figure S2: EDX signal of the $\mathrm{Al} \mathrm{K} \alpha$ (green), $\mathrm{Cu} \mathrm{K} \alpha$ (gray), Ga K $\alpha$ (red), Mn K $\alpha$ (blue), Pr L $\alpha$ (black), and Pt L $\alpha$ (yellow) line across the pristine PCMO surface thermally evaporated with $\mathrm{Al}$ and marked with the red rectangle in Fig. 5 (b) after background subtraction. The windows used for background subtraction and signal integration are summarized in Tab. S2. The $\mathrm{Cu} \mathrm{K} \alpha$ counts were scaled as indicated in the legend to match the levels of the Mn K $\alpha$ counts in PCMO and Al. Due to the background signal of the $\mathrm{Cu}$ grid, no quantification was performed. 


\subsubsection{Cross-sectional Preparation for In-situ Heating and Bi- asing}

The great potential of MEMS chips as sample carriers enabling in-situ heating and biasing experiments in the TEM has already been described in the previous chapter and will be used extensively in the following. Due to the chips' geometry, existing FIB preparation strategies have to be modified. Fig. 4.1 shows the SEM holder designs as well as stage positions used in this thesis for cross-sectional lamellas mounting in the Nova (left) resp. Helios (right) FIB. In the former instrument, a $90^{\circ}$-gableshaped holder is used with the sample (red) and chip (green) mounted on the opposite faces. This design was already presented in [126, 127] and enables cross-sectional and planview FIB treatments of both the sample as well as the chip. Furthermore, the different stage tilts result in a $15^{\circ}$ offset of the
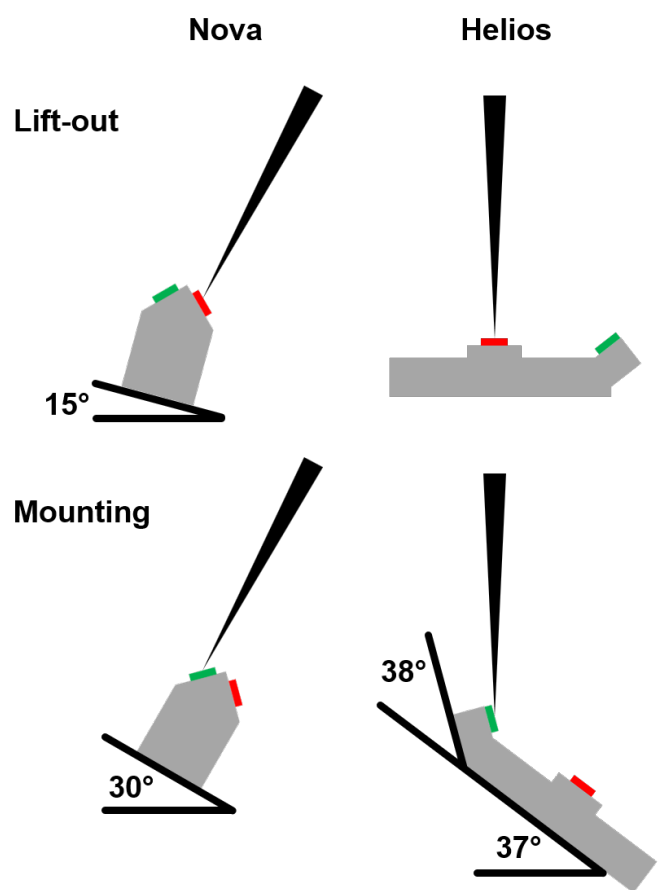

Figure 4.1: Used sample holder geometries (grey) and stage tilts for lamellas liftout (top) and mounting (bottom) in the Nova (left) resp. Helios (right) FIB. Sample, chip, and nanomanipulator are drawn in red, green, and black.

cross-sectional lamella on the

chip making both sides accessible for subsequent ion beam thinning. The complementary approach used in the Helios can be considered as an easy solution to exploit the attached load-lock for quick insertion resp. removal into resp. from the FIB chamber. As the related height restrictions prohibit a transfer of the formerly constructed gable-shaped holder through the lock, a more compact design is needed. This is realised by loading the chip (clamped on an $\mathrm{Al}$ cuboid) into the $38^{\circ}$ tilted tray of the commercial FEI holder and using the shown stage tilts of $0^{\circ}$ resp. $37^{\circ}$ yielding the same final position of the lamella on the chip. 
In addition to a successful transfer of lamellas from bulk material to MEMS chips, the preparation for biasing purposes demands both for (typically low ohmic) electric contacts at specific positions as well as prevention of short circuits. To meet the former requirement, it is thus desirable to extract lamellas including previously processed metal contact and connect these to the MEMS chip via conductive FIB$\mathrm{Pt} / \mathrm{C}$ deposition. Fig. 4.2 shows two PCMO-STNO lamellas being both contacted via an ion beam sputtered Pt (yellow) contact on top of the PCMO (red) layer but without any additional metal layer between STNO (blue) and FIB-Pt/C (green). The geometrical differences represent two different approaches to meet the second requirement, i.e. to avoid short circuits: In the top image, in accordance with [29], vertical cuts have been milled with the FIB leaving solely the electron transparent central part as electrical bridge between the contacts left and right of the lamella. Alternatively, as shown in the bottom image, an L-shaped cut can be created enabling the location of both contacts on the left side and thus a higher flexibility during thinning at the right. Resulting serial and par-
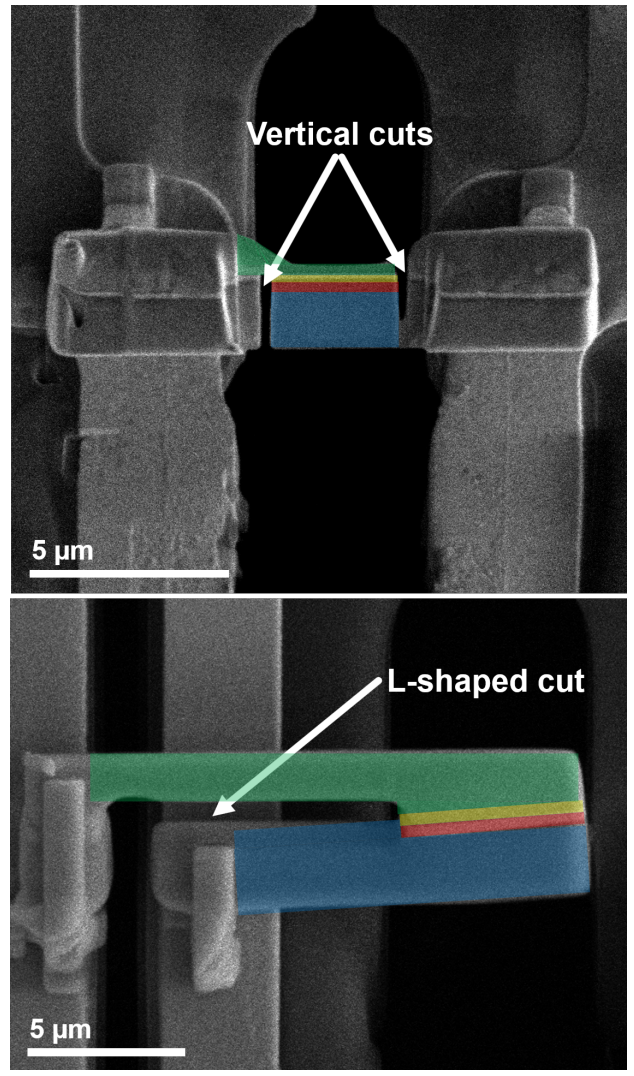

Figure 4.2: Electric contacts for biasing of PCMO-STNO lamellas are realised by contacting a previously deposited Pt metal contact (yellow) on top of the PCMO (red) resp. directly the STNO substrate (blue) with FIB$\mathrm{Pt} / \mathrm{C}$ (green). In addition, vertical (top) or L-shaped (bottom) cuts can be used to prevent short circuits leading to contacts on opposed resp. the same side of the lamella.

allel resistances of both geometries will be quantified and discussed in the context of STEM-EBIC in section 7.1 . 

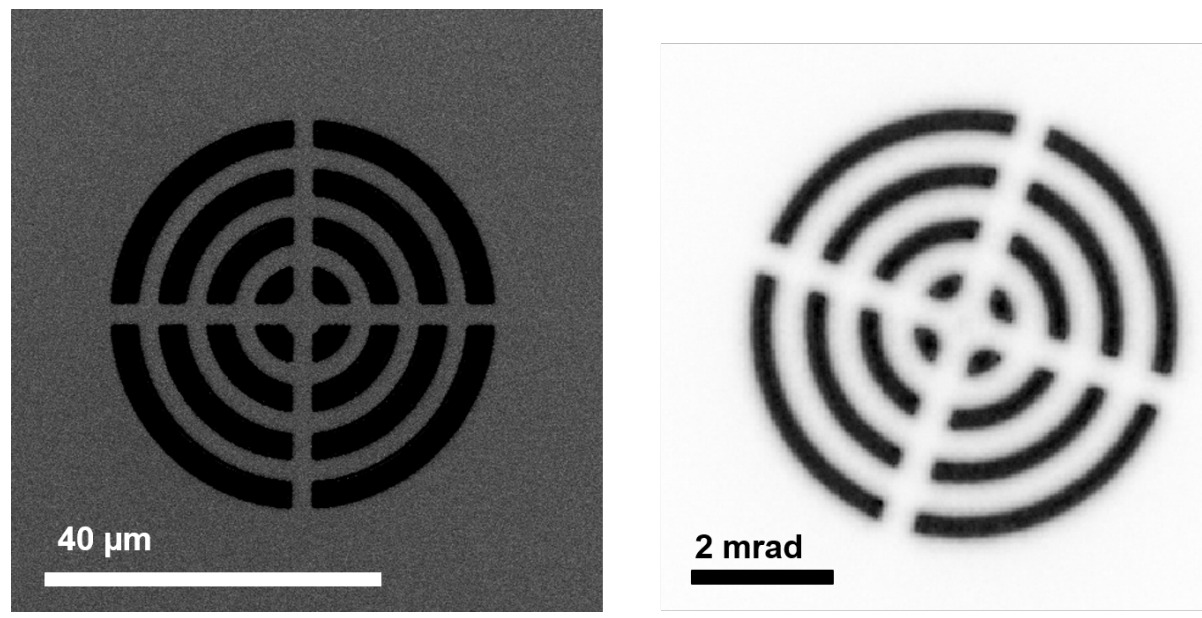

Figure 4.3: SEM overview of a bullseye aperture (left) corresponding to a 3 mrad semi-convergence angle when used as limiting third condensor aperture as well as the resulting direct beam in nano diffraction mode (right).

\subsection{Probe Shaping via a Custom Condensor Aper- ture}

Inspired by [128], a custom condensor aperture was fabricated in this work which is well-suitable for 4D-STEM strain mapping of crystalline materials. Generally, both the amplitude as well as the phase of the electron wave propagating through the condensor aperture plane can be modified by inserting strongly scattering resp. weakly interacting materials. A large variety of possible resulting probe shapes in the sample plane has been reviewed in [129]. Here, solely amplitude modifications for the sake of a precise numerical localisation of diffraction spots will be considered as it has been originally suggested in [130] and subsequently realised experimentally and improved in $[128,131,132]$. In order to reproduce the results obtained in the latter reference, an approximately $400 \mathrm{~nm}$ thick gold layer was thermally evaporated on a $200 \mathrm{~nm}$ thick PELCO ${ }^{\circledR}$ silicon nitride support film with nine $0.1 \times 0.1 \mathrm{~mm}^{2}$ windows. Subsequently, bullseye structures with radii $7.6 \mu \mathrm{m}, 15.2 \mu \mathrm{m}, 22.8 \mu \mathrm{m}, 30.4 \mu \mathrm{m}$, and $38.0 \mu \mathrm{m}$ were milled in different windows using the FIB ( $30 \mathrm{kV}$ acceleration voltage, $3 \mathrm{nA}$ beam current) resulting in semi-convergence angles of $1-5 \mathrm{mrad}$ after inserting the aperture in the third condensor plane of the Titan microscope. In fact, using the third plane due to limited space in the second is the only conceptual difference 


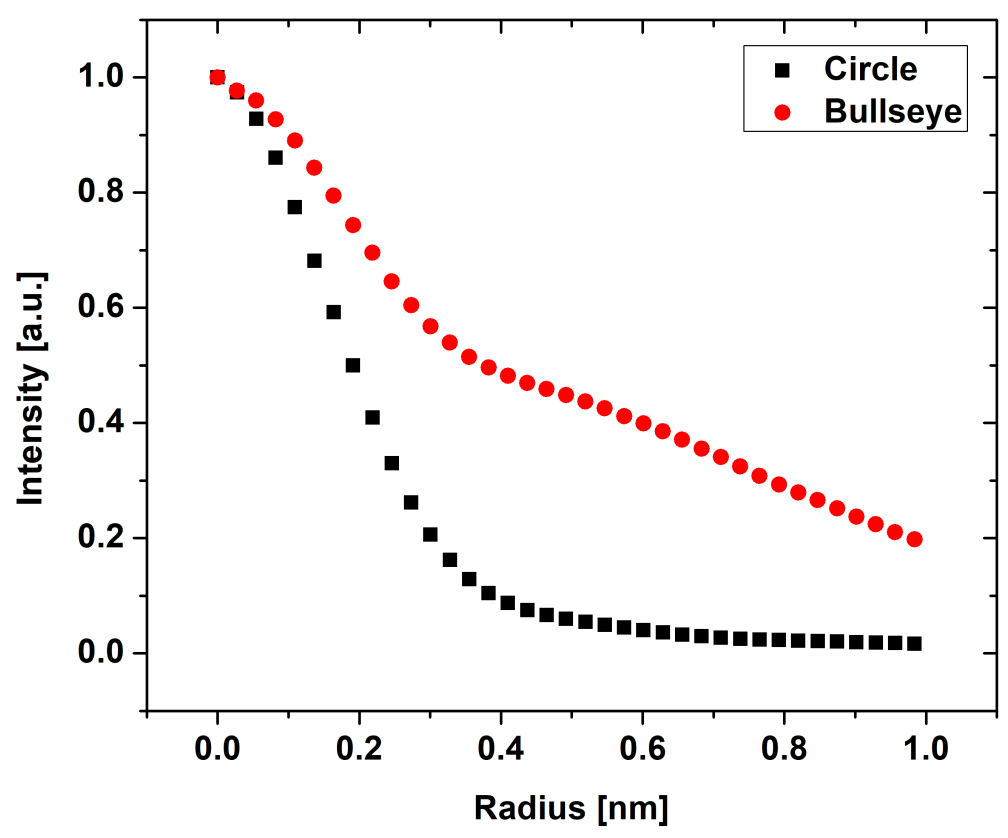

Figure 4.4: Azimuthally averaged intensity profile of the probe formed by a circular (black) resp. bullseye (red) aperture with a semi-convergence angle of $3 \mathrm{mrad}$.

compared with [128] leading to the necessity of different physical structured aperture sizes to achieve several semi-convergence angles as the latter is normally adjusted with a limiting second condensor aperture. Fig. 4.3 shows an SEM overview (left) as well as the resulting direct beam after insertion in the TEM (right) of the bullseye with a semi-convergence angle of $3 \mathrm{mrad}$.

Obviously, as the aperture blocks a significant domain of spatial frequencies, a deteriorated spatial resolution is expected. In order to compare the $3 \mathrm{mrad}$ bullseye aperture to its circular counterpart, azimuthally averaged intensity profiles normalised to their maxima are shown for both cases in Fig. 4.4 confirming the increased delocalisation of the patterned probe. The full width half maximum (FWHM) using the bullseye aperture amounts to $0.8 \mathrm{~nm}$, which is suprisingly much smaller than the value of $2.7 \mathrm{~nm}$ obtained in [128]. Still, this large difference should not be overestimated in terms of spatial resolution as the maximal intensity and thus the FWHM depends 
sensitively on defocus. The experimental prove that nanometer structures can be resolved using the structured aperture will be given in the following section in the context of 4D-STEM strain mapping.

\subsection{Implementation of 4D-STEM}

In the context of this dissertation, a software solution for both data acquisition as well as analysis of 4D-STEM data has been developed and will be described in detail in this section. The package consists of two Gatan DigitalMicrograph (DM) plugins, which enables the user to combine it with many of the latter's powerful image operation and visualisation tools.

As mentioned in subsection 3.2.5, several projects making 4D-STEM data analysis tools publicly available have been launched and published recently [107-109] and will be compared to the herein presented solution. In contrast, likely due to the complexity of different hardware combinations, general data acquisition solutions have not been presented yet and are typically part of expensive commercial packages. The acquisition plugin developed here can be run at any microscope including a Gatan DigiScan II unit as well as a camera live view accessible in DM. In addition, as will be shown through the example of live-processing, a shared architecture of acquisition and analysis routines can be very symbiotic.

Obviously, the development of such a large-scale project as well as its application to various specific problems for demonstration purposes benefits tremendously from fruitful discussion with scientific colleagues as well as demands for a lot of sample preparation and TEM experiments. Therefore, several teams have been formed to obtain the herein presented results and the reader is strongly encouraged to examine the acknowledgements, in which a detailed listing of contributors is given.

\subsubsection{D-STEM Acquisition}

In order to record 4D-STEM data stacks, the collection of digital images has to be synchronised with the scanning process. As DM offers scripting commands for both image acquisition as well as beam positioning, it serves as a well-suitable environment to achieve synchronicity on a software level. Nevertheless, since the direct image acquisition command evoking an action of included digital cameras is rather slow compared with the continuous readout mode of the camera live view, the latter is used in the following manner enabling acquisition rates up to $40 \mathrm{fps}$ (i.e. the maximum frame rate of the 
used UltraScan 1000XP camera): The camera live view is observed with a socalled image listener (DM terminology) and as soon as it is refreshed a scanning action is performed while the temporary image is copied to the memory.

Conceptually, there are two sources of intermediate delays causing possible signal integration in the subsequent image before the beam is moved to the desired position: Firstly, the data transfer and refreshing of the camera live view. Secondly, the execution time of the scanning action including software delays as well as the physical movement of the beam. The simplest empirical solution to guarantee signal integration at the aimed positions is the omission of sufficiently many images until the beam movement has terminated.

As the acquisition rate is lowered by skipping images and the necessity to do so is generally dependent on the application, four parameters can be set via the global tag settings of DM: The amount of skipped images (i) at the beginning of an acquisition (skip start), (ii) af-

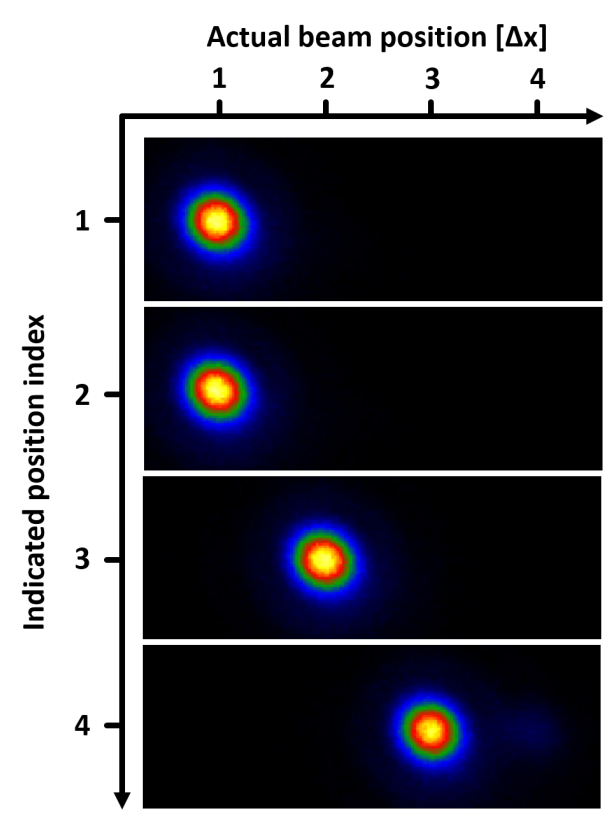

Figure 4.5: Indicated versus actual position of the direct probe for skip $=$ 0 , a pixel spacing $\Delta x=15 \mathrm{~nm}$, a probe size of approximately $8 \mathrm{~nm}$, and an exposure time of $10 \mathrm{~ms}$. ter each scanning line (skip flyback),

(iii) after a drift correction has been performed ( skip $_{\text {drift-correction), and (iv) }}$ between adjacent pixels (skip). Importantly, the speed loss due to (i)-(iii) is generally low as long as fewer or equally many lines than columns are scanned, which can be achieved without loss of generality by a $90^{\circ}$ scanning rotation. Consequently, these parameters have always been set to two guaranteeing accurate positioning even for long beam movements at speed losses of typically a few percent. On the contrary, omitting acquired images after each pixel, i.e. skip $>0$, reduces the scanning speed at least by a factor of two and should only be used if necessary. In order to visualise the consequences of a skip parameter too small for accurate positioning, the direct probe has been recorded with the camera while scanning yielding exactly the 


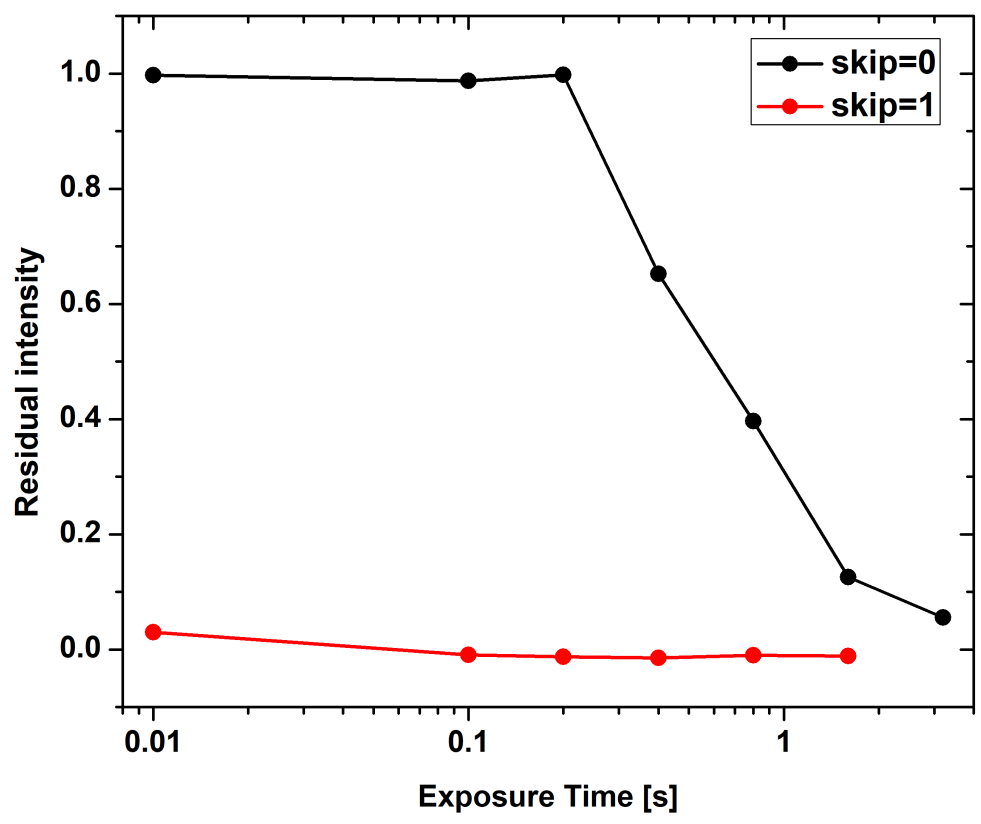

Figure 4.6: Dwell time dependence of the residual intensity $r$ at the former scanning position for skip $=0$ and skip $=1$ using a pixel size of $\Delta x=30 \mathrm{~nm}$ and no binning.

desired feedback, i.e. where the probe is physically located while the camera signal is integrated. The indicated versus actual beam position for skip $=0$, a pixel spacing $\Delta x=15 \mathrm{~nm}$, a probe size of approximately $8 \mathrm{~nm}$, and an exposure time of $10 \mathrm{~ms}$ is presented in Fig. 4.5 showing that the resulting signal is almost purely recorded at the beam position prior movement and

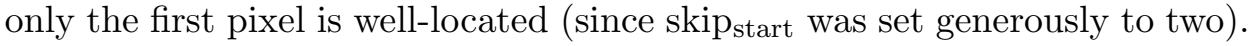
Generally, in various test acquisitions, significant signal was only observed at the former and the current indicated position but not in between indicating that the physical beam movement is much faster than reasonable 4D-STEM exposure times. This finding is consistent with typical sub-microsecond rise times of the involved scanning coils [133]. Consequently, the residual intensity $r=I_{\mathrm{A}} /\left(I_{\mathrm{A}}+I_{\mathrm{B}}\right)$ where $I_{\mathrm{A}}$ resp. $I_{\mathrm{B}}$ is the intensity (local maximum) at the former resp. current position serves as a quantitative measure of resulting inaccuracies and is close to one if all pixels (except for the first) 
are shifted to the left as shown in Fig. 4.5 and zero in the absence of positional inaccuracies. Importantly, for $r=1$, accurate positioning can be re-established by accounting for the predictable shift and adding an additional scanning column and only intermediate values deteriorate the spatial resolution in the acquired signal. The dwell time dependence of $r$ is shown for skip $=0$ and skip $=1$ in Fig. 4.6 revealing that the positional inaccuracies are generally small for exposure times on the second scale and skip $=1$ eliminates the finite values of $r$ down to $10 \mathrm{~ms}$ exposures. Please note that no camera binning has been used, which should maximise the effect of readout delays and that in case of indispensable accurate positioning $r$ should be measured with the desired camera settings. The data presented in this thesis was exclusively recorded at skip $=0$ as the used exposure time was either well below resp. above $1 \mathrm{~s}$,

After having discussed the synchronisation concept, the acquisition plugin's interface and capabilities shall be explained. The former is shown on the right in Fig. 4.7 and offers the following framework: Given a STEM reference image acquired with DigiScanII ("D: TestRef"), rectangular subregions for scanning and drift correction can be defined via regions of interest (ROIs) and subsequently assigned with the buttons "Assign ROI" resp. "Assign Drift". Furthermore, the running camera live view ("C: EF-CCD View") has to be assigned with "Assign Cam". In addition to a standard single-pass scan, the user can define the amount of images that shall be integrated per pass for each position ("Images per pass") as well as the number of passes ("Number of passes"). Besides the amount of pixels after which a drift correction should be performed, a maximum drift between two progressive corrections can be entered and the translation (restricted to the maximum drift range) yielding the smallest RMS difference between the former and current image will be corrected for. This approach aims for the suppression of erroneous translations while investigating crystalline materials with low drift rates but has yet to be assessed. All relevant results, i.e. the reference image, the data stack, the accumulated drift, and (if checked and thus acquired) a post acquisition reference image are saved in the indicated folder.

In principle, the simultaneous acquisition of classical detector signals, i.e. $\mathrm{BF} / \mathrm{ADF}$ resp. HAADF, is possible as well. Nevertheless, it is important to mention that obtaining the detector signal via scripting commands takes approximately $60 \mathrm{~ms}$ and might slow down the acquisition process significantly. Since virtual BF signals can be created from the data stack in most cases anyway, this option is currently not recommended as a default.

Lastly, the option of live-processing exists for all 4D-STEM applications 


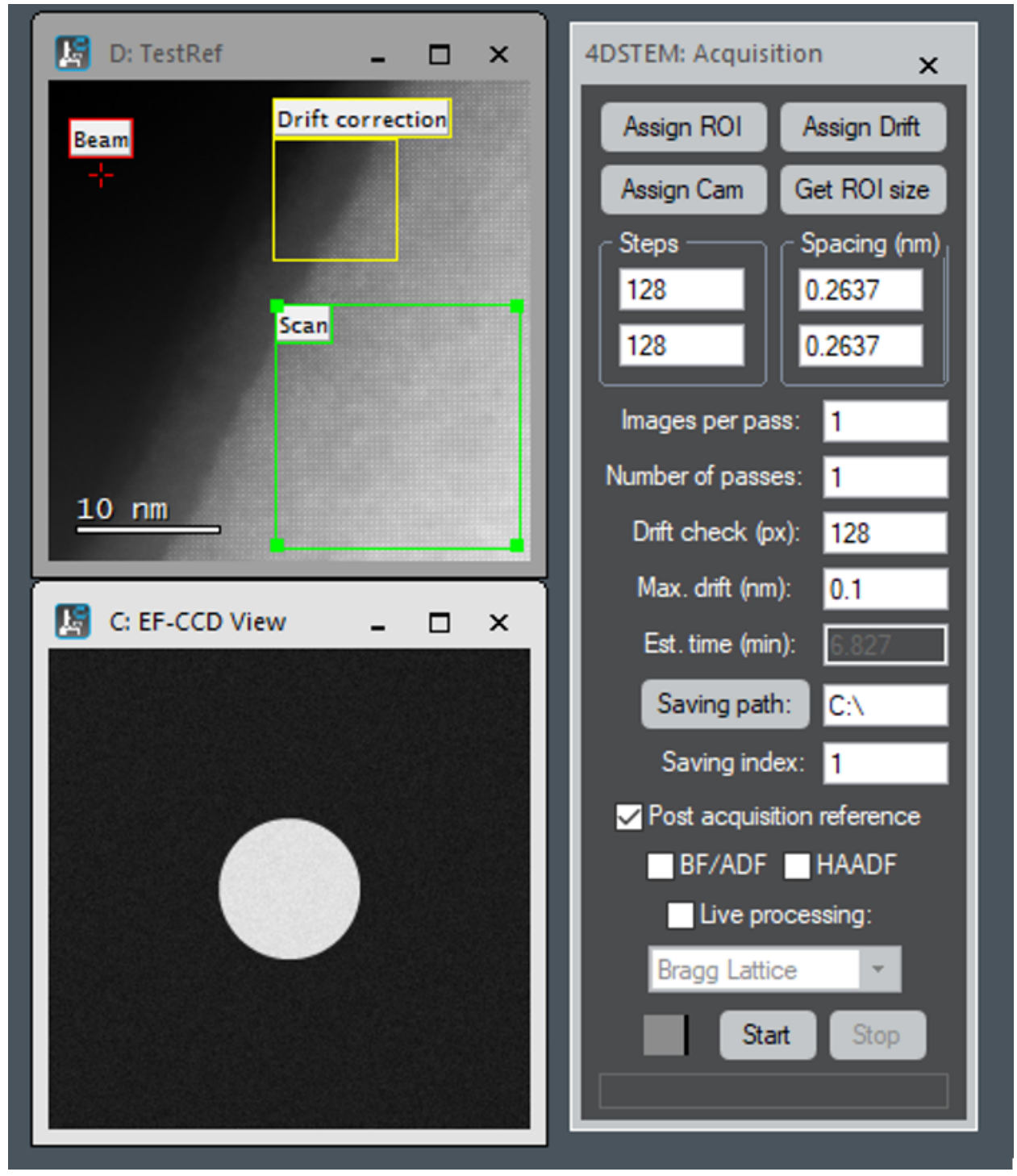

Figure 4.7: User interface of the 4D-STEM acquisition software (right) as well as a DigiScanII reference image (top left) and the camera live view (bottom left). The workflow for data acquisition and implementation details are given in the main text. 


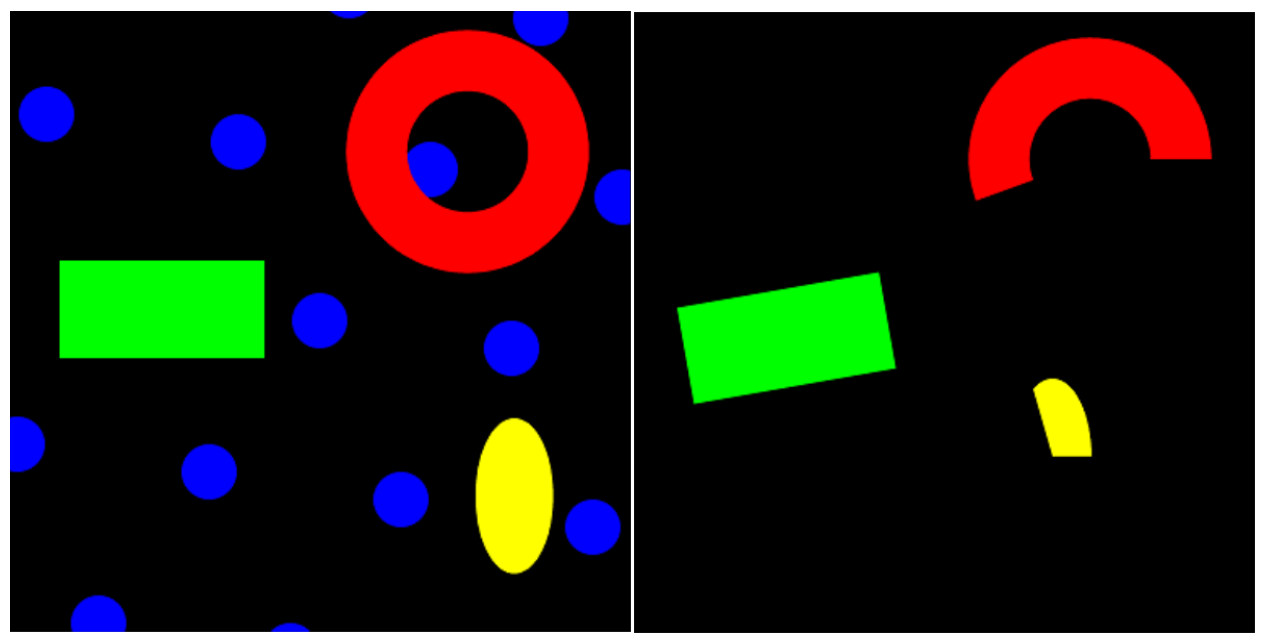

Figure 4.8: Exemplary mask shapes that can be created with the 4D-STEM analysis tool.

presented in the next subsection (except for fluctuation electron microscopy where intermediate user interaction is currently still needed). If activated, the recorded images are directly processed in the memory according to the frameworks shown below and subsequently deleted enabling for a larger sampling in real resp. reciprocal space, i.e. the acquisition of intermediate data well above the memory restrictions of the microscope computer. The complementary approach of saving the intermediate data directly to the computer's hard disk drive limits the acquisition rate tremendously and thus live-processing adds true value to existing strategies. Nevertheless, it should be noted that raw data is omitted this way and live-processing should only be used if the settings for the automatic data processing have been optimised priorly. Therefore, these parameters have to be set in the analysis tool while investigating an exemplary test acquisition obtained with the same experimental conditions and are subsequently automatically adopted by the acquisition software.

\subsubsection{D-STEM Analysis}

While the concept of 4D-STEM data acquisition explained in the previous subsection is rather general and mainly optical parameters of the condensor system need to be optimised for specific purposes, the subsequent numerical treatment of resulting signals depends largely on the application 4D-STEM 
is used for. Currently, the analysis software developed in this work includes virtual masking, the extraction of reciprocal lattice parameters for crystalline materials, phase contrast techniques, as well as fluctuation electron microscopy (FEM) tools. In order to demonstrate resulting applicabilities, examples of domain, strain and electric field mapping will be shown, as well as strategies for FEM data processing.

\section{Virtual Masking}
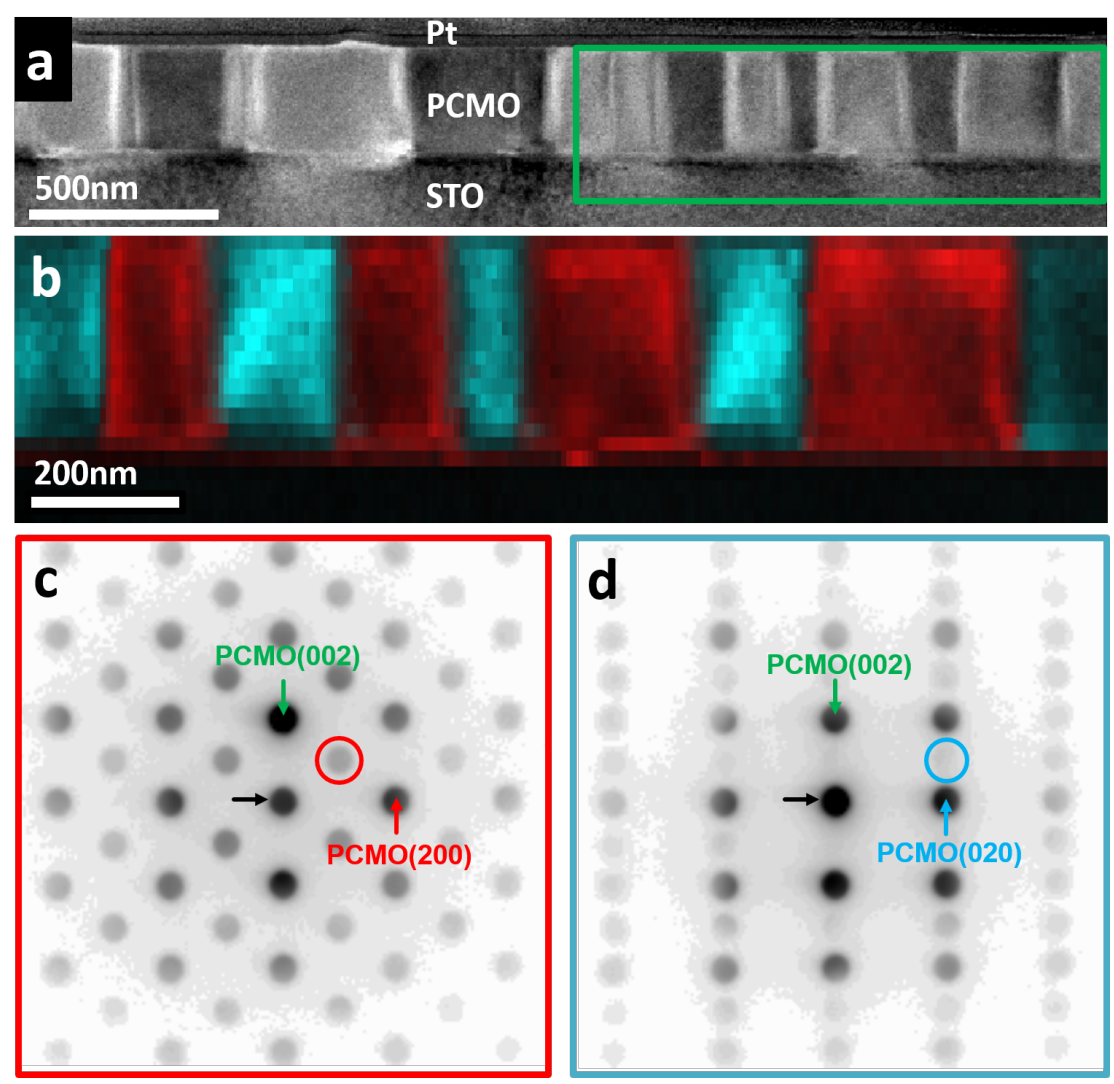

Figure 4.9: Domain mapping in a PCMO thin film: Two different crystal orientations are found in the scanning area in (a) marked with the green rectangle. Filtering the intensity in corresponding unique Bragg reflections marked with the red resp. cyan circles in (c) resp. (d) yields the false colour map in (b). The figure is reprinted from [134] with permission.

The main challenge in virtual masking, i.e. the creation of virtual detectors 
in the signal space, is the implementation of a user-friendly interface enabling for an easy definition of various detector shapes. Here, by combining native DM tools with custom operations, the exemplary mask shapes presented in Fig. 4.8 can be created graphically. Furthermore, employing the loop tool of DM, arbitrarily shaped closed masks can be defined as well. In addition, in order to allow for the superposition of different signal domains, four different colours, i.e. red, green, blue and yellow, can be assigned and mixed with variable weighting factors with the remaining voids. The user interface itself as well as the intended workflow is illustrated in Fig. A.2. An example of how circular masks can be used for the sake of domain mapping in a PCMO thin film is given in Fig. 4.9 and its interpretation will be described in detail in section 6.2. For now, it is only important that two different crystal orientations with corresponding diffraction patterns (c) and (d) are found in the scanning area marked with the green rectangle in (a). Filtering the intensity inside the red (c) resp. cyan (d) circles corresponding to unique Bragg reflections of the respective orientations yields the false colour map shown in (b). While this example qualitatively visualises material heterogeneities as it was originally suggested in [106, 135], quantitative virtual masking approaches of 4D-STEM data were reported as well, e.g. in the context of light element localisation [136].

\section{Bragg Analysis: Strain Mapping of Crystalline Materials}

The accessibility of spatially resolved diffraction patterns, i.e. nano-beam electron diffraction (NBED), enables for extraction of local reciprocal lattice constants of crystalline materials and thus the observation of their strain state. Initially, this strategy was suggested in [137] and the resulting precision subsequently compared to other TEM techniques in [138] and [139] by considering the standard deviation of the measured strain in presumably unstrained sample regions. Typical values for NBED were quantified to approximately $1 \times 10^{-3}$ being a few times higher than standard deviations obtained by convergent beam electron diffraction (CBED) and off-axis electron holography. Dynamic diffraction and inelastic scattering were found to be the main limitations impeding a precise localisation of diffracted discs and numerical approaches to suppress these effects were suggest in [130, 131]. Complementary, as mentioned previously, structured apertures can be used enabling for more accurate disc detection via auto and cross correlations of resulting diffraction patterns [128]. The analysis of the latter correlation functions is included in the presented 4D-STEM analysis tool and a step-by-step documentation how the Bragg spots are extracted after auto or 
cross correlation is given in Fig. A.3. Subsequently, a two-dimensional lattice is fitted to the extracted positions via the following linear optimisation problem:

$$
\min _{\vec{b}_{1}, \vec{b}_{2}} \sum_{(i, j) \in \mathfrak{I}} I(i, j)^{2}\left(\vec{k}(i, j)-i \vec{b}_{1}-j \vec{b}_{2}\right)^{2} .
$$

Here, $I(i, j)$ denotes the intensity in the auto or cross correlation of the extracted disc of order $(i, j)$ located at $\vec{k}(i, j)$ and $\mathfrak{I}$ the index set of all included reflections. $\vec{b}_{1}$ and $\vec{b}_{2}$ are identified as local reciprocal lattice vectors.

In order to demonstrate the applicability of both the structured aperture described previously as well as the numerical implementation in the context of strain mapping, a silicongermanium test sample, grown along the [001] direction with molecular beam epitaxy (MBE) by Y. Barnscheidt (Leibniz University Hannover), shall be analyzed. Fig. 4.10 (a) shows an HAADF STEM overview of the grown structure including two approximately $10 \mathrm{~nm}$ thick $\mathrm{Si}_{1-x} \mathrm{Ge}_{x}$ layers with nominal compositions $x=0.28$ (bottom) resp. $x=0.42$ (top) inside a silicon matrix. The resulting diffraction pattern of the silicon substrate in [110] orientation using the $3 \mathrm{mrad}$ bullseye aperture is given in Fig. 4.10 (b). For the sake of simplicity, the local

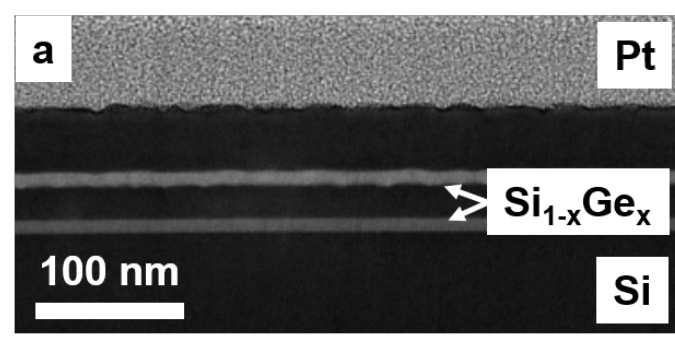

b

\section{$4 \mathrm{~nm}^{-1}$}

Figure 4.10: (a) HAADF STEM overview of the $\mathrm{Si}_{-} \mathrm{Si}_{1-x} \mathrm{Ge}_{x}$ layered sample with (b) corresponding diffraction pattern using the $3 \mathrm{mrad}$ bullseye aperture. lattice parameters $a$ (in-plane) resp. $c$ (out-of-plane) - extracted from their reciprocal counterparts - will be compared to the silicon substrate by considering their relative deviations $\left(a-a_{\mathrm{Si}}\right) / a_{\mathrm{Si}}$ resp. $\left(c-c_{\mathrm{Si}}\right) / c_{\mathrm{Si}}$. A line profile of the latter in combination with the atomic Ge concentration quantified via EDX using the Cliff-Lorimer method, i.e. Eq. (3.2) and $k_{\mathrm{Ge}} / k_{\mathrm{Si}}=1.65$, is shown in Fig. 4.11 revealing that the experimentally found Ge content is slightly below the nominal 


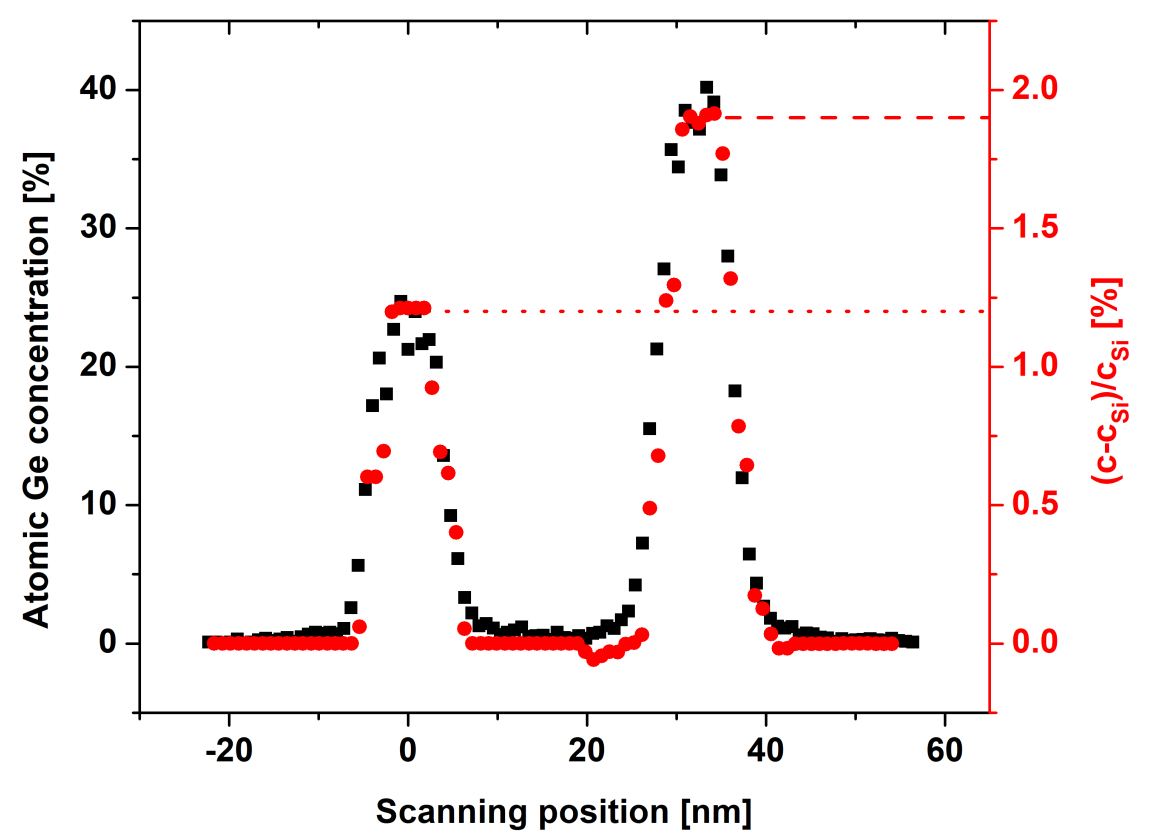

Figure 4.11: Germanium concentration $x$ quantified by EDX (black) as well as the relative out-of-plane lattice parameter deviations $\left(c-c_{\mathrm{Si}}\right) / c_{\mathrm{Si}}$ (red) across the $\mathrm{Si}_{1-x} \mathrm{Ge}_{x}$ layers shown in Fig. 4.10. The dashed and dotted lines correspond to the experimental results of the latter given in [140] for $x=0.3$ and linearly scaled to $x=0.38$ resp. $x=0.24$.

values. In addition, the dashed resp. dotted red lines indicate the expected values for $\left(c-c_{\mathrm{Si}}\right) / c_{\mathrm{Si}}$ based on the experimental results in [140], i.e. $\left(c-c_{\mathrm{Si}}\right) / c_{\mathrm{Si}}=1.5 \%$ for $x=0.3$ in Fig. 1 , assuming $x=0.24$ resp. $x=0.38$ and a linear scaling of lattice parameters with the Ge content. Generally, the shape of the profiles is similar and the dashed and dotted lines agree well with measured values of $\left(c-c_{\mathrm{Si}}\right) / c_{\mathrm{Si}}$. Nevertheless, comparing the results with the analytical expression for coherent growth of cubic materials [141], i.e.

$$
\frac{c-c_{\mathrm{Si}}}{c_{\mathrm{Si}}}=0.041 \cdot x \cdot \frac{1+\nu}{1-\nu},
$$

yields significantly different values of $1.74 \%$ resp. $2.76 \%$ using Poisson's 
ratio $\nu=0.28$ for $\mathrm{Si}[001]$ [142]. As the latter varies only slightly with the Ge content [142], the discrepancy is most likely caused by the simplifying assumptions on which Eq. (4.2) is based, i.e. the neglection of thin foil effects as well as partial relaxation. In fact, the negative values of $\left(c-c_{\mathrm{Si}}\right) / c_{\mathrm{Si}}$ at the tails of the right peak in Fig. 4.11 are very characteristic for thin foil strain states [141, 143] indicating that the first assumption is not met. The consistency of the peak heights with the results in [140] obtained by off-axis holography and at similar thicknesses suggests that the deviations from Eq. (4.2) are not caused by the 4D-STEM analysis. The lamella thickness was determined to approximately $75 \mathrm{~nm}$ via Eq. (3.1) with $\lambda=194 \mathrm{~nm}$ [144]. In order to both assess the layers' homogeneity as well as demonstrate the potential of live-processing, a
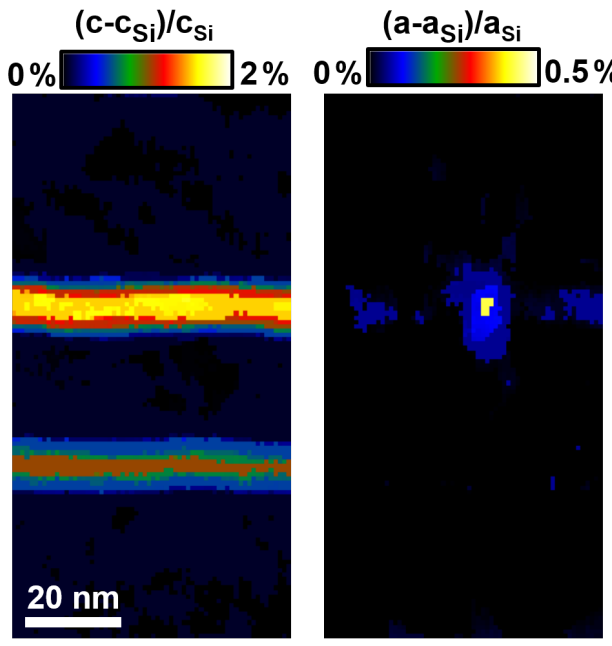

Figure 4.12: Large field of view strain maps taken with live-processing revealing heterogeneities in the $\mathrm{Si}_{1-x} \mathrm{Ge}_{x}$ layer with $x=0.38$. larger field of view was scanned with the same parameters for reciprocal lattice vectors extraction resulting in the maps of $\left(a-a_{\mathrm{Si}}\right) / a_{\mathrm{Si}}$ (right) and $\left(c-c_{\mathrm{Si}}\right) / c_{\mathrm{Si}}$ (left) shown in Fig. 4.12. While for $x=0.24$ no significant changes of the in-plane lattice parameter is observed, heterogeneities exist for $x=0.38$. The line profile shown previously in Fig. 4.11 was taken in the left part of Fig. 4.12 where the in-plane lattice constant exhibits little variations being consistent with the linear dependence between $\left(c-c_{\mathrm{Si}}\right) / c_{\mathrm{Si}}$ and $x$. The subtle influence on the out-of-plane lattice constant suggest that the strain relaxation via these heterogeneities is rather small. Noteworthy, the shown maps are the result of 100 GB live-processed data. The standard deviation of the strain in the substrate amounts to $1.2 \times 10^{-4}$, which is consistent with the results in [128]. Still, as discussed thoroughly in the cited reference, the standard deviation depends on experimental parameters such as the counts and pixel spacing in the diffraction patterns or the sample thickness hence an acquisition of a presumably unstrained internal reference recorded with the given conditions is always desirable when performing strain measurements. In the given ex- 
ample, a mean value of 94 counts/px and a pixel spacing of $0.02 \mathrm{~nm}^{-1}$ was used.

\section{Phase Contrast: Electric Field Mapping}

As already mentioned in chapter 3 , the phase of the electron exit wave contains valuable information about the potential it has interacted with. Consequently, phase contrast techniques are of high relevance in the context of 4D-STEM and for instance applied to visualise electric and magnetic fields [104, 145]. One of the biggest obstacles to extract the latter in matter is - just as in complementary techniques like off-axis electron holography - the disentanglement from contrasts caused by the inner potential of the investigated sample $[146,147]$. Thus, in the case of crystalline material, it is desirable to find a sample orientation in which diffraction contrasts are strongly suppressed [148]. Please note that effects caused by the mean inner potential still remain if the crystal is tilted out of zone axis.

As summarised and compared in [105], different approaches can be used to extract phase information from the intensity distribution of the transmitted electron beam: Firstly, by calculating its first statistical moment. Secondly, by defining a virtual segmented detector with four-fold symmetry and considering intensity differences inside the respective segments - also referred to as virtual differential phase contrast (DPC). Thirdly, by tracing the edge of the direct beam via Sobel filtering and cross correlation with a given reference - also referred to as edge detection. All three strategies are implemented in the 4D-STEM analysis tool and described in detail in Fig. A.4, A.5, and A.6. The second approach is the virtual counterpart to segmented DPC detectors [149] and serves rather as a comparison of this possibly direct implementation with first moment and edge detection phase contrast making use of the full reciprocal information contained in the diffraction patterns. In fact, the phase contrast transfer functions differ among the techniques as DPC projects all intensity redistributions to the geometrical centers of the detector segments $[150,151]$ and edge detection assumes an unchanged shape of the direct beam, which is only satisfied if the phase varies solely linearly on the scale of the incident probe [105].

In order to demonstrate the implementation in the herein presented software and compare the different approaches, Fig. 4.13 shows the resulting signals of two opposed tungsten tips (cropped in black) with externally applied voltage of $15 \mathrm{~V}$. The experiment was performed by U. Ross and J. Lindner using a semi-convergence angle of $120 \mu \mathrm{rad}$, a mean value of 80 counts/px inside the direct disc, a pixel spacing of $1.89 \mu \mathrm{rad}$, and a tip spacing of ap- 


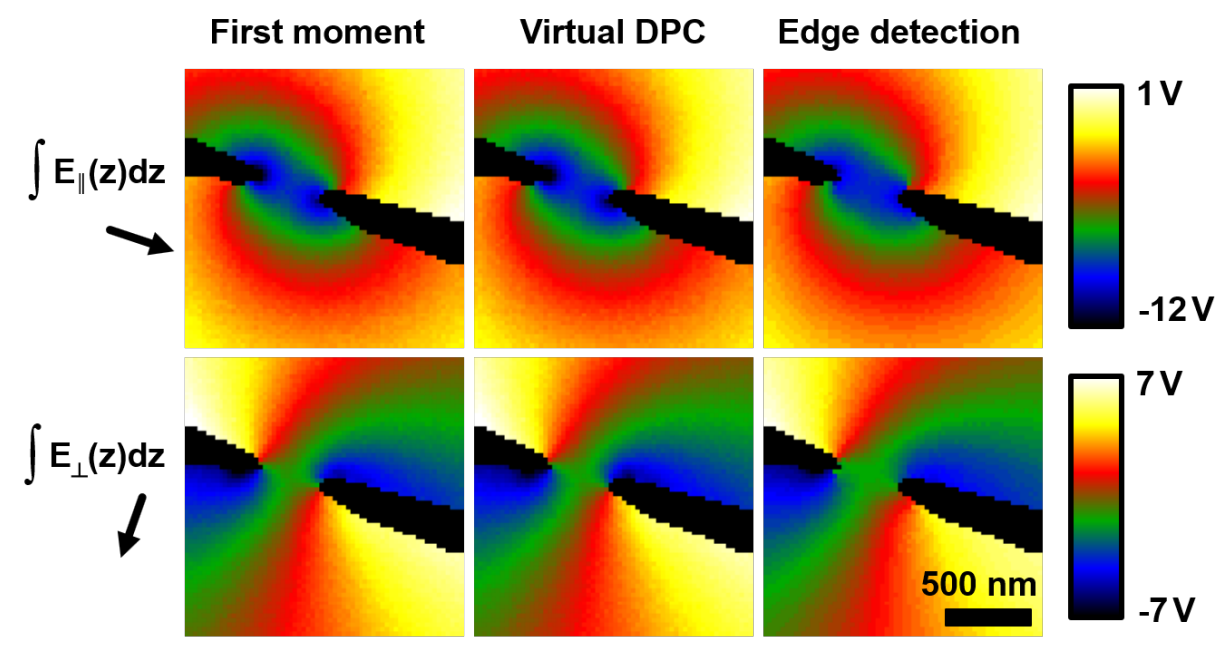

Figure 4.13: Phase contrast signals of two opposed tungsten tips with an externally applied voltage of $15 \mathrm{~V}$ obtained by first moment (left), virtual DPC (center), and edge detection (right): While the former two can directly be interpreted as projected electric field along (top) resp. perpendicular (bottom) to the tip connection line, the latter shows deviations close to the tips where the gradient of the field gets large.

proximately $400 \mathrm{~nm}$. Resulting deflection angles have been translated to the unit of integrated electric fields, i.e. volts, via division by the reduced interaction constant [152]

$$
C^{\prime}=\frac{m_{0} c^{2}+e U}{U\left(2 m_{0} c^{2}+e U\right)}
$$

Here, $e$ is the elementary charge, $m_{0}$ the electron rest mass, $c$ the velocity of light, and $U$ the acceleration voltage of the microscope. For $U=300 \mathrm{kV}$, Eq. (4.3) yields $C^{\prime}=2.04 \mu \mathrm{rad} / \mathrm{V}$. The in-plane coordinates are chosen along (top) and perpendicular (bottom) to the tip connection line and all values are given relative to the upper right corner. While first moment and virtual DPC yield virtually identical results, i.e. the integrated electric field as there is no sample interaction [152], the average beam deflection close to the tips along their connection line is slightly underestimated in the case of edge detection. This finding can be explained by the increasing heterogeneity of the field, i.e. the curvature of the phase, leading to distortions of the direct diffraction disc. Furthermore, the strongly broken inversion symmetry of 
the respective directions suggests either a difference in height or position of the tips along the optical axis and reflects nicely the necessity of threedimensional modelling for quantitative data interpretation [152].

\section{Fluctuation Electron Microscopy}

The experimental observation of medium-range order effects in amorphous materials by means of fluctuation electron microscopy (FEM), i.e. a statistical analysis of short-range order diffraction intensities, has been demonstrated in $[153,154]$. While initially pursued in the TEM mode, FEM was in accordance with the reciprocity theorem [155] transferred to STEM [103] where position dependent diffraction patterns are taken with different illumination conditions. Consequently, FEM belongs to the family of 4D-STEM techniques and is included in the presented analysis tool. As summarised in [156], different strategies can be used to calculate $k$-resolved normalised variances $V(k)$ from the resulting diffraction intensities $I\left(x, y, k_{x}, k_{y}\right)$ at the real and reciprocal space coordinates $(x, y)$ resp. $\left(k_{x}, k_{y}\right)$ :

1. Variance of annular means:

$$
V(k)=\frac{\left\langle\left\langle I\left(x, y, k_{x}, k_{y}\right)\right\rangle_{k}^{2}\right\rangle_{r}}{\left\langle\left\langle I\left(x, y, k_{x}, k_{y}\right)\right\rangle_{k}\right\rangle_{r}^{2}}-1 .
$$

2. Mean of ring variances:

$$
V(k)=\left\langle\frac{\left\langle I^{2}\left(x, y, k_{x}, k_{y}\right)\right\rangle_{k}}{\left\langle I\left(x, y, k_{x}, k_{y}\right)\right\rangle_{k}^{2}}\right\rangle_{r}-1 .
$$

3. Ensemble variance:

$$
V(k)=\frac{\left\langle\left\langle I^{2}\left(x, y, k_{x}, k_{y}\right)\right\rangle_{k}\right\rangle_{r}}{\left\langle\left\langle I\left(x, y, k_{x}, k_{y}\right)\right\rangle_{k}\right\rangle_{r}^{2}}-1 .
$$

4. Annular mean of variance image:

$$
V(k)=\left\langle\frac{\left\langle I^{2}\left(x, y, k_{x}, k_{y}\right)\right\rangle_{r}}{\left\langle I\left(x, y, k_{x}, k_{y}\right)\right\rangle_{r}^{2}}\right\rangle_{k}-1 .
$$

Here, $\langle\cdot\rangle_{r}$ and $\langle\cdot\rangle_{k}$ denote averaging operators over all scanning positions resp. the domain $\left\{\left(k_{x}, k_{y}\right): k^{2} \leq k_{x}^{2}+k_{y}^{2}<(k+\delta k)^{2}\right\}$ for a fixed finite resolution $\delta k$ in the normalised variance curves. 
While the calculation according to the given formulas is straight forward, a major experimental challenge is the proper alignment of the individual diffraction patterns (DPs), i.e. the localisation of their reciprocal origin, since the intense direct beam is typically hidden behind a beam stop. This task can be split in three processes: Firstly, correcting for scanning position dependent shifts of the DPs with respect to the beam stop. Secondly, correcting for shifts of the DPs including the beam stop. Thirdly, finding the global origin after having corrected for the position dependent shifts. Please note that this definition does not include any assumption about the origins of the shifts in the first place. Still, it is experimentally motivated as the position dependent shifts are typically attributed to residual beam tilts caused by the scanning process resp. instabilities in the projection system observed in the herein used Titan and the latter identification as a shift influencing the DPs and the beam stop likewise is presuming. However, the shared architecture of all functions included in the presented analysis tool allows for flexible combination of different routines as shown in the step-bystep FEM data preparation in Fig. A.7 making use of e.g. the phase contrast techniques presented above: Besides the correction of the scanning induced beam tilt, position changes of the beam stop can be extracted as the translation yielding the minimal RMS difference of image subregions. This feature is exemplary visualised in Fig. 4.14 showing (a) an averaged

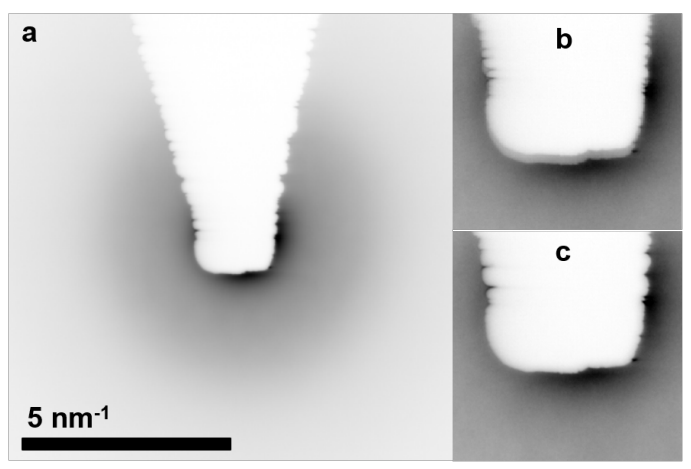

Figure 4.14: (a) Averaged NBED pattern of a silicon-rich silicon oxide as well as zooms of the central region (b) before and (c) after correction for apparent movements of the beam stop. NBED pattern of a silicon-rich silicon oxide as well as enlargements of the central region (b) before and (c) after correction for the apparent beam stop movement. In fact, as shown in Fig. 4.15, this correction has an impact on the subsequent FEM analysis and leads to lower normalised variances in Eq. (4.4) as domains with low and high diffraction intensities are mixed prior correction. Generally, the significance of the processing steps shown in Fig. A.7 depends on the alignment of the scanning coils, i.e. the magnitude of scanning induced tilts, as well as the stability of the used microscope. However, changes in the peak heights 


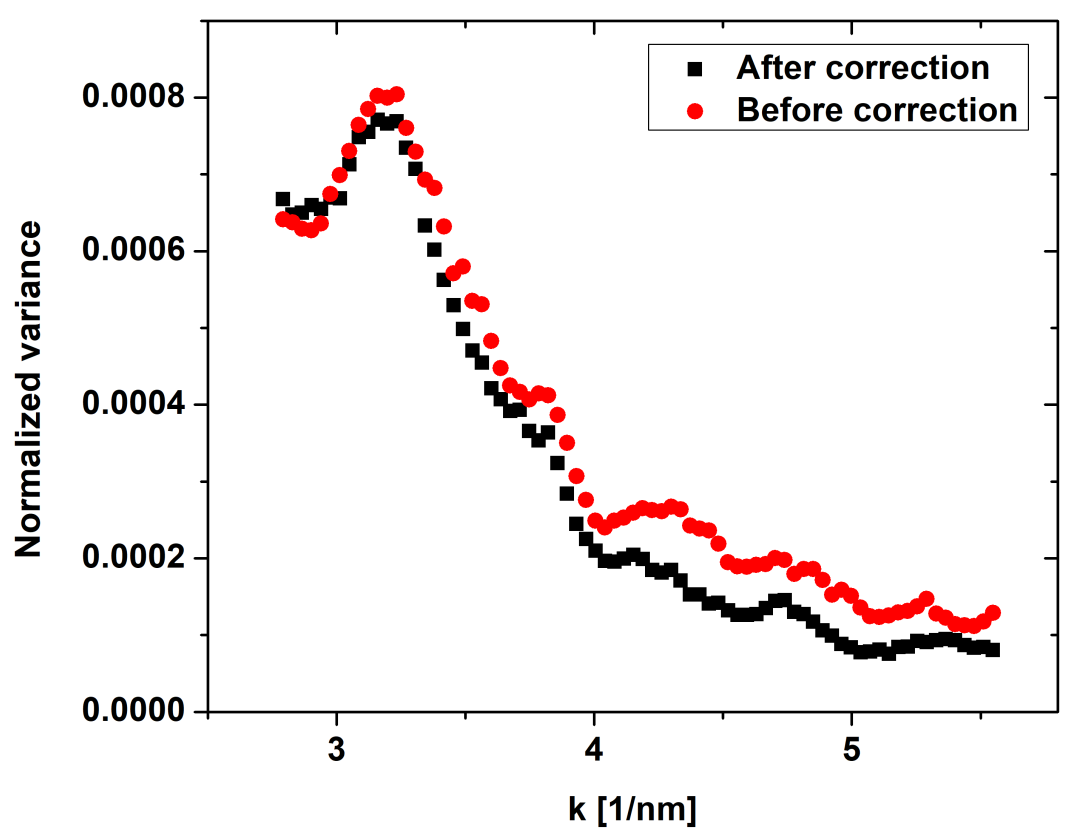

Figure 4.15: Normalised variance profiles following Eq. (4.4) of the FEM stack presented in Fig. 4.14 before (red) and after (black) correction for apparent beam stop movements.

as shown in Fig. 4.15 can be crucial in the context of FEM as they are quantitatively used in subsequent analysis steps, e.g. when observing their dependence of the used probe size to infer information about medium-range order as it is done in variable resolution FEM [103].

\section{Included Libraries and Benchmarking}

Lastly in the context of the developed 4D-STEM analysis tool, included libraries will be listed and a benchmarking of the data throughput in the respective applications will be given. While the presented user interfaces are exclusively created in the native DM scripting language, extensive calculations are handed over to self-written $\mathrm{C}++$ routines using CPU parallel computing. The routines are based on the DM software development kit [116] and include Intel ${ }^{\circledR}$ Threading Building Blocks 2.2 for CPU parallelism 


\begin{tabular}{cc} 
Application & Throughput $[\mathrm{GiB} / \mathrm{s}]$ \\
\hline Virtual Masking & $4.3(2)$ \\
Bragg Analysis & $0.19(1)$ \\
Edge detection & $0.17(1)$ \\
First moment & $1.67(3)$ \\
Virtual DPC & $1.91(7)$ \\
FEM & $0.17(1)$ \\
\hline
\end{tabular}

Table 4.1: Throughput while analyzing a stack containing 4096 diffraction patterns with 256 by 256 pixels (signed 4-byte integers) resulting in a stack size of $1 \mathrm{~GB}$. The analysis was performed using an AMD FX-6300 with three physical CPU cores and 8 GB DDR3 RAM.

[157], FFTW 3.3.5 for fast discrete Fourier transformations [158], and Armadillo 3.930.1 for convenient matrix operations [159]. The resulting data throughput of the above mentioned applications are summarised in Tab. 4.1 .

\subsection{Synchronisation and Quantification of STEM Signals}

The acquisition of 4D-STEM data described in the previous section was realised in a software environment controlling both the beam position as well as the detector read-out simultaneously. Generally, such an environment does not exist for an arbitrary signal demanding for further synchronisation strategies if non-classical STEM signals, e.g. STEM-EBIC, shall be recorded. In the context of this thesis, three different approaches have been pursued: (i) software-synchronisation via a shared network folder, (ii) hardware-synchronisation using an integrated analogue digital converter (ADC) that is triggered by the scanning unit, and (iii) a posteriori synchronisation by moving the beam to a marker position resulting in a unique signal at the end of every scanning line.

The first approach was implemented in collaboration with J. Lindner to record the STEM-EBIC signals presented later on in section 7.2 as follows: A LabView-controlled computer digitises the output voltage of the current amplifier, i.e. the signal in question, with a fixed frequency. After starting the corresponding LabView routine, a text file is created in a shared network folder. Simultaneously, a DM script observes the folder and starts the scanning routine as soon as the file is found. Obviously, there is a finite delay 
between the respective actions, which can be estimated to several tens of milliseconds by running test scripts creating several alternating trigger files. Unfortunately, the delay cannot be predicted precisely as it fluctuates on the same order. Consequently, the dwell time has to be kept well above these fluctuations to maintain a well-defined mapping between scanning positions and collected signals slowing the acquisition process down. Nevertheless, due to its rather simple implementation, the approach is well-suitable for signals with long integration times or for proving the concept of novel STEM signal collection.

The second approach, in contrast to the others, involves hardware-interactions, i.e. a trigger created by the scanning unit of the microscope evoking the digitisation in the ADC of the Gatan DigiScanII unit installed at the FEI Titan. Normally, the latter is used to convert the output voltage of standard STEM detectors hence by simply exchanging the input cable a full integration in DM can be achieved enabling for synchronous acquisitions with microsecond dwell times as well as simultaneous collection of further classical signals. In order to quantify the resulting digital values, which are internally translated to electron counts, the ADC needs to be calibrated. The voltage and dwell time dependence of the indicated counts is shown in Fig. A.8 and A.9. Even though this approach is much easier realised than software- and a posteriori synchronisation, it is worth mentioning that it is in contrast to the others limited to voltage signals and an expansion to e.g. higher-dimensional data demands for more sophisticated trigger in- and outputs.

Lastly, an approach for a posteriori synchronisation, i.e. the correlation of independent scanning and acquisition processes, has been implemented in the context of this thesis and published in the following manuscript [160] in Ultramicroscopy (doi.org/10.1016/j.ultramic.2019.02.020) - the author's contributions are given in the main text and the supplementary material is included in section A.10. All contents are reprinted in their original form with permission.

By moving the electron beam to a marker position with a unique signal after each scanning line, resulting uncertainties in the spatially reconstructed signals were quantified to be approximately half the pixel spacing. Compared with the two other strategies, a posteriori synchronisation demands on the one hand side for the presence of a unique marker position (or more generally a marker sequence). On the other hand, it is more flexible as neither hardware- nor software-communication is needed resulting in a gain in acquisition speed since intermediate waiting periods are eliminated. 


\title{
A posteriori synchronization of scanning transmission electron microscopy signals with kilopixel per second acquisition rates
}

\author{
Tobias Meyer, $^{*,}$, Sergei Lopatin ${ }^{\mathrm{b}}$, Michael Seibt $^{\mathrm{a}}$, Vladimir Roddatis $^{\mathrm{c}}$ \\ ${ }^{a}$ 4th Institute of Physics - Solids and Nanostructures, University of Goettingen, Friedrich-Hund-Platz 1, Göttingen 37077, Germany \\ ${ }^{\mathrm{b}}$ King Abdullah University of Science and Technology (KAUST), Core Labs, Thuwal, 23955-6900, Saudi Arabia \\ ${ }^{\mathrm{c}}$ Institute of Materials Physics, University of Goettingen, Friedrich-Hund-Platz 1, Göttingen 37077, Germany
}

\section{A R T I C L E I N F O}

\section{Keywords:}

STEM

Synchronization

EELS

EDX

4DSTEM

\begin{abstract}
A B S T R A C T
The stability and sensitivity of scanning transmission electron microscopes as well as detectors collecting e.g. electrons which suffered different scattering processes, or secondary radiation, have increased tremendously during the last decade. In order to fully exploit capabilities of simultaneously recording various signals with up to $1000 \mathrm{px} / \mathrm{s}$ acquisition rates the central issue is their synchronization. The latter is frequently a non-trivial problem without commercially available solution especially if detectors of different manufacturers are involved. In this paper, we present a simple scanning pattern enabling a posteriori synchronization of arbitrarily many signals being recorded entirely independently. We apply the approach to the simultaneous atomic-scale acquisition of signals from an annular dark-field detector and electron energy loss as well as energy-dispersive x-ray spectrometers. Errors emerging in scanning direction due to the independence of the respective processes are quantified and found to have a standard deviation of roughly half the pixel spacing. Since there are no intermediate waiting periods to maintain synchronicity, the proposed acquisition process is, in fact, demonstrated to be $12 \%$ faster than a commercial hardware-synchronized solution for identical sub-millisecond signal integration times and hence follows the trend in electron microscopy to extract more information per irradiating electron.
\end{abstract}

\section{Introduction}

Scanning transmission electron microscopy (STEM) offers a vast variety of signals which can be recorded simultaneously [1]. Besides the detection of electrons being scattered in a certain angular range, analytical methods like electron energy-loss spectroscopy (EELS) and energy-dispersive X-ray spectroscopy (EDX) [2,3] are commonly used in order to determine the chemical composition of materials. However, synchronizing the acquisition of signals with the scanning movement of the focused electron beam can be challenging, especially since recent progress in detection and lens systems technology, enabling high-speed data collection with sub ̊̊ spatial resolution [4-6], demands for synchronicity at kilopixel per second scanning speeds.

In this work, we introduce an experimental scheme which completely separates data acquisition and synchronization. More explicitly, data sets from various sources are recorded independently, i.e. without any hardware synchronization. This type of data acquisition will subsequently be referred to as 'streaming mode'. For a posteriori synchronization, marker signals are included into the acquisition at specific previously defined points. As an example, we use a script as described in the following section to move the beam to a position in vacuum after each completed scanning line. Subsequently, the beginning and end of each scanning line can be identified in the acquired data by the marker enabling for a spatial reconstruction.

The concept is shown to be applicable to kilopixel per second data collection. Since there are no waiting periods to maintain hardware synchronicity during the acquisition, faster data collection can be realized, i.e. a ratio of acquisition time and life time close to one.

Although the quality of a posteriori synchronized data is comparable to that of traditionally recorded data, some inherent error sources can be identified as being due to the missing phase lock, different clocking periods as well as clocking irregularities of different recording devices. These errors are described quantitatively and confirmed by a comparison to images resulting from hardware-synchronized data.

\section{Experimental procedure}

\subsection{Sample preparation and microscopy parameters}

An electron transparent $\mathrm{SrTiO}_{3}$ (STO) foil has been extracted from a

\footnotetext{
* Corresponding author.

E-mail address: tmeyer@uni-goettingen.de (T. Meyer).
} 
commercial substrate employing an FEI Nova NanoLab Dual Beam focused ion beam. During mounting on the support grid, the stage was tilted to $20^{\circ}$ guaranteeing a coinciding decrease of X-ray shielding with the zone axis alignment in the STEM. All data has been collected in an FEI Titan 80-300 operated at $300 \mathrm{kV}$ and equipped with a Gatan Quantum 965 ER image filter as well as a stand-alone Oxford Instruments X-Max $80 \mathrm{~mm}^{2}$ EDX detector. The energy ranges for the chemical analysis of EELS and EDX data are summarized in Table 1. Additionally, a Keithley DMM6500 digital multimeter has been used to continuously read out the output voltage of the Gatan annular dark field (ADF) detector included in the image filter which is proportional to the electron current impinging on the detector. The inner and outer diameter of the detector are $4.3 \mathrm{~mm}$ and $24 \mathrm{~mm}$, respectively. Throughout all experiments the beam current was set to $100 \mathrm{pA}$ and the camera length was chosen to be $38 \mathrm{~mm}$ resulting in an acceptance semiangle of the spectrometer of $39 \mathrm{mrad}$ and an inner and outer collection semi-angle of the ADF detector of $46.8 \mathrm{mrad}$ and $200 \mathrm{mrad}$.

\subsection{Data acquisition and reconstruction}

The beam movements in streaming mode have been controlled by a custom Gatan Digital Micrograph (DM) script employing a Gatan DigiScan II scan unit. Image reconstructions were performed using custom DM scripts as well. All scripts are available in the supplementary material [7]. A sketch of the post-acquisition reconstruction procedure is given in Fig. 1: After the marker signal is localized in the continuous readout of the detector (a) the lines are arranged (b). Due to irregularities in the acquisition or scanning speed the length of the lines might differ slightly hence they are scaled (c) and interpolated (d) to the desired amount of scanning positions per line.

\section{Results and discussion}

In this section, two exemplary combinations of signals will be elaborated. Firstly, ADF as well as low-loss EELS signals collected with $1000 \mathrm{px} / \mathrm{s}$ acquisition rates will be compared to prove that the suggested scanning pattern is applicable to high-speed data collection and to quantify resulting errors. Secondly, a lower acquisition rate of $10 \mathrm{px} /$ $\mathrm{s}$ is chosen to include the possibility of EDX spectra collection in order to emphasize the high methodology's versatility.

\subsection{High-Speed data acquisition}

Fig. 2(a) shows an ADF overview image (acquired in standard STEM mode) of [100] oriented STO including the scanned region of interest (ROI) as well as the vacuum marker position of the first example. The total acquisition time for 64 by 128 pixels inside the ROI and an acquisition rate of $1000 \mathrm{px} / \mathrm{s}$ was $8.3 \mathrm{~s}$. Additionally, excerpts of the ADF detector output voltage and the integrated EELS spectra are given in (b) and (c), respectively, in order to illustrate typical signals obtained in streaming mode. Note that the entrance aperture of the spectrometer

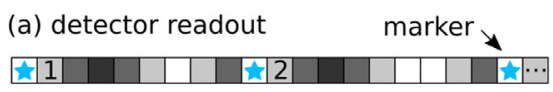

(b) arranged

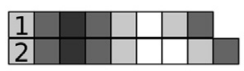

(d) interpolated

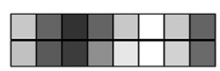

Fig. 1. Sketch of the image reconstruction. The continuous readout of the detector (a) is, according to the marker positions, arranged line by line (b) and subsequently scaled (c) and interpolated (d) to the amount of desired scanning positions.
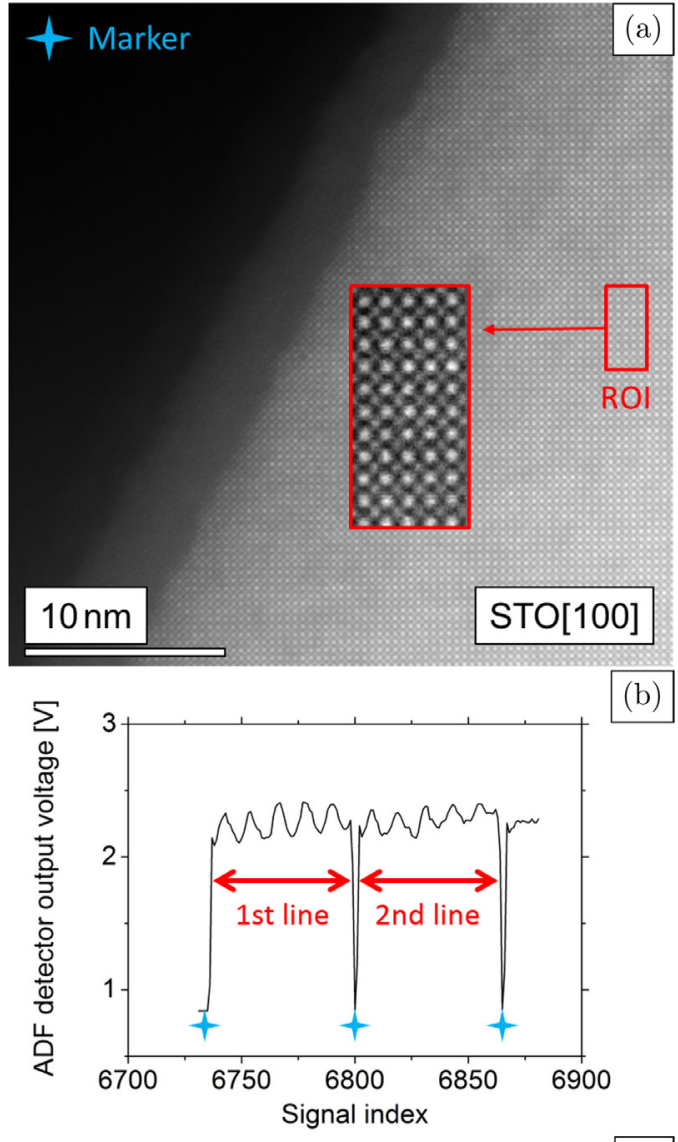

$(\mathrm{c})$

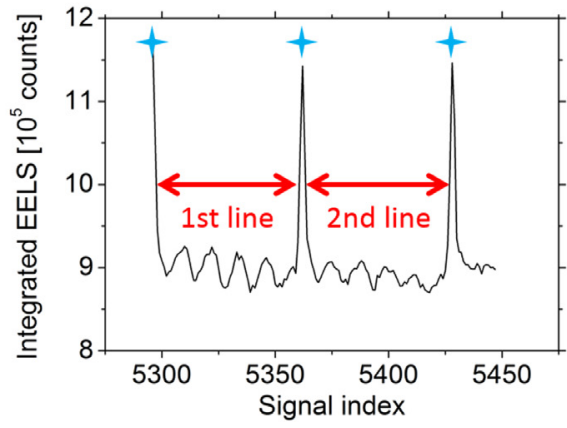

Fig. 2. Overview ADF-STEM image of the [100] oriented STO lamella including the 64 by 128 pixels ROI and the vacuum marker position which are scanned with $1000 \mathrm{px} / \mathrm{s}$ acquisition rate (a) as well as excerpts of the simultaneously collected ADF detector output voltage (b) and integrated EELS spectra (c).

limits the scattering angle of the collected electrons hence the integrated EELS spectra mimic a bright field signal. The blue stars in both line profiles clearly indicate the vacuum marker position such that the signals in between can be attributed to the corresponding lines of the ROI. More technically, each point is assigned to be in vacuum if the signal is lower than $1.5 \mathrm{~V}$ or greater than $10.5 \times 10^{5}$ counts for ADF and EELS, respectively.

Following the reconstruction procedure mentioned before leads to Fig. 3 (a) and (b). For the sake of comparison, the integrated EELS signal has been inverted in order to mimic a dark field signal. As marked by the colored arrows, flag-like artifacts can either coincide or appear in only one the signals. Whereas the latter is a strong hint for acquisition rate instabilities of the respective detectors, the former could be both explained by irregularities of the beam movement clocking as well as general instabilities of the microscope leading to commonly observed distortions in STEM images [8]. Notwithstanding 


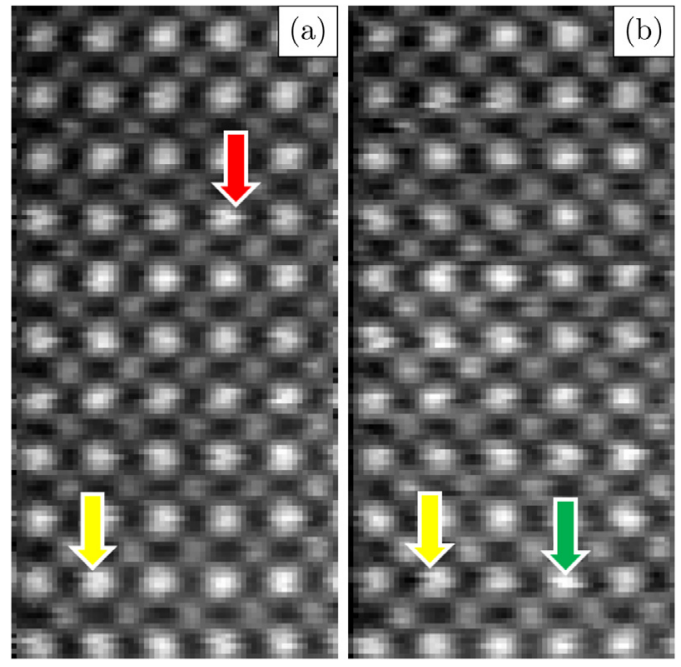

Fig. 3. A posteriori reconstructed ADF (a) and inverted integrated EELS (b) signal corresponding to the reference in Fig. 2. Exemplary artifacts emerging only in the ADF signal (red), only in the EELS signal (green) and which coincide (yellow) are marked with arrows. (For interpretation of the references to colour in this figure legend, the reader is referred to the web version of this article.)

these occasional flaws, it can be concluded that a posteriori synchronized images are of comparable quality of hardware-synchronized data sets as will be demonstrated below.

In order to compare the method to state of the art commercial products, the hardware-synchronized Gatan Spectrum Imaging (SI) tool has been used to obtain the data shown in Fig. 4. Clearly, it is less likely to find flags in the hardware-synchronized images and those that appear seem to coincide in both signals as marked by the yellow arrows hence one cannot directly relate the artifacts to clocking instabilities.

\subsection{Error quantification}

Since the eye-guided search for artifacts in the images is rather qualitative and highly subjective, a more thorough error estimation will be given in this subsection. First, we note that resolution and accuracy in the vertical direction is determined by the microscope and does not

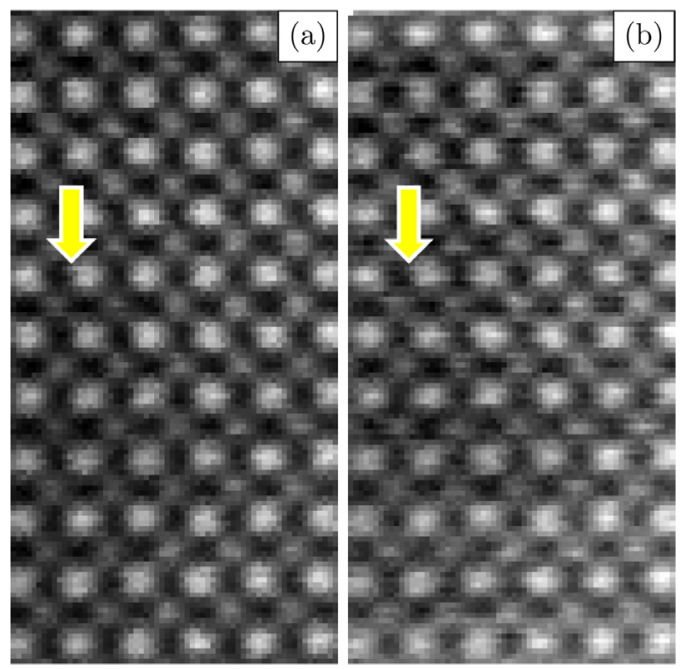

Fig. 4. ADF (a) and inverted integrated EELS (b) signal close to the reference in Fig. 2 obtained with the hardware-synchronized Gatan SI tool. A coinciding artifact is marked with yellow arrows. (For interpretation of the references to colour in this figure legend, the reader is referred to the web version of this article.) (a)

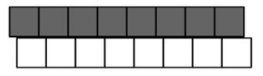

(c)

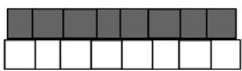

(b)

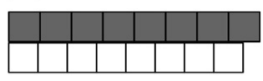

Fig. 5. Visualization of the synchronization error sources: The grey and white boxes represent beam dwelling and signal integration periods. (a) shows a constant offset due to the missing phase lock, (b) an increasing delay due to different mean clocking periods, and (c) a stochastic scattering of the respective periods due to irregularities.

suffer from a posteriori synchronization. The horizontal direction, however, is affected due to the accidental inclusion of signals from different scanning points during one acquisition. Three independent sources of experimental uncertainty can be identified, i.e. (i) the missing phase lock (experimental uncertainty $\sigma_{\text {ph }}$ ), (ii) a possible difference in the mean clocking periods of different recording devices $\left(\sigma_{\text {per }}\right)$, and (iii) irregularities in their clocking processes $\left(\sigma_{\text {irr }}\right)$. The total experimental uncertainty $\sigma$ can then be written as

$\sigma=\sqrt{\sigma_{\text {ph. }}^{2}+\sigma_{\text {per. }}^{2}+\sigma_{\text {irr. }}^{2}}$.

A graphical illustration of the error sources is given in Fig. 5, where grey boxes refer to the dwelling periods of the beam and white boxes signal integration periods. Firstly, the missing phase lock leads to a uniformly distributed spread between $50 \%$ with the previous and $50 \%$ with the next pixel hence the standard deviation of the resulting error is given by that of the uniform distribution within the interval $[-0.5,0.5]$ times the pixel spacing $\Delta x$, i.e. $\sigma_{\text {ph. }}=\Delta x / \sqrt{12}$. Secondly, the maximum spread with neighboring points within a line due to different mean clocking periods is given by the number of scanning points $N$ per line times the relative difference of the periods. Estimating the latter with $\left|\bar{N}_{\text {acq. }}-N\right| / N$, where $N_{\text {acq. }}$ is the averaged number of acquisitions per line, leads to $\sigma_{\text {per. }}=\Delta x \cdot\left|\bar{N}_{\text {acq. }}-N\right|$. Finally, the irregularities in the clocking processes can be estimated by the standard deviation of the acquisitions per line times the pixel spacing $\sigma_{\text {irr. }}=\Delta x \cdot \sigma_{N_{\text {acq }}} / \sqrt{2}$. Note that the division by $\sqrt{2}$ accounts for the fact that both the irregularities of the first and the last pixel contribute equally to the fluctuation in $N_{\text {acq. }}$. It is worth mentioning that $\sigma_{\mathrm{ph}}$. represents the theoretical limit of the proposed method for perfectly regular and coherent clocking processes and therefore the margin to a perfect time synchronization. For the sake of quantification, five acquisitions have been performed analogously to the one shown in Fig. 3 yielding the averaged error contributions in Table 2 .

A second and rather experimental approach to assess the error resulting from a posteriori synchronization is to compare the data to hardware-synchronized acquisitions quantitatively. Due to the similarity of the inverted integrated EELS and ADF signals, a high CrossCorrelation Coefficient (CCC) of the reconstructed images is expected. Note that the first and last pixels of each line have been omitted in the following calculations since they contain strongly deviating signal from the marker position. In fact, this reduces the fraction of useful data in the a posteriori synchronized images to $96.9 \%$ which will be taken into account later in the conclusion. To maintain equal conditions, the same omission has been performed to hardware-synchronized data as well. The mean CCC of the reduced a posteriori synchronized images obtained by five acquisitions is $0.867(12)$. Using Gatan SI, a slightly higher value of $0.886(8)$ could be achieved with an average acquisition time of $9.6 \mathrm{~s}$. The reduction of CCC caused by asynchronicities can be modeled by shifting the horizontal sampling points in one of the hardware-synchronized EELS images according to a random variable. Due to its similarity to a missing phase lock, a uniform distribution around the initial sampling point has been used. The signals of non-integer shifted sampling positions have been interpolated with next neighbors. For 


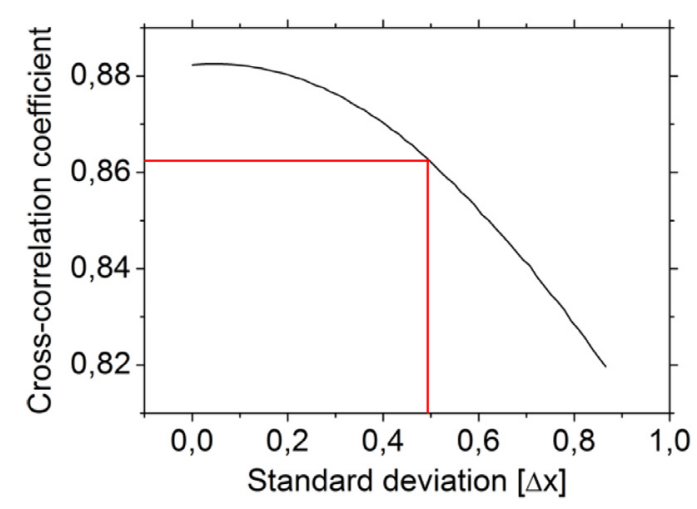

Fig. 6. Cross-correlation coefficient (averaged over 100 runs) of hardwaresynchronized ADF and inverted integrated EELS images with respect to the standard deviation of a uniformly distributed shift in the EELS sampling position. The signals of non-integer shifted sampling positions have been interpolated with next neighbors. The red lines mark the point at which the initial value is reduced by 0.02 . (For interpretation of the references to colour in this figure legend, the reader is referred to the web version of this article.)

each fixed standard deviation of the distribution the CCC between the shifted EELS and unaltered ADF images has been averaged over 100 runs to reduce probabilistic scattering and can be found in Fig. 6. In remarkable consistence with the values of Table 2 , the margin of 0.02 in the CCC can be resembled with a standard deviation of roughly $0.49 \Delta x$ as marked by the red lines.

\subsection{The Method's versatility}

In order to demonstrate how easily a third signal acquisition can be included, the acquisition rate was reduced to $10 \mathrm{px} / \mathrm{s}$ to achieve an acceptable EDX signal-to-noise ratio. Scanning the ROI shown in Fig. 7 with 32 by 32 pixels results in a total acquisition time of $102 \mathrm{~s}$. The localization of the marker signal in the ADF and EELS signal was done analogously to the first example whereas for EDX the Cu K-edge counts related to the support grid on which the lamella was mounted were used. Once the correlation between signal index and spatial position has been obtained, any analysis, e.g. chemical mapping, can be performed. Fig. 8 shows the reconstructed ADF signal (a) as well as a map of the integrated EELS Ti L-edge obtained after power law background subtraction (b), the integrated EDX Sr K- and L-edge (d) and the integrated EDX Ti-K edge (e). Even though the statistics of the EDX map is still poor compared to ADF and EELS and the effect of small drift is apparent, the atomic structure of STO is clearly visible in all images. As the superimposed color images in figure 8 (c) and (f) with Ti contributions in red illustrate, consistent chemical contrast is achieved combining the ADF signal with EELS respectively the two EDX edges. Given the lower scanning rate, the predominant distortion of the images is caused by sample drift.

\section{Summary and conclusion}

We have presented a scanning pattern allowing for a posteriori

Table 1

Chosen energy windows for EELS background fitting, EELS Ti L-edge integration after background subtraction as well as all EDX edges.

\begin{tabular}{lll}
\hline & $E_{\min }[\mathrm{eV}]$ & $E_{\min }[\mathrm{eV}]$ \\
\hline EELS background & 384 & 448 \\
EELS Ti L-edge & 451 & 515 \\
EDX Sr L-edge & 1,709 & 1,989 \\
EDX Ti K-edge & 4,362 & 5,069 \\
EDX Sr K-edge & 13,856 & 16,237 \\
\hline
\end{tabular}

Table 2

Error contributions of the missing phase lock $\sigma_{\text {ph. }}$, different mean clocking periods $\sigma_{\text {per. }}$, and clocking irregularities $\sigma_{\text {irr. }}$ to the total error $\sigma$ of the $a$ posteriori synchronization of ADF and EELS signals.

\begin{tabular}{lll}
\hline & ADF & EELS \\
\hline$\sigma_{\text {ph. }}[\Delta x]$ & 0.29 & 0.29 \\
$\sigma_{\text {per. }}[\Delta x]$ & 0.27 & 0.28 \\
$\sigma_{\text {irr. }}[\Delta x]$ & 0.3 & 0.32 \\
$\sigma[\Delta x]$ & 0.49 & 0.52 \\
\hline
\end{tabular}

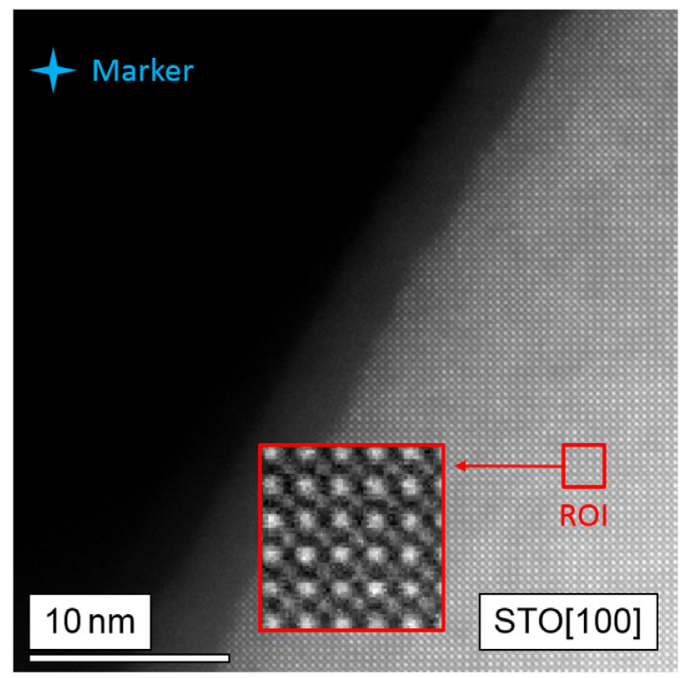

Fig. 7. Overview ADF-STEM image of the [100] oriented STO lamella including the 32 by 32 pixels ROI and the vacuum marker position which are scanned with $10 \mathrm{px} / \mathrm{s}$ acquisition rate.
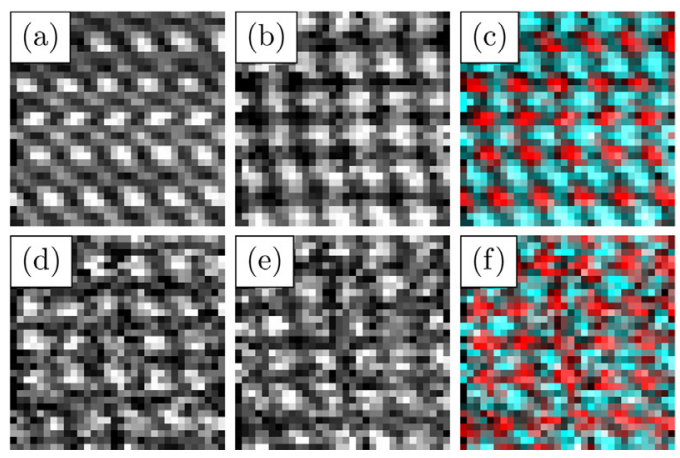

Fig. 8. A posteriori reconstructed signals corresponding to the reference in Fig. 7: ADF signal (a), integrated EELS Ti-L edge after background subtraction (b), sum of EDX Sr K- and L-edge (d) and EDX Ti K-edge (e) counts. Details about the respective energy windows are given in Table 1 . (c) shows a superimposed color image with (b) in red and (a) in cyan. (f) shows a superimposed color image with (e) in red and (d) in cyan. (For interpretation of the references to colour in this figure legend, the reader is referred to the web version of this article.)

synchronization of STEM data by marking the end of each line with a distinct signal of a marker position. Images of simultaneously collected ADF detector voltages and EELS spectra have been reconstructed yielding atomic resolution for acquisition rates up to $1000 \mathrm{px} / \mathrm{s}$ evidencing the applicability to modern fast digital cameras.

\subsection{Comparison with hardware synchronization}

Compared to hardware-synchronized setups, the method causes an additional spread in scanning direction which is in theory at least $1 / \sqrt{12}$ 
times and in practice roughly 0.5 times the pixel spacing. Notwithstanding this limitation, a posteriori synchronization adds value to existing solutions in terms of speed: As mentioned in the previous section, the acquisition of a 64 by 128 pixels frame with an acquisition rate of $1000 \mathrm{px} / \mathrm{s}$ took $8.3 \mathrm{~s}$. In order to achieve such a high EELS readout rate, the exposure time was set to $440 \mu$ s. Using the same exposure time and experimental conditions with the Gatan SI tool results in a total acquisition time of $9.6 \mathrm{~s}$. After accounting for the omission of the first and last pixels in the a posteriori images this corresponds to a speed gain of $12 \%$ and hence the detection of more signal per incoming electron. Particularly in the field of beam sensitive materials, the resulting reduction of electron dose can be very advantageous. In addition, the ordering of the scanning lines could be changed with an ease or even probed in a sparse sampling approach like it has been presented before [9].

\subsection{The Method's versatility}

Furthermore, the high versatility of the method has been proven by collecting and synchronizing EDX spectra in addition, i.e. we collected signals which could not be combined in our setup before. In fact, in the second example, data of three sources was collected using two independently running computers and the direct read-out of the multimeter. Consequently, detectors of different manufacturers running on different platforms can be combined without any constraints.

One possible application that we would like to emphasize at this point is the collection of entire diffraction patterns for each scanning position also referred to as 4DSTEM. Since the diffraction patterns contain a large variety of information this approach can be used to map physical quantities such as electric or magnetic fields as well as strain [10-13]. The popularity of 4DSTEM has raised tremendously due to the improvement of digital cameras, i.e. direct detectors are capable of recording several thousand diffraction patterns per second - an acquisition rate to which the presented method has proven to be applicable.

\subsection{Conditions and limitations}

The only necessary conditions are the possibility to control the electron beam as well as the availability of a marker position exhibiting a distinct signal. The latter can consist of only one point as in the examples shown here, but allows also for some creative workarounds such as using a more complicated template similar to a Morse code or choosing a marker that occurs only in the center of lines and exploiting the knowledge of the approximate amount of signals per line to locate its beginning and end. Another possible solution to the non-existence of a distinct area is to use the electron beam to drill a hole in the sample which we have successfully tested. Still, in the case of a low signal to noise ratio, the exact localization of the marker sequence might become difficult leading to additional distortions in the reconstructed images.

The effect of sample drift becomes apparent using small acquisition rates as shown in Fig. 8. The robustness of the method against this depends on the localization of the marker sequence, i.e. if the signals of the latter change significantly due to small sample movements the reconstruction can be difficult. On the other hand, assuming that the signal evolution of the marker sequence under translation is known, one could even use this effect to correct for sample drift.

In conclusion, we have described and implemented a simple but powerful a posteriori synchronization scheme which can be considered as a trade-off between higher speed and flexibility on the upside and lower spatial resolution in scanning direction on the downside: The method allows for simultaneous device-independent recording of arbitrarily many signals during STEM acquisitions with up to $1000 \mathrm{px} / \mathrm{s}$ and offers, in the regime of small integration times, a speed gain of $12 \%$. The spatial blurring in scanning direction was quantified to be only half a pixel spacing.

\section{Author Contributions}

The original idea was conceived by S.L and V.R.: The design of experiments was the result of fruitful discussions including all authors; T.M. conducted the measurements and wrote the DM script with advise of V.R.; M.S. and V.R. supervised the study. The manuscript was written by T.M. under revision of S.L., M.S. and V.R.; All authors read and agreed on the written paper.

\section{Conflicts of Interest}

Conflicts of Interest: The authors declare no conflict of interest.

\section{Acknowledgments}

We thank Christian Jooss and the anonymous reviewers sincerely for carefully reading the manuscript and helping to improve its quality. Funding by the Deutsche Forschungsgemeinschaft (DFG) via the CRC 1073, project B02 and Z02, is gratefully acknowledged.

\section{References}

[1] A. Crewe, The physics of the high-resolution scanning microscope, Rep. Prog. Phys. 43 (5) (1980) 621.

[2] M. Isaacson, D. Johnson, The microanalysis of light elements using transmitted energy loss electrons, Ultramicroscopy 1 (1) (1975) 33-52.

[3] C. Lyman, D. Williams, J. Goldstein, X-Ray detectors and spectrometers, Ultramicroscopy 28 (1-4) (1989) 137-149.

[4] E.J. Kirkland, Fine tuning an aberration corrected adf-stem, Ultramicroscopy 186 (2018) 62-65.

[5] A.-C. Milazzo, G. Moldovan, J. Lanman, L. Jin, J.C. Bouwer, S. Klienfelder, S.T. Peltier, M.H. Ellisman, A.I. Kirkland, N.-H. Xuong, Characterization of a direct detection device imaging camera for transmission electron microscopy, Ultramicroscopy 110 (7) (2010) 741-744.

[6] M. Krajnak, D. McGrouther, D. Maneuski, V. O'Shea, S. McVitie, Pixelated detectors and improved efficiency for magnetic imaging in stem differential phase contrast, Ultramicroscopy 165 (2016) 42-50.

[7] T. Meyer, S. Lopatin, M. Seibt, V. Roddatis, Supplementary Material, Ultramicroscopy.

[8] S. Ning, T. Fujita, A. Nie, Z. Wang, X. Xu, J. Chen, M. Chen, S. Yao, T.-Y. Zhang, Scanning distortion correction in stem images, Ultramicroscopy 184 (2018) 274-283, https://doi.org/10.1016/j.ultramic.2017.09.003.

[9] L. Kovarik, A. Stevens, A. Liyu, N.D. Browning, Implementing an accurate and rapid sparse sampling approach for low-dose atomic resolution stem imaging, Appl. Phys. Lett. 109 (16) (2016) 164102.

[10] K. Müller, F.F. Krause, A. Béché, M. Schowalter, V. Galioit, S. Löffler, J. Verbeeck, J. Zweck, P. Schattschneider, A. Rosenauer, Atomic electric fields revealed by a quantum mechanical approach to electron picodiffraction, Nat. Commun. 5 (2014) 5653.

[11] S. Lopatin, Y.P. Ivanov, J. Kosel, A. Chuvilin, Multiscale differential phase contrast analysis with a unitary detector, Ultramicroscopy 162 (2016) 74-81.

[12] D. Cooper, T. Denneulin, N. Bernier, A. Béché, J.-L. Rouvière, Strain mapping of semiconductor specimens with nm-scale resolution in a transmission electron microscope, Micron 80 (2016) 145-165.

[13] C. Gammer, C. Ophus, T.C. Pekin, J. Eckert, A.M. Minor, Local nanoscale strain mapping of a metallic glass during in situ testing, Appl. Phys. Lett. 112 (17) (2018) 171905. 


\section{Chapter 5}

\section{Inverse Modelling of Octahedral Tilts and Distortions in Bulk PCMO}

In this chapter, the structural model described in chapter 2, i.e. Eq. (2.3), (2.4), and (2.6), shall be applied to existing refined neutron diffraction data in the literature in order to study if relevant structural modes can be extracted by inverse modelling. More specifically, the results from [51] (for $x=0$ and elevated temperatures) and [20,52] (for $0 \leq x \leq 0.5$ at room temperature), which have already been presented in Fig. 2.6 will be used as a control set to develop optimisation strategies for extraction of the octahedral tilt angle $\theta$ as well as the antiferrodistortive $q_{3}$ mode. Subsequently, this strategy will be applied to non-refined data published in [161] suggesting that $q_{3}$ is enhanced in bulk PCMO with $x=0.1$ at low temperatures due to orbital ordering.

Given the expressions for $a, b$, and $c$ as functions of $\left(n_{2}, \theta, d_{0}, q_{2}, q_{3}\right)$ - by using the basis chosen in Eq. (2.11)-(2.13), the following inverse problem is well-defined:

$$
\begin{array}{r}
\min _{n_{2}, \theta, d_{0}, q_{2}, q_{3}}\left(\left(a\left(n_{2}, \theta, d_{0}, q_{2}, q_{3}\right)-\right.\right. \\
\left.a_{0}\right)^{2}+\left(b\left(n_{2}, \theta, d_{0}, q_{2}, q_{3}\right)-b_{0}\right)^{2} \\
\left.+\frac{1}{2}\left(c\left(n_{2}, \theta, d_{0}, q_{2}, q_{3}\right)-c_{0}\right)^{2}\right) .
\end{array}
$$

Here, $a_{0}, b_{0}$, and $c_{0}$ represent given values of the $\mathrm{Pbnm}$ lattice constants, e.g. experimentally obtained, and solutions of Eq. (5.1) define optimal parameters resembling the latter best in the underlying model of untwisted 
$\mathrm{MnO}_{6}$ octahedra. Importantly, a unique definition of five variables by only three observables is unlikely and the presented inverse problem is expected to be prone to overfitting in the presence of experimental noise. Thus, the
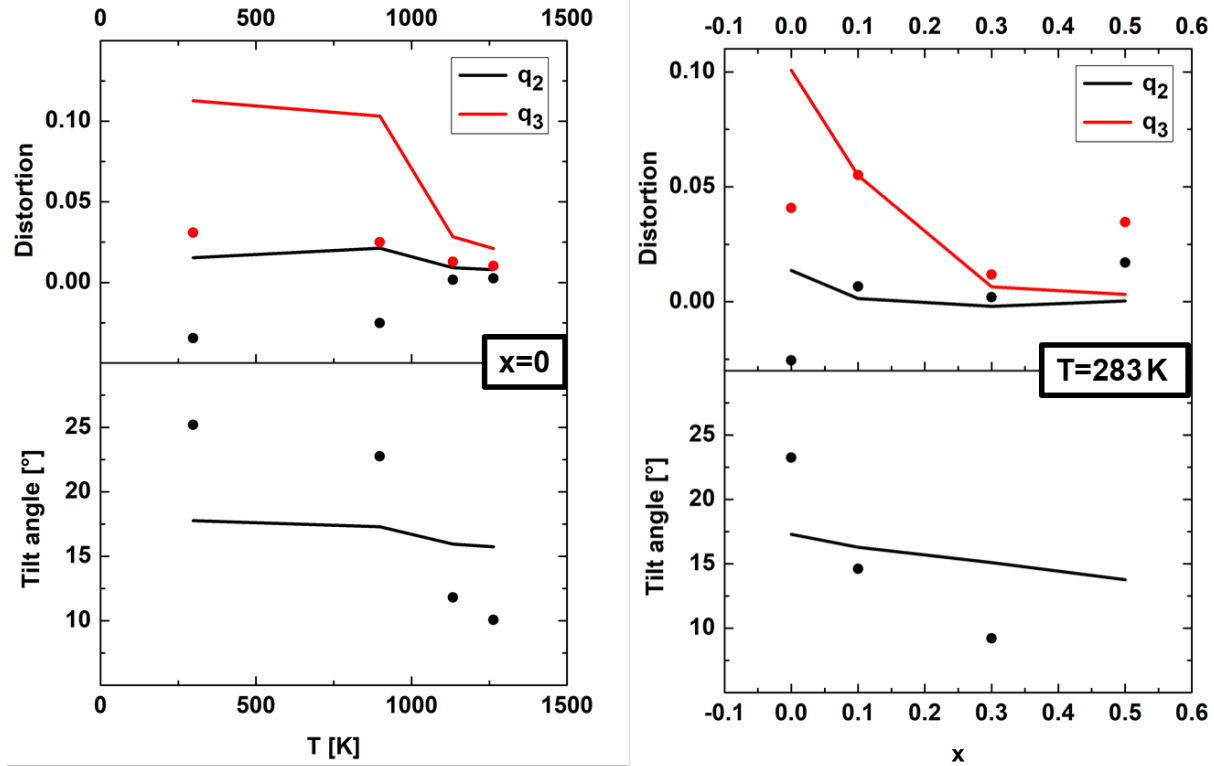

Figure 5.1: Optimal solutions of Eq. (5.1) for $n_{2}=-0.78$ and free parameters $\left(\theta, d_{0}, q_{2}, q_{3}\right)$ applied to the data in [51] (left, $\left.x=0\right)$ resp. [20, 52] (right, $T=283 \mathrm{~K}$ ). Circles resp. lines represent the fit results resp. the data obtained by the refinement.

reduction of variables to fixed parameters is desirable. As demonstrated previously in Fig. 2.6, $n_{2}$ shows generally little variations and will consequently be fixed to $n_{2}=-0.78$, i.e. its mean value in the mentioned figure, in the following considerations. Fig. 5.1-5.3 show the results of three approaches (points) applied to the data of [51] and [20,52] $(0 \leq x \leq 0.5)$ and compared with the refined values (lines): Firstly, a simultaneous fit of $\left(\theta, d_{0}, q_{2}, q_{3}\right)$ is presented in Fig. 5.1 showing the expected overfitting behaviour, i.e. physically unreasonable structural parameters yielding residual values in Eq. (5.1) of numerically zero. Secondly, a fit of $\left(\theta, q_{2}, q_{3}\right)$ with $d_{0}$ being fixed to the values obtained by the refinement is presented in Fig. 5.2 resulting in a good agreement between fit and experiment for $\theta$ while $q_{2}$ and $q_{3}$ still show strong deviations. Thirdly, a subsequent fit of $q_{3}$ with $d_{0}$ and $q_{2}$ being fixed to the refined values as well as $\theta$ to the results obtained in 

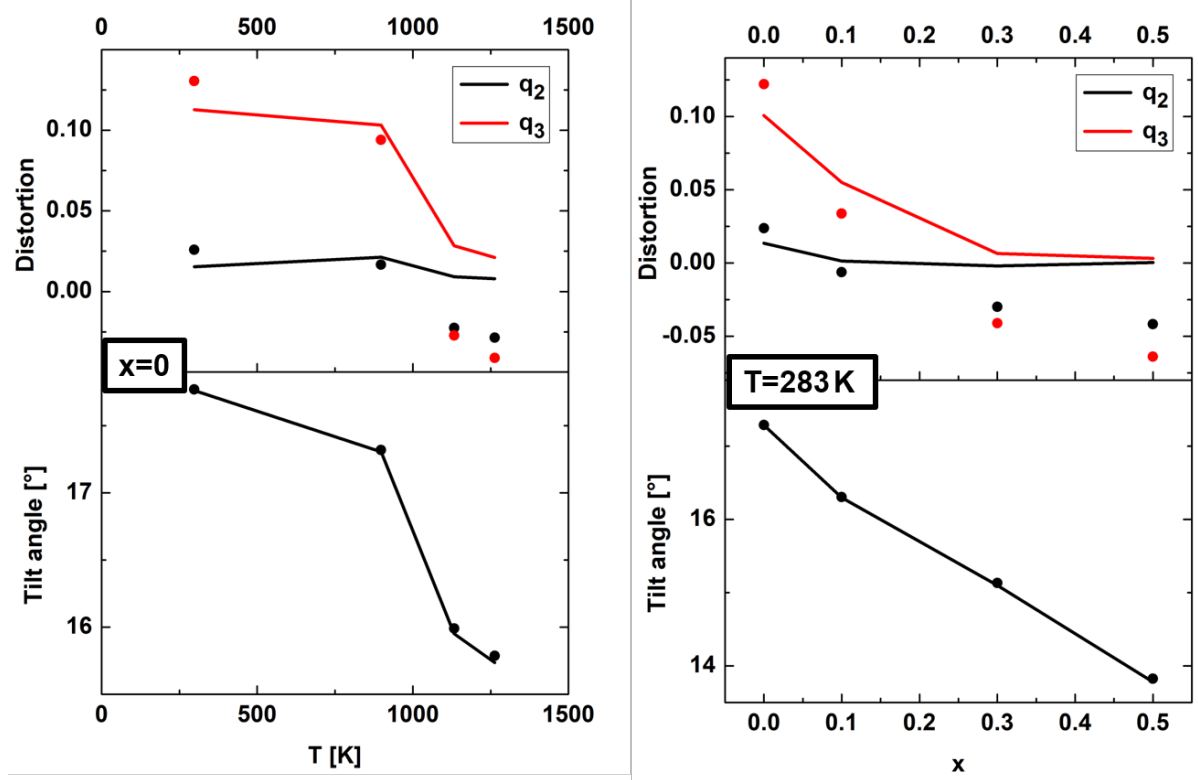

Figure 5.2: Optimal solutions of Eq. (5.1) for $n_{2}=-0.78$, fixed experimental values for $d_{0}$ and free parameters $\left(\theta, q_{2}, q_{3}\right)$ applied to the data in [51] (left, $x=0$ ) resp. [20, 52] (right, $T=283 \mathrm{~K}$ ). Circles resp. lines represent the fit results resp. the data obtained by the refinement.

the second approach is presented in Fig. 5.3. Please note that due to the reduction of fitting variables, a reduced chi squared analysis including an error estimation is feasible in the latter approach, i.e. minimisation of

$$
\begin{array}{r}
\min _{q_{3}}\left(\left(a\left(n_{2}, \theta, d_{0}, q_{2}, q_{3}\right)-a_{0}\right)^{2}+\left(b\left(n_{2}, \theta, d_{0}, q_{2}, q_{3}\right)-b_{0}\right)^{2}\right. \\
\left.+\frac{1}{2}\left(c\left(n_{2}, \theta, d_{0}, q_{2}, q_{3}\right)-c_{0}\right)^{2}\right) /\left(2 \sigma_{a b c}^{2}\right) .
\end{array}
$$

Here, $\sigma_{a b c}=1.5 \mathrm{pm}$ was used in accordance with the RMS values obtained in Fig. 2.6. Altogether, the findings suggest that the chosen basis of distortions separates $\left(\theta, d_{0}, q_{2}, q_{3}\right)$ in two rather independent pairs of strongly coupled variables, i.e. $\left(\theta, d_{0}\right)$ and $\left(q_{2}, q_{3}\right)$, reflecting that both a decrease resp. increase of $d_{0}$ resp. $\theta$ reduce all lattice constants simultaneously while $q_{2}$ and $q_{3}$ cause expansions and contractions. In fact, while the naive approach of fitting all four quantities simultaneously fails awfully (Fig. 5.1), fixing $d_{0}$ leads to a precise extraction of $\theta$ (Fig. 5.2) and a remaining overfitting of 


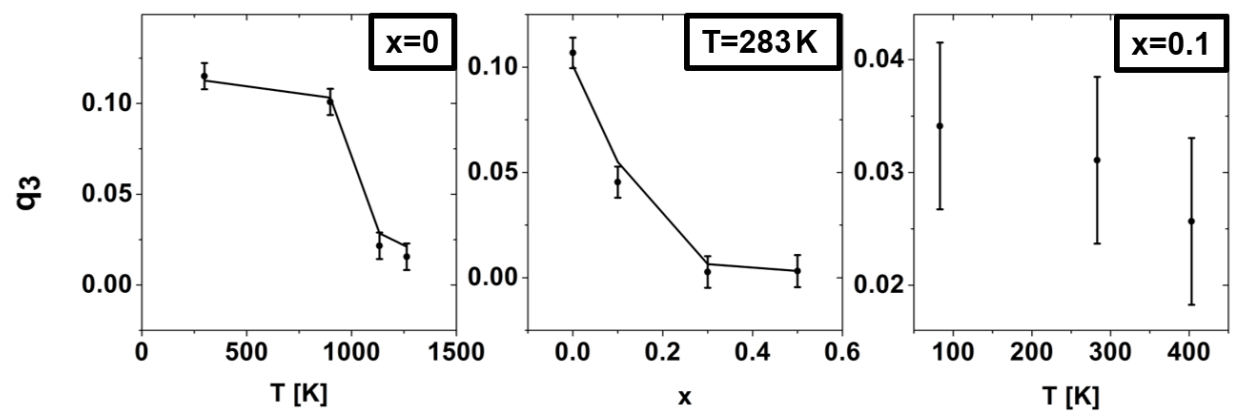

Figure 5.3: Optimal solutions of Eq. (5.1) with $q_{3}$ as the only free parameter and $n_{2}=-0.78 . d_{0}$ and $q_{2}$ were set to the experimentally determined values given in [51] (left, $x=0$ ) resp. [20, 52] (center, $T=283 \mathrm{~K}$ ) and $\theta$ was fixed to the values obtained with free parameters $\left(\theta, q_{2}, q_{3}\right)$ and presented in Fig. 5.2. As no refined data exist for the data from [161] (right, $x=0.1$ ), $d_{0}$ was fixed to the value from [20] and $q_{2}$ was set to zero. Circles resp. lines represent the fit results resp. data obtained by the refinement if existing.

$\left(q_{2}, q_{3}\right)$. Fixing $q_{2}$ in addition and keeping the results of $\theta$ yields precise values for $q_{3}$ as well. Lastly, as shown on the right in Fig. 5.3, this strategy was transferred to non-refined data at low temperatures and $x=0.1$ given in [161]. Please note that a different space group is used in the latter reference leading to a cyclic rotation of the lattice parameters. As neither $d_{0}$ nor $q_{2}$ are known, they are assumed to be constant, i.e. $d_{0}$ is fixed to the room temperature value of $x=0.1$ in [20] and $q_{2}$ to zero. Since the resulting values of $\theta$ show only non-significant variations of roughly $0.1^{\circ}$ from the room temperature value $16.6^{\circ}$, solely $q_{3}$ is presented. Here, a slight increase on the order of the uncertainties is observed towards low temperatures, which might indicate an enhanced JT effect due to orbital ordering as discussed in chapter 2. Importantly, due to the assumptions made, this interpretation based on the solitary data presented in this chapter should be taken with care. However, it is very consistent with and thus supporting the findings in [28]. Moreover, the findings show that rather few observables are necessary to extract the octahedral configuration in PCMO, which could pave the road for robust electron diffraction refinements in future studies. 


\section{Chapter 6}

\section{Exploring the Phase Diagram of PCMO Thin Films}

In this chapter, parts of the phase diagram of PCMO thin films shall be explored by means of electron diffraction techniques and compared to the results obtained on bulk samples discussed in chapter 2. All investigated macroscopic samples have been prepared by B. Kressdorf using ion beam sputtering and relevant parameters are given in Tab. A.1. More specifically, two Ca concentrations of $x=0.34$ and $x=0.1$ are studied representing candidates for the emergence of charge (and orbital) ordering in the medium and orbital ordering in the low doping regime, respectively.

\subsection{Charge Ordering for $x=0.34$}

In order to demonstrate charge ordering (CO) in a nominally $100 \mathrm{~nm}$ thick PCMO film $(x=0.34)$ on STO, a planview orientated TEM sample is advantageous since mainly $c$-out-of-plane growth direction [17] and the predominant emergence of a $2 b$ superstructure [57] is expected. Consequently, a $c$-oriented lamella guarantees the inclusion of large scattering volumes with $b$ orthogonal to the electron beam. Therefore, the following two-step sample preparation was used for the herein presented experiment:

- A $2 \times 1.5 \mathrm{~mm}^{2}$ block was cut from the center of the macroscopic sample using a diamond saw, glued upside down with mounting wax on a stainless steel stub, and ground to a thickness of $20 \mu \mathrm{m}$ with P2400 
paper on a disc grinder. Subsequently, the specimen was cleaned first in acetone and then in isopropanol for several minutes, glued upside down with Gatan G1 glue on a copper support grid, and milled from the backside with the Gatan precision ion polishing system (PIPS) until a hole emerged using an acceleration voltage of $4 \mathrm{kV}$.

- The electron transparent sample mounted on the copper grid was inserted into the FIB and a small piece was cut out and transferred to a silicon nitride membrane which had been evaporated with a few hundred nanometer gold before. The lamella was mounted across a hole that has been milled with the ion beam before. During this process, the ion beam exposure has been reduced as much as possible to prevent damage of the region of interest.

Please note that the second step is generally not necessary to investigate $\mathrm{CO}$ and was primarily done as preparation for a planned follow-up pumpprobe experiment in an ultrafast TEM, in which the gold layer is supposed to act both as a heat sink as well as a mirror for the incoming pumping laser. Nevertheless, once the lamella has been transferred, this geometry is even for the sake of a pure cooling experiment beneficial as it eliminates the risk of crack formation and breaking of the mechanically very unstable conventionally prepared sample during further transfers.

Using the Gatan cooling stage mentioned in chapter 3 to approach low temperatures yields the expected $2 b$ superstructure at $100 \mathrm{~K}$ as shown in the upper left selective area electron diffraction (SAED) pattern of Fig. 6.1. The real space region corresponding to the diffraction pattern can be found in Fig. A.10 and the thickness in the investigated area was determined with Eq. (3.1) to approximately $180 \mathrm{~nm}$ using $\lambda=107.5 \mathrm{~nm}$ as an average for PCMO and STNO [162]. Furthermore, Fig. 6.1 visualises the masking approach which shall be used in this section to quantify the intensity of the $\mathrm{CO}$ peaks: For a given radius $r_{\mathrm{d}}$, a circular mask is created around all orders of the $\mathrm{CO}$ reflections and the averaged disc intensity $I_{\mathrm{d}}$ is defined as:

$$
I_{\mathrm{d}}\left(r_{\mathrm{d}}, T\right)=\int_{\Sigma\left(r_{\mathrm{d}}\right)} I\left(k_{x}, k_{y}, T\right) \mathrm{d} k_{x} \mathrm{~d} k_{y} / \int_{\Sigma\left(r_{\mathrm{d}}\right)} \mathrm{d} k_{x} \mathrm{~d} k_{y},
$$

where $I\left(k_{x}, k_{y}, T\right)$ is the recorded diffraction intensity at the position $\left(k_{x}, k_{y}\right)$ in reciprocal space and temperature $T$ and $\Sigma\left(r_{\mathrm{d}}\right)$ the circular domain around the superstructure reflections is exemplary visualised in Fig 6.2 and defined by

$$
\Sigma\left(r_{\mathrm{d}}\right)=\left\{\left(k_{x}, k_{y}\right):\left|\left(k_{x}, k_{y}\right)-\left(i \cdot a^{*},(j+0.5) \cdot b^{*}\right)\right|^{2} \leq r_{\mathrm{d}}^{2} ; i, j \in \mathbb{Z}\right\} .
$$




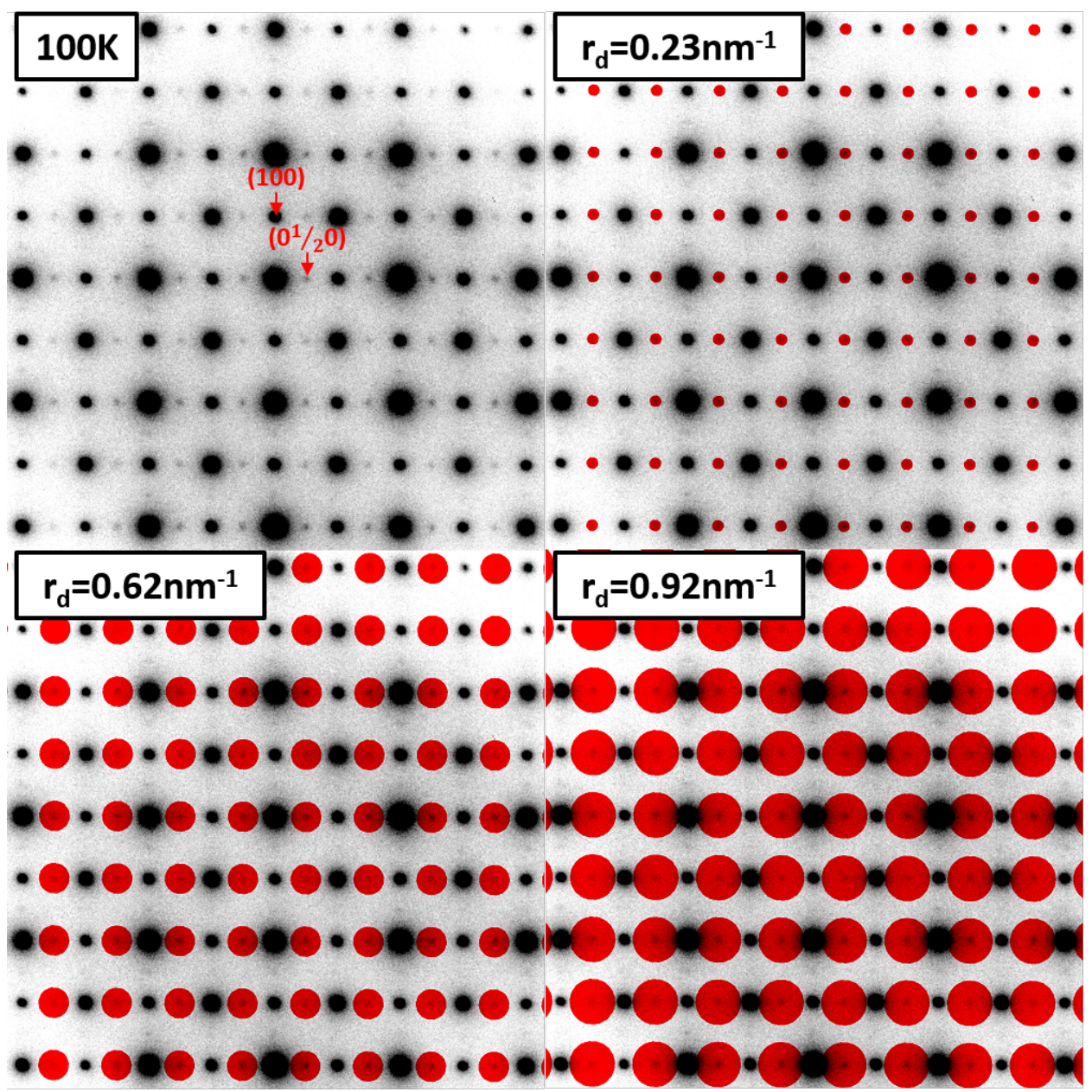

Figure 6.1: SAED pattern of charge ordered PCMO $(x=0.34)$ with the expected $2 b$ superstructure at $100 \mathrm{~K}$ (top left). In addition, the mask definition given in Eq. (6.2) is visualised for three different values of $r_{\mathrm{d}}$. 


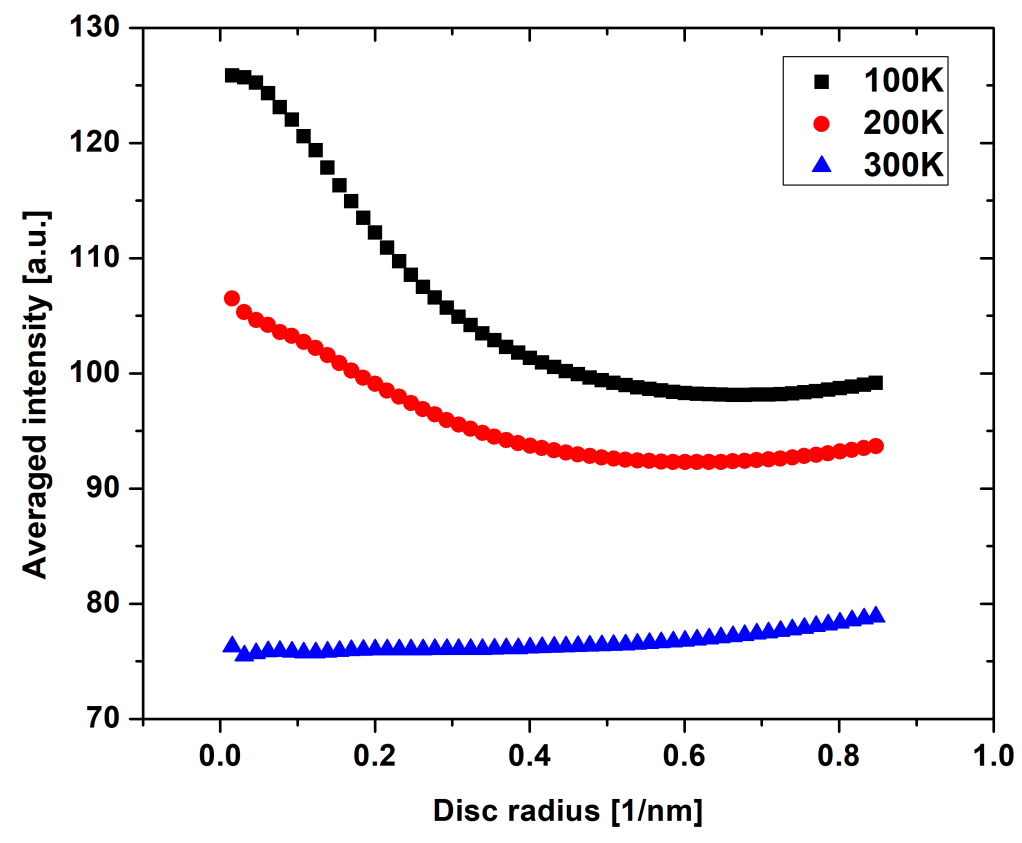

Figure 6.2: Averaged intensity $I_{\mathrm{d}}$ as defined in Eq. (6.1) with respect to the disc radius $r_{\mathrm{d}}$ for selected temperatures.

Here, $a^{*}$ resp. $b^{*}$ are the reciprocal lattice parameters of PCMO. The resulting averaged intensity for three exemplary temperatures is given in Fig. 6.2 revealing that for low temperatures, in the presence of charge ordering, $I_{\mathrm{d}}$ increases towards smaller radii. In addition, as can be seen most clearly in the case of $T=300 \mathrm{~K}, I_{\mathrm{d}}$ contains even for small radii still a significant contribution of the decaying intensity of the main diffraction peaks. In order to correct for this effect, $I_{\mathrm{d}}(T=300 \mathrm{~K})$ scaled to the value at $r_{\mathrm{d}}=0.85 \mathrm{~nm}^{-1}$ is subtracted resulting in the averaged excess intensity due to the CO peaks:

$$
I_{\mathrm{CO}}\left(r_{\mathrm{d}}, T\right)=I_{\mathrm{d}}\left(r_{\mathrm{d}}, T\right)-I_{\mathrm{d}}\left(r_{\mathrm{d}}, 300 \mathrm{~K}\right) \cdot \frac{I_{\mathrm{d}}\left(0.85 \mathrm{~nm}^{-1}, T\right)}{I_{\mathrm{d}}\left(0.85 \mathrm{~nm}^{-1}, 300 \mathrm{~K}\right)} .
$$

The corresponding curves for the entire temperature series taken in the experiment are shown in Fig. 6.3. As expected, $I_{\mathrm{CO}}$ increases generally with decreasing temperature whereas the shape of the curves remains unchanged, which can be confirmed by plotting normalised profiles. For small radii, the 


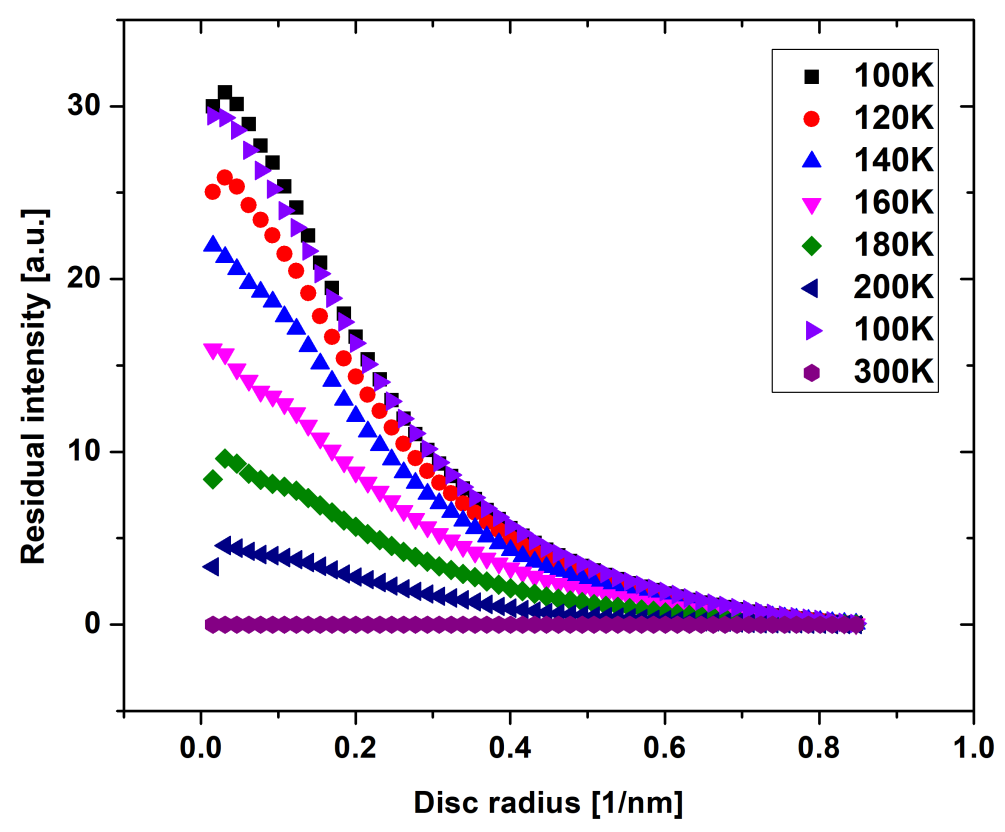

Figure 6.3: Excess intensity due to charge ordering as defined in Eq. (6.3) with respect to the disc radius $r_{\mathrm{d}}$ for all temperatures used in the cooling experiment.

curves become increasingly noisy since only a few pixels lie within the masked area. Thus, $I_{\mathrm{CO}}$ is evaluated at $r_{\mathrm{d}}=0.3 \mathrm{~nm}^{-1}$ as a measure for the CO peak intensity and illustrated in Fig. 6.4 after being normalised to the value at $T=100 \mathrm{~K}$. In addition, the normalised averaged intensity of an analogously constructed mask around the main peaks of the diffraction patterns (as well evaluated at $r_{\mathrm{d}}=0.3 \mathrm{~nm}^{-1}$ ) is shown to discuss the effect of the overall decreasing diffraction intensity with $T$, i.e. the origin of the scaling factor in the definition of $I_{\mathrm{CO}}$. For this purpose, it is crucial to mention the order in which the diffraction patterns have been taken: After the acquisition at room temperature, the sample was cooled down and the zone axis was finetuned. Starting from $100 \mathrm{~K}$, the temperature was gradually increased and looped back to $100 \mathrm{~K}$ after the acquisition at $200 \mathrm{~K}$.

Next, it will now be shown that the black points correspond to the $\mathrm{CO}$ parameter. In general, there are five possible explanations for intensity vari- 


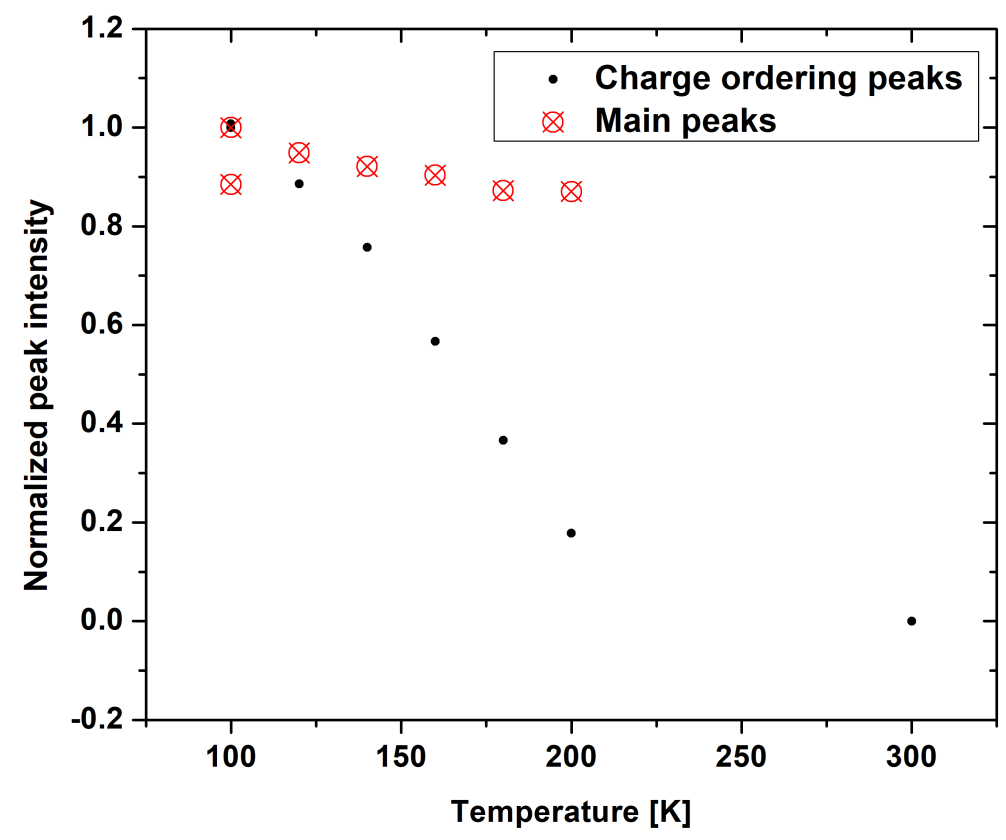

Figure 6.4: Temperature-dependent excess intensity due to charge ordering (black) as well as the averaged intensity of the main peaks (red) both evaluated at $r_{\mathrm{d}}=0.3 \mathrm{~nm}^{-1}$ and normalised to their maxima. Please note that two virtually identical points of the CO intensity coincide at $100 \mathrm{~K}$.

ations in the $\mathrm{CO}$ peaks extracted from DPs taken at different temperatures and times:

1. A change in the beam current: This effect can be ruled out on the relevant scale as the illumination condition was not changed during the experiment and the indicated value of the fluorescence screen with inserted SAED aperture was $72 \mathrm{pA}$ before and after the experiment.

2. A change in sample position: As each temperature point was stabilised for several minutes, the stage drift between positioning the SAED aperture in real space and finishing recording the DP $(<10 \mathrm{~s})$ can be estimated to be at maximum a few nanometers hence the main uncertainty is the manual positioning of the aperture with respect to 
the sample itself. Considering the related real space images yields positional deviations of a few $10 \mathrm{~nm}$. Given the SAED aperture diameter of $720 \mathrm{~nm}$, an exemplary shift of $30 \mathrm{~nm}$ corresponds to a $95 \%$ identical diffraction volume. Since the adjacent regions are not expected to exhibit fundamentally different diffraction patterns and the intensity changes in Fig. 6.4 are very systematic (while inaccurate positioning should lead to non-systematic behaviour), this effect seems to be negligible.

3. The temperature-dependent Debye-Waller factor: Considering the main peak intensity in Fig. 6.4 and comparing the two point at $100 \mathrm{~K}-$ please note that the lower point was taken after heating and thus closes the loop - indicates that the decrease in intensity is predominantly caused by temporal effects and the Debye-Waller effect is small.

4. A change in the relative orientation between the electron beam and the sample: In fact, a slight misalignment of the zone axis orientation is observed and presented in Fig. A.11 serving as a good explanation for the decreasing intensity in the main peaks in time. However, considering the two virtually identical points of the $\mathrm{CO}$ peak at $100 \mathrm{~K}$ in Fig. 6.4 suggests that the superstructure reflections are hardly influenced by the subtle zone axis misalignment. A possible explanation for this finding is a larger disorder in the superstructure compared to the main lattice, e.g. due to ordered and disordered domains as reported in [57]. As the zone axis change between the two acquisitions at $100 \mathrm{~K}$ is largest, no significant influence of the misalignment on the $\mathrm{CO}$ peak intensity (after background subtraction) is expected.

5. A change in the volume of $\mathrm{CO}$ ordered domains, i.e. the related ordering parameter: Since the points 1-4 can be ruled out as described above, only the ordering parameter of the $\mathrm{CO}$ phase itself serves as an explanation for the increase of the corresponding peak intensity observed in Fig. 6.4.

The rather smooth increase of the $\mathrm{CO}$ parameter fits well to the expected emergence of a two-phase region with coexisting ordered and disordered domains [57]. Extrapolating the three inner-most points linearly and evaluating the intersection with the temperature axis yields a $\mathrm{CO}$ onset at $T=218(4) \mathrm{K}$. Compared with the critical temperature in the bulk [163], this value is approximately $20 \mathrm{~K}$ lower indicating that epitaxial strain caused by the STO substrate resp. related defects destabilise the ordered phase. 
This finding is consistent with previously reported onsets of enhanced open circuit voltages in photovoltaic measurements [17] as well as hot polaron lifetimes [18]. However, whether and how much thin film effects influence the critical temperature depends on the underlying strain relaxation process and an unchanged $\mathrm{CO}$ temperature in a fully-relaxed Ruddlesdon-Popper PCMO thin film was recently demonstrated [27].

\subsection{Pseudo-Tetragonal Transition for $x=0.1$}

As already discussed in the previous chapters, an orthorhombic to pseudocubic transition at elevated temperatures is expected in the low-doping regime of bulk PCMO. In order to study whether such a transition occurs in epitaxially strained thin films as well, in-situ heating NBED exploiting the 4D-STEM developments presented above was performed and the corresponding results were published in the following paper [134] in Small Methods (doi.org/10.1002/smtd.202100464) - the author's contributions are given in the main text. All contents are reprinted in their original form with permission.

The findings prove that the structural transition exists in a $400 \mathrm{~nm}$ thick PCMO layer at a similar critical temperature compared with the bulk [20]. However, lacking a precise reference in the thin region of the TEM lamella, no quantitative comparison between in- and out-of-plane lattice parameters, i.e. $a / b$ resp. $c$, was possible such that the high-temperature phase could either be pseudo-tetragonal or pseudo-cubic. Thus, the more general former term is used herein. Importantly, observed hysteretic changes while heating and cooling the sample in high-vacuum could be avoided by using a lowpressure oxygen ambience, i.e. by employing an ETEM. Consequently, the paper presents not only the reversible structural but also irreversible phase transitions. 


\title{
Phase Transitions in a Perovskite Thin Film Studied by Environmental In Situ Heating Nano-Beam Electron Diffraction
}

\author{
Tobias Meyer, Birte Kressdorf, Vladimir Roddatis, Jörg Hoffmann, Christian Jooss, \\ and Michael Seibt**
}

The rich phase diagram of bulk $\operatorname{Pr}_{1-x} \mathrm{Ca}_{x} \mathrm{MnO}_{3}$ resulting in a high tunability of physical properties gives rise to various studies related to fundamental research as well as prospective applications of the material. Importantly, as a consequence of strong correlation effects, electronic and lattice degrees of freedom are vigorously coupled. Hence, it is debatable whether such bulk phase diagrams can be transferred to inherently strained epitaxial thin films. In this paper, the structural orthorhombic to pseudo-cubic transition for $x=0.1$ is studied in ion-beam sputtered thin films and differences to the respective bulk system are pointed out by employing in situ heating nanobeam electron diffraction to follow the temperature dependence of lattice constants. In addition, it is demonstrated that controlling the environment during heating, that is, preventing oxygen loss, is crucial in order to avoid irreversible structural changes, which is expected to be a general problem of compounds containing volatile elements under non-equilibrium conditions.

compounds contain volatile components, that is, elements in gaseous state at processing or even operating conditions. Prominent examples are $\mathrm{TiO}_{x}$ for improved solar cell contacts, ${ }^{[4,5]} \mathrm{ZnO}$ for ultraviolet light emitting diodes, ${ }^{[6,7]} \quad \mathrm{La}_{1-x} \mathrm{Sr}_{x} \mathrm{MnO}_{3}$ for solid oxide fuel cells, ${ }^{[8,9]} \mathrm{MgO}$ for magnetic tunneling junctions, ${ }^{[10-12]}$ $\mathrm{Li}_{1-x} \mathrm{CoO}_{2}$ for solid state batteries, ${ }^{[13-15]}$ or $\mathrm{Pr}_{1-x} \mathrm{Ca}_{x} \mathrm{MnO}_{3}$ (PCMO) for neuromorphic computing. ${ }^{[16-18]}$ As a general feature, the oxygen activity adjusted by ambient conditions delicately controls properties and reaction paths in such systems. Referring to the metal oxides related to solid state batteries and neuromorphic computing, such reaction paths and also applications rely on controllable, reversible phase transitions. Hence, typical non-equilibrium

\section{Introduction}

Current and future technologies increasingly exploit the unique characteristics of metal oxide thin films, ${ }^{[1-3]}$ where a plethora of physical properties can be tailored by temperature and composition dependent phase transitions. By definition, these

\author{
T. Meyer, ${ }^{[+]}$M. Seibt \\ 4th Institute of Physics - Solids and Nanostructures \\ University of Goettingen \\ Friedrich-Hund-Platz 1, 37077 Göttingen, Germany \\ E-mail: mseibt@gwdg.de \\ B. Kressdorf, V. Roddatis, ${ }^{[++]}$J. Hoffmann, C. Jooss \\ Institute of Materials Physics \\ University of Goettingen \\ Friedrich-Hund-Platz 1, 37077 Göttingen, Germany
}

The ORCID identification number(s) for the author(s) of this article can be found under https://doi.org/10.1002/smtd.202100464.

(c) 2021 The Authors. Small Methods published by Wiley-VCH GmbH. This is an open access article under the terms of the Creative Commons Attribution-NonCommercial-NoDerivs License, which permits use and distribution in any medium, provided the original work is properly cited, the use is non-commercial and no modifications or adaptations are made.

[+]Present address: Institute of Materials Physics, University of Goettingen, Friedrich-Hund-Platz 1, 37077 Göttingen, Germany

$\left[{ }^{++}\right]$Present address: German Research Centre for Geosciences GFZ, Telegrafenberg Potsdam 14473, Germany

DOI: $10.1002 / \mathrm{smtd} .202100464$ operation and processing conditions offer the route to steer the latter, ${ }^{[19-21]}$ but also involve the risk of undesired reaction paths. In addition, the incorporation in structured devices raises the question whether and how phase diagrams obtained on bulk materials can be transferred to the nanomaterial counterparts.

Calcium doped $\mathrm{PrMnO}_{3}\left(\operatorname{Pr}_{1-x} \mathrm{Ca}_{x} \mathrm{MnO}_{3}\right.$, PCMO) is a prominent representative of strongly correlated manganites discussed in the context of neuromorphic computing, but also related to third generation photvoltaics ${ }^{[22-24]}$ and catalysis. ${ }^{[25-28]}$ Due to strong correlations, the system - as many other strongly correlated materials ${ }^{[29]}$ - has a rich phase diagram with several ordered phases showing remarkable properties. ${ }^{[30-32]}$ Various studies have focused on magnetic ordering at low temperature which is well understood. ${ }^{[33-35]}$ In addition, phase transitions at higher temperature of several hundred degrees Celsius have been revealed by neutron and X-ray diffraction experiments on PCMO powders for low Ca concentrations. ${ }^{[33,36]}$ They show the transition from an orthorhombic to pseudo-cubic phase with small but still finite deviations of the lattice parameters from an ideal cubic structure. These changes are related to a reduction of the cooperative JahnTeller (JT) distortion as well as the alternating tilt of neighbouring $\mathrm{MnO}_{6}$ octahedra in the high-temperature phase. Still, there is no direct evidence of orbital ordering driving the phase transition and latest results suggest that the orbital ordering above room temperature is induced by octahedral tilting. ${ }^{[3]}$

A major unsolved problem, however, is whether and how such data can be transferred to epitaxial thin films, which are the typical basis for technological application. More generally, this is 
a

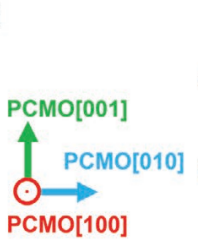

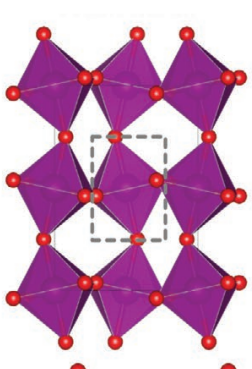

b
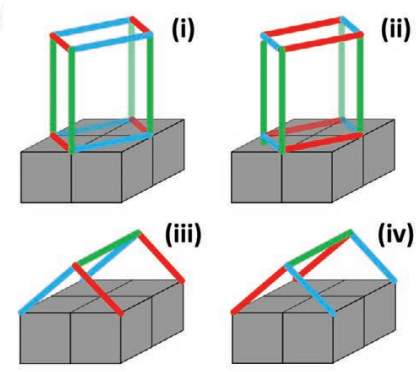

(v)

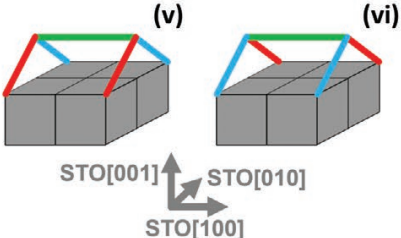

C

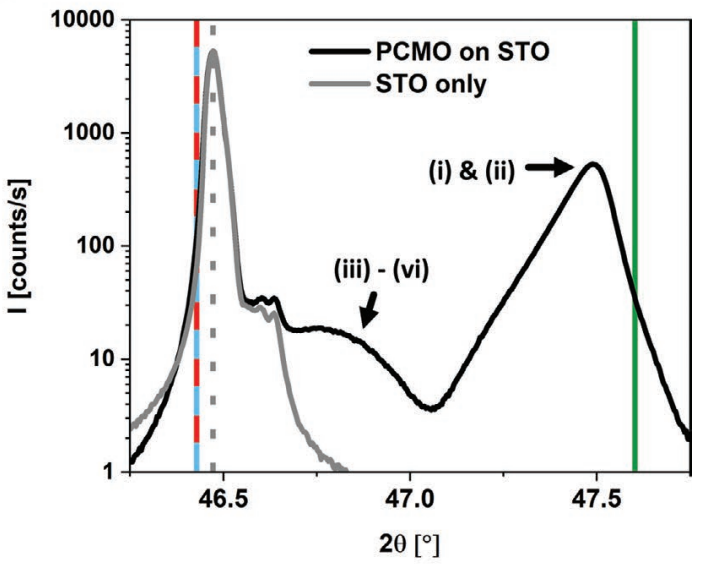

Figure 1. a) Tilt pattern of the $\mathrm{MnO}_{6}$ octahedra in PCMO $\left(a^{-} a^{-} c^{+}\right.$in Glazer notation $\left.{ }^{[43]}\right)$ with $\mathrm{Mn}$ and $\mathrm{O}$ in purple and red, respectively. For simplicity, $\mathrm{Pr}$ and $\mathrm{Ca}$ are not shown. The ideal, cubic perovskite unit cell in the absence of tilt and distortion is indicated by dashed gray rectangles. b) Possible epitaxial growth relations of PCMO on cubic STO (gray cubes) using the same colour code for the PCMO lattice constants as in (a). c) XRD intensity obtained by a $\Theta-2 \Theta$ scan of a macroscopic, $400 \mathrm{~nm}$ thick epitaxial thin film on STO (black) as well as a pristine STO substrate (gray) at room temperature with the bulk values of PCMO(004) (solid green), PCMO(220) (dashed red-cyan), and STO(200) (dotted gray) ${ }^{[34,44]}$ marked.

equivalent to the question whether bulk phase diagrams remain unchanged in thin film systems. In fact, particularly in the context of transition metal oxides, various reports exist about tunable physical properties by epitaxial strain and oxygen vacancies possibly affecting phase transition temperatures as well. ${ }^{[38-41]}$

In this work, we investigate phase transitions at high temperature in PCMO $(x=0.1)$ grown as a $400 \mathrm{~nm}$ thick epitaxial film on a $\mathrm{SrTiO}_{3}$ (STO) substrate. In order to extract temperaturedependent lattice parameters we use nano-beam electron diffraction (NBED) in a 4D scanning transmission electron microscopy (4D-STEM) mode. ${ }^{[42]}$ Hence, individual domains of the typically nanotwinned films can be addressed in contrast to macroscopic X-ray or neutron diffraction studies. Furthermore, during the in situ heating experiments, the environmental capabilities of the electron microscope are used to compare reaction paths in ultrahigh vacuum (UHV) and $10 \mathrm{~Pa}$ oxygen partial pressure ambient conditions. In the latter case, a reversible orthorhombic to pseudo-cubic phase transition is observed, while in the former case, an irreversible phase transition associated with the appearance of superstructure reflections occurs, which completely suppresses the orthorhombic to pseudo-cubic phase transition. These results will be discussed in terms of oxygen vacancies whose formation is controlled by the ambient conditions.

\section{Results and Discussion}

Prior to considering the temperature dependence of the given lattice parameters, a brief overview of the crystal structure of PCMO as well as the thin film epitaxy and heterogeneity shall be given. Figure 1a illustrates the expected tilt pattern of corner-sharing $\mathrm{MnO}_{6}$ octahedra in PCMO $\left(a^{-} a^{-} c^{+}\right.$in Glazer notation ${ }^{[43]}$ ) leading to an orthorhombic Pbnm unit cell. For the sake of simplicity, A-site atoms, that is, Pr and Ca are not shown. Compared with the cubic unit cell of an ideal perovskite like STO containing only one octahedron which is neither tilted nor distorted, the lattice vector PCMO[001] is approx. two times larger due to the tilt alternation. Similarly, PCMO[100] and PCMO[010] are approx. $\sqrt{2}$ times larger and rotated by approx. $45^{\circ}$. Consequently, six epitaxial growth relations of PCMO on a cubic STO substrate (with STO(001) surface termination) exist as illustrated in Figure 1b. The moduli of the corresponding bulk lattice vectors at room temperature are given in Table 1. Importantly, a change of the space group of PCMO is not expected in the pseudo-cubic phase, in which the octahedral tilt is strongly reduced but still finite. ${ }^{[3,36]}$

The $\Theta-2 \Theta$ X-ray diffraction (XRD) scan of the epitaxial thin film presented in Figure 1c shows a clear peak close to the PCMO(004) bulk value (solid green line) and only subtle differences compared with the reference scan of a pristine substrate in the range of the $\mathrm{PCMO}(220)$ bulk value (dashed red-cyan line). Thus, the XRD data suggests predominant growth relations (i) and (ii) and the position of the corresponding peak yields $+0.2 \%$ tensile strain of the out-of-plane PCMO[001] lattice parameter being consistent with previous reports about significant deviations from bulk properties in relatively thick PCMO films due to both misfit as well as preparation related strain that is induced by point defects and variations in octahedral tilt. ${ }^{[45,46]}$

The XRD findings as well as high epitaxial growth quality is confirmed by the transmission electron microscopy (TEM) results, which were obtained with an STO[110] zone axis alignment throughout the entire manuscript: As shown in Figure 2

Table 1. Room temperature values of the moduli of relevant lattice vectors.

\begin{tabular}{lcccc}
\hline Lattice vector & PCMO[100] & PCMO[010] & PCMO[001] & STO[100] \\
\hline Modulus $[\AA ̊]$ & $5.442^{[34]}$ & $5.617^{[34]}$ & $7.635^{[34]}$ & $3.905^{[44]}$ \\
\hline
\end{tabular}



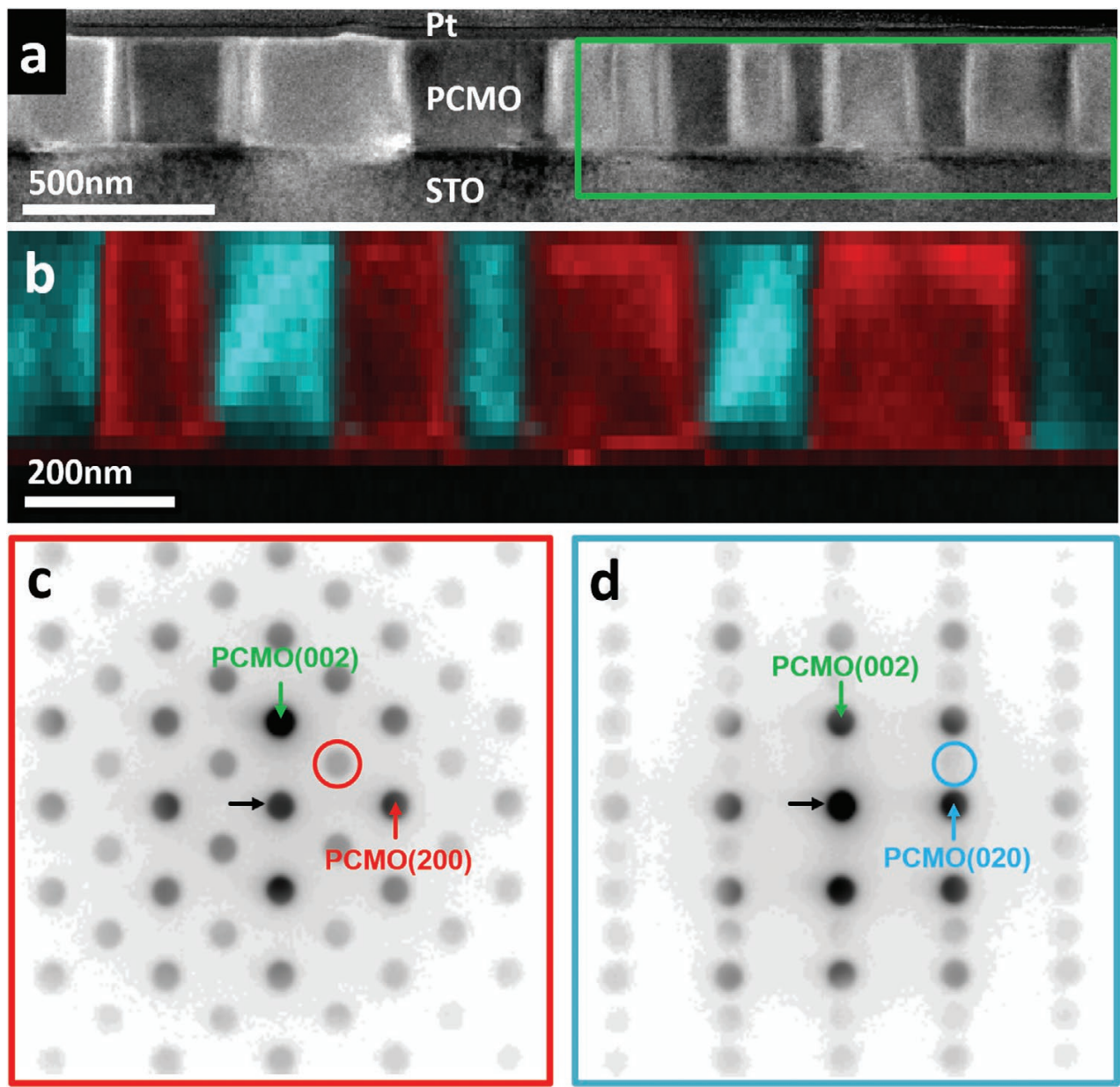

Figure 2. a) Annular dark field (ADF)-STEM overview at room temperature with the scanning area marked with the green rectangle. b) Domain structure of the PCMO thin film visualized by virtual dark-field images created by integrating the intensity of the PCMO(101) (red circle) and PCMO(021) (cyan circle) reflection, respectively. The STO[110] zone axis oriented substrate appears dark since neither of these reflections is allowed. (c) and (d) NBED patterns corresponding to the red and cyan parts of the PCMO film with PCMO[010] and PCMO[100] zone axis orientation, respectively. The direct beam is marked with black arrows.

and expected for the relations (i) and (ii), the corresponding zone axis in the film is found to be either PCMO[100] or PCMO[010], that is, PCMO[001] is oriented out-of-plane in both cases. In fact, the in-plane orientation flips quite regularly on a few hundred nanometer scale and the resulting domains extend through the entire film as clearly visualized by the 4D-STEM analysis shown in Figure $2 \mathrm{~b}-\mathrm{d}$. This reflects a nanotwinned state of the film as it is often observed in orthorhombic systems. ${ }^{[47,48]}$

The temperature dependent lattice parameters obtained in the TEM and averaged over the upper half of the scanning field in Figure 2 are shown in Figure 3. The error bars represent the standard deviation in the averaged range. Noteworthy, the initial value of the out-of-plane lattice parameter, that is, PCMO[001], is consistent with the macroscopic XRD measurement presented in Figure 1c. Upon heating, the in-plane parameter, including contributions from PCMO[100] and PCMO[010], decreases with higher temperature. In contrast, PCMO[001] increases and is strongly hysteretic. The latter indicates an irreversible change of the TEM lamella during heating which is confirmed by a change in the crystal symmetry: Figure $4 \mathrm{a}, \mathrm{b}$ shows series of
NBED patterns of domains with PCMO[100] and PCMO[010] zone axis orientation, respectively. While in the former case a $\mathrm{PCMO}\left(0 \frac{1}{2} 0\right)$ superreflection emerges at $800 \mathrm{~K}$, the PCMO(101) reflection vanishes in the latter. Both effects persist at lower temperatures after heating and in addition, the initially extinct PCMO(100) reflection shows up in domains with PCMO[010] zone axis orientation. The appearance of superstructures, although not exclusively occurring in one direction, has been reported before in the context of catalytical studies of PCMO $(x=0.36)$ and was attributed to oxygen vacancy ordering. ${ }^{[27]}$ The circumstance that ordering is only observed along PCMO[010] might be related to the results in ref. [50] for $x=1$ showing that in the presence of strain certain lattice sites are preferred for oxygen vacancy formation. Recalling the structural sketch presented at the bottom of Figure 1a, the emerging superstructure could be explained by oxygen vacancies occurring only at the oxygen site highlighted by a black circle but not at the site highlighted by a green circles. However, other configurations of oxygen vacancies are consistent with the observed superstructure along $\mathrm{PCMO}[010]$ and the change in extinction rules being strongly linked to the tilt pattern of neighbouring $\mathrm{MnO}_{6}$ 


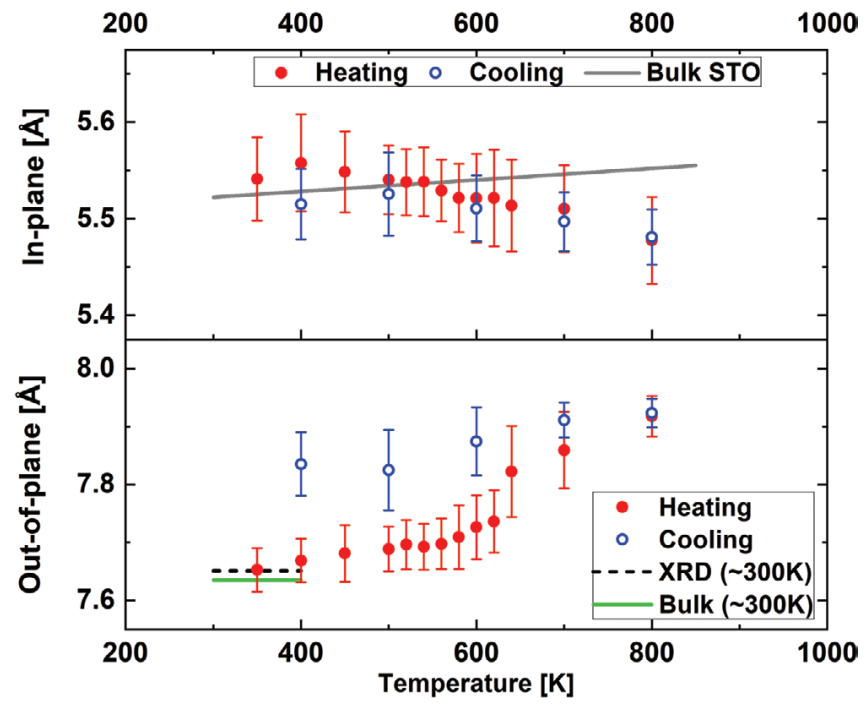

Figure 3. Temperature-dependent in and out-of-plane lattice parameters obtained under UHV conditions averaged over the upper half of the scanning range shown in Figure 2 as well as the room temperature values of bulk PCMO[001] ${ }^{[34]}$ (solid green) and as obtained on the film by XRD (dashed black). The temperature-dependent corresponding lattice plane distance of bulk STO ${ }^{[49]}$ is plotted as a solid gray line. The error bars correspond to the standard deviations in the averaged range. While the averaged in-plane parameter decreases slightly in temperature, the out-of-plane parameter shows a strong increase as well as obvious hysteretic behavior.

octahedra in stoichiometric perovskites ${ }^{[51]}$ - suggests additional atomic reconfigurations. Noteworthy, the hysteretic increase of the out-of-plane lattice parameter in Figure 3 is qualitatively consistent with previously reported lattice expansion due to oxygen deficiency. ${ }^{[35]}$

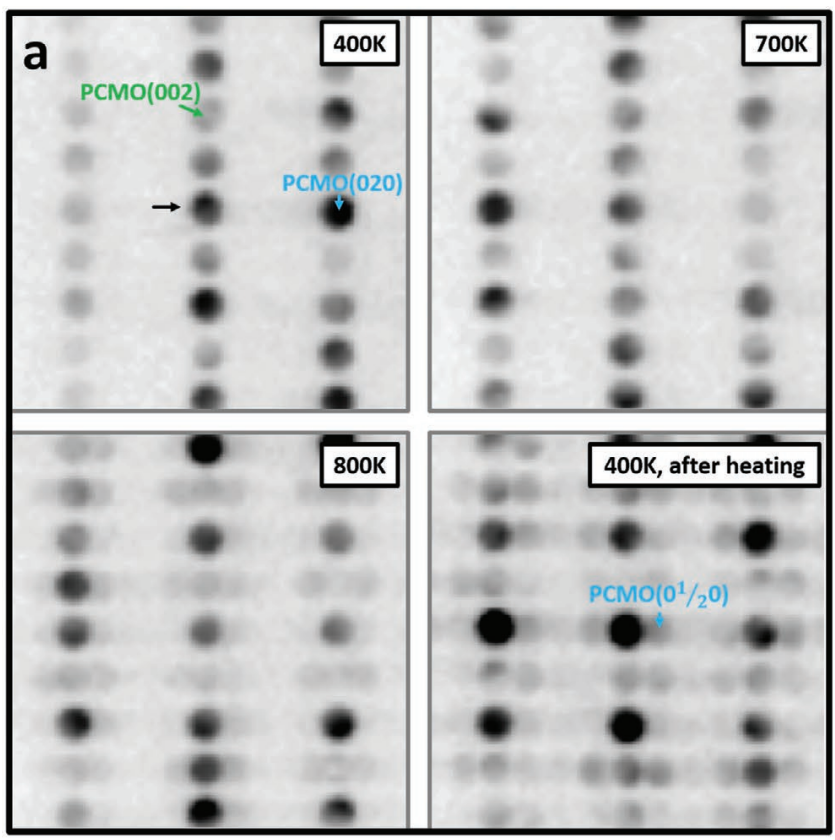

In order to suppress oxygen vacancy formation, an analogous experiment was conducted facilitating the environmental TEM (ETEM) gas-inlet capabilities and using an oxygen partial pressure of $10 \mathrm{~Pa}$. The resulting lattice parameters obtained in the gaseous environment are shown in Figure 5. As a matter of fact, PCMO[001] still increases strongly with temperature but in contrast to the experiment in high-vacuum no significant hysteresis is visible. Furthermore, neither a $\operatorname{PCMO}\left(0 \frac{1}{2} 0\right)$ superreflection nor a change in the extinction rules was observed in the gaseous environment confirming oxygen vacancy formation to be directly linked to the previous irreversible changes.

A striking observation is the sudden jump of the averaged in-plane parameter at $600 \mathrm{~K}$ during heating, which is not reverted during cooling. As a result, the averaged in-plane parameter approaches the lattice parameter of the substrate at that temperature proving its effect on the strain state of the PCMO. Bearing in mind that the PCMO films have been postgrowth annealed at $1173 \mathrm{~K}$ this jump has to be attributed to the reduced thickness $(\simeq 100 \mathrm{~nm})$ of the TEM foil along the electron beam direction, which permits further change of epitaxial strain. Following the curve after the jump reveals a reversible decrease of the averaged in-plane parameter at high temperatures. In fact, the black data points in Figure 5 (with the zone axis fine-adjusted at $900 \mathrm{~K}$ ), which were acquired after the first heating cycle (with the zone axis fine-adjusted at $300 \mathrm{~K}$ ), demonstrate that no significant irreversible changes are observed in the second cycle as well as that mistilt, for example, due to membrane bulging, does not hamper the interpretation of the presented lattice parameters.

To disentangle the behavior of the so far averaged in-plane lattice parameters $\mathrm{PCMO}[100]$ and $\mathrm{PCMO}[010]$, the temperature dependent histograms of detected in-plane lattice

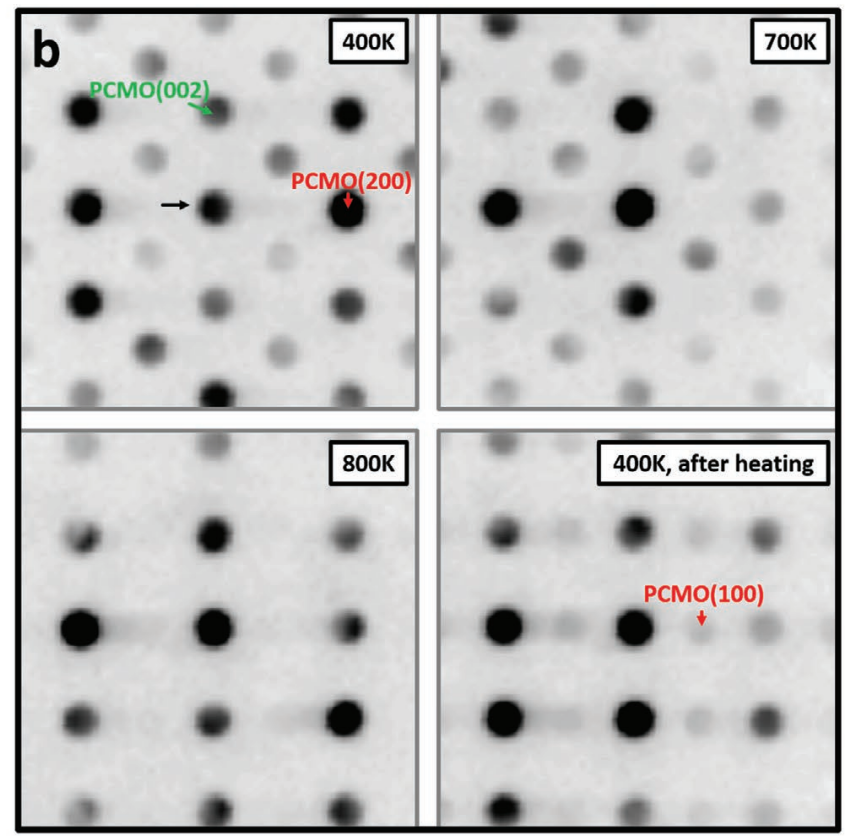

Figure 4. Temperature series of NBED patterns obtained under UHV conditions. a) In domains with PCMO[100] zone axis, a PCMO $\left(0 \frac{1}{2} 0\right)$ superreflection is observed first at $T=800 \mathrm{~K}$ which becomes increasingly obvious in time and persists at low temperatures after heating. b) In domains with PCMO[010] zone axis, the PCMO(101) reflection vanishes at $800 \mathrm{~K}$ and during the course of the experiment the initially extinct PCMO(100) reflection emerges. 


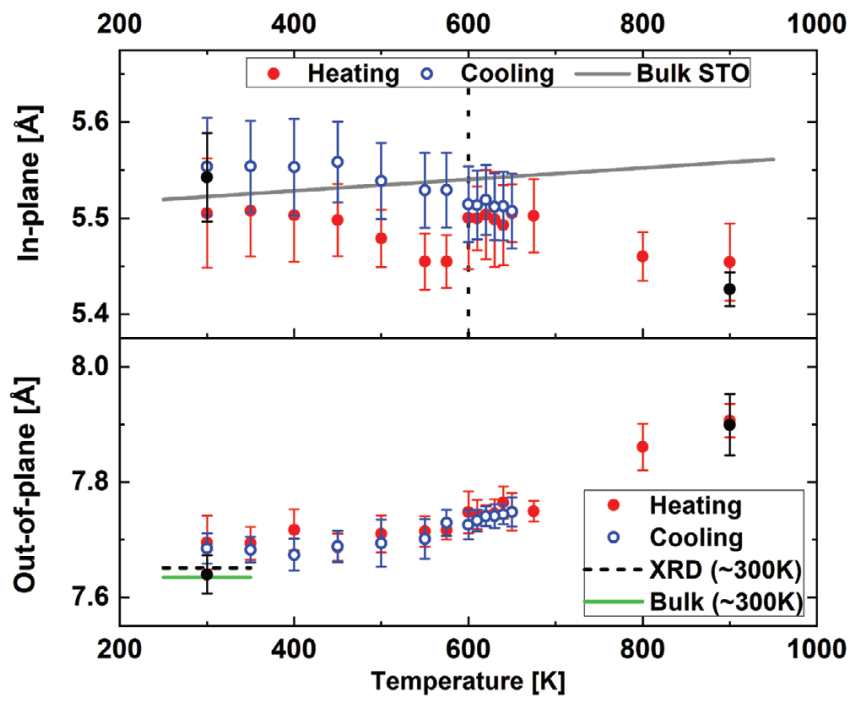

Figure 5. Temperature-dependent in and out-of-plane lattice parameters obtained in a $10 \mathrm{~Pa}$ oxygen environment as well as the room temperature values of bulk PCMO[001] ${ }^{[34]}$ (solid green) and as obtained on the film by XRD (dashed black). The temperature-dependent corresponding lattice plane distance of bulk STO ${ }^{[49]}$ is plotted as a solid gray line. The error bars correspond to the standard deviations in the averaged range. While the averaged in-plane parameter decreases in temperature and exhibits a sudden jump during heating at $600 \mathrm{~K}$, the out-of-plane para parameter shows a strong increase. The black data points were acquired after the first heating and cooling cycle with the zone axis fine-adjusted at $900 \mathrm{~K}-$ in contrast to the fine-adjustment at $300 \mathrm{~K}$ during the first cycle-in order to assess the effect of mistilt due to bulging of the heating chip. constants during heating and cooling are shown in Figure 6a and Figure 6b, respectively. Please note that the histograms above $T=650 \mathrm{~K}$ in Figure $6 \mathrm{~b}$ are re-plotted from (a). Generally, before and after heating, a bimodal distribution is observed at low temperatures and the two distinct peaks with lower and higher lattice parameter correspond to domains with PCMO[010] and PCMO[100] zone axis orientation, respectively. As the temperature is raised, the two peaks start to merge and a tetragonally-strained pseudo-cubic phase forms. It should be noted that the sudden jump during heating at $600 \mathrm{~K}$ discussed above occurs right at the pseudo-cubic transition in Figure $\mathbf{6 a}$ suggesting that isotropic lattice parameters are beneficial for this type of rearrangement. The temperature-dependent corresponding lattice spacing of bulk STO (solid gray line) ${ }^{[49]}$ indicates an increased adaptation of the in-plane lattice parameters of the film to the substrate at $600 \mathrm{~K}$. Furthermore, the arrows marking the room temperature bulk values of PCMO[100] (red) and PCMO[010] (cyan) ${ }^{[34]}$ reveal increased tensile strain of PCMO $[100]$ and decreased compressive strain of PCMO[010] at room temperature after heating. This again proves the epitaxial strain maintained by the substrate regardless of the thin foil effect discussed above.

Despite the mentioned modifications of the strain state, no significant changes in size or position of the nanotwinned domains are observed: As presented in Figure 7a,b, the mesoscopic structure of nanotwinned domains is recovered after heating. Furthermore, it should be noted, that the Moiré patterns arising from the lattice resolution of the ADF-HRSTEM images in Figure 7d,e obtained at small magnification are a a

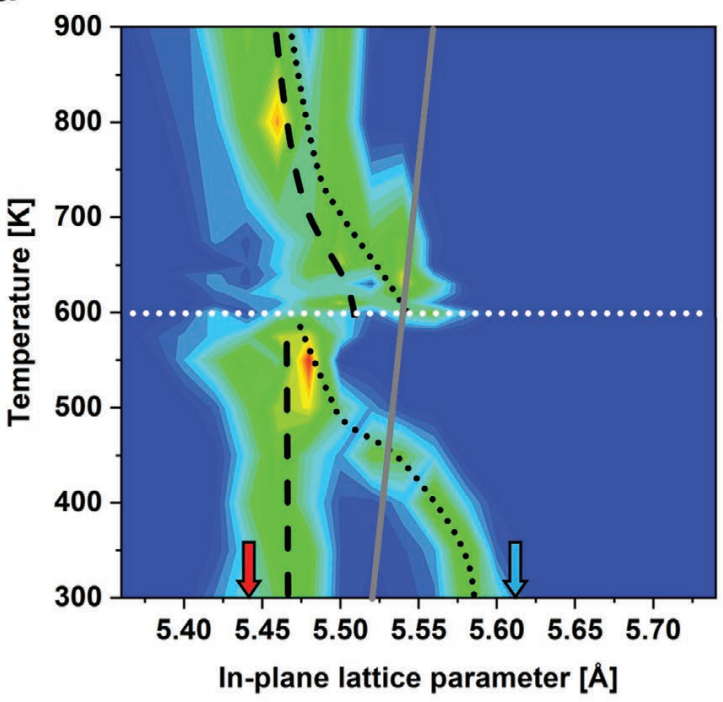

b

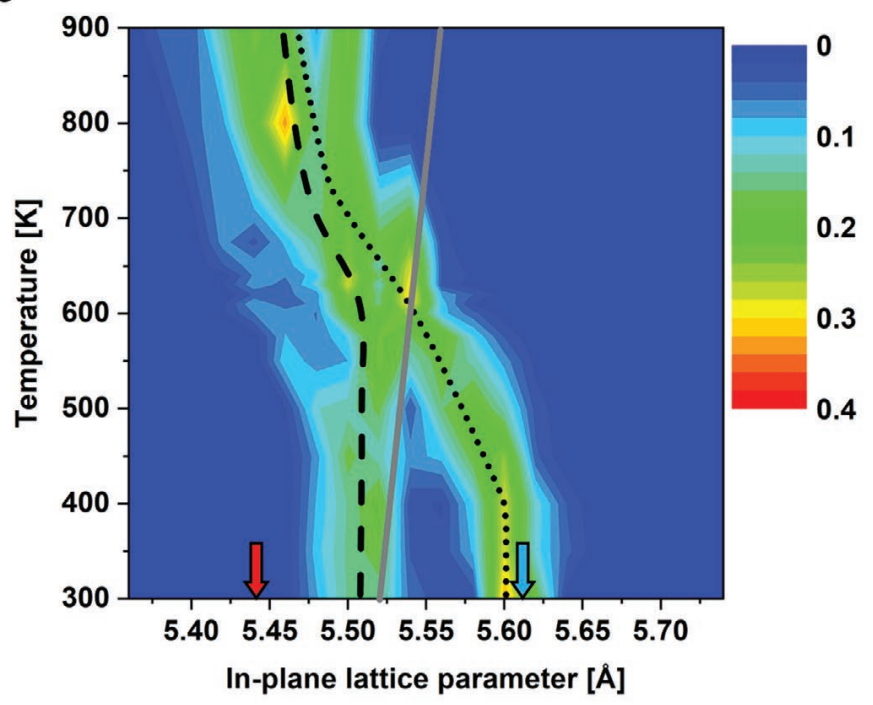

Figure 6. Contour plot of the temperature-dependent histograms of the in-plane lattice parameter obtained in a 10 Pa oxygen environment in an approximately $600 \mathrm{~nm} \times 150 \mathrm{~nm}$ scanning area at $150 \mathrm{~nm}$ distance from the substrate. The colors represent the relative frequency and the dashed and dotted black lines are a guide to the eye to follow PCMO[100] and PCMO[010]. (a) includes the data recorded during heating and shows a sudden jump at $600 \mathrm{~K}$. (b) shows the data recorded during cooling (except for $T>650 \mathrm{~K}$ as only heating points exist) in which no jump is present. The splitting of PCMO[100] and PCMO[010] recurs after heating. The arrows mark the bulk values of PCMO[100] (red) as well as PCMO[010] (cyan) at room temperature, and the gray line indicates the temperature-dependent corresponding lattice plane spacing of bulk STO. ${ }^{[34,44]}$ Potential systematic errors due to assuming an unstrained substrate as a reference for quantification are estimated to be $\approx 3$ pm (see Experimental Section). 

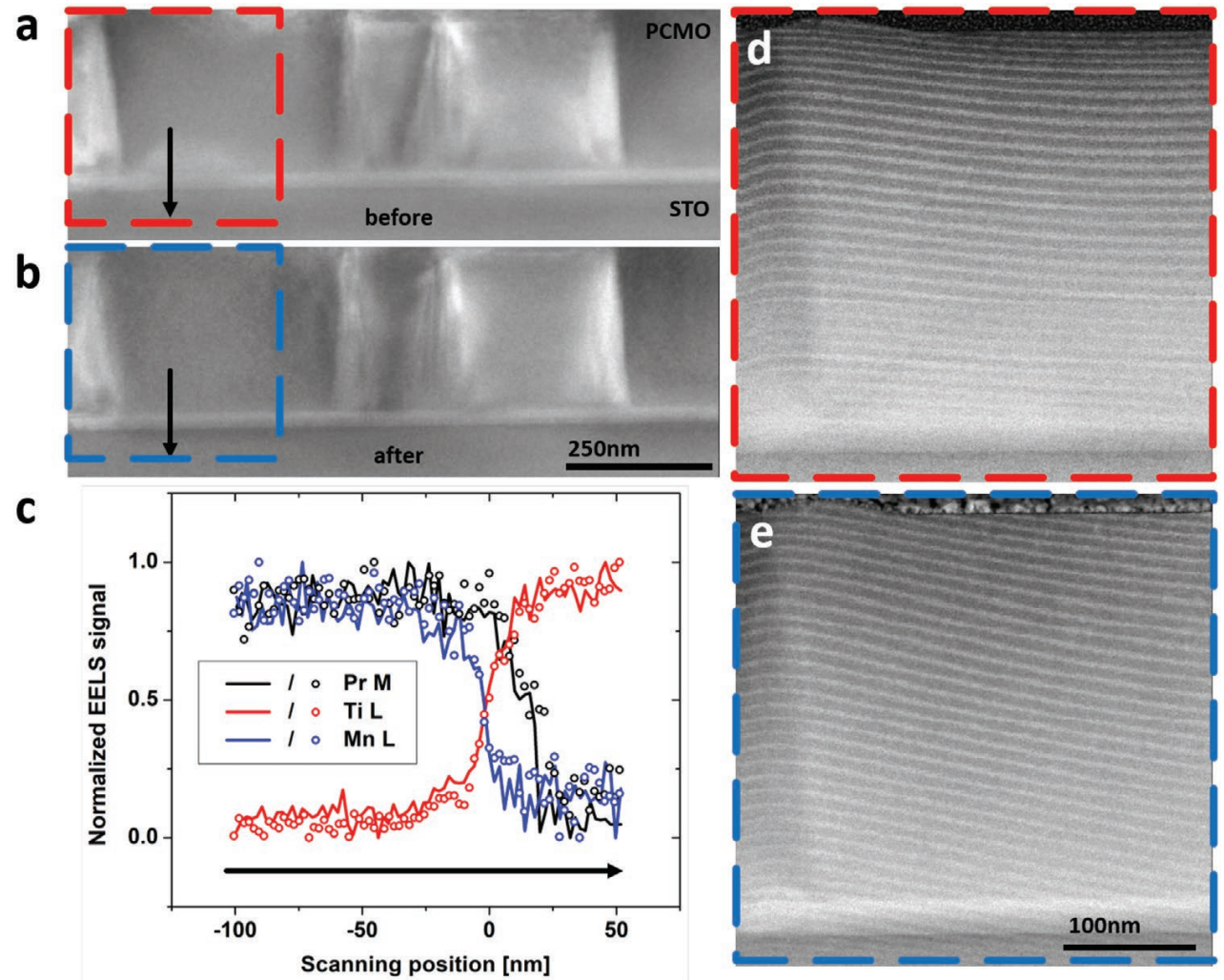

Figure 7. ADF-STEM overview of the nanotwinned domains structure under NBED conditions at room temperature a) before and b) after in situ heating in a $10 \mathrm{~Pa}$ oxygen environment showing no significant changes in the domain size or position. c) EELS signal of the Pr M- (black), Ti L- (red), and Mn L-edge (blue) normalized by their maxima showing chemical interdiffusion of $\mathrm{Ti}$ and $\mathrm{Mn}$ in the range of the bright interface layer visible in (a) and (b). Lines and open circles correspond to the state before and after heating. (d) and (e) ADF-HRSTEM images of the marked areas in (a) and (b). The bright fringes reflect Moiré patterns of the scanning array with the crystal structure proving a sufficiently focused probe to achieve lattice resolutions.

sensitive test for irreversible changes ruling out crack formation during the rearrangement. In addition, electron energy-loss spectroscopy (EELS) signals of the Pr M- (black), Ti L- (red), and Mn L-edge (blue) are provided in Figure 7c revealing chemical interdiffusion of $\mathrm{Ti}$ and $\mathrm{Mn}$ in the interface layer apparent by a bright contrast in Figure 7a,b, which is consistent with previous reports. ${ }^{[52]}$ It should be noted that comparing the data before (lines) and after (open circles) heating excludes significant changes of the interdiffusion layer. Consequently, changes of the domain boundary nanostructure or of preparation-related point defect concentrations and variations in octahedral tilt are possible explanations for the structural modification.

Although thin film strain relaxation phenomena are not in the focus of this study, some insight into the role of domain a

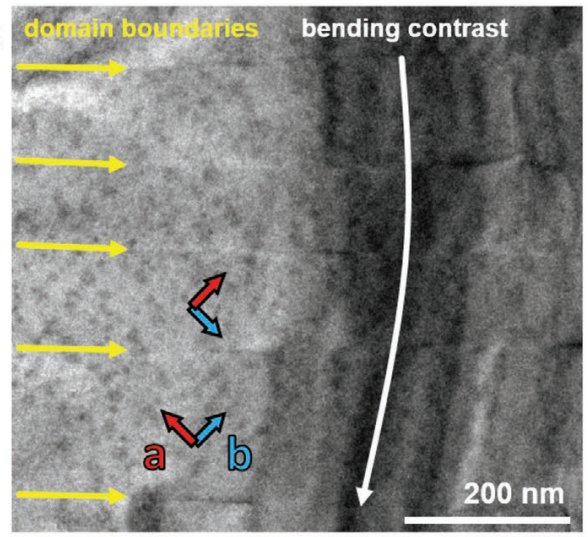

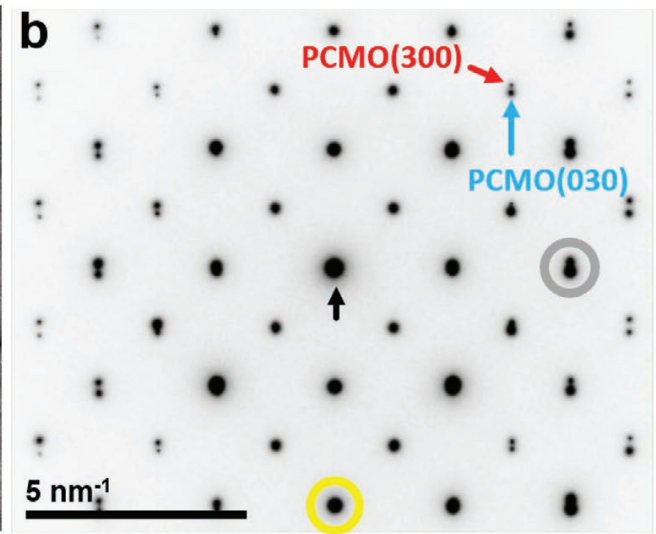

Figure 8. a) Plan-view BF-TEM image with several horizontally aligned nanotwin domain boundaries indicated by yellow arrows. The dark vertical contrast is caused by lamella bending. b) SAED pattern of the region shown in (a). The direct beam as well as the PCMO(300) and PCMO(030) reflections - corresponding to alternating domain orientations — are highlighted by a black, red and blue arrow, respectively. 
boundaries for the rather complex strain-stress state of the nanotwinned thin films is provided by Figure 8a,b, showing a plan-view TEM image and a selective area electron diffraction (SAED) pattern, respectively. There are two important observations, that is, (i) the domain boundaries indicated by yellow arrows in (a) tend to follow PCMO(110) and PCMO(110) lattice planes, and (ii) domains are strongly elongated in the horizontal and closely spaced in the vertical direction (note, that regions with the same arrangement but tilted by $90^{\circ}$ are also observed but not shown here). As a third feature, lattice planes of the nanotwins parallel to the boundaries are aligned to each other while the planes perpendicular to the boundaries are canted as can be inferred from the split and overlapping peaks marked by a gray and yellow circle in Figure $8 \mathrm{~b}$. As the cross-sectional lamellas used in the in situ experiment where extracted along the diagonal direction in Figure 8a (yielding alternating $\mathrm{PCMO}[100]$ and $\mathrm{PCMO}[010]$ zone axis orientations), the elongated domains are cut at an angle of $45^{\circ}$. Thus, small rotations of the PCMO(110) and PCMO(110) lattice planes coinciding at domain boundaries-accompanied by defect formation-should be more favourable in the thin TEM foil compared with the macroscopic counterpart. Hence, the observed rearrangement at $600 \mathrm{~K}$ can tentatively be attributed to these small relative rotations in combination with expansion of the film along the electron propagation direction feasible in the TEM lamella. Notwithstanding these rather complex relaxation phenomena resulting from the nanotwinned microstructure, the orthorhombic to pseudo-cubic phase transition is observed in the thin film if heated up in an oxygen environment.

Finally, the results obtained in oxygen environment after the rearrangement shall be compared to bulk measurements. As described in ref. [34], a pseudo-cubic transition is expected in the bulk for $x=0.1$ at $\approx 675 \mathrm{~K}$. Our findings show that the transition occurs at a slightly lower temperature in the thin film, that is, at $\approx 600 \mathrm{~K}$. Unfortunately, since no temperaturedependent lattice constants are published at this calcium concentration, we can compare the trends only qualitatively to the existing data obtained in the bulk for $x=0 \cdot \cdot^{[33,36]}$ Here, due to the pseudo-cubic transition at $1050 \mathrm{~K}, \mathrm{PCMO}[001]$ increases by $3.8 \%$, PCMO[010] decreases by $3.4 \%$, and PCMO[100] increases by $1.5 \%$. Since the initial anisotropy at room temperature is weaker for $x=0.1$, it is to be expected that theses changes are smaller due to the increased calcium content matching very well the observed increase of $\mathrm{PCMO}[001]$ by $\approx 3.0 \%$ in Figure 5 and the decrease of $\mathrm{PCMO}[010]$ by $\approx 2.5 \%$ in Figure $6 \mathrm{~b}$. On the contrary, PCMO[100] behaves qualitatively differently as it decreases with temperature. Even though no direct comparison to the bulk can be drawn for $x=0.1$, it seems very likely that this effect is rather caused by strain than by the finite value of $x$. We justify this claim with the fact that the increase of $\mathrm{PCMO}[100]$ is a fingerprint of the reduced $\mathrm{MnO}_{6}$ tilting angle having a stronger effect than the reduction of its JT distortion (which would in turn lead to an expansion in this direction). As the JT distortion is almost $50 \%$ weaker for $x=0.1$ at room temperature $^{[34]}$ and the tolerance factor, that is, the main driving force for octahedral tilts changes only by a few percent, ${ }^{[33]}$ we do not expect a reverted behavior due to the increased calcium content and attribute the reverted trend to the anisotropic nanotwinned strain state.

\section{Conclusion}

In summary, nano-beam electron diffraction in combination with in situ heating in an environmental transmission electron microscope was used to study phase transitions in $\mathrm{Pr}_{1-x} \mathrm{Ca}_{x} \mathrm{MnO}_{3}$ thin films epitaxially grown on $\mathrm{SrTiO}_{3}$ in the low-doping regime, that is, for a Ca concentration of 10 at\%. The oxygen activity was varied by the oxygen partial pressure during the in situ experiment leading to two distinctly different routes followed by the system.

On the one hand, UHV conditions accompanied by oxygen loss and hence formation of oxygen vacancies in the thin film material. From the appearance of a $\operatorname{PCMO}\left(0 \frac{1}{2} 0\right)$ superstructure accompanied by a change in the extinction rules as shown in Figures 3 and 4 , vacancy ordering can be concluded which persists after cooling to room temperature indicating a thermodynamically stable oxygendeficient PCMO. We note here, that the observed anisotropy might be related to misfit strain resulting in an anisotropic oxygen vacancy formation enthalpy and formerly equivalent sites where oxygen vacancies could occur alternatingly along the PCMO[010] direction are marked by a black and green circle in Figure 1a.

On the other hand, $10 \mathrm{~Pa}$ oxygen partial pressure in the gaseous ambient inside the microscope column sufficiently reduces the oxygen loss and hence completely suppresses the hysteretic behavior of the out-of-plane lattice parameter PCMO[001], the change in extinction rules, as well as the superstructure formation. As a consequence, the clear detection of the reversible orthorhombic to pseudo-cubic phase transformation is possible with a slightly decreased critical temperature of $\approx 600 \mathrm{~K}$ when compared to the bulk counterpart. ${ }^{[34]}$ In addition, a reverted behavior of the in-plane lattice parameter PCMO[100] was observed which was attributed to strain due to the cubic substrate and the mosaiclike microstructure of the films. It is quite interesting to note, that the irreversible relaxation occuring at about $600 \mathrm{~K}$ during the first heating ramp coincides with the pseudo-cubic transition shown in Figure 6a, that is, when the in-plane lattice parameters $\mathrm{PCMO}[100]$ and PCMO[010] are approaching lifting the strain relaxation due to the alternating twinned domains.

Finally, let us mention some implications of the results reported here. First of all, the study of phase transitions in thin film transition metal oxide perovskites by means of in situ electron nanobeam diffraction in 4D-STEM mode is an approach with several advantages over bulk diffraction studies. We have clearly demonstrated that controlling the oxygen activity by ambient conditions is not only a prerequisite for reliable in situ heating diffraction studies, but also possible in an environmental transmission electron microscope. This important conclusion can be generalized to other systems containing volatile components like nitrides and hydrides although details will depend on the properties of the specific system.

\section{Experimental Section}

A $400 \mathrm{~nm}$ thick PCMO film was grown on a commercial STO substrate with STO(100) surface termination by ion beam sputtering from a single target of composition $(x=0.1)$. Deposition parameters were: $p_{\text {Ar }}=3 \times 10^{-2} \mathrm{~Pa}$ (beam neutralizer), $p_{\mathrm{Xe}}=1 \times 10^{-2} \mathrm{~Pa}$ (sputter gas), and $p_{\mathrm{O}}=1.6 \times 10^{-2} \mathrm{~Pa}$ (film oxidation). The deposition temperature was set to $820^{\circ} \mathrm{C}$ using a Tectra boron nitride heater which results in $\approx 720^{\circ} \mathrm{C}$ at 
Table 2. Windows used for power-law background subtraction as well as signal integration of EELS data for all relevant edges.

\begin{tabular}{lcc}
\hline Edge & Background window $[\mathrm{eV}]$ & Signal window $[\mathrm{eV}]$ \\
\hline $\operatorname{Pr} M$ & $870-920$ & $920-1020$ \\
Ti L & $395-345$ & $445-495$ \\
$\mathrm{Mn} \mathrm{L}$ & $580-630$ & $630-680$ \\
\hline
\end{tabular}

the substrate surface. Subsequently, the film was annealed in air for $20 \mathrm{~h}$ at $900{ }^{\circ} \mathrm{C}$ with a ramping speed of $100{ }^{\circ} \mathrm{C}$ per hour.

TEM lamellas were extracted using an FEI Nova NanoLab Dual Beam focused ion beam and mounted on first generation four-contact DENSsolutions heating chips for the cross-sectional in situ experiment. For plan-view investigation, the preparation procedure described in ref. [53] was used. In both cases, the final thinning step was done using an acceleration voltage of $5 \mathrm{kV}$. TEM experiments were conducted in an FEI Titan ETEM G2 80-300 operated at $300 \mathrm{kV}$ using a DENSsolutions Lightning D7+ holder and a semi-convergence angle of $0.8 \mathrm{mrad}$ (NBED) and $10 \mathrm{mrad}$ (HR-STEM), respectively. ADF-STEM images were acquired with an inner and outer collection semi-angle of $46.8 \mathrm{mrad}$ and 200 mrad, respectively. EELS data was acquired with a Gatan Quantum 965 ER using a dispersion of $1 \mathrm{eV} /$ channel under HR-STEM conditions and analyzed with HyperSpy ${ }^{[54]}$ using the windows given in Table 2 for power-law background subtraction and signal integration.

NBED patterns were recorded with a self-written DigitalMicrograph plugin controlling the beam position and reading out an UltraScan $1000 \times P$ camera binned to $256 \times 256$ pixels.

In order to extract the lattice parameters from resulting NBED patterns, their auto-correlation is calculated in a first step. Subsequently, a $2 \mathrm{D}$ reciprocal lattice is fitted up to the second order to the positions of the local maxima at

$\mathbf{k}(i, j)=\left(k_{x}(i, j), k_{y}(i, j)\right),-2 \leq i, j \leq 2$

closest to the multiple orders of an initial guess $\left(i b_{10}+j \mathbf{b}_{20}\right)$ by solving the linear optimization problem:

$\min _{\mathbf{b}_{1}, \mathbf{b}_{2}} \sum_{i, j} \sum(i, j)^{2}\left(\mathbf{k}(i, j)-i \mathbf{b}_{1}-j \mathbf{b}_{2}\right)^{2}$

Here, $I(i, j)$ corresponds to the intensity of the local maximum at $\mathbf{k}(i, j)$ and serves as a weighting factor. Please note that this strategy of lattice parameter extraction is inspired by and thus very similar to those of previous reports. ${ }^{[42,55]}$ Throughout this paper, the manually estimated STO(110) and $\mathrm{STO}(001)$ reflections of the STO substrate at room temperature were used as initial guesses $\mathbf{b}_{10}$ and $\mathbf{b}_{20}$. In the orthorhombic Pbnm unit cell of PCMO, these reflections translate approximately to PCMO(100)/ $\mathrm{PCMO}(010)$ and $\mathrm{PCMO}(002)$. In order to obtain quantitative reciprocal lattice parameters, the electron-optical system was calibrated by assuming an unstrained substrate $\approx 50-100 \mathrm{~nm}$ below the interface at room temperature, as the Young modulus at room temperature is about three times higher than in PCMO $(x=0.5)^{[56,57]}$ and similar behavior is expected for $x=0.1$. The moduli of real space lattice parameters were calculated by taking the inverse of the moduli of the reciprocal lattice parameters. The standard deviation of the calibrated lattice parameter in the substrate reference area amounts to $3 \mathrm{pm}$ and serves as an estimation of potential systematic errors of the quantitative values.

XRD was performed in a Bruker D8 diffractometer with a monochromated $\mathrm{Cu} K \alpha \mathrm{l}$ source.

\section{Acknowledgements}

The project was funded by the Deutsche Forschungsgemeinschaft (DFG, German Research Foundation)-217133147/SFB 1073, projects B02, Z02. The use of equipment in the "Collaborative Laboratory and User Facility for Electron Microscopy" (CLUE, Göttingen) is gratefully acknowledged. Furthermore, the authors acknowledge the open acces software VESTA, ${ }^{[58]}$ which was used for grpahical illustration in Figure 1.

Open access funding enabled and organized by Projekt DEAL.

\section{Conflict of Interest}

The authors declare no conflict of interest.

\section{Author Contributions}

The design of experiments and their interpretation was the result of fruitful discussions including all authors. T.M. extracted the TEM lamellas from a thin film sample grown by B.K. and conducted the TEM experiments together with V.R. and B.K. The data was analyzed by T.M. under revision of M.S. The manuscript was written by T.M. under revision of M.S. and C.J.. All authors read and agreed on the written paper.

\section{Data Availability Statement}

The data presented in this work can be found under: https://doi. org/10.25625/STJOWN.

\section{Keywords}

environmental TEM, in situ heating, nano-beam electron diffraction, oxygen vacancy, phase transitions, thin film perovskites

Received: June 18, 2021

Published online:

[1] V. K. Sangwan, M. C. Hersam, Nat. Nanotechnol. 2020, 7, 517.

[2] J. W. Park, B. H. Kang, H. J. Kim, Adv. Funct. Mater. 2020, 30, 1904632.

[3] X. Su, Q. Wu, J. Li, X. Xiao, A. Lott, W. Lu, B. W. Sheldon, J. Wu, Adv. Energy Mater. 2014, 4, 1300882.

[4] W. Hsu, C. M. Sutter-Fella, M. Hettick, L. Cheng, S. Chan, Y. Chen, Y. Zeng, M. Zheng, H.-P. Wang, C.-C. Chiang, et al., Sci. Rep. 2015, 5, 16028.

[5] A. Guerrero, S. Chambon, L. Hirsch, G. Garcia-Belmonte, Adv. Funct. Mater. 2014, 24, 6234.

[6] Q. Yang, Y. Liu, C. Pan, J. Chen, X. Wen, Z. L. Wang, Nano Lett. 2013, 13, 607.

[7] O. Lupan, T. Pauporté, B. Viana, Adv. Mater. 2010, 22, 3298.

[8] Y. Wang, H. Arandiyan, H. A. Tahini, J. Scott, X. Tan, H. Dai, J. D. Gale, A. L. Rohl, S. C. Smith, R. Amal, Nat. Commun. 2017, 8, 1.

[9] J. Scholz, M. Risch, K. A. Stoerzinger, G. Wartner, Y. Shao-Horn, C. Jooss, J. Phys. Chem. C 2016, 120, 27746.

[10] L. M. Loong, X. Qiu, Z. P. Neo, P. Deorani, Y. Wu, C. S. Bhatia, M. Saeys, H. Yang, Sci. Rep. 2014, 4, 6505.

[11] S.-C. Oh, S.-Y. Park, A. Manchon, M. Chshiev, J.-H. Han, H.-W. Lee, J.-E. Lee, K.-T. Nam, Y. Jo, Y.-C. Kong, B. Dieny, K.-J. Lee, Nat. Phys. 2009, 5, 898.

[12] S. Yuasa, T. Nagahama, A. Fukushima, Y. Suzuki, K. Ando, Nat. Mater. 2004, 3, 868

[13] L. Xue, S. V. Savilov, V. V. Lunin, H. Xia, Adv. Funct. Mater. 2018, 28, 1705836.

[14] Y. S. Jung, P. Lu, A. S. Cavanagh, C. Ban, G.-H. Kim, S.-H. Lee, S. M. George, S. J. Harris, A. C. Dillon, Adv. Energy Mater. 2013, 3, 213. 
[15] S. Luo, K. Wang, J. Wang, K. Jiang, Q. Li, S. Fan, Adv. Mater. 2012, 24, 2294.

[16] Q. Wan, M. T. Sharbati, J. R. Erickson, Y. Du, F. Xiong, Adv. Mater. Technol. 2019, 4, 1900037.

[17] D. S. Jeong, C. S. Hwang, Adv. Mater. 2018, 30, 1704729.

[18] S. Park, M. Chu, J. Kim, J. Noh, M. Jeon, B. H. Lee, H. Hwang, B. Lee, B.-G. Lee, Sci. Rep. 2015, 5, 10123.

[19] J. Zhang, Z. Zhong, X. Guan, X. Shen, J. Zhang, F. Han, H. Zhang, H. Zhang, X. Yan, Q. Zhang, L. Gu, F. Hu, R. Yu, B. Shen, J. Sun, Nat. Commun. 2018, 9, 1923.

[20] H. Jeen, W. S. Choi, J. W. Freeland, H. Ohta, C. U. Jung, H. N. Lee, Adv. Mater. 2013, 25, 3651.

[21] M. Janousch, G. I. Meijer, U. Staub, B. Delley, S. F. Karg, B. P. Andreasson, Adv. Mater. 2007, 19, 2232.

[22] D. Raiser, S. Mildner, B. Ifland, M. Sotoudeh, P. Blöchl, S. Techert, C. Jooss, Adv. Energy Mater. 2017, 7, 1602174.

[23] B. Ifland, J. Hoffmann, B. Kressdorf, V. Roddatis, M. Seibt, C. Jooss, New J. Phys. 2017, 19, 063046.

[24] B. Ifland, P. Peretzki, B. Kressdorf, P. Saring, A. Kelling, M. Seibt, C. Jooss, Beilstein J. Nanotechnol. 2015, 6, 1467.

[25] G. Lole, V. Roddatis, U. Ross, M. Risch, T. Meyer, L. Rump, J. Geppert, G. Wartner, P. Blöchl, C. Jooss, Commun. Mater. 2020, $1,68$.

[26] D. Mierwaldt, V. Roddatis, M. Risch, J. Scholz, J. Geppert, M. E. Abrishami, C. Jooss, Adv. Sustainable Syst. 2017, 1, 1700109.

[27] S. Mildner, M. Beleggia, D. Mierwaldt, T. W. Hansen, J. B. Wagner, S. Yazdi, T. Kasama, J. Ciston, Y. Zhu, C. Jooss, J. Phys. Chem. C 2015, 119, 5301

[28] S. Raabe, D. Mierwaldt, J. Ciston, M. Uijttewaal, H. Stein, J. Hoffmann, Y. Zhu, P. Blöchl, C. Jooss, Adv. Funct. Mater. 2012, 22, 3378.

[29] Y. Tokura, N. Nagaosa, Science 2000, 288, 462.

[30] P. Beaud, A. Caviezel, S. Mariager, L. Rettig, G. Ingold, C. Dornes, S. Huang, J. Johnson, M. Radovic, T. Huber, et al., Nat. Mater. 2014, 13, 923.

[31] Z. Sheng, M. Nakamura, F. Kagawa, M. Kawasaki, Y. Tokura, Nat. Commun. 2012, 3, 1 .

[32] R. von Helmolt, J. Wecker, B. Holzapfel, L. Schultz, K. Samwer, Phys. Rev. Lett. 1993, 71, 2331.

[33] E. Pollert, S. Krupička, E. Kuzmičová, J. Phys. Chem. Solids 1982, 43, 1137.

[34] Z. Jirak, S. Krupička, Z. Šimša, M. Dlouha, S. Vratislav, Journal of Magnetism and Magnetic Materials 1985, 53, 153.

[35] Z. Jirák, J. Hejtmánek, K. Knıžek, R. Sonntag, J. Solid State Chem. 1997, 132, 98.

[36] D. Sánchez, J. Alonso, M. Martínez-Lope, J. Chem. Soc., Dalton Trans. 2002, 23, 4422.

[37] B. Kressdorf, T. Meyer, M. ten Brink, C. Seick, S. Melles, N. Ottinger, T. Titze, H. Meer, A. Weisser, J. Hoffmann, S. Mathias, H. Ulrichs,
D. Steil, M. Seibt, P. E. Blöchl, C. Jooss, Phys. Rev. B 2021, 103, 235122.

[38] J. Hwang, Z. Feng, N. Charles, X. R. Wang, D. Lee, K. A. Stoerzinger, S. Muy, R. R. Rao, D. Lee, R. Jacobs, et al., Mater. Today 2019, 31, 100.

[39] J. Yang, S. Hu, Y. Fang, S. Hoang, L. Li, W. Yang, Z. Liang, J. Wu, J. Hu, W. Xiao, et al., ACS Catal. 2019, 9, 9751.

[40] E. Enriquez, A. Chen, Z. Harrell, P. Dowden, N. Koskelo, J. Roback, M. Janoschek, C. Chen, Q. Jia, Sci. Rep. 2017, 7, 46184.

[41] S. Middey, J. Chakhalian, P. Mahadevan, J. Freeland, A. J. Millis, D. Sarma, Annu. Rev. Mater. Res. 2016, 46, 305.

[42] A. Béché, J. L. Rouvière, L. Clément, J. Hartmann, Appl. Phys. Lett. 2009, 95, 123114

[43] A. Glazer, Acta Crystallogr. Sect. B: Structural.Crystallogr. Crystal Chem. 1972, 28, 3384.

[44] F. Aguirre-Tostado, A. Herrera-Gomez, J. Woicik, R. Droopad, Z. Yu, D. Schlom, P. Zschack, E. Karapetrova, P. Pianetta, C. Hellberg, Phys. Rev. B 2004, 70, 201403.

[45] B. Ifland, J. Hoffmann, T. Kramer, M. Scherff, S. Mildner, C. Jooss, J. Nanomater. 2015, 2015.

[46] J. Hoffmann, P. Moschkau, S. Mildner, J. Norpoth, C. Jooss, L. Wu, Y. Zhu, Mater. Res. Express 2014, 1, 046403.

[47] X. Li, C. Lu, J. Dai, S. Dong, Y. Chen, N. Hu, G. Wu, M. Liu, Z. Yan, J.-M. Liu, Sci. Rep. 2014, 4, 1.

[48] H. Nakano, M. Kobayashi, N. Kamegashira, J. Am. Ceram. Soc. 2007, 90, 1276

[49] D. de Ligny, P. Richet, Phys. Rev. B 1996, 53, 3013.

[50] U. Aschauer, R. Pfenninger, S. M. Selbach, T. Grande, N. A. Spaldin, Phys. Rev. B 2013, 88, 054111

[51] D. I. Woodward, I. M. Reaney, Acta Crystallogr. Sect. B: Struct. Sci. 2005, 61, 387

[52] G. Saucke, J. Norpoth, C. Jooss, D. Su, Y. Zhu, Phys. Rev. B 2012, 85, 165315.

[53] T. Meyer, T. Westphal, B. Kressdorf, U. Ross, C. Jooss, M. Seibt, Ultramicroscopy 2021, 113320.

[54] F. de la Peña, E. Prestat, V. T. Fauske, P. Burdet, P. Jokubauskas, M. Nord, T. Ostasevicius, K. E. MacArthur, M. Sarahan, D. N. Johnstone, J. Taillon, J. Lähnemann, V. Migunov, A. Eljarrat, J. Caron, T. Aarholt, S. Mazzucco, M. Walls, T. Slater, F. Winkler pquinn dls, B. Martineau, G. Donval, R. McLeod, E. R. Hoglund, I. Alxneit, D. Lundeby, T. Henninen, L. F. Zagonel, A. Garmannslund, hyperspy/hyperspy: HyperSpy v1.5.2 2019, https://doi.org/10.5281/ zenodo.3396791.

[55] F. H. Baumann, Appl. Phys. Lett. 2014, 104, 262102.

[56] M. A. Carpenter, C. J. Howard, R. E. McKnight, A. Migliori, J. B. Betts, V. R. Fanelli, Phys. Rev. B 2010, 82, 134123.

[57] A. Hachemi, H. Hachemi, A. Ferhat-Hamida, L. Louail, Phys. Scr. $2010,82,025602$

[58] K. Momma, F. Izumi, J. Appl. Crystallogr. 2011, 44, 1272. 


\subsection{Low Temperature Investigations for $x=0.1$}

Similarly to the experiment conducted on PCMO $(x=0.34)$ and presented above in the context of charge ordering, SAED of PCMO $(x=0.1)$ was performed at low temperatures in order to check for fingerprints of orbital ordering [28]. As conventionally prepared samples reproducibly cracked during thinning in the PIPS - possibly due to the complex strain-stress state discussed in the previous subsection - the combined mechanical grinding and FIB lift-out approach presented in subsection 4.1.1 was used to prepare a planview oriented TEM lamella of a $400 \mathrm{~nm}$ thick film. (see Tab. A.1 for details of the macroscopic preparation). Fig. 6.5 (a) shows a TEM overview of a region in which the substrate has been removed entirely, i.e. with a thickness well below $400 \mathrm{~nm}$ as can be confirmed by EELS. The dashed yellow lines indicate domain boundaries predominantly following the (110) resp. (11̄0) planes with alternating in-plane orientation. Interestingly, the domains tend to be elongated in one direction as well as closely spaced in the other and areas with interchanging long resp. short edges exist. Due to a stronger superposition with bending contrasts, the horizontally aligned long edges in the thinner left part are less clearly visible, but can be confirmed by the defocused image with higher magnification given in Fig. A.12. The five marked positions in Fig. 6.5 (a) indicate regions investigated with SAED at $300 \mathrm{~K}$ (red) reps. $100 \mathrm{~K}$ (blue) and exemplary DPs are shown in (b) and (c). Consistent with the sketch presented in Fig. 2.8, no superstructure as a consequence of orbital ordering is observed at low temperatures, which can also be confirmed in thicker sample regions. However, as discussed in chapter 2 and 5 , an increase in the structural mode $q_{3}$ is expected due to the collaborative JT effect leading to an enhanced anisotropy of the in-plane lattice parameters, i.e. an increase of the ratio $b / a$. In order to check if such a structural fingerprint can be resolved with electron diffraction, the reflections with clearly distinguishable double peaks resulting from the inclusion of scattering volumes from both domain orientations and marked with green circles in Fig. 6.5 (b) were fitted for each orientation to a two-dimensional lattice using Eq. (4.1). Here, no auto- or cross-correlation was performed but the peak maxima in the direct DP was used. Furthermore, the weighting factor was chosen uniformly to be $24^{-1} \Delta k^{-2}$, where $\Delta k=0.013 \mathrm{~nm}^{-1}$ is the pixel spacing of the DP, yielding a reduced $\chi^{2}$ form allowing for an error estimation of the fit parameters. Given the in-plane lattice parameters for each domain orientation, the following quantities can be calculated: Firstly, the angle between the lattice planes along the elongated domain walls, i.e. between the vertically aligned (110) resp. (11̄0) reflections fol- 


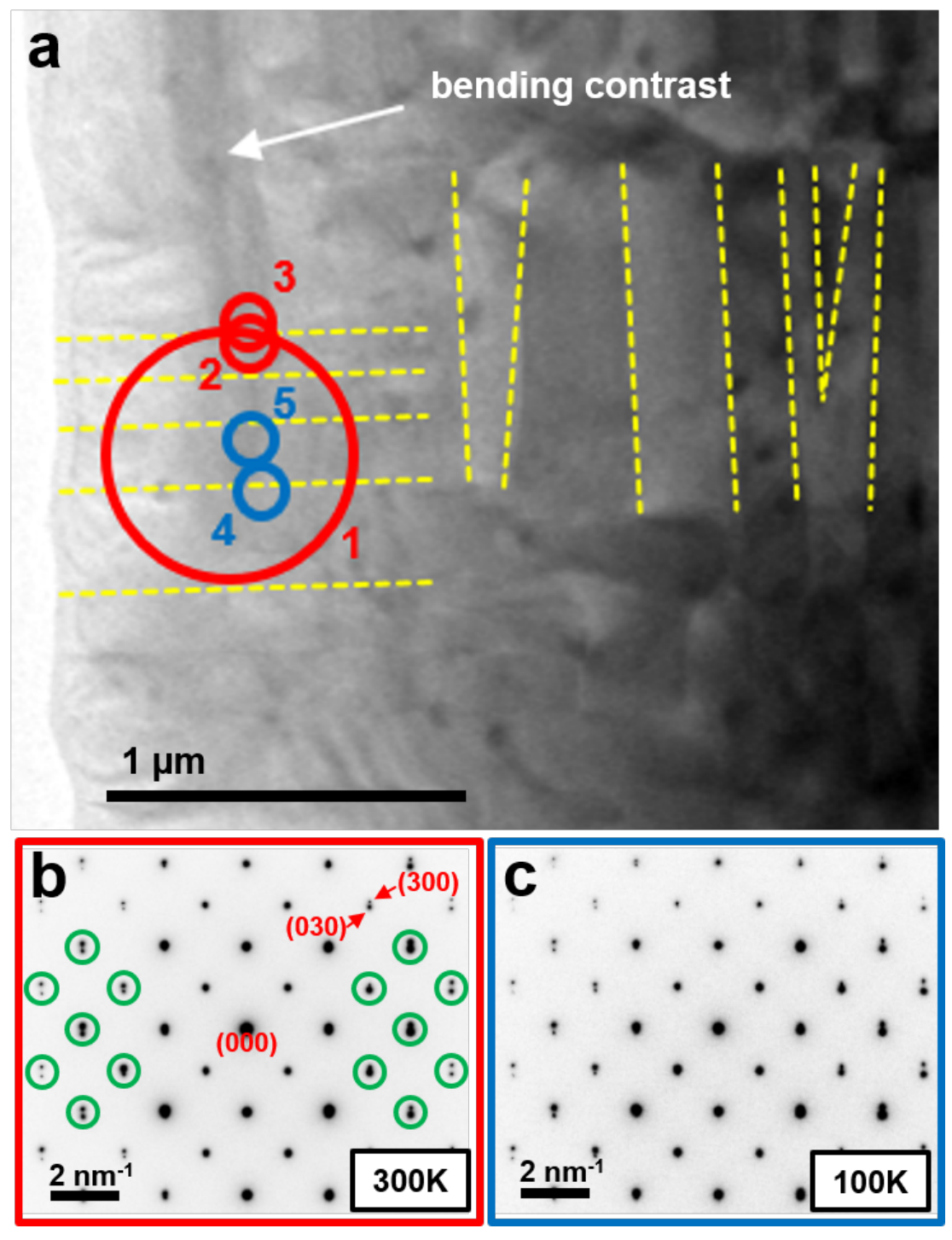

Figure 6.5: (a) TEM overview of a freestanding PCMO $(x=0.1)$ lamella with alternating domain structure. The dashed yellow lines indicate domain boundaries and the red resp. blue circles correspond to regions investigated by SAED. Exemplary DPs at $300 \mathrm{~K}$ and $100 \mathrm{~K}$ are shown in (b) and (c). The double peaks marked with green circles were used to extract the lattice parameters of the sublattices corresponding to the differently oriented domains. 

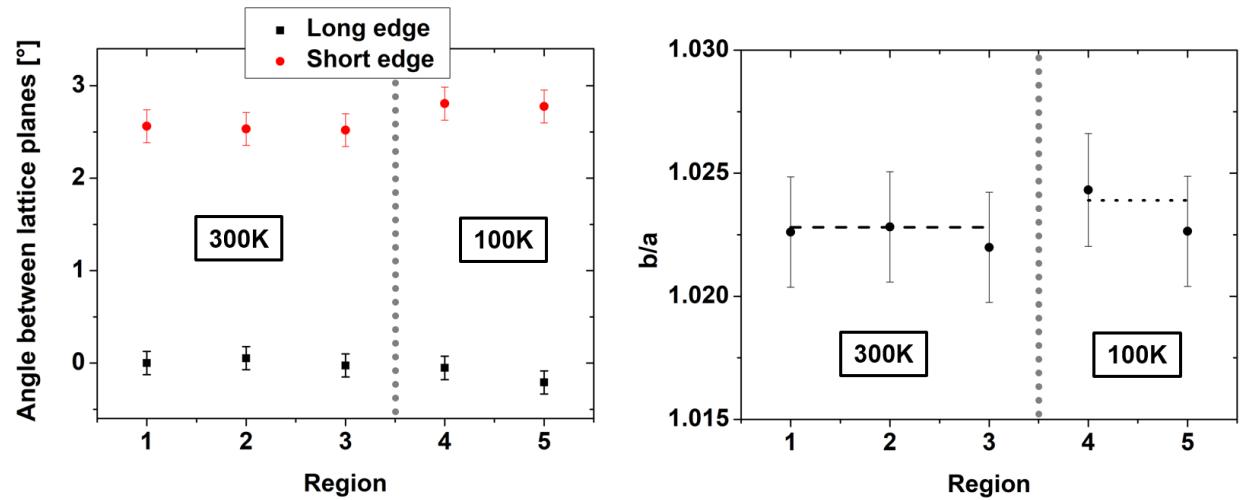

Figure 6.6: Parameters extracted from the SAED patterns taken in the five regions marked in Fig. 6.5 (a): (Left) Angle between the lattice planes in adjacent domains along the long resp. short edges of the domains structure, i.e. between the vertical reps. horizontal diffraction spots of the two sublattices in Fig. 6.5 (b) and (c). (Right) Ratio of the in-plane lattice parameters $b / a$ averaged over both sublattices as well as the corresponding bulk values from [161] marked by the dashed $(283 \mathrm{~K})$ resp. dotted $(83 \mathrm{~K})$ line.

lowing the index convention shown in Fig. 6.5 (b). Secondly, the angle between the horizontally aligned (110) resp. (110) reflections. Thirdly, the ratio $b / a$ averaged over both domain orientations. The resulting values for the five regions marked in Fig. 6.5 (a) are presented in Fig. 6.6 showing that the (110) and (110) lattice planes at the long edges tend to be parallel to each other while the (110) and (110) planes are inclined by more than $2.5^{\circ}$. Qualitatively, this behaviour can already be inferred from the absence resp. emergence of double peaks in the vertical resp. horizontal direction in Fig. 6.5 (b) and (c). However, despite being poorly statistically sampled, the comparison between data points being collected at $300 \mathrm{~K}$ and $100 \mathrm{~K}$ suggests an increase in the inclination angle at low temperatures being consistent with the expected enhanced anisotropy of the in-plane lattice parameters [161]. This tendency is also visible in the comparatively noisy direct plot of $b / a$ with the bulk ratios from the latter reference marked by the dashed $(283 \mathrm{~K})$ resp. dotted $(83 \mathrm{~K})$ line. Obviously, as the main intention of this experiment was to confirm that no detectable superstructure emerges at low temperatures, the recorded sample set is insufficient to draw strong conclusions about orbital ordering. In particular, the comparison of solely two different temperatures impedes the disentanglement of thermal 
expansion as well as effects caused by the alternating domain structure from possible fingerprints of orbital ordering. Nevertheless, the results show that SAED in combination with the used planview sample preparation approach, i.e. with substrate removal, is suitable to study fingerprints of orbital ordering as the in-plane parameters can be determined individually and the sensitivity seems to be high enough to extract significant trends at reasonably low sample sizes. In fact, since cooling experiments are accompanied by comparatively strong specimen drift, the latter is an important experimental requirement. Noteworthy, the inclination angle of the lattice planes along the short domain walls can be extracted rather precisely from the double peaks and is a promising candidate to observe the in-plane anisotropy in future experiments. Furthermore, the collection of SAED patterns with solely the systematic row along $a$ resp. $b$ excited should be included as, according to Fig. 2.8, the orbital ordering is expected to (probably weakly) lift the extinction of the (100) resp. (010) reflection since the central Mn site of the Pbnm unit cell is no longer equivalent to the corner site. However, in the herein chosen zone axis [001], double diffraction including allowed (210) and $(\overline{2} 00)$ resp. (120) and $(0 \overline{2} 0)$ reflections [164] lead already to high intensities at (100) resp. (010) impeding such an observation. 


\section{Chapter 7}

\section{Scanning Transmission Electron Beam Induced Current}

In this chapter, experimental advances of STEM-EBIC as well as those related to analytical and numerical modelling of resulting signals shall be presented. Firstly, in order to assess how results obtained in TEM lamellas can be linked to bulk devices, i.e. to quantify bulk-like contributions to the $I-V$ characteristic of the specimen as well as the MEMS carrier chip, the one diode model will be applied and underlying parallel as well as serial resistances will be quantified. Secondly, a qualitative proof of the superior spatial resolution compared with SEM-EBIC will be presented in the context of enhanced excess charge carrier recombination in the nanoscale vicinity of misfit dislocations at PCMO-STNO interfaces. Furthermore, it will be shown that a reliable extraction of the bulk diffusion length and surface properties of STNO is feasible as well as that in-situ biasing can be used to manipulate PCMO-STNO junctions an the nanoscale. In addition, the applicability to classical semiconductors, i.e. a textured silicon solar cell, will be proven enabling the mapping of a boron isoconcentration line in a non-planar geometry by correlating the experiment with $\mathrm{FE}$ and boron diffusion simulations. Lastly, the existence of atomically modulating EBIC signals in PCMO, STNO, and Si will be presented and discussed in the context of possible energy dissipation channels after primary excitation of the electron beam as well as their spatial evolution with respect to the position within the space charge region. 


\subsection{Applying the One Diode Model to Electron Transparent Lamellas}

In order to extract the bulk-like contributions to the $I$ - $V$ curve of electron transparent lamella mounted on a MEMS chip, the one diode model presented in subsection 2.2.3 is be employed in a two-step procedure. Firstly, Eq. (2.19) is fitted to data obtained on the underlying macroscopic sample yielding the corresponding saturation current $I_{\mathrm{S}}$ as well as ideality factor $n$. Secondly, the one diode model - including a fraction $r$ of the macroscopic device with identical ideality factor, as well as additional serial and parallel resistances $R_{\mathrm{S}}$ and $R_{\mathrm{p}}$ caused by the preparation - is fitted by solving the following optimisation problem:

$$
\min _{r, R_{\mathrm{s}}, R_{\mathrm{p}}} \frac{1}{(N-3) \sigma_{I}^{2}} \sum_{i=0}^{N}\left(I\left(U_{i}, r, R_{\mathrm{s}}, R_{\mathrm{p}}\right)-I_{i}\right)^{2} .
$$

Here, $I_{i}$ is the resulting current at an external voltage $U_{i}$ applied to the chip contacts and $N$ the amount of collected data points. Furthermore, $\sigma_{I}=0.01 \mathrm{nA}$ denotes the uncertainty of the current measurement, i.e. Eq. (7.1) yields the reduced $\chi^{2}$, and $I\left(U_{i}, r, R_{\mathrm{s}}, R_{\mathrm{p}}\right)$ is implicitly defined by

$$
0=I\left(1+\frac{R_{\mathrm{s}}}{R_{\mathrm{p}}}\right)-\frac{U_{i}}{R_{\mathrm{p}}}-r I_{\mathrm{S}}\left(\exp \left(\frac{e\left(U_{i}-R_{\mathrm{s}} I\right)}{n k_{\mathrm{B}} T}\right)-1\right) .
$$

The $I-V$ curves of the wedge-shaped PCMO-STNO lamella presented at the bottom of Fig. 4.2 (black) as well as the corresponding macroscopic sample (blue) are presented in Fig. 7.1. Clearly, large current contributions due to a finite parallel resistance are visible in the sample prepared for STEM-EBIC purposes. However, no claims about the origin of related leakage currents can be made in the first place, i.e. it is not clear whether short circuits emerge due to the FIB treated surfaces or contaminations on the MEMS chip e.g. due to Pt deposition. Solving Eq. (7.1) for the presented dataset yields optimal values $r=1.66(13) \times 10^{-4}, R_{\mathrm{s}}=44(2) \mathrm{M} \Omega$, and $R_{\mathrm{p}}=436(14) \mathrm{M} \Omega$. In the framework presented previously in Fig. 2.11, these values translate to a ratio of 0.87 between experimentally measured and actual short circuit currents and subsequently shown EBIC data obtained on the presented lamella will be corrected by dividing through the latter unless stated otherwise. In order to demonstrate how accurately the experimentally measured $I-V$ curve can be described by the applied one

diode model, the data is corrected for effects caused by $R_{\mathrm{S}}$ and $R_{\mathrm{p}}$ following 


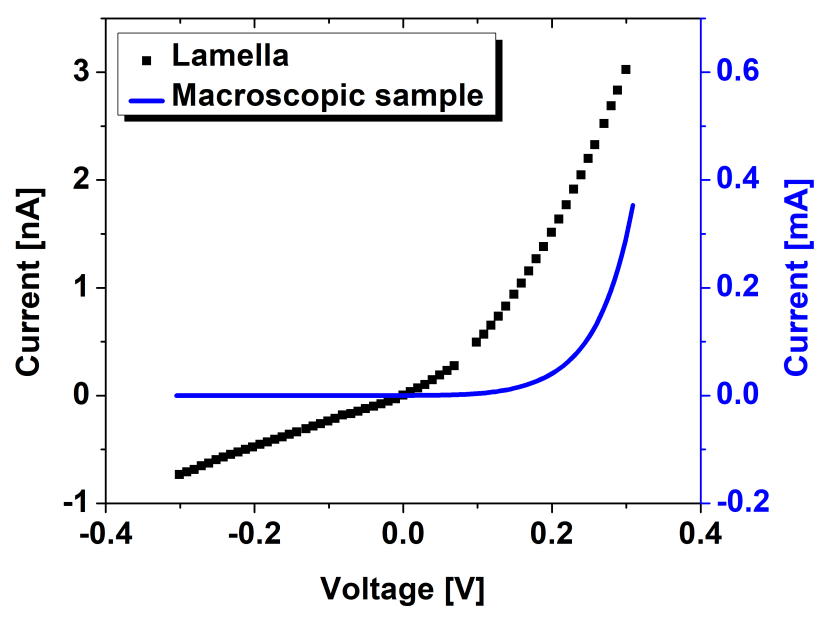

Figure 7.1: $I-V$ curves of the wedge-shaped PCMO-STNO lamella shown at the bottom of Fig. 4.2 (black) as well as the corresponding macroscopic sample (blue). See Tab. A.1 for details of the macroscopic sample.

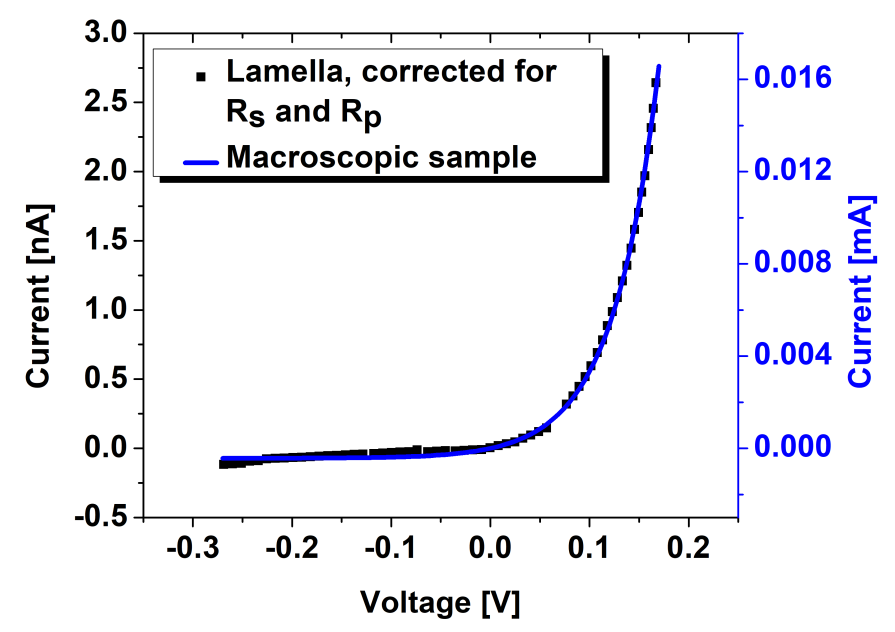

Figure 7.2: $I-V$ curves of the wedge-shaped PCMO-STNO lamella shown at the bottom of Fig. 4.2 after correction for the effects of $R_{\mathrm{S}}$ and $R_{\mathrm{p}}$ as obtained by the fit to the one diode model (black) as well as the corresponding macroscopic sample (blue). The scales were adjusted by the ratio $r$. 


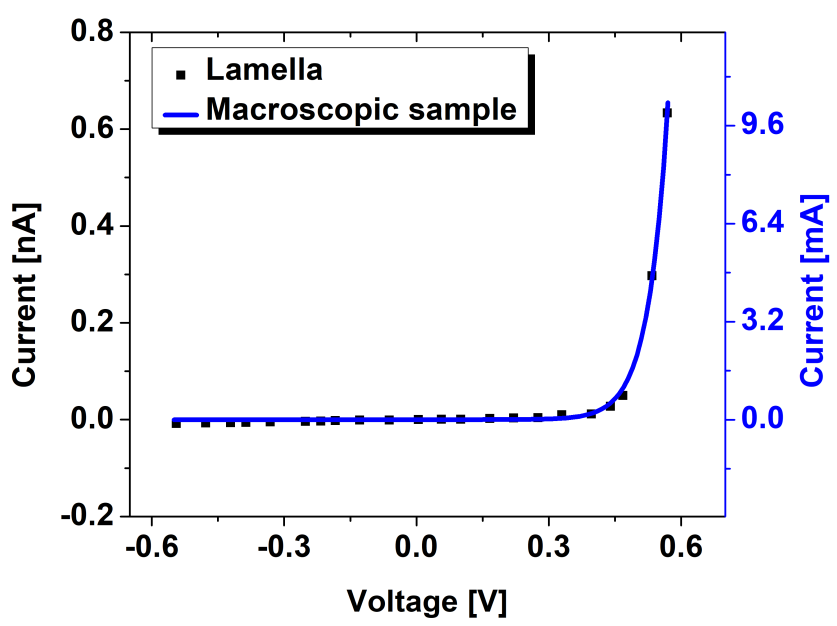

Figure 7.3: $I-V$ curves of a wedge-shaped (RP-PCMO)-STNO lamella prepared analogously to the one shown in Fig. 7.1 (black) as well as the corresponding macroscopic sample (blue). See Tab. A.1 for details of the macroscopic sample.

$U_{i}^{\text {corr }}=\left(U_{i}-R_{\mathrm{s}} I_{i}\right)$ resp. $I_{i}^{\text {corr }}=\left(I_{i}-U_{i}^{\text {corr }} / R_{\mathrm{p}}\right)$ and plotted in Fig. 7.2 showing a remarkable match with the macroscopic behaviour. The scales of the respective current axes are adjusted by the extracted value of $r$. Applying the same analysis to the uniformly thinned lamella with contacts on opposite sides shown at the top of Fig. 4.2 yields $r=6.8(3) \times 10^{-6}, R_{\mathrm{S}}=8.4(7) \mathrm{M} \Omega$, as well as $R_{\mathrm{p}}=469(14) \mathrm{M} \Omega$ which translates to a correction factor of 0.98 . The reduced value of $r$ compared with the wedge-shaped lamella reflects the smaller active area in the homogeneously thin and narrower sample while the deviations of the resistances are within the fluctuations of analogously fabricated TEM foils. In all preparations, the chip was cut in between the platinum contacts along several tens of micrometers to reduce short circuits due to large-scale contaminations and the same ion acceleration voltages and currents were used during thinning. However, the $I-V$ characteristic of a wedge-shaped (RP-PCMO)-STNO lamella without any correction presented in Fig. 7.3 shows that in extreme cases the effect of serial and parallel resistances can be negligibly small. Noteworthy, the results presented in this section show that in principle, even if the latter is not the case, effects of finite values of $R_{\mathrm{s}}$ and $R_{\mathrm{p}}$ can be corrected for and the demonstrated procedure allows for a quantitative comparison. 


\subsection{STEM-EBIC of Misfit Dislocations at PCMO- STNO Interfaces}

Given the confined interaction of the electron beam with the sample, the following paper [165] on enhanced recombination in the nanoscale vicinity of misfit dislocations at PCMO-STNO interfaces was published in Journal of Physics: Conference Series (doi.org/10.1088/1742-6596/1190/1/012009) - the author's contributions are given below. All contents are reprinted in their original form with permission.

Besides the experimental proof that cross-sectional STEM-EBIC in the underlying materials is feasible at ultralow sample thicknesses of approximately $40 \mathrm{~nm}$, a qualitatively different behaviour of frequently occuring misfit dislocations depending on the location of the dislocation core was revealed. More specifically, dislocations with cores well-located in the lowerdoped STNO substrate show a much more pronounced EBIC reduction compared with those exhibiting cores directly at the interface. In either way, the dislocation is located in the expected domain of the SCR making the modelling of enhanced recombination processes extremely challenging since electric and strain fields caused by the junction, the crystal defect and potentially charged surfaces need to be accounted for simultaneously. Therefore, in the scope of the presented paper, solely qualitative conclusions are drawn and the role of enhanced recombination in field-immersed sample regions as well as due to the damaged surfaces will be discussed in the context of subsequently obtained results later on.

\section{Author contributions}

T.M. extracted the TEM lamella from the macroscopic sample grown by B.K. and conducted the TEM experiments with support by P.P. and V.R.; The DM resp. LabVIEW scripts for data acquisition where implemented by T.M. resp. J.L.; The manuscript was written by T.M. under revision of M.S and C.J.; All authors read and agreed on the written paper and declare no conflicts of interest. 


\title{
High-resolution Scanning Transmission EBIC Analysis of Misfit Dislocations at Perovskite pn-Heterojunctions
}

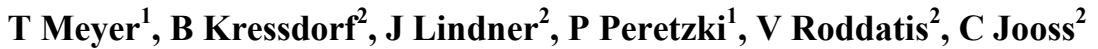 \\ and $\mathrm{M}$ Seibt ${ }^{1}$ \\ ${ }^{1} 4$ th Institute of Physics - Solids and Nanostructures, University of Goettingen, \\ Friedrich-Hund-Platz 1, 37077 Goettingen, Germany \\ ${ }^{2}$ Institute of Materials Physics, University of Goettingen, Friedrich-Hund-Platz 1, \\ 37077 Goettingen, Germany \\ tmeyer@uni-goettingen.de
}

\begin{abstract}
Fundamental losses of photovoltaic energy conversion are transmission of sub band gap photons and thermalisation which are the underlying physics of the Shockley-Queisser limit defining maximum conversion efficiency of single-junction solar cells. Strongly correlated materials such as perovskites are promising candidates to exceed this limit by exploiting (i) long wavelength light absorption and (ii) the existence of long-living intraband excitations indicating that harvesting hot excess carriers might be feasible in such systems. In this work, we study pn-heterojunctions produced from $\operatorname{Pr}_{1-x} \mathrm{Ca}_{x} \mathrm{MnO}_{3}$ on $\mathrm{SrTi}_{1-y} \mathrm{Nb}_{y} \mathrm{O}_{3}$ by means of microscopic techniques. Such systems exhibit relevant quantities such as space charge layer width, screening lengths and excess carrier diffusion lengths in the 1-10 nm range which makes the use of standard methods such as electron beam induced current a challenging task. We report scanning transmission electron beam induced current experiments of misfit dislocations at the heterojunction. The dislocation-induced reduction of the charge collection is studied with nanometer spatial resolution. Effects of surface recombination and the heterojunction electric field are discussed.
\end{abstract}

\section{Introduction}

Transition metal oxide perovskites, in particular manganites, became popular in the 1950s due to their extraordinary magnetic properties [1]. Ever since then the interest in this class of strongly correlated materials has grown continuously and various extraordinary phenomena such as high-temperature superconductivity [2], colossal magnetoresistance [3], and photovoltaic activity [4] have been discovered. One reason for the diversity of research projects related to perovskites is the high adjustability of different degrees of freedom: The ideal perovskite structure $\mathrm{ABC}_{3}$ is cubic with $\mathrm{A}$ occupying the primitive, $\mathrm{B}$ the body-centred, and $\mathrm{C}$, symbolising oxygen for all compounds considered in this work, the face-centred sites. In addition, tetragonal and even orthorhombic perovskites exist resulting from small lattice deformations. The latter are typically related to rotations and distortions of the octahedron spanned by species $\mathrm{C}$ and depend highly on the particular choice of constituents as well as doping of the respective lattice sites [5]. Thus, bonding lengths and angles of neighbouring atoms can be adjusted by the samples' stoichiometry resulting in different overlaps of orbitals and hence a tunable interplay of exchange interactions [6-8].

Using organic halide compounds, the efficiency of perovskite based solar cells has been increased beyond $20 \%$ during the last decade [9] offering a competitive solution to silicon solar cells. Still, 
achieving long-term stability and explaining the underlying physical transport processes remain open challenges. Despite much lower photovoltaic energy conversion efficiencies, transition metal oxide perovskites can be considered as a model system to accomplish the second task. In fact, it has been shown that $\operatorname{Pr}_{1-x} \mathrm{Ca}_{x} \mathrm{MnO}_{3}$ exhibit strong infrared absorption [10]. While a thorough review on manganites in general can be found in [11], this article focuses on the particular compound $\mathrm{Pr}_{0.66} \mathrm{Ca}_{0.34} \mathrm{MnO}_{3}$, subsequently referred to as PCMO, exhibiting a positive Seebeck coefficient and thus being considered as a p-type material [12]. Used as an absorbing material, PCMO shows a tremendously better photovoltaic performance in a charge-ordered state where the lattice distortions mentioned above become cooperative $[13,14]$. Most noticeably, the corresponding polaronic excitations are intraband transitions. Although they could potentially relax via phonon scattering, they seem to be stabilised by the cooperative configuration. Hence, the basic assumption of hot carrier thermalisation being faster than typical charge collection times resulting in the Shockley-Queisser limit [15] might be overcome in strongly correlated materials. Consequently, perovskites are a promising candidate to exceed this limitation. In this work, the considered PCMO films are grown on $\mathrm{SrTi}_{0.998} \mathrm{Nb}_{0.002} \mathrm{O}_{3}$ substrates, subsequently referred to as $\mathrm{STNO}$, an n-type material showing polaronic transport behaviour [16]. Besides the advantageous characteristics for photovoltaic applications mentioned above, due to typically high doping levels and the low mobility of polaronic charge carriers, important physical properties of the resulting PCMO-STNO heterojunction such as the space charge region width as well as the screening and excess carrier diffusion lengths are in the $1-10 \mathrm{~nm}$ range [17] demanding for high-resolution technique to investigate the fundamental electric transport phenomena.

Electron beam induced current (EBIC) is a widespread technique to map the electronic response of rectifying devices stimulated by a local excitation with energetic electrons and can be easily implemented in scanning electron microscopes (SEM) [18]. Despite the well-focussed incident beam, resolutions in the nanometer range cannot be achieved, which is due to multiple scattering of electrons in the material resulting in a pear-shaped spread of the energy deposited by the beam [19]. The generation volume increases with increasing acceleration voltage, also referred to as the Bethe limit [20]. Complementary approaches to decrease the latter have been made to decrease the samples' dimension along the beam propagation direction resulting in transmission of the impinging electrons before the beam spreads severely [21,22]. This idea is not only applicable to EBIC but also to cathodoluminescence (CL) measurements [23] and in contrast to non-transmitting methods the generation volume decreases with increasing acceleration voltage, also referred to as the Goldstein limit [24].

In this work, we present how scanning transmission EBIC (STEBIC) can be applied to PCMOSTNO heterojunctions in order to visualise enhanced recombination at misfit dislocations on the nanometer scale.

\section{Experiment}

A $100 \mathrm{~nm}$ thick $\operatorname{Pr}_{0.66} \mathrm{Ca}_{0.34} \mathrm{MnO}_{3}$ film has been deposited on a commercial $\mathrm{SrTi}_{0.998} \mathrm{Nb}_{0.002} \mathrm{O}_{3}$ substrate by reactive Xe-ion beam sputtering with an oxygen partial pressure of $1.4 \cdot 10^{-4}$ mbar at $700^{\circ}$ C. Subsequently, the heterojunction has been annealed for 20 hours at $900^{\circ} \mathrm{C}$ in air and equipped with a $200 \mathrm{~nm}$ thick Pt front contact layer via room temperature Xe-ion beam sputtering.

TEM lamellae have been prepared on DENSsolutions four contacts biasing chips suitable for the Lightning D7+ holder inside an FEI Nova 600 NanoLab Dual Beam FIB and investigated with a Philips CM200 UT (BF-TEM) and an FEI Titan80-300 (DF-STEM and EBIC) at $200 \mathrm{kV}$ and 80 $\mathrm{kV} / 300 \mathrm{kV}$, respectively. Figure 1 shows a DF-STEM image of a final TEM lamella after Ga-ion milling following the recipe summarised in table 1 and reactive plasma cleaning for $2 \mathrm{~min}$. Electron energy loss spectroscopy confirms a quite uniform lamella thickness of roughly $40 \mathrm{~nm}$.

In order to measure the EBIC signal, the DENSsolutions holder was connected to a Stanford Research Systems SR570 current preamplifier, which converts the input current into an output voltage. 
The latter was read out using a Keithley 2000 digital multimeter controlled by a homemade National Instruments LabVIEW script. Synchronously, the electron beam position inside the TEM was adjusted by a homemade Gatan DigitalMicrograph script communicating with the LabVIEW script via a shared folder.

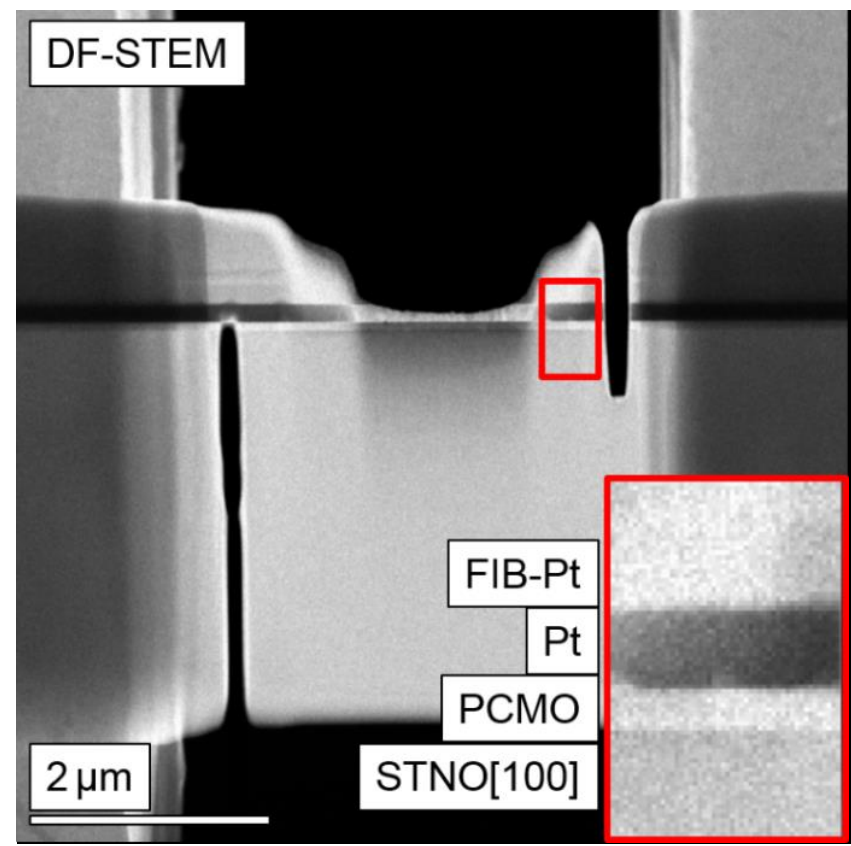

Figure 1. DF-STEM image of the FIB-prepared lamella: The enlargement of the small rectangular box marked illustrates the different layers of the sample. Vertical cuts have been made utilising lowdose (30 pA) Ga-ion milling with an acceleration voltage of $30 \mathrm{kV}$ in order to prevent short circuits across the pnjunction.

\begin{tabular}{cccc}
\hline $\begin{array}{c}\text { Acceleration } \\
\text { Voltage }(\mathrm{kV})\end{array}$ & $\begin{array}{c}\text { Ga-ion beam } \\
\text { current }(\mathrm{pA})\end{array}$ & $\begin{array}{c}\text { Tilt offset } \\
\left({ }^{\circ}\right)\end{array}$ & $\begin{array}{c}\text { Residual } \\
\text { thickness }(\mathrm{nm})\end{array}$ \\
\hline 30 & 300 & 1.2 & 800 \\
30 & 100 & 1.2 & 300 \\
5 & 29 & 2 & $<50$
\end{tabular}

Table 1. Milling recipe for the TEM lamellae preparation using Ga-ions. The residual thickness values are rough estimations obtained by the measurement tool of the FIB software and should be considered as a guide.

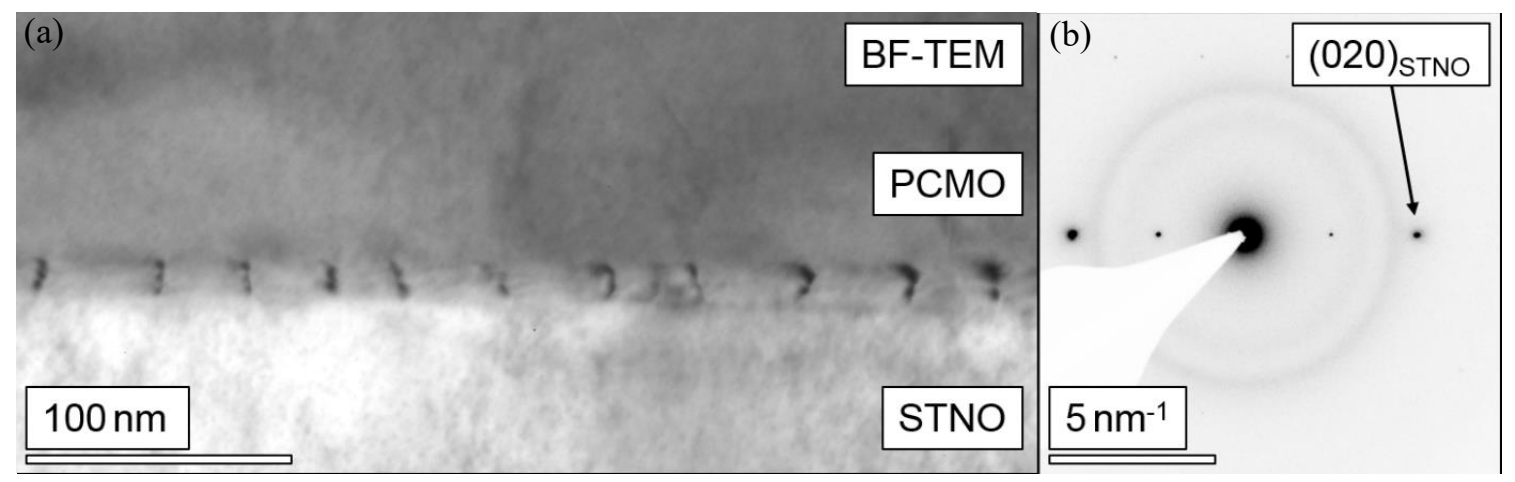

Figure 2. (a) BF-TEM image of the pn-junction revealing frequently occurring misfit dislocations with (b) corresponding diffraction pattern. Only the systematic row of $(010)_{\text {STNO }}$ reflections is excited, i.e. the interface is inclined by $20^{\circ}$ with respect to the incident electron beam leading to the vertical elongation of the dark contrasts. 


\section{Results and Discussion}

Given the high spatial resolution of STEBIC, local variations of the junction's short-circuit current can be detected. Typical causes for such variations are perturbations of the crystal symmetry resulting in enhanced recombination of excess charge carriers [25]. Due to the lattice misfit between film and substrate of approximately $f=-1.5 \%$ [26], defect formation to relax the corresponding stress are likely to occur. In fact, in PCMO/STNO thin film couples various relaxation possibilities like oxygen vacancy formation [27], twinning [28], and plastic relaxation due to dislocations [12] have been reported. Figure 2 shows that misfit dislocations occur frequently under the growth conditions described in the previous section. The mean distance between the observed dislocations of $33 \mathrm{~nm}$ is only $27 \%$ larger than the characteristic dislocation distance $d=a / f=26 \mathrm{~nm}$ for total stress relaxation via misfit dislocation formation (with $a$ being the mean in-plane lattice constant of PCMO [29]). Hence, a substantial fraction of the epitaxial stress is relaxed via this channel.

High-resolution DF-STEM images reveal that, despite having the same Burgers vector $[010]_{\text {STNO }}$, the dislocations have different characters: Figure 3(b) shows an interfacial dislocation (subsequently referred to as 'dislocation I') with an added half plane in the film. Since the unit cell of PCMO is larger than in STNO due to alternatingly tilted oxygen octahedra [30], the Burgers vector $[010]_{\text {STNO }}$ corresponds to a partial dislocation in the PCMO which is probably accompanied by a stacking fault in the tilt system. The related strain could induce chemical disorder, e.g. vacancies, leading to the dark vertical contrast emerging at the interface. On the contrary, the core of the buried dislocation in figure 4(b) (subsequently referred to as 'dislocation II') lies approximately five unit cells below the junction and the alternating tilting pattern of the PCMO film above seems to be undisturbed. The corresponding EBIC maps in figure 3(a) and figure 4(a) show that the induced recombination behaviour is significantly different.

Dislocation I leads to a small kink in the EBIC signal marked by the black circle but no medium range effect. A straight forward explanation would be to assume that dislocation I does not introduce deep states into the gap of PCMO. It should be kept in mind, however, that the screening length in PCMO [31] as well as the excess carrier diffusion length [14] are in the nanometer range indicating that the effect of deep states on the EBIC signal should be small. In contrast, dislocation II reduces the EBIC signal by roughly $30 \%$ in the vicinity of the core as shown by the averaged horizontal line

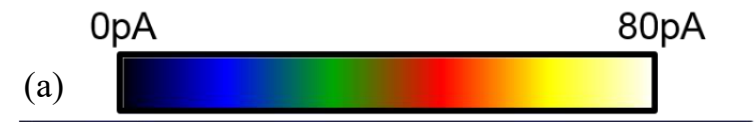

(b)
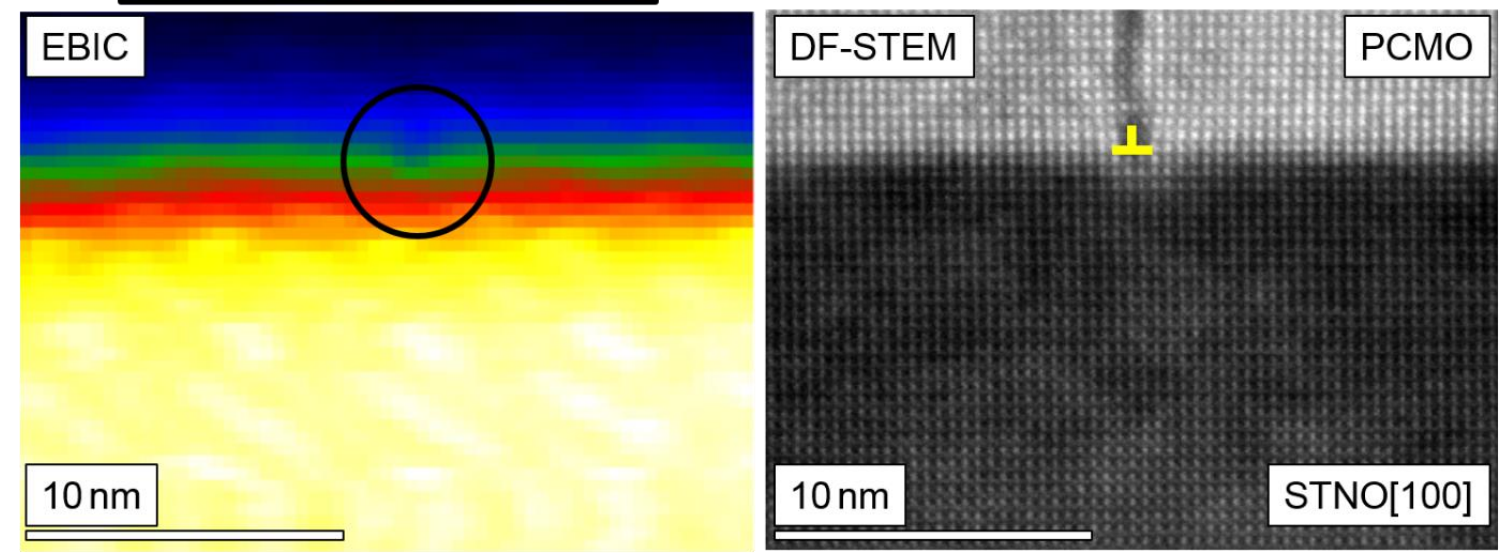

Figure 3. (a) EBIC map of an interfacial edge dislocation and (b) the corresponding DF-STEM micrograph. The dislocation is accompanied by a stacking fault in the PCMO layer due to its larger unit cell and has little effect on the EBIC signal. 


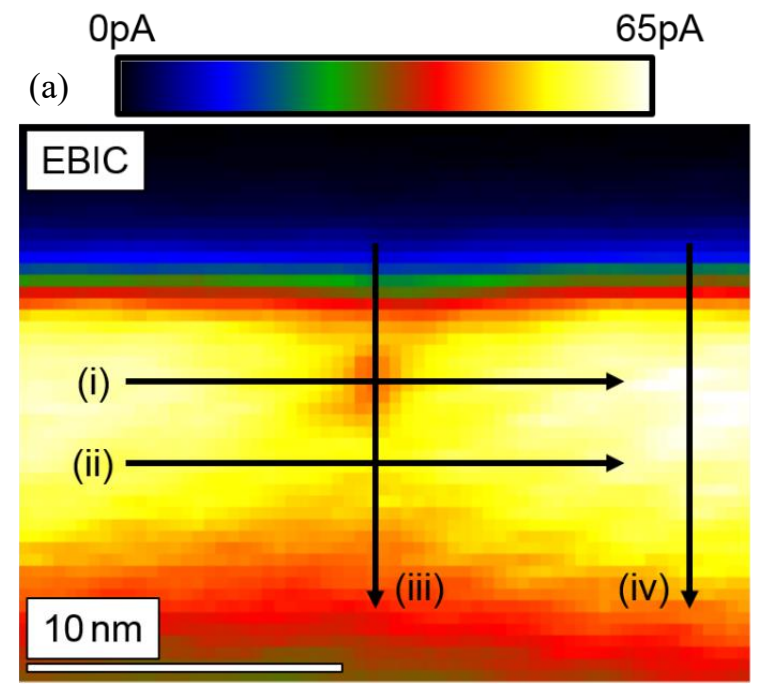

(b)

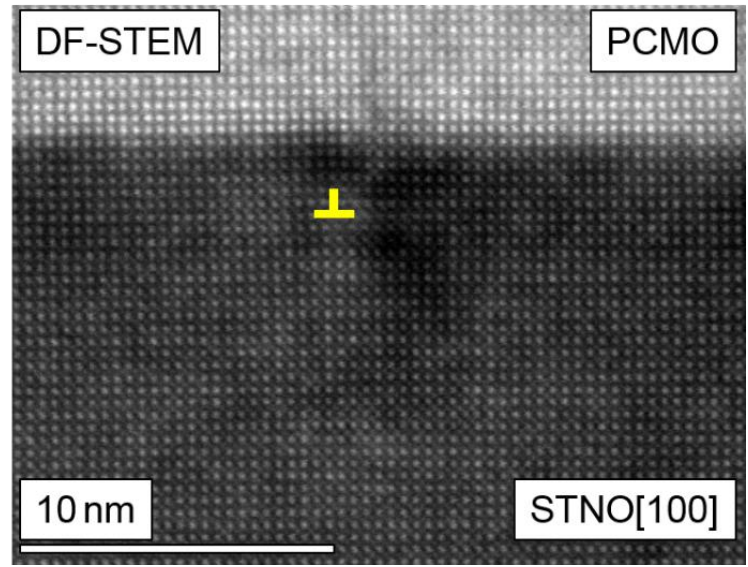

Figure 4. (a) EBIC map of a buried edge dislocation and (b) the corresponding DF-STEM micrograph. The dislocation reduces the EBIC signal significantly. The black arrows in (a) indicate spatial directions considered in figure 5 .
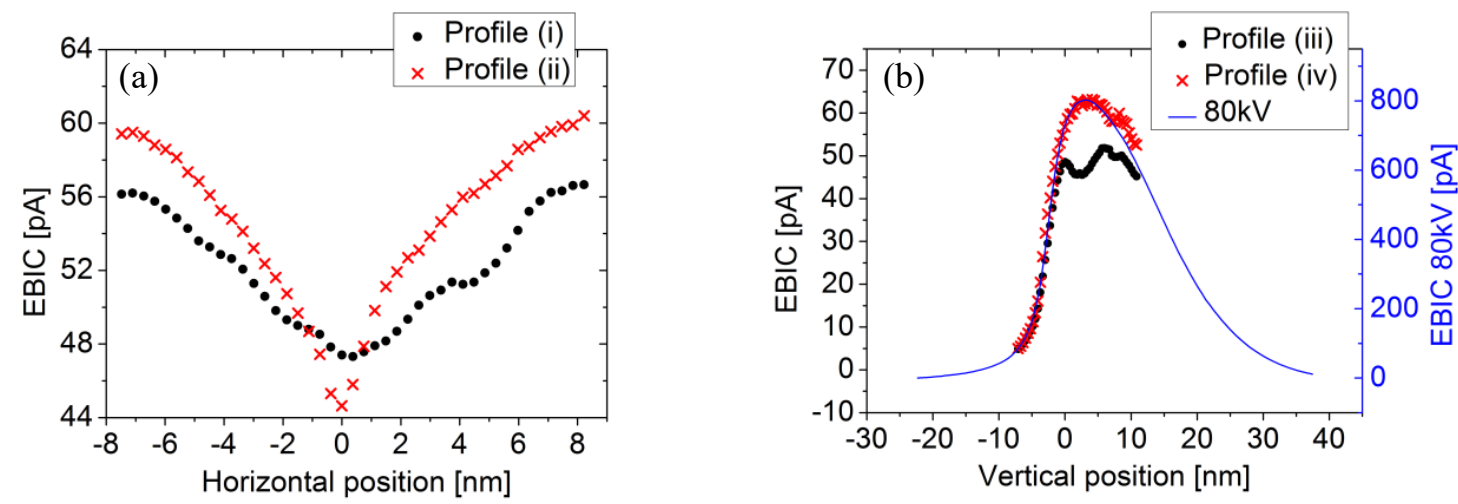

Figure 5. EBIC profile taken along (a) the horizontally aligned arrows in figure 4(a) (averaged over five lines) and (b) along the vertically aligned arrows in figure 4(a) (averaged over five lines); roman numbers refer to arrows in figure 4(a). In addition, the cyan curve in (b) shows an EBIC profile taken across the pn-heterojunction of a different lamella with $80 \mathrm{kV}$ instead of $300 \mathrm{kV}$ in a region without any dislocations. Note that the EBIC magnitude for $80 \mathrm{kV}$ is higher due to a different beam current and inelastic scattering cross section.

profile (i) in figure 5(a). The apparently stronger recombination behaviour of dislocation II can be partially explained by the slightly higher excess carrier diffusion length of STNO [14]. However, the intersection of the profiles in figure 5(a) shows that the dislocation induced recombination below the core is stronger than at its actual position, which is not the case for dislocation I. A possible explanation for this behaviour is the interplay between the dislocation itself and the electric field of the space charge region of the pn-heterojunction. The latter is supposed to be maximal at the chemical interface and to decay monotonously resulting in a potentially smaller charge carrier separation and hence an effectively larger influence of the dislocation induced recombination. The black and yellow vertical profiles in figure 5(b) confirm indeed that the main part of the reduced EBIC signal is located inside the space charge region expected to extend at least $10 \mathrm{~nm}$ into the STNO [14]. 
Finally, we would like to emphasise the reproducibility of the experiment and an experimental evidence that beam spread effects of the electron probe resulting from (multiple) scattering can be ruled out as the resolution-limiting effect in these experiments: The cyan curve in figure 5(b) shows the EBIC profile across the pn-heterojunction taken from another lamella with an acceleration voltage of $80 \mathrm{kV}$ instead of $300 \mathrm{kV}$. Since any beam broadening effects are expected to strongly depend on the acceleration voltage, the strong matching of the shape of the two measured profiles provides evidence that beam spread is negligible as well as that different lamellae lead to identical results within experimental error.

\section{Summary and Conclusion}

We have reported an experimental setup achieving a resolution of at least a few nanometers by means of scanning transmission EBIC (STEBIC) investigations offering the possibility to map the recombination and transport behaviour of transition metal oxide perovskites with relevant physical quantities in the 1-10 $\mathrm{nm}$ range. The spatial extension of STEBIC signals is limited by sample properties such as the excess carrier diffusion lengths of the materials rather than by the size of the generation volume resulting from electron scattering. This is evidenced by showing that the shape of the resulting STEBIC profiles is independent of the chosen acceleration voltage.

Applying this technique to misfit dislocations, we have shown that the position of the dislocation core can have a tremendous impact on the resulting excess carrier recombination. In fact, in contrast to interfacial dislocations, buried dislocations shifted inside the STNO show an enhanced EBIC decrease and, in addition, a medium range recombination effect. The obvious effect of different bulk recombination properties of the two materials on the dislocation recombination behaviour has been discussed qualitatively. Nevertheless, the underlying mechanisms are not yet fully understood.

A sufficiently precise model has to describe the concerted action of (i) the presence of the pnjunction's space charge region, (ii) potential surface recombination at the two lamella surfaces, (iii) additional electric field below the lamella surfaces as a result of Fermi level pinning, and (iv) the formation of 'dead layers' due to sample preparation. The latter have been discussed to some extent in [19] and related to experimental results obtained on the PCMO/STNO system and a silicon pnjunction. Surface recombination in the absence of any electric field can be described in terms of a Green's function approach which has been evaluated numerically in [32] by placing a large number of mirror charges in order to satisfy the boundary conditions at the surfaces resulting in a quantitative description of EBIC signals obtained outside the charge collecting junction's space charge region. Other models considering effective excess carrier diffusion lengths or a surface recombination factor in the EBIC profile have been introduced in [33] and [34]. Furthermore, sophisticated and quantitatively proven models of excess carrier recombination at dislocations [35] assume excitations in the neutral bulk and cannot be readily adopted to the situation in our experiments. Hence, all existing models are based on the assumption of exciting the sample outside the electric field of the space charge region. A possibility to fill this gap is the employment of finite element simulations, which we are currently working on.

\section{Acknowledgements}

We thank Arne Ahrens for valuable discussions. Funding by the Deutsche Forschungsgemeinschaft (DFG) via the CRC 1073, project B02 and Z02, is gratefully acknowledged.

\section{References}

[1] van Santen J H and Jonker G H 1950 Physica 16337

[2] Bednorz J G and Müller K A 1986 Z. Physik B - Condensed Matter 64189

[3] von Helmolt R, Wecker J, Holzapfel B, Schultz L and Samwer K 1993 Phys. Rev. Lett. 712331

[4] Sawa A, Fujii T, Kawasaki M and Tokura Y 2005 Appl. Phys. Lett. 86112508

[5] Röder H, Zang J and Bishop A R 1996 Phys. Rev. Lett. 761356

[6] Zener C 1951 Phys. Rev. 82403 
[7] Anderson P W and Hasegawa H 1955 Phys. Rev. 100675

[8] Gennes P G 1960 Phys. Rev. 118141

[9] Abdi-Jalebi M et al. 2018 Nature 555497

[10] Mildner S, Hoffmann J, Blöchl P, Techert S and Jooss C 2015 Phys. Rev. B 92035145

[11] Dagotto E 2013 Nanoscale Phase Separation and Colossal Magnetoresistance (Berlin: Springer Science \& Business Media)

[12] Yamada S, Arima T H, Ikeda H and Takita K 2000 Journal of the Physical Society of Japan 69 1278-1281

[13] Raiser D, Mildner S, Ifland B, Sotoudeh M, Blöchl P, Techert S and Jooss C 2017 Adv. Energy Mater. 71602174

[14] Ifland B, Hoffmann J, Kressdorf B, Roddatis V, Seibt M and Jooss C 2017 New J. Phys. 19 063046

[15] Shockley W and Queisser H J 1961 Journal of Applied Physics 32510

[16] Bi C Z, Ma J Y, Yan J, Fang X, Zhao B R, Yao D Z and Qiu X G 2006 Journal of Physics: Condensed Matter 182553

[17] Ifland B, Peretzki P, Kressdorf B, Saring P, Kelling A, Seibt M and Jooss C 2015 Beilstein Journal of Nanotechnology 61467

[18] Leamy H J 1982 Journal of Applied Physics 53 R51

[19] Donolato C 1981 phys. stat. sol. (a) 65649

[20] Bethe H 1930 Annalen der Physik 4325

[21] Cabanel C, Brouri D and Laval J 2006 The European Physical Journal 34107

[22] Peretzki P, Ifland B, Jooss C and Seibt M 2017 Phys. Status Solidi RRL 111600358

[23] Chin A K, Temkin H and Roedel R J 1979 Appl. Phys. Lett. 34476

[24] Goldstein J I 1977 Scanning Electron Microscopy 1977315

[25] Falkenberg M A, Schuhmann H, Seibt M and Radisch V 2010 Review of Scientific Instruments 81063705

[26] Ifland B, Hoffmann J, Kramer T, Scherff M, Mildner S and Jooss C 2015 J. Nanomaterials 31

[27] Chandrasena R U et al. 2017 Nano Lett. 17794

[28] Hoffmann J, Moschkau P, Mildner S, Norpoth J, Jooss C, Wu L and Zhu Y 2014 Mater. Res. Express 1046403

[29] Jirak Z, Krupicka S, Simsa Z, Glouha M and Vratislav S 1985 Journal of Magnetism and Magnetic Materials $\mathbf{5 3} 153$

[30] Glazer A M 1972 Acta Crystallographica Section B 283384

[31] Saucke G, Norpoth J, Jooss C, Su D and Zhu Y 2012 Phys. Rev. B 85165315

[32] Tan C C, Ong V K and Radhakrishnan K 2013 Prog. Photovolt: Res. Appl. 21986

[33] Donolato C 1983 Appl. Phys. Lett. 43120

[34] Ong V K S, Phang J C H and Chan D S H 1994 Solid-State Electronics 371

[35] Kveder V V, Kittler M and Schröter W 2001 Phys. Rev. B. 63115208 


\subsection{Extraction of Bulk Diffusion Lengths and Sur- face Layer Properties}

As mentioned in chapter 3, EBIC can be employed to measure the bulk diffusion length of underlying materials as long as the lateral expansion of the interaction volume of the impinging electron beam is smaller than resp. comparable with the latter and surface recombination effects can be disentangled from the bulk behaviour. In this section, the confined interaction volume using STEM-EBIC will be exploited to quantify the dead layer thickness, the reduced surface recombination velocity $(s=S / D)$, as well as the nanoscale bulk diffusion length of STNO according to Eq. (3.3). Furthermore, a qualitative increase of the EBIC profile extension in RP-PCMO in comparison with PCMO will be demonstrated and the effect of delocalised inelastic interactions of the electron beam with the sample will be discussed.

Fig. 7.4 shows an ADF-STEM overview image of the wedge-shaped PCMO-STNO lamella presented in the previous section (left) with the scanning region marked by a green rectangle as well as the thickness with respect to the horizontal scanning position (right) determined via Eq. (3.1) with $\lambda=110 \mathrm{~nm}$ [162] and extracted at the interface. Please note that the thickness in the vertical direction differs by less than $10 \mathrm{~nm}$ within the domain of finite EBIC signals. Consequently, vertical line profiles across the interface
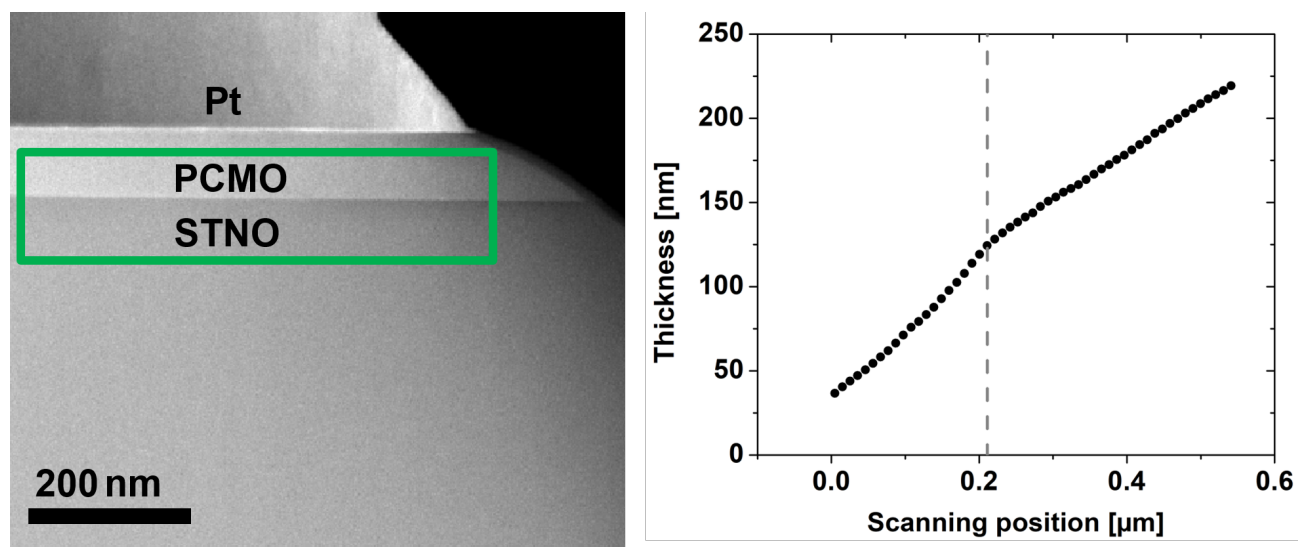

Figure 7.4: ADF-STEM overview of the wedge-shaped PCMO-STNO lamella with the scanning area used for EBIC marked by a green rectangle (left) as well as the thickness extracted at the interface with respect to the horizontal scanning position (right). The edge of the final thinning window is marked with a dashed grey line. 
will be considered as representatives of junctions with homogeneous thickness. Furthermore, it is important to mention that the kink in the thickness profile shown in Fig. 7.4 corresponds to the edge of the final thinning window.

In order to extract thickness dependent decay lengths of the EBIC profiles, the approach presented in [121] in the context of simulated EBIC profile is transferred to the experiment, i.e. the inner-most point of the fitting interval is adjusted such that the standard deviation of the decay length given by the fit is minimal. An exemplary EBIC profile with the optimal position marked is shown in Fig. A.13 and the evolution of resulting decay lengths as well as standard deviations with respect to the position of the inner-most point is given in Fig. A.14 and A.15. Consistent with the findings in [121], a systematic overestimation of the decay length occurs if the inner-most point is approaching the central part of the profile, i.e. the SCR. Importantly, the fit results also tend to slighty higher values if only a few data points above the noise level are included. However, in the case of STNO, the standard deviation is minimal in a range where only small changes of the resulting decay length are observed and the inner-most point yielding the best fit lies a few nanometer outside the expected SCR with an extension on the STNO side of approximately $12 \mathrm{~nm}$ following Eq. (2.18) and using $\epsilon_{n}=340$ [166], $\epsilon_{p}=30$ [167], and $V_{\mathrm{D}}=0.77 \mathrm{~V}$ [16]. In contrast, in the case of PCMO, no region with virtually constant decay lengths exist, i.e. the EBIC profiles exhibit a transient behaviour and cannot be described accurately by an exponential decay, and the standard deviation of the fit is minimal if the inner-most point is in close proximity to the chemical junction. Consequently, the discussion will be restricted to STNO first. Please note that the origin of the vertical scanning direction was set to the intersection of 0.5 and the relative STNO contribution in the low-loss EEL spectra given by the solution of

$$
\min _{a} \int_{15 \mathrm{eV}}^{45 \mathrm{eV}}\left(s_{x}(E)-a \cdot s_{\mathrm{STNO}}(E)-(1-a) \cdot s_{\mathrm{PCMO}}(E)\right)^{2} d E
$$

Here, $s_{x}, s_{\text {STNO}}$, and $s_{\text {PCMO }}$ are the EEL spectra recorded at scanning position $x$ as well as in the STNO resp. PCMO far from the interface and normalised by their total number of counts. A plot of the optimal values of $a$ with respect to the scanning position as well as exemplary EELS spectra are given in Fig. A.16 resp. A.17 revealing a sharpness of the junction of approximately $2 \mathrm{~nm}$. Consequently, signal variations on the order of $2 \mathrm{~nm}$ apart from the interface should be taken with care when discussing driftdiffusion properties as they might be simply caused by material changes. 

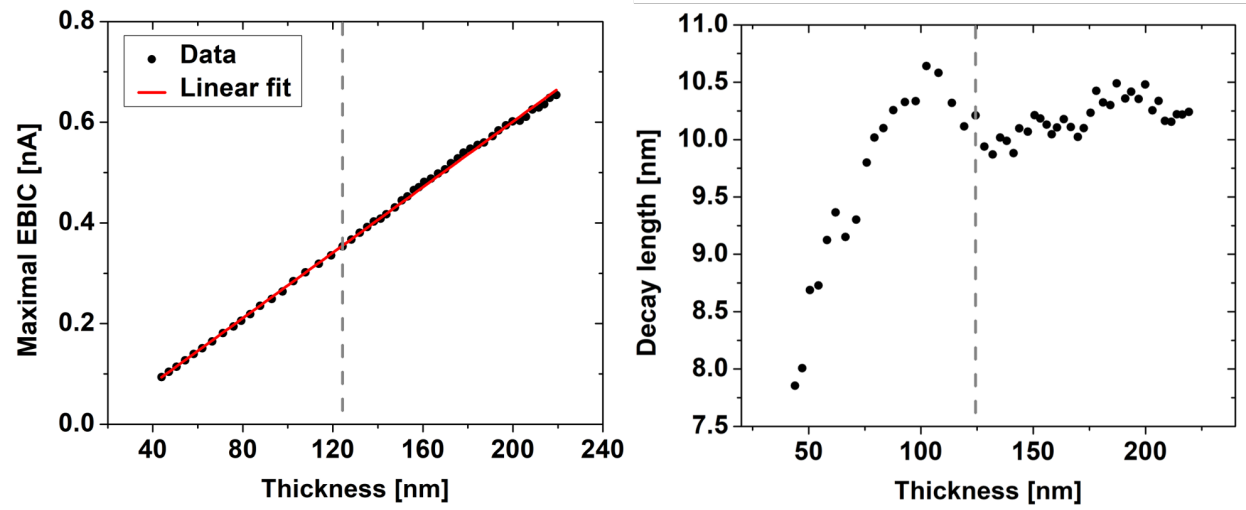

Figure 7.5: Thickness dependence of the maximal EBIC value of the wedgeshaped PCMO-STNO lamella including a linear fit (left) as well as the decay length of STNO (right). The edge of the final thinning window is marked by the dashed grey line.

Fig. 7.5 shows the thickness dependence of the maximal EBIC values (left) as well as the extracted decay lengths of STNO (right) with the edge of the thinning window marked by dashed grey lines. The maximal EBIC value is linearly increasing with the thickness, intersects the thickness axis at 15.2(6) nm and shows no significant changes at the edge of the final thinning window. In contrast, the latter does have an impact on the decay length in the STNO suggesting that the collection efficiency in the field-immersed SCR is more robust than in the neutral material. Furthermore, the decay length of the STNO fits qualitatively to the behaviour expected from Eq. (3.3). Due to influences of the edge of the thinning window as well as the fact that the thickness gradient of approximately 0.6 presented in Fig. 7.4 is rather high and the assumption of homogeneity on the scale of lateral diffusion might be unjustified, the saturation level of the STNO decay length of 10.3(2) nm (averaged between $180 \mathrm{~nm}$ and $220 \mathrm{~nm}$ ) shall be the only quantitative figure extracted from this dataset and identified as the corresponding bulk diffusion length.

In order to study the thickness range in which the STNO decay length changes sensitively more closely and suppress lateral heterogeneities even further, the (RP-PCMO)-STNO lamella was thinned with a $3^{\circ}$ wedge angle resulting in a ten times smaller thickness gradient of approximately 0.06 and consequently a change in thickness of less than a nanometer on the scale of the STNO bulk diffusion length. The related maximal EBIC values as well 

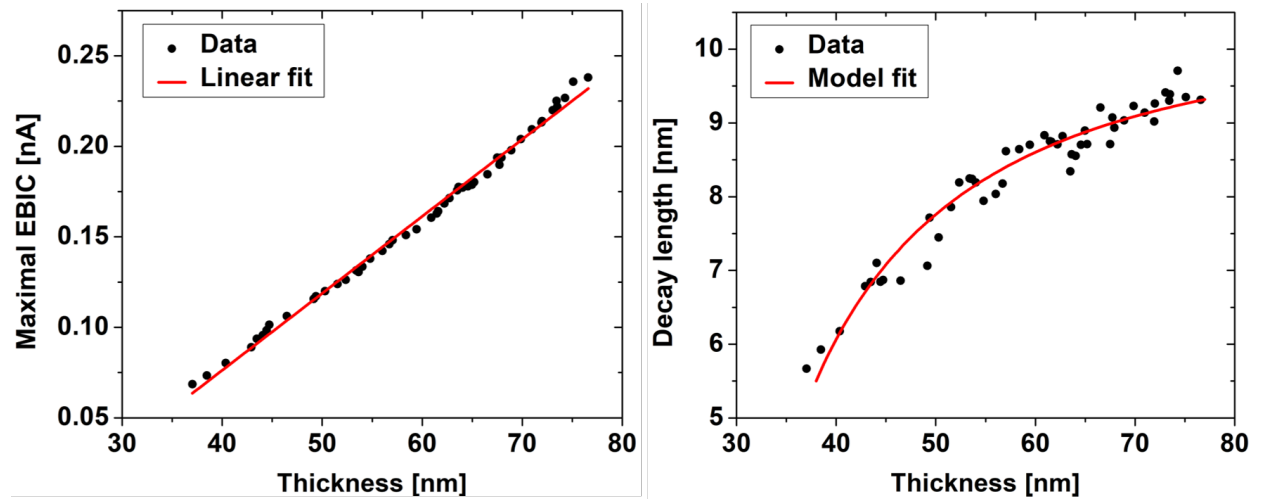

Figure 7.6: Thickness dependence of the maximal EBIC value of the wedgeshaped (RP-PCMO)-STNO lamella including a linear fit (left) as well as the decay length of STNO (right). The red curve shows a fit of Eq. (3.3) to the experimental data.

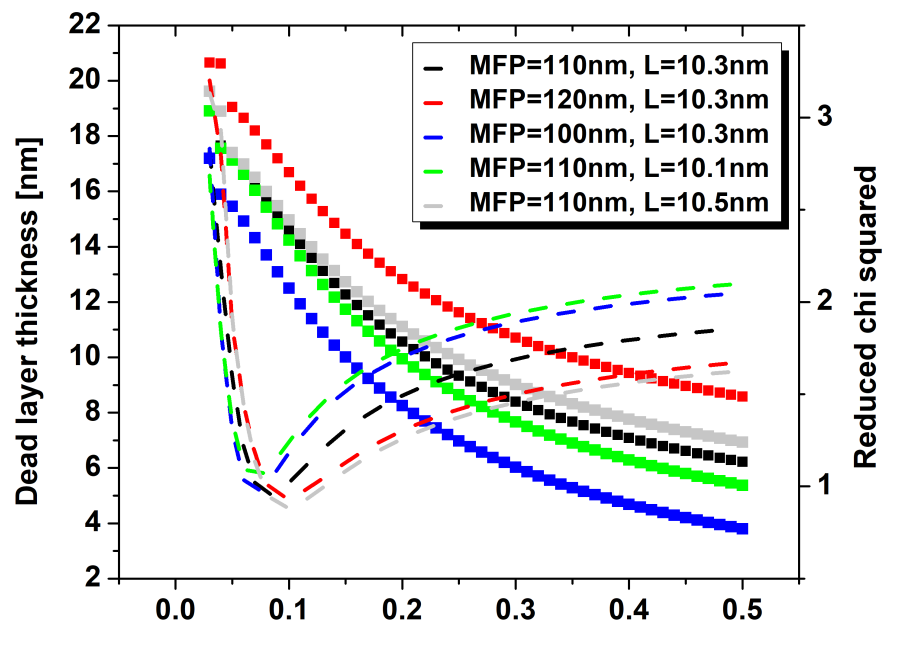

Reduced surface recombination velocity $[1 / \mathrm{nm}]$

Figure 7.7: Optimal dead layer thickness $t_{\mathrm{d}}$ as well as the corresponding residual reduced $\chi^{2}$ for the fit of Eq. (3.3) to the STNO decay lengths shown in Fig. 7.6 for different choices of $s$, the inelastic mean free path, as well as the bulk diffusion length. 
as extracted decay lengths of STNO are given in Fig. 7.6. Furthermore, a fit of Eq. (3.3) to the experimental data is shown in red, which has been obtained in the following manner: The previously observed bulk diffusion length of $L=10.3(2) \mathrm{nm}$, i.e. the saturation level in Fig. 7.5 (right), is fixed and for a sweep of the reduced surface recombination velocity $s=S / D$ the dead layer thickness $t_{\mathrm{d}}$ yielding the lowest reduced $\chi^{2}$ is determined. This approach is chosen to visualise the effect of the uncertainties of fixed experimental parameters, i.e. the inelastic mean free path (MFP) used for the thickness determination as well as the bulk diffusion length. The standard deviation of the extracted decay lengths was set to $0.2 \mathrm{~nm}$ in accordance with the one observed in the saturated regime in Fig. 7.5 and the resulting values of $t_{\mathrm{d}}$ and $\chi^{2}$ are presented in Fig. 7.7 as functions of $s$ for different choices of the inelastic MFP as well as $L$, i.e. variations within realistic experimental accuracies. Remarkably, for all used parameters, the reduced $\chi^{2}$ obtains a sharp minimum with optimal values around unity indicating that the experimental curve is sensitive to $s$ and no overfitting occurs. Within the presented variations of the inelastic MFP and $L$, the dead layer thickness resp. reduced surface recombination velocity can be determined to $t_{\mathrm{d}}=15(1) \mathrm{nm}$ resp. $s=0.08(2) \mathrm{nm}^{-1}$. Compared to the results in [29], both the bulk diffusion length as well as the dead layer thickness is smaller, which is consistent with the herein higher $\mathrm{Nb}$ doping concentration, i.e. an expectedly lower mobility and screening length, as well as the decreased ion acceleration voltage of $5 \mathrm{kV}$ instead of $30 \mathrm{kV}$ during final thinning. Interestingly, the dead layer thickness of STNO is significantly larger than half the onset of the maximal EBIC values, i.e. the intersection with the thickness axis, mentioned earlier indicating once more that the field-immersed SCR is less prone to surface effects. This finding could possibly be related to the simulation results in [86] showing differently strong influences of surface charges in the SCR compared with the neutral material. In fact, this would imply that depleted regions - being modelled as dead layers in this work emerge due to surface charging.

Lastly, the focus shall be moved again to RP-PCMO and PCMO. As mentioned previously, the strong dependence of extracted decay lengths on the fitting interval suggests that the EBIC profile within the films cannot be modelled accurately by an exponential function. In anticipation of so far disregarded effects like a finite travel distance of excited plasmons (or other generated quasi-particles) which eventually decay to electron-holepairs, near-field inelastic interactions, e.g. a coupling to evanescent interface 
states, superimposed exponential functions were fitted to the EBIC tails as well as combinations including a linear transient, which could be caused by a finite sheet resistance impeding the transport of the created secondary electron current. However, in none of the cases, the strong dependence of extracted parameters on the fitting interval could be circumvented and an analytical expression describing the profile shape is yet to be found. In fact, heterogeneities in the film close to the interface, e.g. a change in octahedral tilts due to epitaxial strain [168], could serve as an alternative explanation for the observed profile shape. Still, considering the decay length of an exponential fit to the EBIC tail as a measure of its extension while keeping the fitting interval constant, a qualitative comparison between RP-PCMO and PCMO can be inferred from the data presented in Fig. 7.8. In both cases, a slight increase of the decay length is observed for small thicknesses saturating around $60 \mathrm{~nm}$ to $70 \mathrm{~nm}$. Interestingly, the saturation value in RP-PCMO is slightly higher than in PCMO being consistent with the enhanced photovoltaic activity of the former [27]. However, whether this finding is related to charge ordering at room temperature as reported in the cited reference remains unclear and should be studied in future experiments, e.g. by EBIC at elevated temperatures in the disordered phase.

\subsection{In-situ Biased STEM-EBIC of PCMO $(x=$ $0.34)$}

Given the electric contacts of the TEM lamellas, external voltages can be applied in order to manipulate the rectifying junctions while collecting EBIC data as demonstrated in this section. Fig. 7.9 shows resulting exemplary 


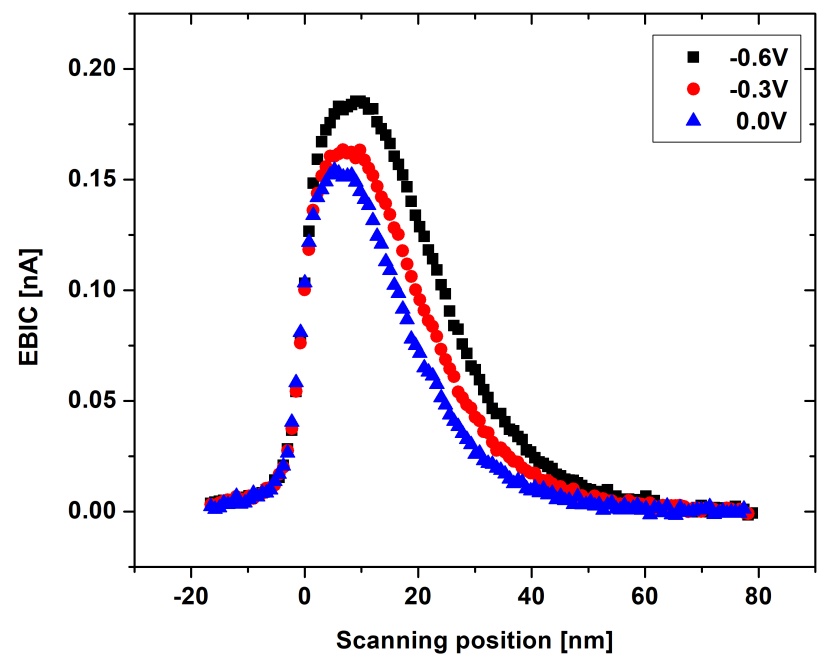

Figure 7.9: Exemplary EBIC profiles of a $70 \mathrm{~nm}$ thick, uniformly thinned PCMO-STNO junction at different reverse bias voltages. The direct current caused by the external power supply was subtracted.
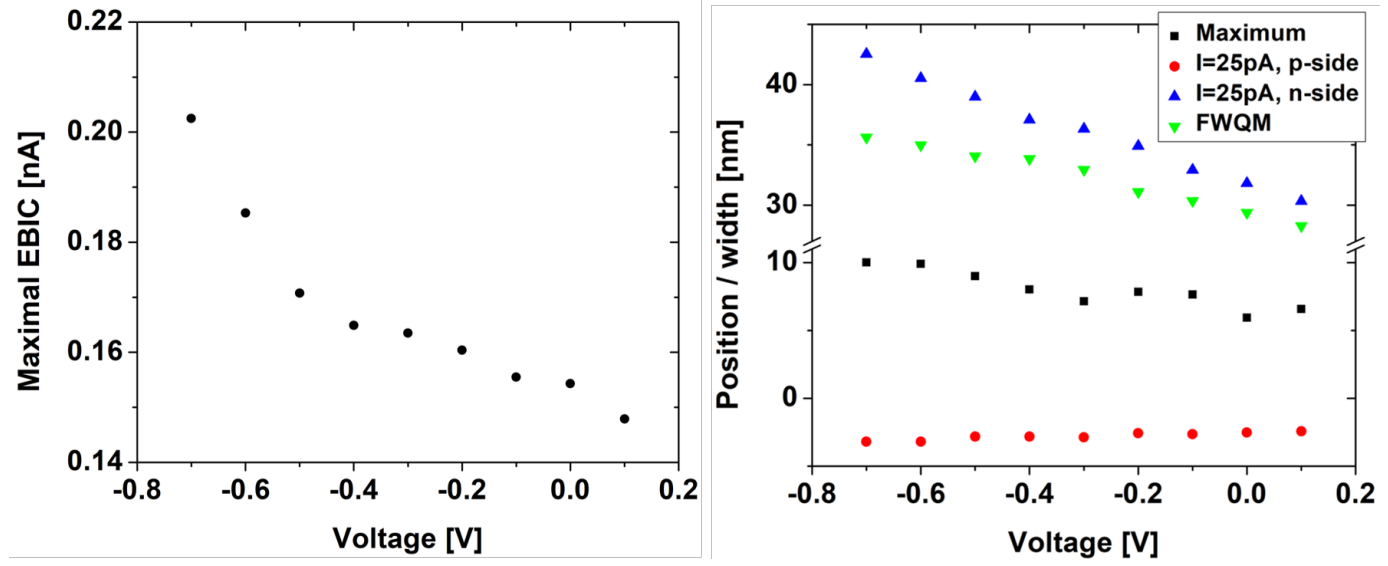

Figure 7.10: Voltage dependence of the maximal EBIC (left) as well as the position of the maxima and intersections with $I=25 \mathrm{pA}$ on both respective sides (right). 
profiles of a uniformly thinned PCMO-STNO lamella with an approximate thickness of $70 \mathrm{~nm}$ at different applied reverse bias voltages. Importantly, the direct current evoked by the external power supply, i.e. the constant value far from the junction, is already subtracted from the profiles. Please note that the interface, i.e. the origin of the scanning position, was determined following the procedure presented in the previous section and that the STNO contributions to the low-loss EEL spectra presented in Fig. A.16 are independent of the bias voltage suggesting that the generation remains unchanged.

However, the external stimulus does have an impact on the resulting EBIC signals leading to generally higher EBIC maxima as well as wider profiles with increasing reverse bias. Importantly, no significant change in the decay lengths is observed implying that predominantly the SCR is manipulated. Fig. 7.10 shows the voltage dependence of the maximal EBIC (left) and the corresponding position as well those of the intersections of the profiles with $I=25 \mathrm{pA}$ (right). Furthermore, the full width quarter maximum (FWQM) is presented. The maximal EBIC shows concave behaviour for small reverse bias voltages indicating a saturating collection efficiency as it would be expected while approaching unity. In contrast, a drastic superlinear increase is observed below $-0.4 \mathrm{~V}$, which could be explained by impact ionisation, i.e. an avalanche effect caused by the strong field in the SCR. Interestingly, the maximum position as well the profile intersections do not show a significant change in behaviour below $-0.4 \mathrm{~V}$ and can be welldescribed by linear functions. The ratio of the slopes of the blue and red curve (being relatively insensitive to the chosen intersection level) obtains an absolute value of $0.06(1)$, which is on the order of $N_{\mathrm{D}} / N_{\mathrm{A}}=0.03$, i.e. the ratio of the SCR extension on the respective sides following Eq. (2.18). However, no functional relation between such intersection levels and the SCR width exists so far and the low curvature of the extracted positions raises doubt whether they differ solely by a constant offset. In fact, assuming for a moment that the SCR edges are voltage independent, an altered collection efficiency inside the latter would still affect the height of the EBIC profiles and lead to changes in the extracted positions. As an alternative approach, the FWQM was extracted to suppress the mentioned effect revealing a concave behaviour. Still, a fit to Eq. (2.18) plus a constant offset sensitively depends on the included data points as well as the intersection level, i.e. whether the FWQM or full width $w$ maximum values with $w \neq 0.25$ are used. Consequently, more thorough analytical resp. numerical modelling is needed to reliably extract characteristic points of the SCR from resulting EBIC profiles and should be part of future studies. 


\subsection{STEM-EBIC in a Textured Silicon Solar Cell}

So far, the presented STEM-EBIC signals were exclusively recorded in highlydoped TMOPs with inherently strong bulk recombination rates leading to screening and diffusion lengths on the nanoscale. However, the increased spatial resolution compared with SEM-EBIC also facilitates the observation of classical semiconductors as it has been demonstrated in the context of boron diffusion into a textured silicon solar cell in the following preprint [169] posted on arXiv (arxiv.org/abs/2109.00586v1) - the author's contributions are given in the main text. All contents are reprinted in their original form with permission and the reader is kindly asked to check for eventually updated cross-links in case of future journal publication.

By combining the experiment with FE-EBIC as well as boron diffusion simulations, a sub $0.1 \mathrm{ppm}$ isoconcentration line of boron in a non-planar geometry was successfully mapped on the nanoscale. Furthermore, enabled by the tremendous advances in two-dimensional EBIC simulations achieved by D. A. Ehrlich [121], an effective recombination model projecting along the electron propagation direction could be employed to simulate entire signal maps. The corresponding recombination coefficients were fixed in the neutral regions leading to an overall good agreement with the experiment and a precise localisation of the chemical interface with equal donor and acceptor concentrations. Importantly, due to the low doping of the $n$-type substrate, the mentioned isoconcentration line is well below the resolution limit of complementary techniques such as secondary ion mass spectrometry [170]. Furthermore, it is worth mentioning that the remaining differences between simulation and experiment at the edges of the SCR should contain valuable information about the Fermi level dependence of involved recombination processes and the existing dataset might be very useful to develop strategies to close the gap between simulation and experiment as it was recorded on a homojunction with comparatively small strain effects and material heterogeneities. 


\title{
Tracing the boron diffusion into a textured silicon solar cell by combining boron diffusion simulation with experimental and simulated scanning transmission electron beam induced current
}

\author{
Tobias Meyer, ${ }^{1}$ David A. Ehrlich, ${ }^{1}$ Peter Pichler, ${ }^{2,3}$ Valeriya Titova, ${ }^{4,5}$ \\ Christoph Flathmann, ${ }^{1}$ Jan Schmidt, ${ }^{4,5}$ and Michael Seibt ${ }^{1, *}$ \\ ${ }^{1} 4$ th Institute of Physics - Solids and Nanostructures, \\ University of Goettingen, Friedrich-Hund-Platz 1, 37077 Göttingen, Germany \\ ${ }^{2}$ Fraunhofer Institute for Integrated Systems and Device Technology IISB, Schottkystrasse 10, 91058 Erlangen, Germany \\ ${ }^{3}$ Universität Erlangen-Nürnberg, Lehrstuhl für Elektronische Bauelemente Cauerstrasse 6, 91058 Erlangen, Germany \\ ${ }^{4}$ Institute for Solar Energy Research Hamelin (ISFH), Am Ohrberg 1, 31860 Emmerthal, Germany \\ ${ }^{5}$ Institute of Solid-State Physics, Leibniz Universitaet Hannover, Appelstrasse 2, 30167 Hannover, Germany
}

\begin{abstract}
The light absorption of [001] grown single-crystalline silicon wafers can be enhanced by chemical etching with potassium hydroxide resulting in a pyramid-like surface texture. Alongside this advantageous property in the context of solar energy conversion, the surface roughness leads to drawbacks as well, e.g. difficulties in measuring diffusion behaviour of dopants in the heterogeneous structure. In this paper, we employ experimental and simulated scanning transmission electron beam induced current in combination with the simulation of boron diffusion to map a sub $0.1 \mathrm{ppm}$ isoconcentration line underneath the textured surface on the nanoscale. In order to account for surface recombination, an effective two-dimensional model projecting the system along the electron beam propagation direction is used in the finite elements EBIC simulation. We find a good agreement to the experimental data and discuss future strategies to eliminate remaining deviations inside the space charge region.
\end{abstract}

\section{INTRODUCTION}

The theoretical limit for the efficiency of single junction crystalline silicon solar cells, also known as the ShockleyQueisser limit, amounts to approximately 30 percent [1]. Accounting only for transmission of sub-bandgap photons and thermalisation of hot carriers, this value is still significantly higher than practically achieved performances being detoriorated by e.g. enhanced excess charge carrier recombination at the contacts or reflection of the incident light $[2,3]$. Thus, electrically and chemically passivated as well as textured, strongly absorbing surfaces are highly desirable. A low-cost processing step to achieve a pyramid-like surface texture and consequently high absorption of [001] silicon wafers is chemical etching [3]. Nevertheless, the surface texture can interfere with subsequent preparation steps such as the diffusion of dopants to form the solar cell's emitter layer. Unfortunately, classical methods to determine doping profiles like electrochemical capacitance voltage (ECV) measurements resp. secondary ion mass spectrometry (SIMS) become difficult in the presence of textured surfaces due to limited lateral resolution [4] resp. a decreasing elemental sensitivity for decreasing sputtering volumes [5], i.e. increasing spatial resolution. In addition, SIMS yields the elemental concentrations independent of their electrical activity being of major interest in the context of device fabrication. Alternatively, the diffusion of dopants [6], in particular boron $[7,8]$, in

\footnotetext{
*Electronic address: mseibt@gwdg.de
}

silicon was studied extensively on planar samples to determine corresponding diffusion parameters which can be used subsequently as input parameters for the simulation of heterogeneous structures. In fact, an enhanced boron mobility in the case of an oxidizing ambience was found in [7]. The entire path of extracting diffusion parameters in a 1D model from planar samples and applying them numerically to $2 \mathrm{D}$ pyramid-like textured surfaces is presented in [9]. Additionally, electron beam induced current (EBIC) measurements are presented to verify the qualitative shape of the simulated isoconcentration lines.

In this work, we demonstrate how scanning transmission EBIC in combination with boron diffusion simulation can be employed to quantitatively map a sub $0.1 \mathrm{ppm}$ iso-concentration line underneath a textured single-crystalline silicon solar cell on the nanoscale. As reported in [10-14], the spatial resolution of EBIC in transmission mode is superior to its conventional counterpart since the electron beam does not get spread significantly due to scattering in the sample. Clearly, surface effects become more dominant in a thin, electron transparent lamella, which is addressed by comparing the experimental data to finite element simulations (FEM) in an effective two-dimensional model. We find a good agreement between experimental and simulated maps and remaining remaining deviations in the space charge region (SCR) are thoroughly discussed.

\section{SAMPLE PREPARATION AND INSTRUMENTATION}

A detailed description of the preparation process of the silicon solar cell can be found in [15]. Nevertheless, 
a brief summary of the main steps related to the boron diffusion process conducted in a Tempress Systems TS81004 furnace shall be given:

1. Heat-up: The furnace temperature is increased from $700^{\circ} \mathrm{C}$ to $941^{\circ} \mathrm{C}$ using a ramping speed of $10^{\circ} \mathrm{C} / \mathrm{min}$ in an inert ambience.

2. Deposition: Keeping the temperature in the furnace stabilized, $\mathrm{N}_{2}$ is lead through a liquid $\mathrm{BBr}_{3}$ bubbler held at $20^{\circ} \mathrm{C}$ leading to a $\mathrm{B}_{2} \mathrm{O}_{3}$ flow which is subsequently mixed with $\mathrm{N}_{2}$ and $\mathrm{O}_{2}$.

3. Drive-in: The flow through the bubbler is switched off while all other parameters remain unchanged for further $20 \mathrm{~min}$.

4. Post-oxidation: The $\mathrm{N}_{2}$ and $\mathrm{O}_{2}$ gas flows are reduced and the temperature is held constant for further $60 \mathrm{~min}$.

5. Cool-down: In an inert ambience, the temperature is decreased by $-10^{\circ} \mathrm{C} / \mathrm{min}$ and the sample carrier ultimately retracted at $805^{\circ} \mathrm{C}$.

In addition to the potassium hydroxide treated textured wafer, a planar reference sample was processed simultaneously and investigated by four point probe (4PP) measurements subsequently to determine the sheet resistance of the resulting boron doped layer in the homogeneous case.

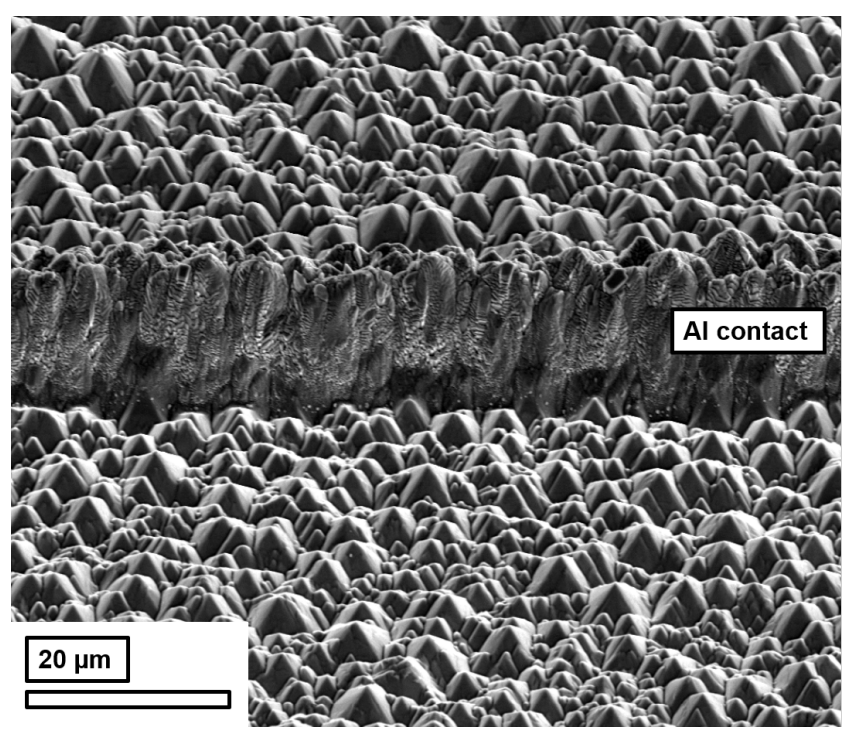

FIG. 1: SEM overview of the pyramid texture of the solar cell including an aluminium front contact. The surface is inclined by 45 degrees.

An SEM overview of the the textured surface including an aluminium front contact is shown in Fig. 1. An electron transparent lamella was extracted from the aluminium covered area using an FEI Nova NanoLab Dual Beam focused ion beam. The acceleration voltage for

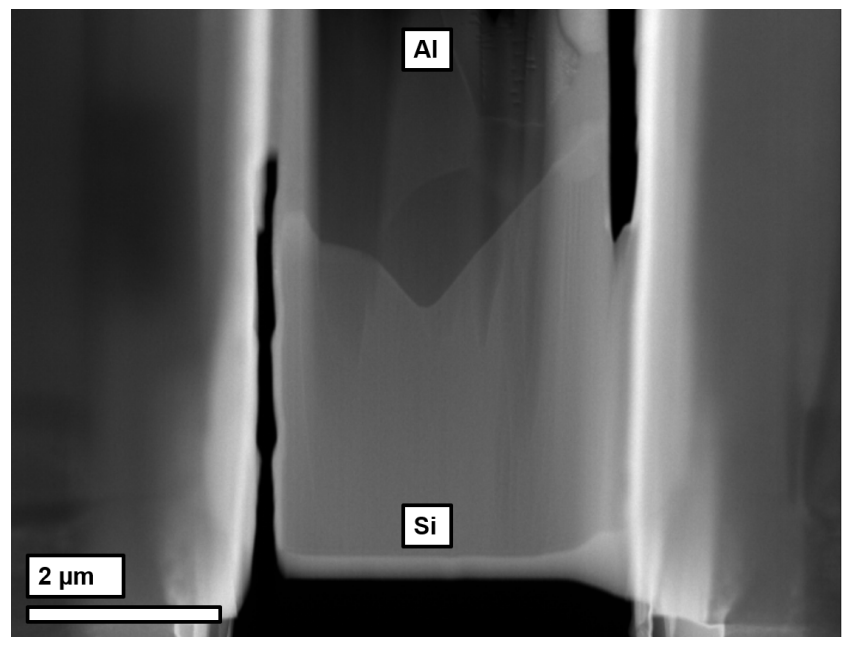

FIG. 2: ADF STEM overview of the [110] oriented lamella including a trench between two pyramids in the central region. Vertical cuts have been performed to prevent short circuits across the investigated area.

the final thinning step was decreased from $30 \mathrm{kV}$ to $5 \mathrm{kV}$ in order to minimize beam damage. An annular darkfield (ADF) STEM overview of the resulting lamella is shown in Fig. 2. The vertical cuts have been performed during FIB preparation to prevent short circuits across the electrical junction due to redeposited material. To allow for electrical contacting inside the scanning transmission electron microscope (STEM), a DENSsolutions Lightning D7+ holder was used. All STEM experiments were performed inside an FEI Titan80-300 operated at $300 \mathrm{kV}$. The beam current was set to $42 \mathrm{pA}$ and an acceptance semi-angle of $39 \mathrm{mrad}$ was used for electron energy loss spectroscopy (EELS) employing a Gatan Quantum 965 ER. The short circuit current was converted to an output voltage with a Stanford Research Systems SR570 and subsequently fed to the AD converter of the Gatan DigiScan II scan unit controlling the electron beam during the acquisitions.

\section{EXPERIMENTAL RESULTS}

The central part of the lamella shown in Fig. 2 including a trench between two pyramids was investigated simultaneously by ADF STEM resp. EBIC yielding the results presented in Fig. 3(a) resp. (b). Clearly, the domain of finite EBIC signals lies well below the textured surface and the shape is comparably smooth. In order to illustrate the shape of the EBIC signal across the junction, a normalized profile averaged over the four left-most columns in Fig. 3(b) is shown in Fig. 7. Including only points below 0.25 to exclude effects of the space charge region, the decaying tails of the profile on each respective side were fitted to an exponential function yielding a decay length of the EBIC signal of $83 \mathrm{~nm}$ resp. $136 \mathrm{~nm}$ on the p- resp. n-side. Correcting for the approx- 
imately $40^{\circ}$ inclination of the surface, this translates to effective diffusion lengths of $63 \mathrm{~nm}$ resp. $103 \mathrm{~nm}$. Obviously, these values are well below those expected in bulk material indicating the strong influence of surface recombination which will be discussed later in detail. However, due to the homogeneous thickness in the EBIC domain of 1.76(4) mean inelastic free paths (determined by EELS following Malis' method [16]) which translates to approximately $342(7) \mathrm{nm}$ [17], relative thickness effects on the excess charge carrier recombination can be neglected.

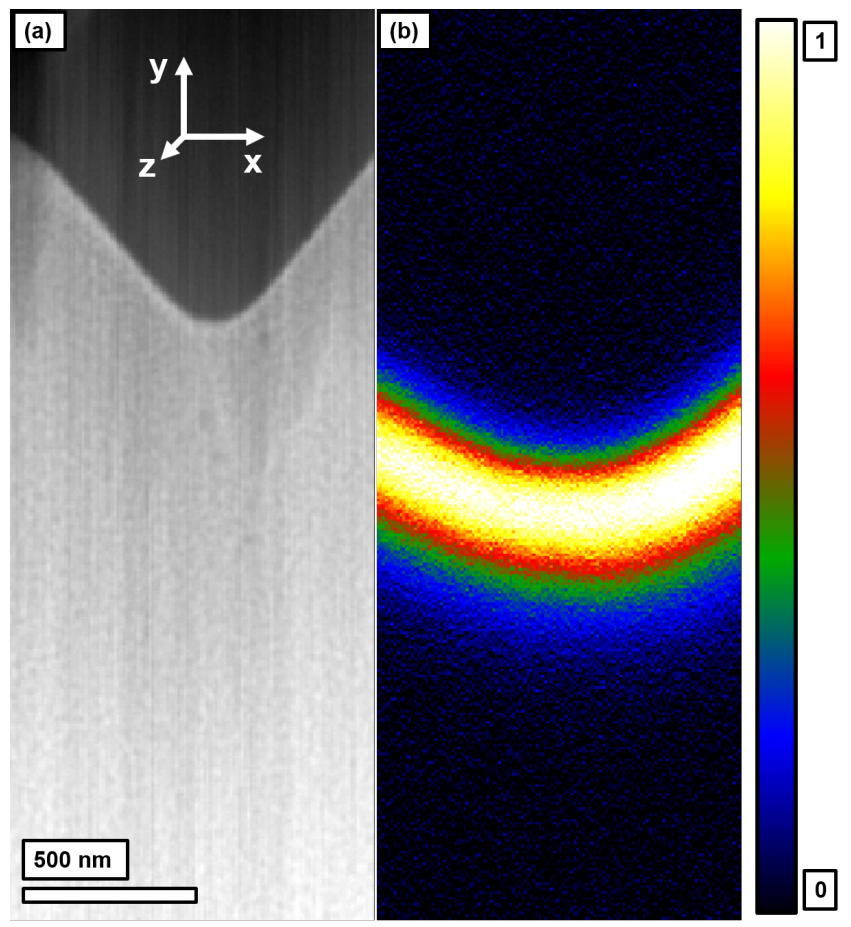

FIG. 3: (a) ADF STEM image of the central region of the lamella and (b) the corresponding EBIC map normalized to its maximum. The spatial directions used throughout the paper are indicated in (a) and the origin is set to the upper left corner in all following figures.

\section{BORON DIFFUSION SIMULATION}

While sufficiently accurate models exist for the diffusion of boron in silicon, this is neither the case for the growth of boron glasses nor for the diffusion of boron in the glass or for its segregation into silicon. In lack of more detailed knowledge, the main process steps are modeled as follows: Following [18], the partial pressures of $\mathrm{O}_{2}$ and $\mathrm{B}_{2} \mathrm{O}_{3}$ under the given conditions in the furnace are calculated yielding the growth of a thin partially liquid boron glass. The doping of the growing glass with boron is simulated using a Dirichlet boundary condition at the interface between the ambience and the glass. After the boron source is switched off, a negligible evaporation of boron into the furnace is assumed during the process steps drive-in, post-oxidation and cool-down. It has to

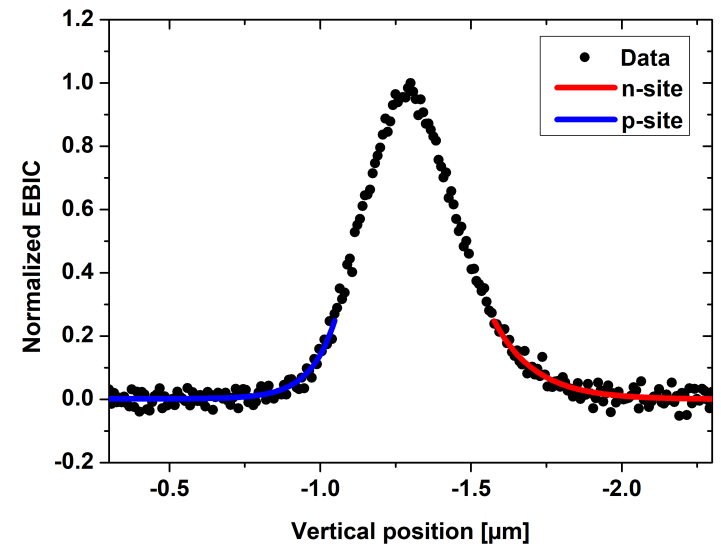

FIG. 4: Vertical EBIC profile averaged over the four left-most columns in Fig. 3(b) as well as exponential fits of the profile's tails on the $\mathrm{n}$ - and $\mathrm{p}$-side, respectively. Only values below 0.25 have been considered during fitting.

be noted, though, that simulations with diffusion-limited evaporation and an adapted boron concentration at the interface to the ambience during the deposition step lead to very similar boron profiles. Particularly during the post-oxidation step, the boron diffuses deeply into the silicon but segregates also into the growing oxide glass. This results in a retrograde boron profile in the silicon with a maximum concentration approximately $0.12 \mu \mathrm{m}$ below the surface.

For the numerical implementation, Sentaurus Process of Synopsys, Version Q-2019.12, was used with AdvancedCalibration and the two-phase segregation model for boron at the silicon-silicon dioxide interface. Within the simple modeling approach taken, the boron concentration at the oxide surface during the deposition step has a main influence on the final sheet concentration of boron in the silicon and thus on its sheet resistance. A second uncertainty results from the enhancement of the boron diffusion during all process steps in oxidizing atmosphere. Established models exist for the dry oxidation of silicon with low dopant concentrations in the oxide. However, the oxide glass growing here is highly doped and partially liquid which should result in considerably less strain in the glass. Following the arguments of [19], the different stress state should result also in a different injection of self-interstitials and thus oxidation enhancement.

In order to fix the boron concentration at the oxide surface during deposition as well as the degree of oxidation enhancement, two experimental observations are used: Firstly, the sheet resistance of $102 \Omega /$ sq obtained by the $4 \mathrm{PP}$ measurements on the planar reference sample. Secondly, the position of the chemical junction, assuming that a boron concentration of $3.5 \times 10^{15} \mathrm{~cm}^{-3}$ coincides with the column-wise EBIC maximum underneath the tip of the trench in Fig. 3(a). Both experimen- 


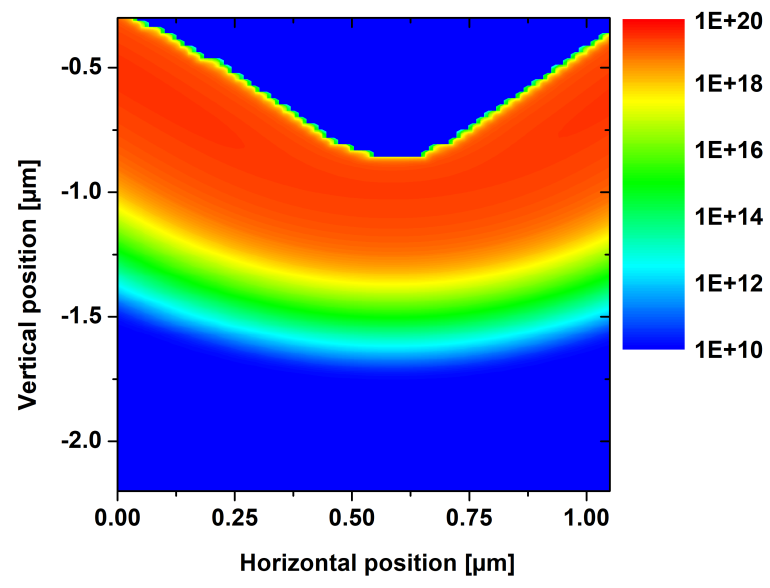

FIG. 5: Simulated two-dimensional boron concentration underneath the surface texture shown in Fig. 3(a). The numbers in the colour-code are given in $\mathrm{cm}^{-3}$.

tal findings are met using an oxide surface concentration of $N_{\mathrm{B} \text {, surface }}=1.55 \times 10^{22} \mathrm{~cm}^{-3}$ during the deposition and an OED scaling factor of about 0.34 leading to the twodimensional boron distribution underneath the textured surface shown in Fig. 5. For this figure, to avoid boundary effects, the surface topography was linearly extrapolated on the left and right side by each $500 \mathrm{~nm}$ during the simulation and Neumann boundary conditions were assumed for the diffusion equations.

\section{NUMERICAL EBIC MODEL}

To simulate the drift diffusion behaviour of excess charge carriers, a 2D model (omitting the electron propagation direction, i.e. $z$ ) with three variables is used: The electrostatic potential $\phi$ as well as the electron and hole concentrations $n$ and $p$. In addition, following parameters are needed: The ionized acceptor and donor concentrations $N_{\mathrm{a}}^{-}$and $N_{\mathrm{d}}^{+}$, the relative permittivity $\epsilon_{\mathrm{r}}$, the electron and hole mobilities $\mu_{\mathrm{e}}$ and $\mu_{\mathrm{h}}$, the intrinsic charge carrier concentration $n_{\mathrm{i}}$ as well as the excess charge carrier generation and recombination rates $g$ and $r$. The set of partial differential equations that have to be solved is given by Poisson's equation (1) and the stationary continuity equations including sinks and sources for electrons (2) and holes (3):

$$
\begin{array}{r}
-\vec{\nabla}\left(\epsilon_{0} \epsilon_{\mathrm{r}} \vec{\nabla} \phi\right)=e\left(N_{\mathrm{d}}^{+}-N_{\mathrm{a}}^{-}+p-n\right), \\
0=\frac{\mathrm{d} n}{\mathrm{~d} t}=-\vec{\nabla}\left(\mu_{\mathrm{e}} n \vec{\nabla} \phi-\frac{k_{\mathrm{B}} T}{e} \mu_{\mathrm{e}} \vec{\nabla} n\right)+g-r, \\
0=\frac{\mathrm{d} p}{\mathrm{~d} t}=-\vec{\nabla}\left(-\mu_{\mathrm{h}} p \vec{\nabla} \phi-\frac{k_{\mathrm{B}}}{e} T \mu_{\mathrm{h}} \vec{\nabla} p\right)+g-r .
\end{array}
$$

Please note that the two parenthesized terms on the right side of equation (2) and (3) correspond to the drift respectively diffusion of charge carriers and that the Boltzmann approximation has been used to express the latter with mobilities. All necessary steps to deduce the given equations can be found e.g. in [20]. The values of the constant parameters used in the simulations are summarized in Tab. I. The concentration of ionized donors is held constant at $3.5 \times 10^{15} \mathrm{~cm}^{-3}$, which correpsonds to the wafer's resistivity of $1.4 \Omega \mathrm{cm}$, whereas the ionized acceptor concentration is is given by the boron density presented in Fig. 5.

The generation rate $g$ due to the electron beam is modelled as a gaussian, where the mean value represents the position of the electron beam. The standard deviation was set to $10 \mathrm{~nm}$ and the amplitude to $8.87 \times 10^{25} \mathrm{~cm}^{-3} \mathrm{~s}^{-1}$. The recombination rate $r$ is expressed by the following term which is motivated by a single Shockley-Read-Hall ( $\mathrm{SRH}$ ) recombination centre [21] (but omits reemission from the trap state):

$$
r=\left(n p-n_{\mathrm{i}}^{2}\right) /\left(\tau_{\mathrm{h}} n+\tau_{\mathrm{e}} p\right) .
$$

This definition aims to describe an effective lifetime dominated by defects states due to the surfaces. The recombination rate is enhanced in the space charge region where carrier concentrations are low as it is the case for trap levels well inside the bandgap. Reemission is excluded as it demands for a third input parameter, i.e. the trap energy level, which is unknown in the first place. In contrast, $\tau_{\mathrm{h}}$ and $\tau_{\mathrm{e}}$ are defined by the experimental tails in the neutral regions shown in Fig. 4 where either $n$ or $p$ become clearly dominating majority carriers and set to $\tau_{\mathrm{h}}=1.988 \times 10^{-11} \mathrm{~s}$ and $\tau_{\mathrm{e}}=7.130 \times 10^{-13} \mathrm{~s}$. Similarities to existing approaches in the literature as well as consequences of this simplification will be discussed in detail in the next section.

The boundary conditions at the EBIC contacts in $y$ direction are chosen to be Dirichlet conditions with $\phi=0$, $n=n_{i}^{2} / N_{\mathrm{B} \text {,surface, }}$ and $p=N_{\mathrm{B} \text {,surface }}$ at the p-side and $\phi=k_{\mathrm{B}} T / e \log \left(N_{\mathrm{d}}^{+} N_{\mathrm{B} \text {,surface }} / n_{i}^{2}\right), n=N_{\mathrm{d}}^{+}$, and $p=n_{i}^{2} / N_{\mathrm{d}}^{+}$at the n-side. The EBIC current is evaluated at the $\mathrm{n}$-side contact. The boundary conditions in $x$ direction are chosen to be zero flux Neumann conditions, i.e. the $x$ component of the gradient of $\phi, n$, and $p$ are set to zero.

For the numerical implementation, COMSOL Multiphysics, Version 5.4., was used. In order to avoid boundary effects, the surface was (in accordance with the boron diffusion simulation) linearly extrapolated on the left and right side by each $500 \mathrm{~nm}$ during the simulation.

\section{COMPARISON OF EXPERIMENT AND SIMULATION}

The previous three sections do not only present the experimental results and used numerical models, but also reflect their dependencies on each other as the position 
TABLE I: Parameters in equation (1)-(4) used for the FEM simulations.

\begin{tabular}{|c|c|c|c|c|c|c|c|}
\hline Parameter & $\epsilon_{r}$ & $\mu_{\mathrm{e}}$ & $\mu_{\mathrm{h}}$ & $T$ & $n_{i}$ & $\tau_{\mathrm{h}}$ & $\tau_{\mathrm{e}}$ \\
\hline Value & 11.7 & $1100 \mathrm{~cm}^{2} \mathrm{~V}^{-1} \mathrm{~s}^{-1}$ & $200 \mathrm{~cm}^{2} \mathrm{~V}^{-1} \mathrm{~s}^{-1}$ & $300 \mathrm{~K}$ & $9.802 \times 10^{9} \mathrm{~cm}^{-3}$ & $1.988 \times 10^{-11} \mathrm{~s}$ & $7.130 \times 10^{-13} \mathrm{~s}$ \\
\hline
\end{tabular}

of the column-wise experimental EBIC maximum below the surface tip was used to fix the OED scaling factor in the boron diffusion simulation on which the numerical EBIC model was subsequently based on. Consequently, it is important to check for the model's consistency.

The experimental and simulated EBIC maps normalized to their maxima are shown in Fig. 6(a) and (b). Generally, the shape and position of the respective signals agree quite well, but small deviations exist. For the sake of a clearer presentation, exemplary vertical line profiles across the surface tip are shown in Fig. 7 together with the modulus of the electric field in equilibrium obtained from the FEM simulations. Firstly, it is worth mentioning that the EBIC signal is finite outside the SCR allowing for observations in the neutral semiconductors. However, while the EBIC shapes agree well in the field-free regions, the experimental signal tends to be smaller inside the space charge region with larger discrepancies on the n-side. The former finding is highly consistent with previous reports about effective diffusion lengths in field-free semiconductors accounting for bulk and surface recombination simultaneously [22, 23] and confirms the choice of $\tau_{\mathrm{h}}$ and $\tau_{\mathrm{e}}$ as corresponding effective lifetimes. In other words, in the neutral regions, the three-dimensional TEM lamella can be described by an effective two-dimensional model successfully. Nevertheless, the recombination rate inside the SCR, i.e. in the regime where the Fermi level crosses the energy level of potential trap states, is higher in the experiment and thus requires a refinement of the model given by Equation 4 and possibly an inclusion of surface charges. Indeed, it was shown numerically in [24] that charged surface states with trap levels below resp. above the mid-gap energy influence n-type resp. p-type material more strongly which might be related to the non-symmetric discrepancies in Fig. 7. Thus, a detailed analysis if and how sensitively the density of surface charges as well as the trap level of corresponding states can be inferred from EBIC measurements should be part of future studies.

After having discussed the impact of the excess charge carrier recombination model, the focus shall be moved back to the textured geometry and the resulting boron distribution. Figure 8 shows the aluminium-silicon interface (black), the experimental (red) and simulated (blue) column-wise EBIC maxima, as well as the chemical interface (green) resulting from the boron diffusion simulation. Generally, the three latter curves agree very well and only two significant deviations are observed: Firstly, the experimental EBIC maxima tend to lower values at the left which can be explained with the linear model extrapolation neglecting the finite curvature at the edge of the scanning area shown in Fig. 3(a) and is confirmed by

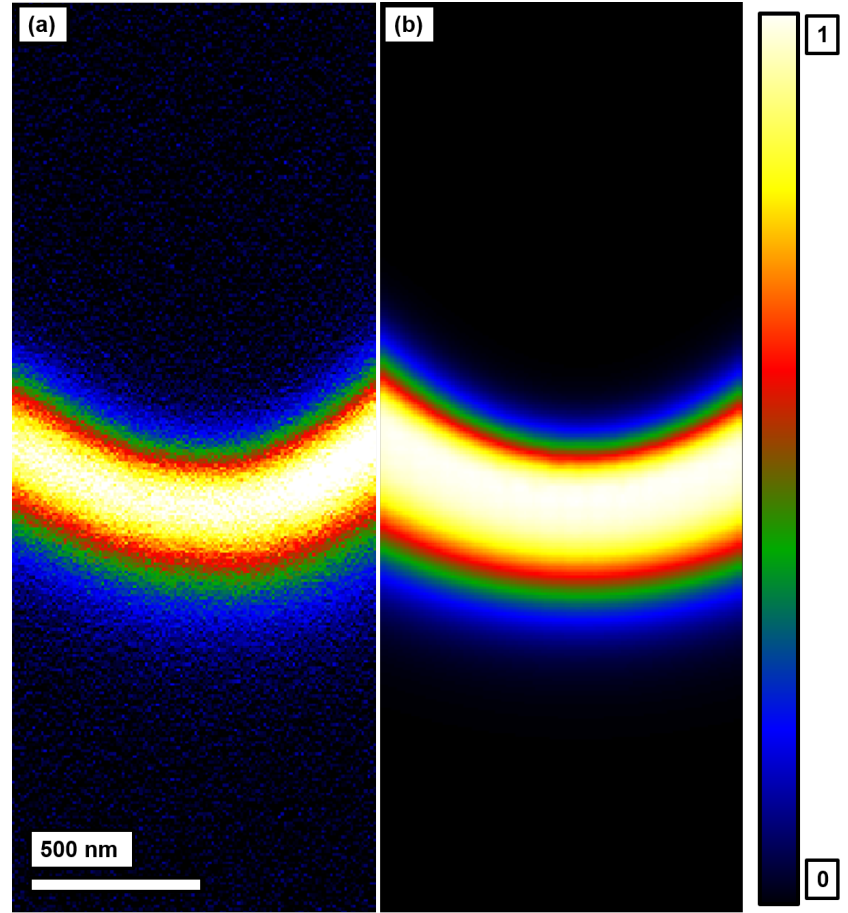

FIG. 6: (a) experimental resp. (b) simulated EBIC map normalized to their maxima.

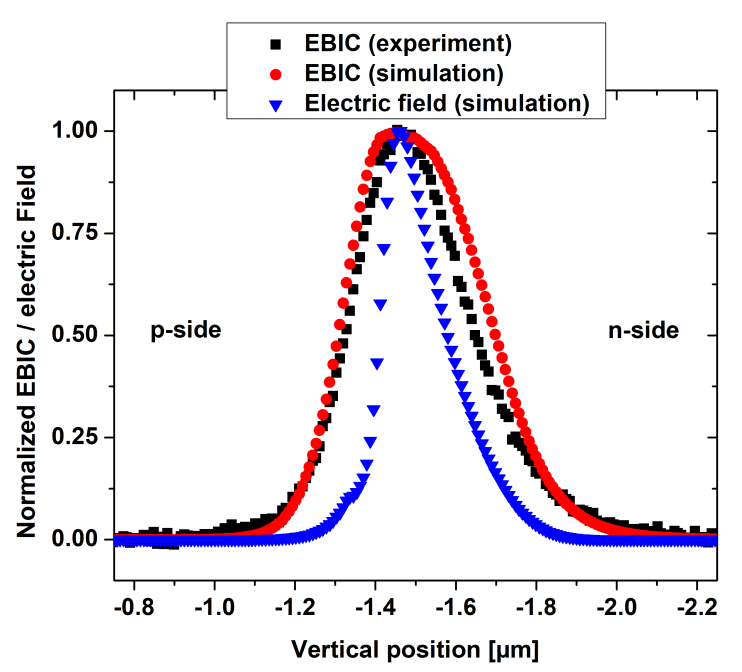

FIG. 7: Vertical profiles of the experimental (black) resp. simulated (red) normalized EBIC shown in Fig. 6(a) reps. (b) as well as the magnitude of the electric field (blue) resulting from the FEM simulation in equilibrium and normalized to its maximum. All profiles are taken across the tip of the surface texture. 
the absence of similar deviations at the right. Generally, such deviations could also emerge if heterogeneities lead to boron diffusion along the electron propagation direction which is neglected in the 2D simulation. Secondly, the curvature of the EBIC maxima is slightly below the chemical junction's which reflects the lateral diffusion of excess charge carriers and leads to a mean difference of $12(2) \mathrm{nm}$ between the simulated EBIC maxima and the chemical junction. In fact, the lateral diffusion gets relevant if the product of the effective diffusion length and the curvature of the chemical interface is non-negligible. Consequently, the strong influences of the surfaces are even beneficial for the sake of mapping the chemical interface, i.e. iso-concentration lines of diffusion processes, as they reduce the former. Furthermore, as indicated at the right edge, the simulated EBIC maxima shift slightly from the chemical interface (into the n-region) in the rather homogeneous case which is consistent with the findings in $[25,26]$. Thus, the comparably precise coincidence of all three curves underneath the tip could result from a compensation of oppositely acting effects, i.e. the mentioned shift as well as the lateral diffusion, and in the case of larger deviations a refined pinning of the chemical junction in the boron diffusion simulation had to be used. However, given this coincidence and including only the right half of the data (and thus excluding the inaccurate linear extrapolation on the left), the mean deviation between the experimental and simulated EBIC maxima amounts to only $2(3) \mathrm{nm}$.

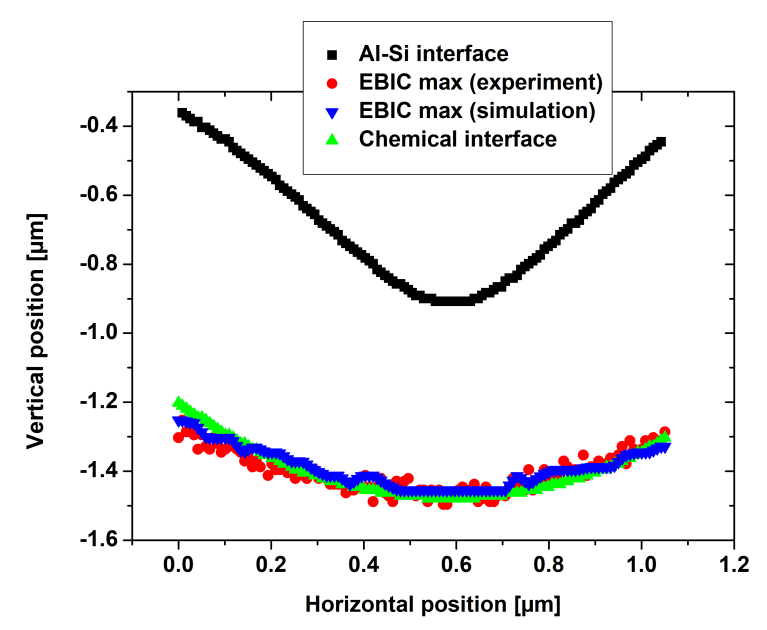

FIG. 8: Al-Si interface position (black) extracted from Fig. 3 (a) as well as the column-wise maximal values of the experimental (red) and simulated (blue) EBIC maps presented in Fig. 6(a) and (b). In addition, the position of the chemical interface (green) resulting from the simulated boron concentration is plotted.

\section{CONCLUSION}

In this paper, we presented a combined study of boron diffusion simulation as well as experimental and simulated scanning transmission EBIC in order to investigate the boron diffusion into a textured silicon surface. Generally, we found a good agreement between experimental and simulated EBIC by employing an effective twodimensional FEM model and fixing the recombination parameters of the SRH-motivated term in Equation 4 in the neutral regions.

Comparing vertical EBIC profiles (Fig. 7) as well as the column-wise EBIC maxima with the chemical junction resulting from the boron diffusion (Fig. 8), three major points have been illuminated leading to following conclusions:

1. Accuracy of the recombination model inside the SCR: Clearly, there are remaining deviation between expriment and simulation. This problem is well-known from SEM-based EBIC models and has predominantly been treated numerically $[24,25$, 27-29] where few experimental data points inside the SCR exist in the latter reference. Importantly, we have shown that signficant EBIC signals are obtained across the entire SCR and even outside. Given the low spread of the electron beam in the sample [30], STEM-EBIC should be well-suitable to quantitatively investigate the handshake of space charge and field-free regions in future studies even if the device size approaches the nanometer scale.

2. Modelling of the surface texture: The inaccurate linear extrapolation on the left side in Fig. 8 immediately leads to significant deviations between experiment and simulation. On the one hand side, this discrepancy could have been avoided by simply extrapolating the scanning range of the EBIC map with the texture seen in the overview shown in Fig. 2. On the other hand, the deviations show once more how sensitively EBIC reflects the boron diffusion process which is why we decided to keep the linear extrapolation.

3. Lateral diffusion of excess charge carriers: In combination with the supposed shift of the maxima to the n-side in the heterogeneous case, a mean difference of 12(2) nm between the simulated EBIC maxima and the chemical junction was observed which is in contrast to the mean deviations of $2(3) \mathrm{nm}$ between the experimental and simulated EBIC maxima significant. Nevertheless, as a consequence of the surfaces strongly suppressing lateral diffusion, this difference is rather small. Thus, thinning the specimen to electron transparence is even beneficial if direct conclusions about the boron concentration shall be drawn from the EBIC shape.

In summary, we have shown that STEM-EBIC allows for signal collection in- and outside the SCR of a tex- 
tured silicon solar cell and that the resulting currents can be modelled by means of classical semiconductor equations. In combination with the simulation of boron diffusion, the iso-concentration line $N_{\mathrm{B}}=3.5 \times 10^{15} \mathrm{~cm}^{-3}$ was mapped on the nanoscale which is (assuming generously a lateral resolution of $15 \mathrm{~nm}$ in our experiment) two orders of magnitude below the sensitivity theoretically achievable with SIMS (assuming an equivalent sputtering volume of $\left.10^{5} \mathrm{~nm}^{3}[5]\right)$.

\section{ACKNOWLEDGEMENTS}

We thank Jan Verhoeven for valuable discussions about the used electronic setup as well as Thomas Lehmann for cutting the solar cell with a wafer saw to reduce its size before lamella extraction. T.M, C.F., and
M.S. are funded by the Deutsche Forschungsgemeinschaft (DFG, German Research Foundation) - 217133147/SFB 1073, project B02. V.T. is funded via the PhD Scholarship Programme of the German Federal Environmental Foundation (DBU). J.S. acknowledges funding by the State of Lower Saxony.

\section{AUTHOR CONTRIBUTIONS}

T.M. conducted the experiments with support by C.F.; D.A.E. performed the EBIC and P.P. the boron diffusion simulations; V.T. prepared the macroscopic silicon solar cell under supervision of J.S.; T.M. wrote the manuscript under revision of M.S. and with contributions of P.P.; All authors read and agreed on the written paper and declare no conflicts of interest;
[1] W. Shockley and H. J. Queisser, Journal of Applied Physics 32, 510 (1961).

[2] V. Titova, D. Startsev, and J. Schmidt, in AIP Conference Proceedings (AIP Publishing LLC, 2018), vol. 1999, p. 040022.

[3] K. Singh, M. Nayak, S. Mudgal, S. Singh, and V. K. Komarala, Solar Energy 183, 469 (2019).

[4] Y. Komatsu, D. Harata, E. W. Schuring, A. H. Vlooswijk, S. Katori, S. Fujita, P. R. Venema, and I. Cesar, Energy Procedia 38, 94 (2013).

[5] D. McPhail, Journal of Materials Science 41, 873 (2006).

[6] P. Pichler, Intrinsic point defects, impurities, and their diffusion in silicon (Springer Science \& Business Media, 2012).

[7] D. A. Antoniadis, A. G. Gonzalez, and R. W. Dutton, Journal of the Electrochemical Society 125, 813 (1978).

[8] S. Mirabella, D. De Salvador, E. Napolitani, E. Bruno, and F. Priolo, Journal of Applied Physics 113, 3 (2013).

[9] F.-J. Ma, S. Duttagupta, K. D. Shetty, L. Meng, G. S. Samudra, B. Hoex, and I. M. Peters, Journal of Applied Physics 116, 184103 (2014).

[10] C. Cabanel, D. Brouri, and J. Laval, The European Physical Journal Applied Physics 34, 107 (2006).

[11] P. Peretzki, B. Ifland, C. Jooss, and M. Seibt, physica status solidi (RRL) - Rapid Research Letters 11, 1600358 (2017).

[12] T. Meyer, B. Kressdorf, J. Lindner, P. Peretzki, V. Roddatis, C. Jooss, and M. Seibt, in Journal of Physics: Conference Series (IOP Publishing, 2019), vol. 1190, p. 012009.

[13] M. Mecklenburg, W. A. Hubbard, J. J. Lodico, and B. Regan, Ultramicroscopy 207, 112852 (2019).

[14] A. P. Conlan, G. Moldovan, L. Bruas, E. Monroy, and D. Cooper, Journal of Applied Physics 129, 135701
(2021).

[15] V. Titova and J. Schmidt, AIP Advances 8, 125023 (2018).

[16] T. Malis, S. Cheng, and R. Egerton, Journal of Electron Microscopy Technique 8, 193 (1988).

[17] P. Potapov, Ultramicroscopy 147, 21 (2014).

[18] W. Barber, C. Boynton, and P. Gallagher, Journal of Chemical \& Engineering Data 9, 137 (1964).

[19] N. Cowern, Physical Review Letters 99, 155903 (2007).

[20] S. M. Sze and K. K. Ng, Physics of Semiconductor Devices (John wiley \& sons, 2006).

[21] L. E. Black, New perspectives on surface passivation: Understanding the Si-Al2O3 interface (Springer, 2016).

[22] L. Jastrzebski, J. Lagowski, and H. Gatos, Applied Physics Letters 27, 537 (1975).

[23] C. Donolato, Journal of Applied Physics 54, 1314 (1983).

[24] P. M. Haney, H. P. Yoon, B. Gaury, and N. B. Zhitenev, Journal of Applied Physics 120, 095702 (2016).

[25] M. Nichterwitz and T. Unold, Journal of Applied Physics 114, 134504 (2013).

[26] P. Peretzki et al., Physical Review Applied (submitted) (2020).

[27] C. C. Tan, V. K. Ong, and K. Radhakrishnan, Progress in Photovoltaics: Research and Applications 21, 986 (2013).

[28] B. Gaury and P. M. Haney, Journal of Applied Physics 120, 234503 (2016).

[29] J. E. Moore, C. A. Affouda, S. I. Maximenko, and P. Jenkins, Journal of Applied Physics 124, 113102 (2018).

[30] J. Goldstein, Scanning Electron Microscopy 1977 pp. 315-324 (1977). 


\subsection{Atomically Resolved STEM-EBIC}

Lastly in the context of STEM-EBIC, existing signal modulations on the atomic scale as well as possible origins shall be presented and discussed. Fig. 7.11 shows that an enhanced EBIC signal occurs in STNO[100], PCMO[110], and $\mathrm{Si}[110]$ if the electron beam is located at atomic columns. All images were rotated such that the rectifying junction is located to the left and the EBIC signals were normalised by the mean value of the presented scanning area. While an atomic modulation of a few percent can directly be inferred in the case of STNO and Si, the strong decay of the EBIC profile in PCMO leads to an additional horizontal gradient. However, vertical profiles with a constant distance to the interface reveal similar behaviour. So far, atomic modulations of beam induced currents have only been demonstrated for metals in the context of secondary electron emission electron beam induced current (SEEBIC) [123] in the absence of a charge separating junction.

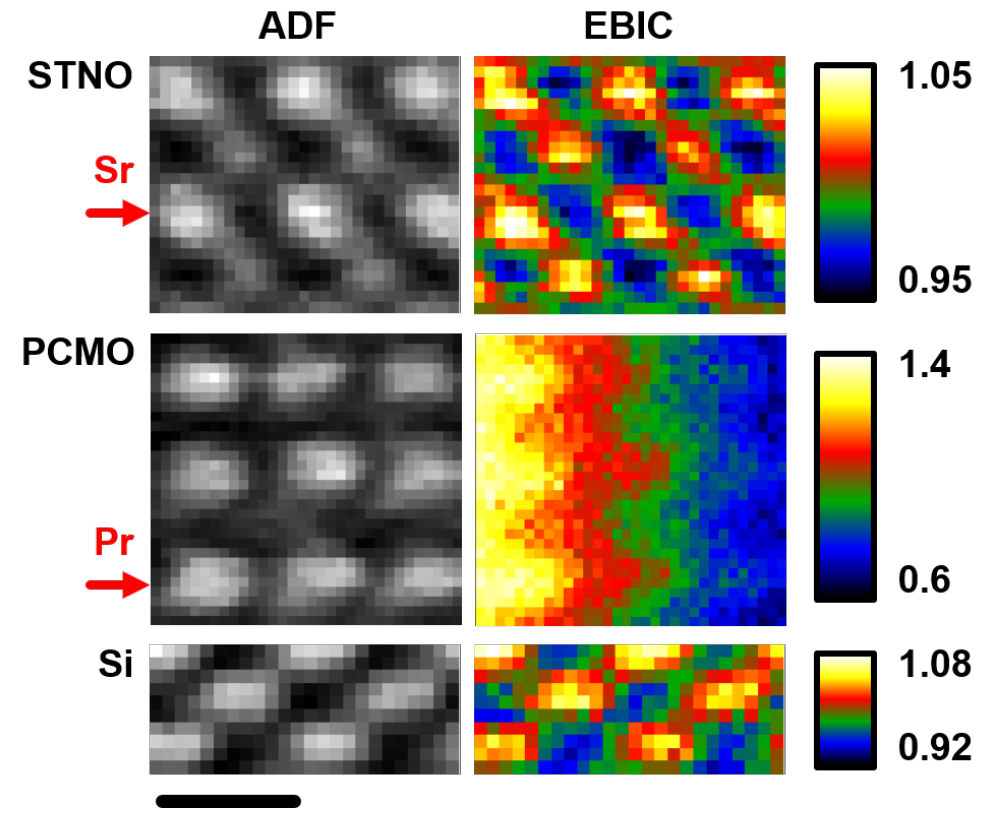

Figure 7.11: High-resolution ADF resp. STEM-EBIC maps of STNO[100] $(70 \mathrm{~nm}), \mathrm{PCMO}[110](70 \mathrm{~nm})$, and $\mathrm{Si}[110](125 \mathrm{~nm})$ showing signal modulations of a few percent. The EBIC maps were normalised by their mean value and all images were rotated such that the rectifying junction is located to the left. The scale bar corresponds to $0.4 \mathrm{~nm}$. 
In order to explore the underlying mechanisms causing the surplus signal, i.e. the difference at and between atomic sites, and how it is related to an internal junction, a correlative analysis including EEL spectra and the evolution of the modulation with respect to the interface position will be presented in this section.

Fig. 7.12 shows a reference EEL spectrum with a wide energy range of Si averaged over atomic sites and gaps (left) which has been normalised by the total sum of counts and multiplied with the beam current of $42 \mathrm{pA}$. Consequently, the scale corresponds to the current of primary electrons having undergone a certain energy loss and the integrated core-loss current (right) after power-law background subtraction represents an estimation of the SEEBIC current caused by core-loss events. Please note that a small fraction of the core-loss signal corresponds to excitations to bound final states [35] and that emitted SE could potentially be reabsorbed hence the extracted value should be considered as an upper limit of the core-loss related SEEBIC. For the sake of a comparison between atomic sites as well as the gaps in between, the domain of scanning positions is divided and the assignment of pixels to $\mathrm{Si}$ resp. gaps (as well as $\mathrm{Sr}, \mathrm{TiO}_{2}$, resp. gaps in the case of STNO) is described in Fig. A.18. Given this separation of the scanning area in the Si sample, the mean EBIC signal in the gaps amounts to $17.25(6) \mathrm{pA}$ and a surplus signal of $0.85(9) \mathrm{pA}$ is observed at the Si atomic sites. Furthermore, the cumulative deposited power, i.e.

$$
P(E)=\int_{0}^{E} s\left(E^{\prime}\right) E^{\prime} \mathrm{d} E^{\prime}
$$

is averaged over both domains where $s\left(E^{\prime}\right)$ is the EELS signal (scaled to a current as presented in Fig. 7.12) at energy loss $E^{\prime}$ (in units eV). The resulting curve averaged over gap positions (blue) as well as the surplus cumulative power at atomic sites (black) is shown in Fig. 7.13 (left).

Importantly, the scale differs by a factor of 0.049, i.e. the relative surplus EBIC allowing for the following comparison: Supposing that the additional EBIC signal is simply caused by a higher scattering cross section of all energy deposition channels, the two curves should align on the adjusted scales. However, this is not the case as the surplus power in the plasmon range, i.e. below the $\mathrm{Si} \mathrm{L}$-edge at $99 \mathrm{eV}$, is much lower. On the other hand, the power deposition through core-losses does scale with the surplus EBIC. Please note that technically, this proves solely a correlation between coreloss events and the additional EBIC but no causality and that low-energy 

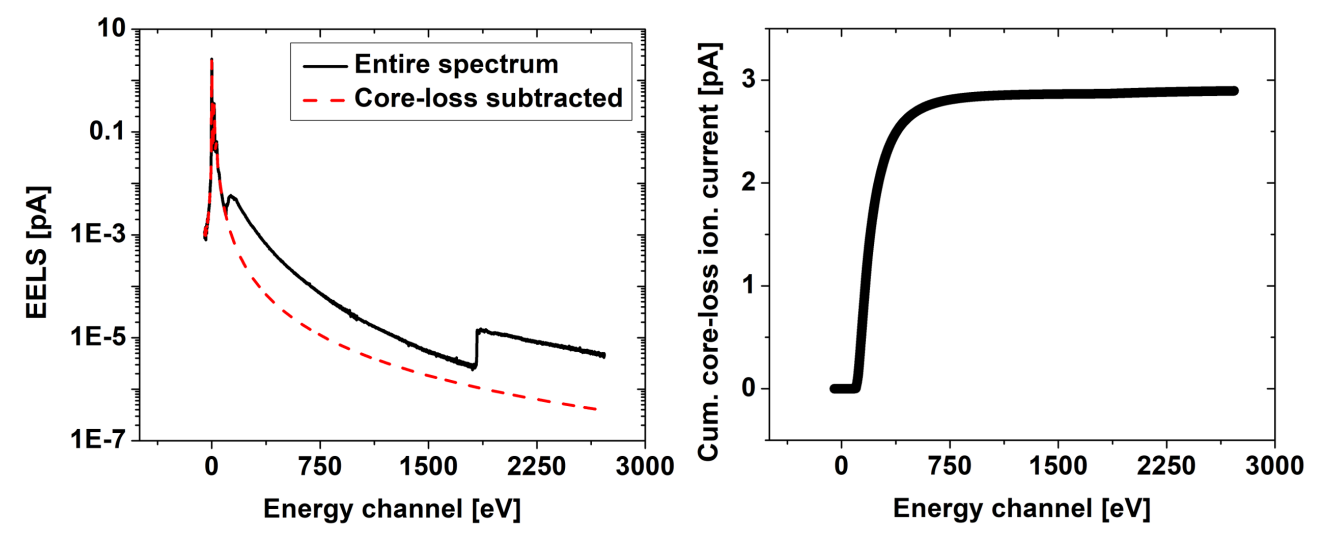

Figure 7.12: (Left) EEL spectrum of the investigated Si sample with and without core-loss contributions. Please note that the spectrum was first normalised by the total sum of counts and subsequently multiplied with the beam current of $42 \mathrm{pA}$. (Right) Cumulative core-loss ionisation current obtained by integrating solely the core-loss signal.
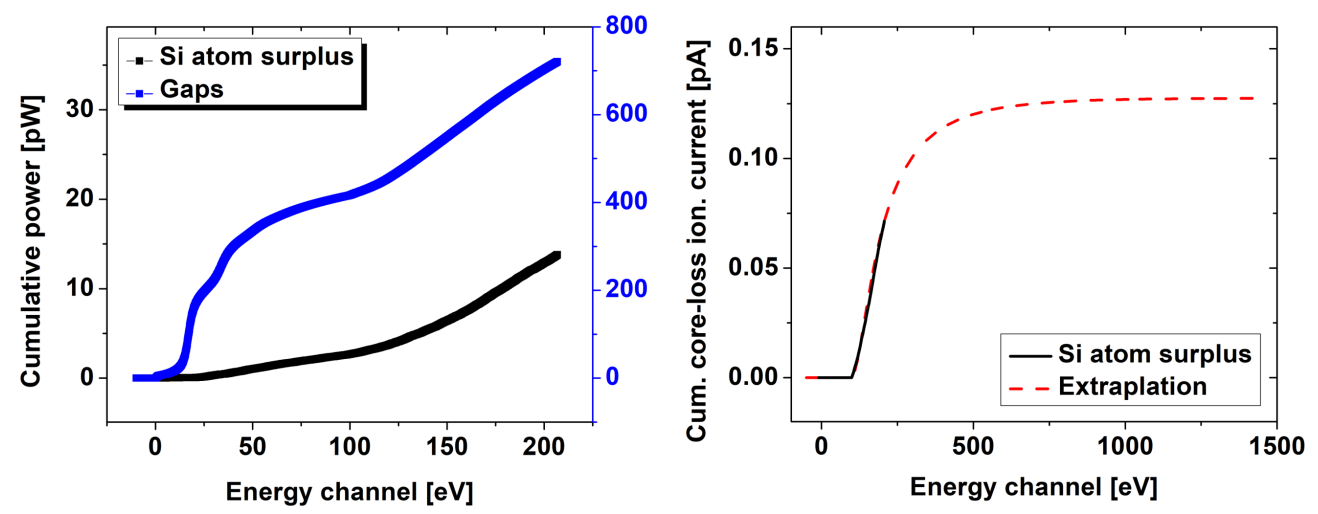

Figure 7.13: (Left) Cumulative power deposited by the electron beam averaged in the domain of gaps (blue scale) as well as the cumulative surplus power at the Si sites (black scale). Please note that the scales differ by a factor of 0.049 , i.e. the relative surplus EBIC signal at the atomic sites. (Right) Cumulative surplus ionisation current at the atomic sites as well as an extrapolation to higher energies based on the reference presented in Fig. 7.12 . 
excitations such as surface plasmons, possibly reabsorbed Cherenkov radiation, or the direct creation of excitons [35] might contribute to the atomic modulations as well. In fact, the cumulative power deposition yields only information about primary excitations and disregards the decay probability to excess charge carriers that can potentially be collected by the junction. Furthermore, when discussing core-losses, it is important to mention that an increased event rate leads to a trivial additional SEEBIC contribution as the material is constantly ionised. Still, by making use of the reference core-loss ionisation current presented in Fig. 7.12, the (upper limit) of this trivial SEEBIC contribution can be estimated to be approximately $0.12 \mathrm{pA}$ and thus significantly lower than the observed surplus EBIC of 0.85(9) pA as shown in Fig. 7.13 (right). Consequently, the atomic modulation must (at least partially) be caused by either cascades due to core-hole decays or the above mentioned low-energy excitations.

In order to investigate if and how such atomic modulations depend on the position with respect to the charge separating interface, several regions of interest as the one shown in Fig. A.18, each shifted by $5 \mathrm{~nm}$, were scanned in the STNO substrate of an approximately $70 \mathrm{~nm}$ thick PCMO-STNO lamella. After separating the scanning domain in $\mathrm{Sr}, \mathrm{TiO}_{2}$, and gap regions as described in Fig. A.18, the EBIC modulation $M$, defined as

$$
M=2 \cdot \frac{\left\langle I_{\text {atom }}\right\rangle-\left\langle I_{\text {gap }}\right\rangle}{\left\langle I_{\text {atom }}\right\rangle+\left\langle I_{\text {gap }}\right\rangle},
$$

was evaluated column-wise, i.e. for a constant distance to the interface and only if regions assigned to gaps and atomic sites exist in a column. Subsequently, $M$ was averaged for each region of interest yielding the spatial evolution shown in Fig. 7.14 (right). Please note that the error bars correspond to the standard deviation of $M$ in each region of interest and that the mean distance to the interface of the approximately $1.5 \mathrm{~nm}$ wide areas were assigned as scanning position. In addition, the mean EBIC is presented in Fig. 7.14 (left) yielding the familiar decay, which was already discussed extensively in the previous sections. Importantly, due to the column-wise evaluation of $M$, the transient of the collection efficiency as a function of the distance to the interface is cancelled out prior averaging. Interestingly, in both cases of $\mathrm{Sr}$ as well as $\mathrm{TiO}_{2}$ columns the EBIC modulation tends to smaller values as the charge separating interface is approached. Although remarkable, it is out of the scope of this thesis to establish a model describing the observed trend as well as confirming similar behaviour on different lamellas resp. sample systems reducing the likelihood of overlooked arti- 

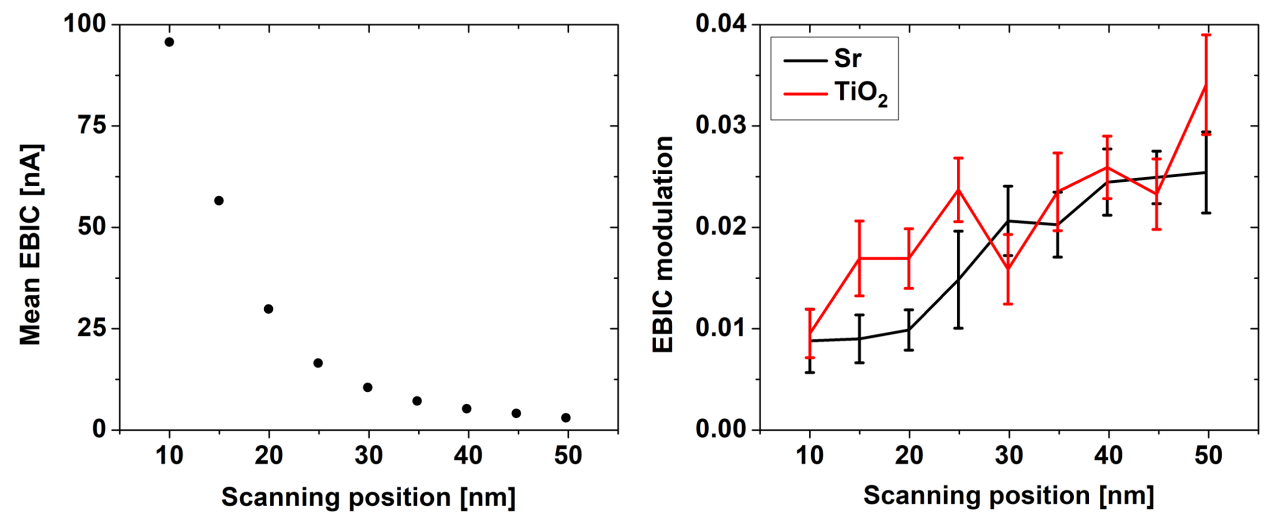

Figure 7.14: Mean EBIC of the PCMO-STNO lamella (left) as well as the modulation as defined in Eq. (7.5) for $\mathrm{Sr}$ as well as $\mathrm{TiO}_{2}$ columns (right) with respect to the distance to the interface. The EBIC modulation was extracted column-wise and subsequently averaged in scanning areas as the one shown in Fig. A.18 to eliminate effects caused by the distance to the interface. The scanning position represents the mean distance to the interface in the scanned area and the error bars are given by the standard deviation of the column-wise EBIC modulation.

facts. However, some preliminary ideas about possibly underlying mechanisms and strategies for future experiments shall be given. Assuming for a while that the additional EBIC signal is mainly caused by a cascade due to core-hole events leading to plasmon excitations [171, 172], which eventually decay to electron-hole-pairs or emit secondary electrons, the related mechanism could depend on the local concentration of free charge carriers. Obviously, a change of the latter is expected in the SCR, but strain might also be an influencing factor [173]. In fact, the strongest changes of the modulation on Sr columns appears at scanning position well outside the expected SCR (following Eq. (2.18)). Either way, if the spatial evolution is related to non-radiative decay channels of core-hole excitations, a correlation with the complementary radiative signal, e.g. obtained by coincidence microscopy [174], could lead to a more complete picture. Furthermore, a correlation with EELS data with a higher energy resolution, i.e. monochromation, is desirable to gain information about the previously mentioned low-energy excitation. 


\section{Chapter 8}

\section{Summarising Discussion and Outlook}

Finally, the results presented and discussed in the previous chapters shall be summarised and an outlook on how future studies building on them could be designed will be given.

\subsection{Method Development}

A significant part of this thesis is related to the implementation and development of transmission electron microscopy (TEM) methods, which have already been applied to a large extend in chapter 6 and 7 . However, the key advances shall be briefly summarised separately as they open up opportunities in other fields as well:

- Firstly, planview as well as in-situ biasing sample preparation strategies were presented. In the latter context a novel geometry with adjacent instead of opposed contacts was demonstrated, which enables easy wedge-shaped thinning and is expected to be well-suited for offaxis electron holography experiments [146]. Moreover, the planview preparation allows for straight-forward extraction of pristine surfaces, which could be of particular interest when studying e.g. electrochemical processes in gaseous or liquid environments [25].

- Secondly, a comprehensive software tool for 4D-STEM data acquisition and analysis was developed allowing for strain, electric and magnetic field mapping as well as fluctuation electron microscopy analysis 
and the creation of virtual detectors. Compared with accessible software solutions [107-109], the integration into DigitalMicrograph offering scripting commands to control the microscope led to the so-far unique possibility of data live-processing. In fact, it is to be expected that the combined architecture of data acquisition and analysis will facilitate future experiments even further as it allows for rather flexible adjustments to specific experimental needs and automation being of particular interest in in-situ experiments where the operator's time is scarce.

- Thirdly, strategies for STEM signal synchronisation and quantification were presented, which were mainly designed to increase the acquisition efficiency in electron beam induced current (EBIC) experiments. However, particularly the demonstrated approach of a posteriori synchronisation is applicable to a large variety of other signals as well including e.g. entire spectra, which typically cannot be fed to AD converters of scanning generators. In addition, the strategy could be easily extended to sparse sampling [133] being invaluable when investigating e.g. beam sensitive specimens.

\subsection{Phase Diagram of $\mathrm{Pr}_{1-x} \mathrm{Ca}_{x} \mathrm{MnO}_{3}$ (PCMO) Thin Films}

Both in-situ cooling as well as heating electron diffraction was performed on PCMO thin films to shine light on their phase diagram and draw comparisons to the bulk counterpart. Resulting highlights are:

- For $x=0.34$, the charge ordering parameter was successfully extracted by carefully disentangling the intensity of corresponding superstructure reflections from the main peak's background. This allowed for a precise determination of the critical temperature $T_{\mathrm{CO}}=218(4) \mathrm{K}$ in a $100 \mathrm{~nm}$ thick film being decreased by approximately $20 \mathrm{~K}$ in comparison with the bulk and highly consistent with complementary, indirect fingerprints such as an enhancement of the photovoltaic activity [17] resp. the lifetime of hot carriers [18].

- For $x=0.1$, the high-temperature structural transition known from bulk PCMO was found in a $400 \mathrm{~nm}$ thick film as well, however, with a slightly decreased critical temperature of approximately $600 \mathrm{~K}$ compared to roughly $675 \mathrm{~K}$ in the bulk [20]. In order to successfully ob- 
serve the accompanied changes of lattice parameters (by means of the previously developed 4D-STEM strain mapping) without strong hysteresis effects, an oxygen environment had to be employed. Importantly, the latter finding is likely not restricted to TEM investigations and hysteretic behaviour was reported in neutron diffraction studies as well $[19,51]$ intensifying the importance of the environment-controlled experiments.

- Additionally, for $x=0.1$, preliminary investigations at liquid nitrogen temperature were performed showing that by employing the developed planview preparation technique, i.e. after removing the substrate yielding a free-standing film, the in-plane diffraction spots of the alternating $a$ and $b$ twin structure can be separated and SAED is a promising candidate to resolve structural fingerprints of orbital ordering [28]. Furthermore, a strategy to observe a possible change in the extinction rules due to the latter by simply exciting systematic rows was suggested and should be included in future experiments.

- Lastly, even though not specifically related to thin films, a geometrical model was developed to describe Pbnm lattice parameters in the case of untwisted octahedra with a new parametrisation compared with existing models [50]. Applied to refined data obtained on bulk materials, it was shown that only few parameters are needed besides the lattice constants to describe the experimental findings accurately. In fact, this might pave the road for electron diffraction based refinements, possibly also transferable to thin films, and future studies should include the search for characteristic fingerprints of $d_{0}$ and $q_{2}$ e.g. first in simulated DPs. This appears particularly interesting as the remaining parameters, i.e. the lattice constants, can be observed with high-precision on the nanoscale due to the fabricated bullseye aperture presented in chapter 4, which was inspired by [128].

\subsection{Scanning Transmission Electron Beam Induced Current (STEBIC)}

Building on the achievements of P. Peretzki [29], the electronic setup, the synchronous data acquisition, as well as the in-situ biasing sample preparation was further optimised yielding a high output of STEM-EBIC results with the following achieved milestones: 
- Employing the one diode model, the bulk-like electronic behaviour of TEM lamellas could be disentangled from preparation induced serial and parallel resistances allowing for correction of differences between the experimentally measured and the actual short-circuit current and forming a quantitative bridge between macroscopic and nanoscale properties. Furthermore, in the best case, lamella with virtually identical current voltage characteristics to their bulk counterparts can be achieved.

- Qualitatively different excess charge carrier recombination activities of misfit dislocations depending on the core location in the SCR of PCMO-STNO interfaces were demonstrated. Importantly, subsequent developments such as strain and electric field mapping could reveal valuable information about underlying recombination mechanisms [69] and should be correlated to EBIC in future studies. In combination with the other presented advances in EBIC modelling, this might enable a quantitative description.

- The bulk diffusion length $L=10.3(2) \mathrm{nm}$, dead layer thickness $t_{\mathrm{d}}=$ $15(1) \mathrm{nm}$, and reduced surface recombination velocity $s=0.08(2) \mathrm{nm}^{-1}$ of STNO $(y=0.01)$ was quantified successfully showing a decrease of the former two in comparison with a five times lower doping concentration [29] (while the latter was not determined). Please note that also a lower acceleration voltage of $5 \mathrm{kV}$ instead of $30 \mathrm{kV}$ during final thinning was used herein. Furthermore, a qualitatively larger extension of the EBIC profiles in RP-PCMO compared with PCMO was demonstrated, which is consistent with an enhanced photovoltaic effect of the former at room temperature [27]. In a future experiment, STEM-EBIC on RP-PCMO should be combined with in-situ heating in order to check if this increased extension is related to charge ordering. In addition, it was pointed out that the EBIC profile in neither RP-PCMO nor PCMO can be modelled accurately by an exponential function, which might be related to heterogeneities of the thin films towards the interface and should be subject of further investigations.

- In-situ biased STEM-EBIC was performed on PCMO-STNO suggesting an avalanche effect for reverse-bias voltages below $-0.4 \mathrm{~V}$ and showing that the profile expands monotonously with the applied reversebias. In order to assess whether the junction in question can be described for instance by the box approximation, i.e. Eq. (2.18), further analytical and numerical modelling is necessary. However, an 
avalanche effect at the given field strength on the order of $\mathrm{MV} / \mathrm{cm}$ appears realistic and the presented data set might guide this modelling process and facilitate, e.g. in correlation with electric field mapping, a successful handshake of recombination descriptions in- and outisde the SCR. Furthermore, alternating external voltages to study dynamic effects should be included in future experiments.

- In a joint study including numerical modelling of EBIC and boron diffusion performed by D. A. Ehrlich and P. Pichler, a sub-0.1 ppm isoconcentration line of boron in a textured silicon solar cell was mapped on the nanoscale. Noteworthy, such low concentrations in heterogeneous systems are well beyond the theoretical detection limit of secondary ion mass spectrometry [170]. Furthermore, the two-dimensional effective recombination model used in the EBIC simulation facilitated the calculation of entire signal maps for the first time. Still, deviations in the SCR are observed, which appears natural as the effective recombination parameters were adjusted in the neutral regions. However, enabled by the presented experimental advances, these deviations, possibly in combination with the knowledge gained by the in-situ biased data mentioned above, should help finding more sophisticated models to close the gap between experiment and simulation. In more detail, a reasonable next step would be the inclusion of surface charges as well as reemission from the SRH-type recombination centers.

- Lastly, the existence of atomic modulations of EBIC sigals was demonstrated in STNO, PCMO and Si. In addition, a correlation between core-loss events and the additional EBIC was revealed by synchronously acquired EEL spectra and the evolution of the modulation in STNO towards the rectifying interface with PCMO was analyzed. An analytical model explaining the modulation quantitatively is yet to be found, however, preliminary ideas about the underlying mechanisms and future experimental strategies were suggested. 
138 CHAPTER 8. SUMMARISING DISCUSSION AND OUTLOOK 


\section{Appendix A}

\section{A.1 Rotation Matrix Convention}

$$
\mathbf{R}(\vec{n}, \theta)=\left(\begin{array}{ccc}
n_{1}^{2}(1-\cos \theta)+\cos \theta & n_{1} n_{2}(1-\cos \theta)-n_{3} \sin \theta & n_{1} n_{3}(1-\cos \theta)+n_{2} \sin \theta \\
n_{2} n_{1}(1-\cos \theta)+n_{3} \sin \theta & n_{2}^{2}(1-\cos \theta)+\cos \theta & n_{2} n_{3}(1-\cos \theta)-n_{1} \sin \theta \\
n_{3} n_{1}(1-\cos \theta)-n_{2} \sin \theta & n_{3} n_{2}(1-\cos \theta)+n_{1} \sin \theta & n_{3}^{2}(1-\cos \theta)+\cos \theta
\end{array}\right)
$$

\section{A.2 Uniqueness of the Pbnm Rotation Axis}

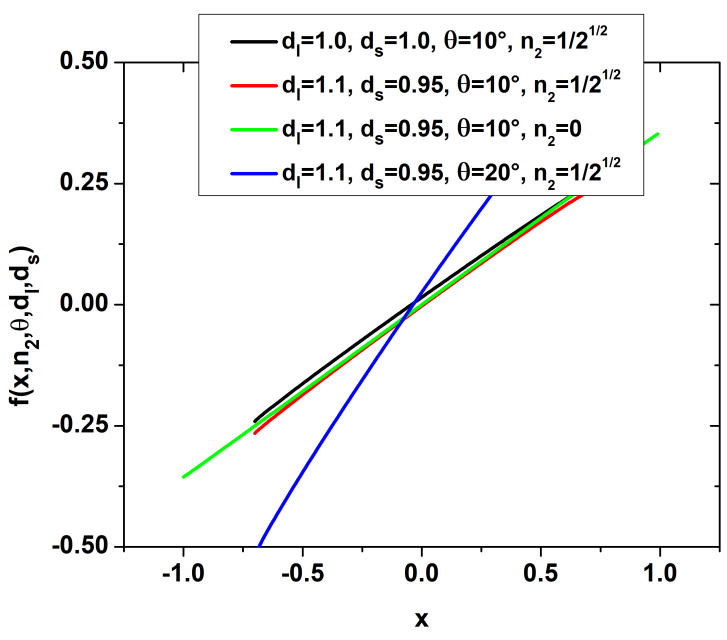

Figure A.1: Values of $f\left(x, n_{2}, \theta, d_{\mathrm{l}}, d_{\mathrm{s}}\right)$ as defined in Eq. (2.8) showing that a unique value for $n_{1}$ exists over a wide range of choices for the remaining parameters. 


\section{A.3 4D-STEM Analysis: Virtual Masking}

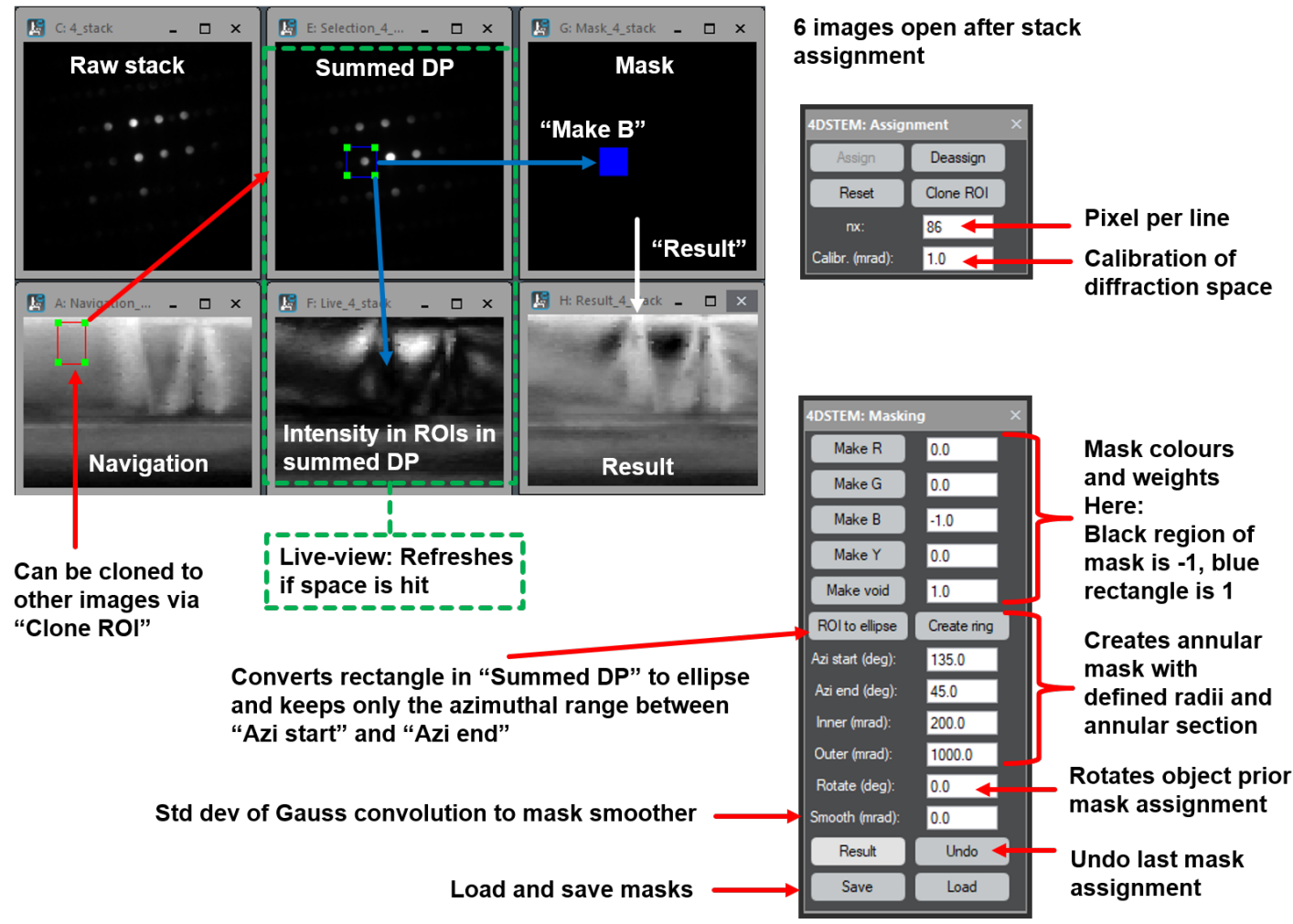

Figure A.2: Workflow of the assignment and masking interface of the 4DSTEM analysis tool. 


\section{A.4 4D-STEM Analysis: Bragg Analysis}

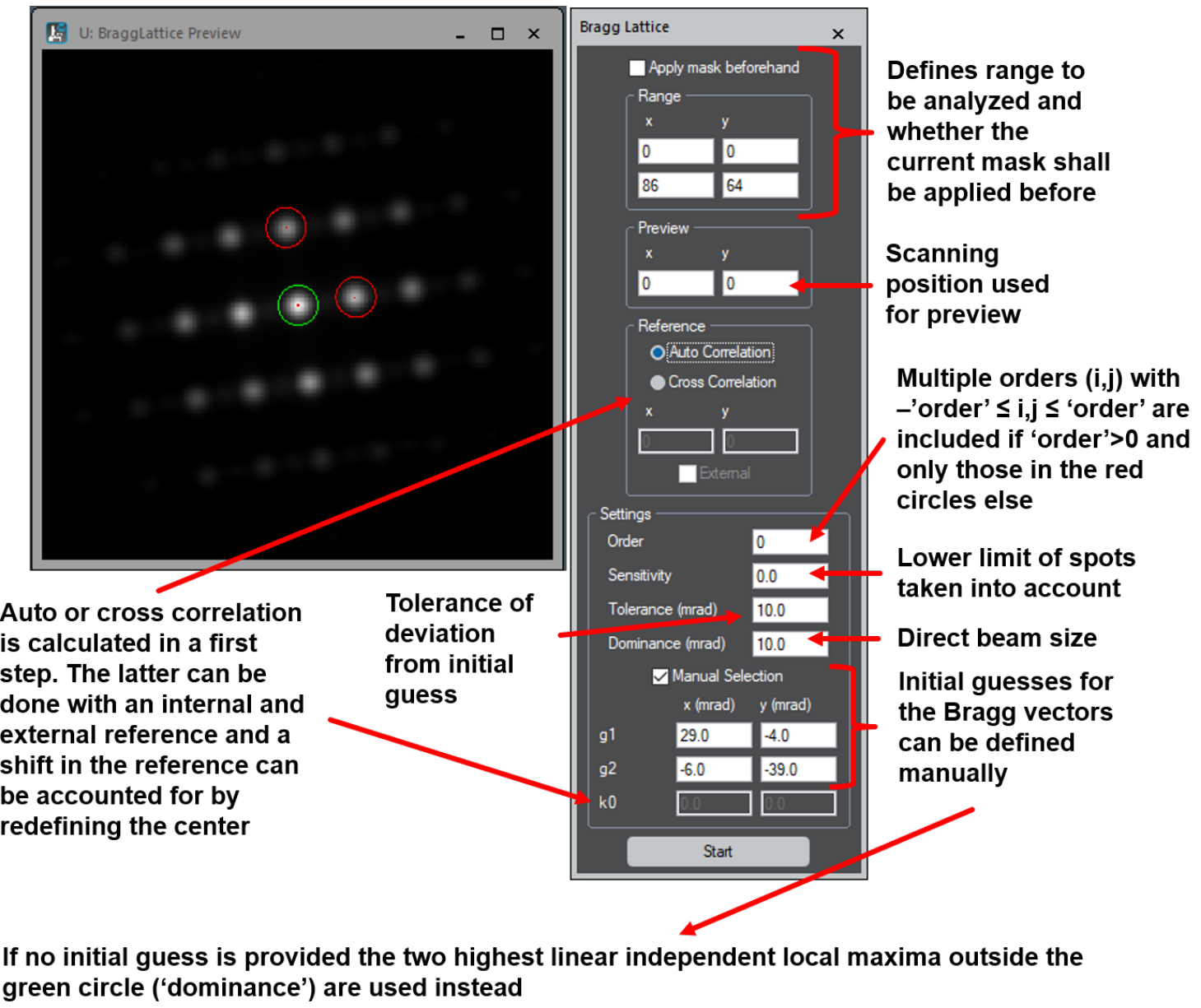

Figure A.3: Workflow of the Bragg analysis interface of the 4D-STEM analysis tool. 


\section{A.5 4D-STEM Analysis: First Moment}

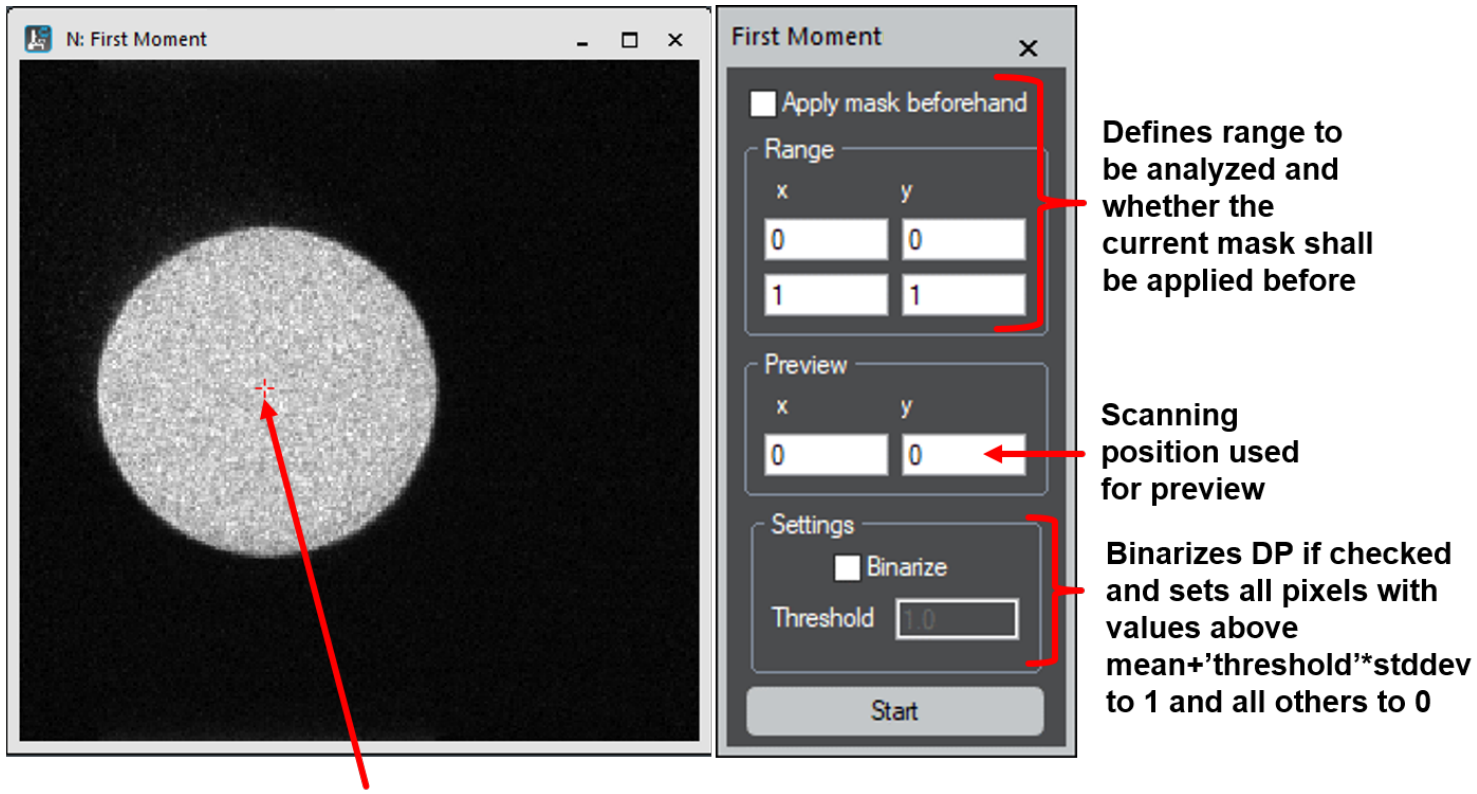

Live view of extracted first moment in preview

Figure A.4: Workflow of the first moment interface of the 4D-STEM analysis tool. 


\section{A.6 4D-STEM Analysis: Virtual DPC}

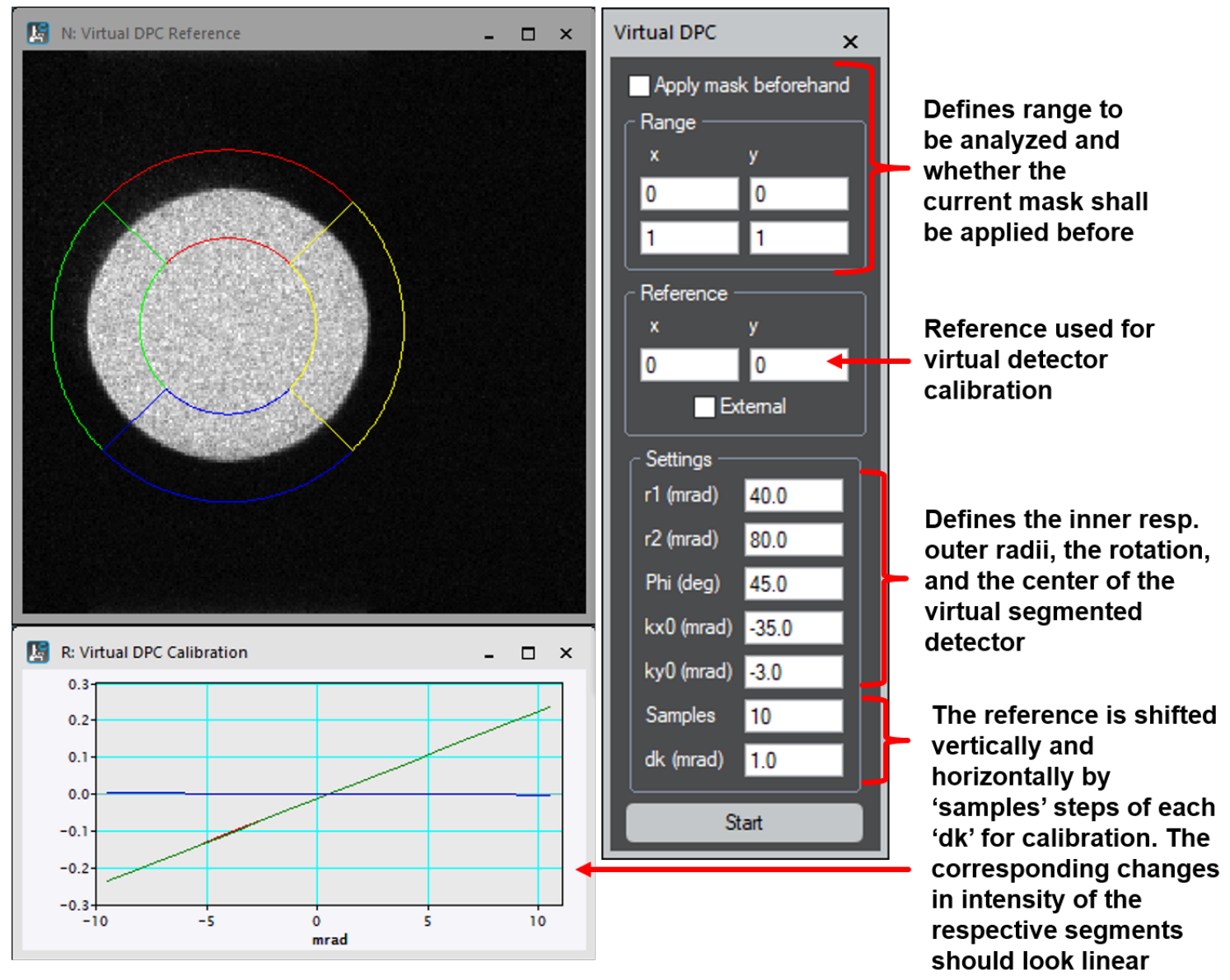

Figure A.5: Workflow of the virtual DPC interface of the 4D-STEM analysis tool. 


\section{A.7 4D-STEM Analysis: Edge Detection}

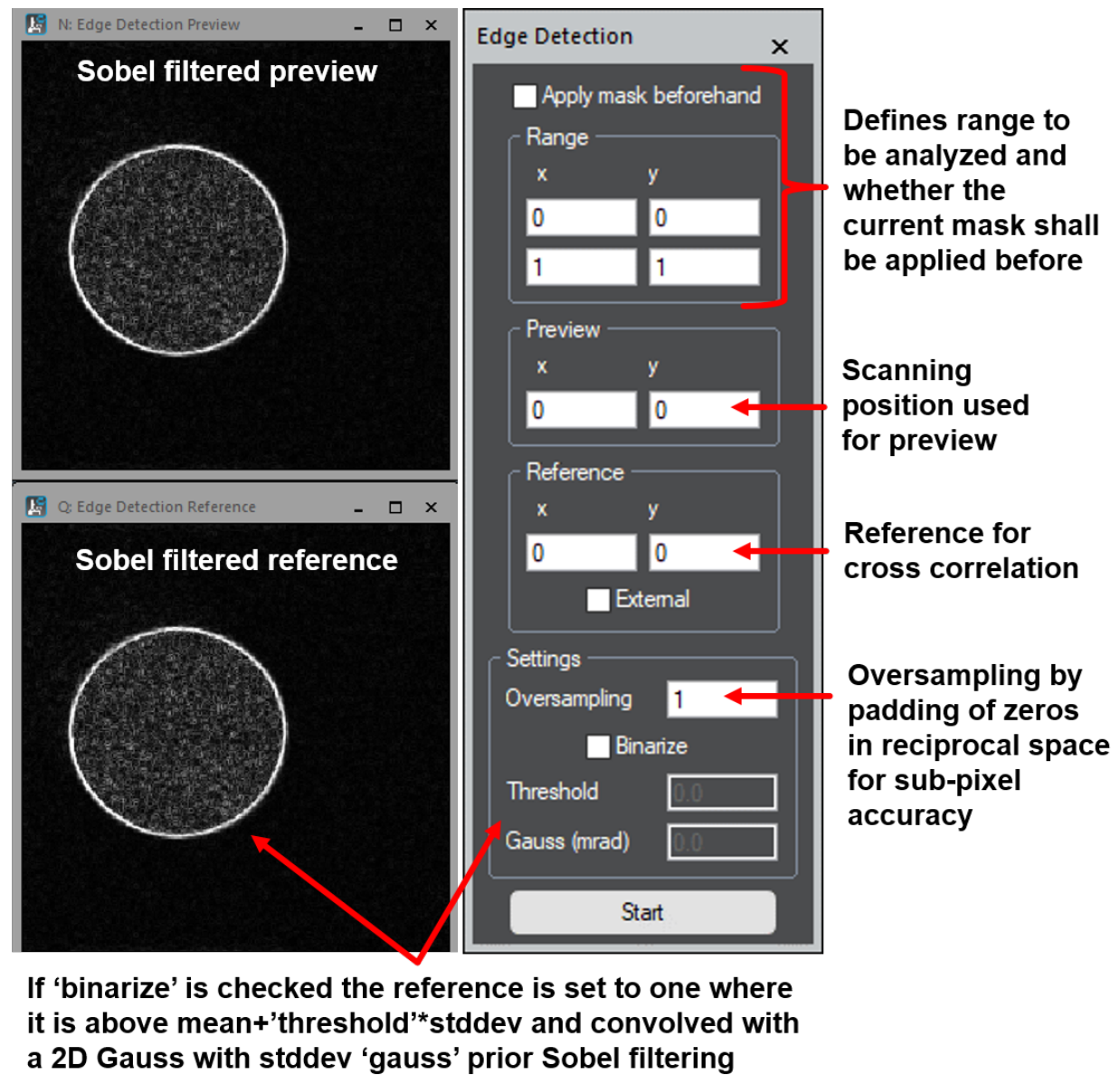

Figure A.6: Workflow of the edge detection interface of the 4D-STEM analysis tool. 


\section{A.8 4D-STEM Analysis: FEM}

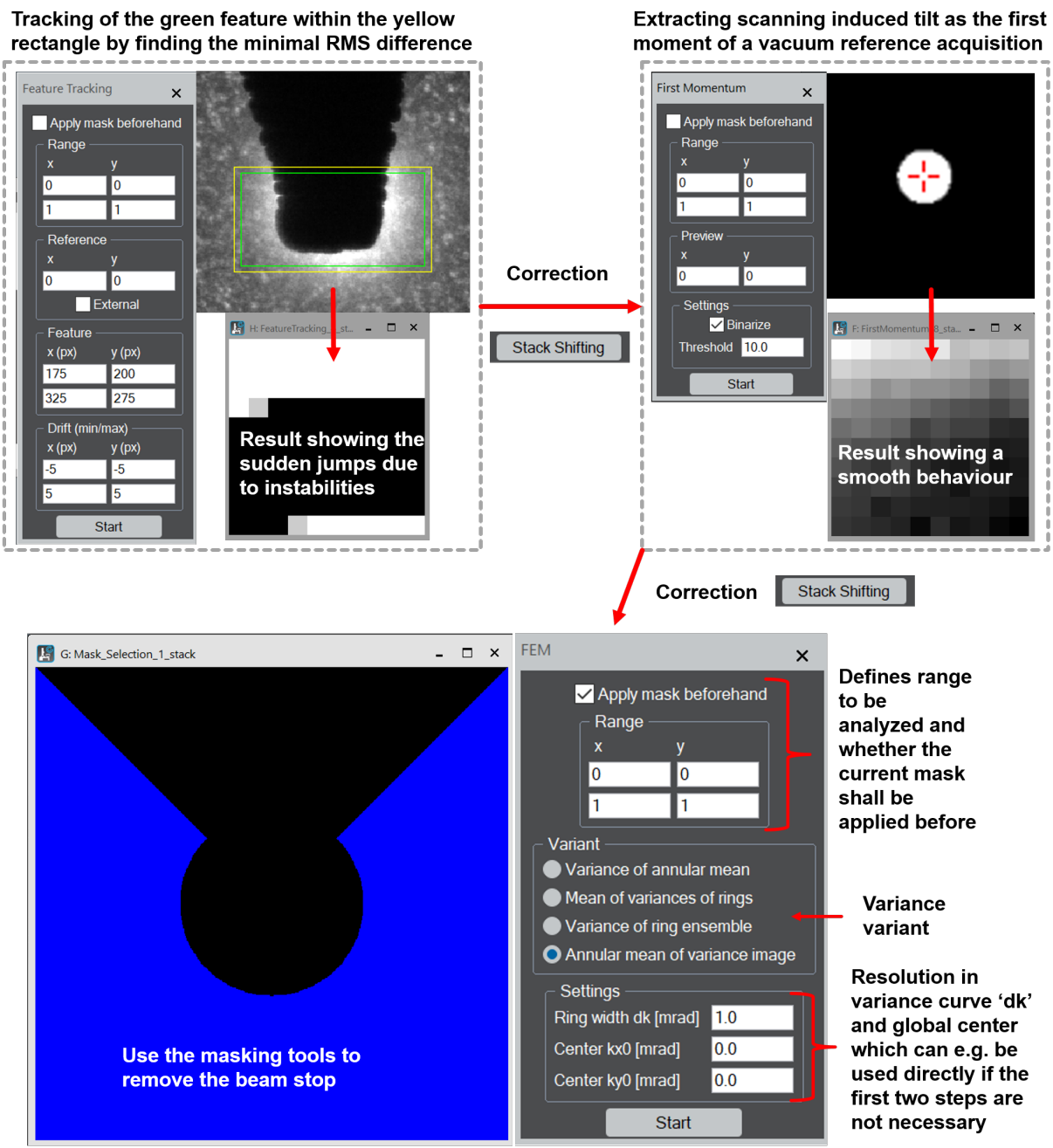

Figure A.7: Workflow of FEM data processing by combining several functions of the 4D-STEM analysis tool. 


\section{A.9 ADC Calibration}
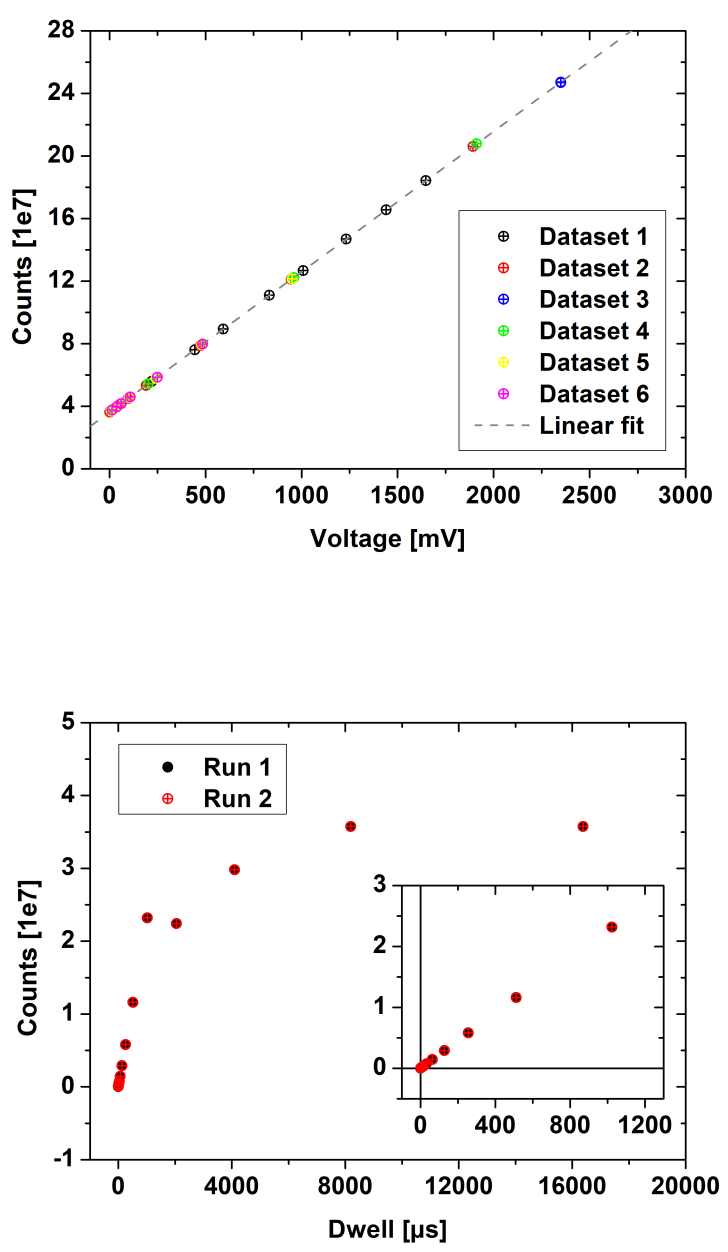

Figure A.8: Voltage dependence of the count output of the ADC integrated in the Gatan DigiScanII unit for a fixed dwell time of $30 \mathrm{~ms}$. Please note that the calibration is only valid if hardwaresynchronisation is used in the DigiScanII settings.

Figure A.9: Dwell time dependence of the count output of the ADC integrated in the Gatan DigiScanII unit for an input voltage of $0 \mathrm{~V}$. Only $30 \mathrm{~ms}$ dwell times have been used for quantitative data collection in this thesis. 


\section{A.10 A posteriori synchronization of scanning trans- mission electron microscopy signals with kilo- pixel per second acquisition rates (Supple- mentary Material)}

The following two source codes were published as supplementary material in [160] and are reprinted with permission.

\section{Acquisition}

// Script for data acquisition designed for a posteriori reconstruction from a continuously acquired signal with a distinct marker signal before and behind every scanning line:

// The user must specify the desired dwell time and horizontal and vertical scanning points in the contructed user interface

// The scanning region has to be specified by a rectangular ROI in a DigiScan reference frontimage whereas the marker position

// has to be given by point ROI (the beam positioning tool of DigiScan is recommended since it is non-volatile).

// Date: 27th February 2019

// Author: Tobias Meyer, University of Goettingen

// Mail: tmeyer@uni-goettingen.de

// The architecture of the user interface is based closely on the script 'Hollow_Cone_Scanning.s' by D. R. G. Mitchell, J. Olivier (NMMU, South Africa) and J. O' Connell (NMMU, South Africa) (Version:20111205)

// Internal variables number dwell, nx, ny, sx, sy, dxx, dxy, dyx, dyy, roi_t, roi_l, roi_b, roi_r, marker_x, marker_y, ScanIsOn, Aposteriori_UI_ID

// User interface class

Class Aposteriori_UI : UIFrame

\{

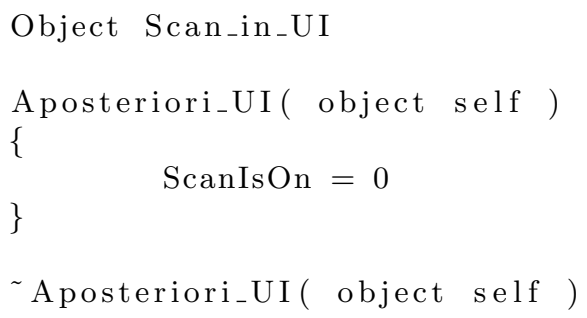




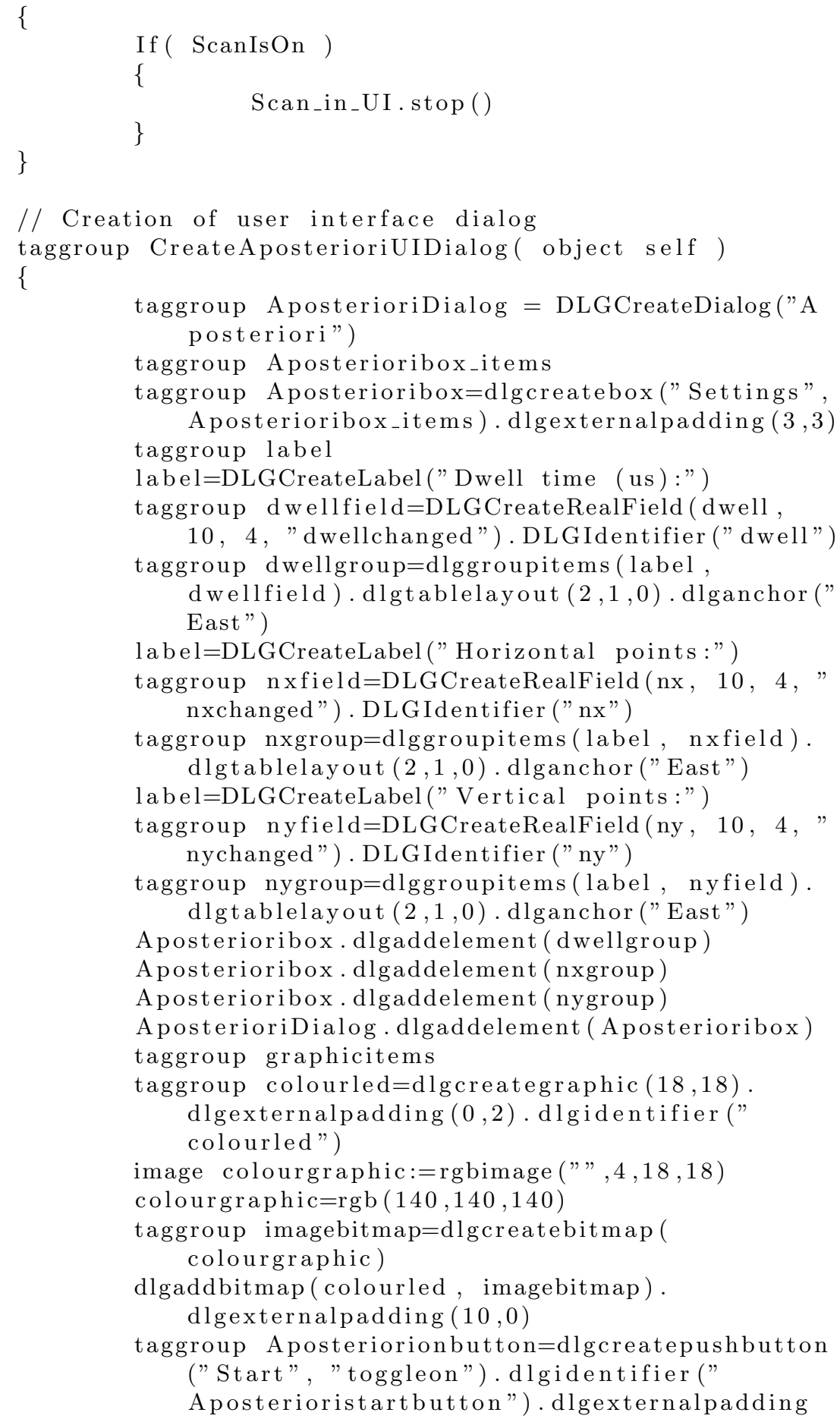




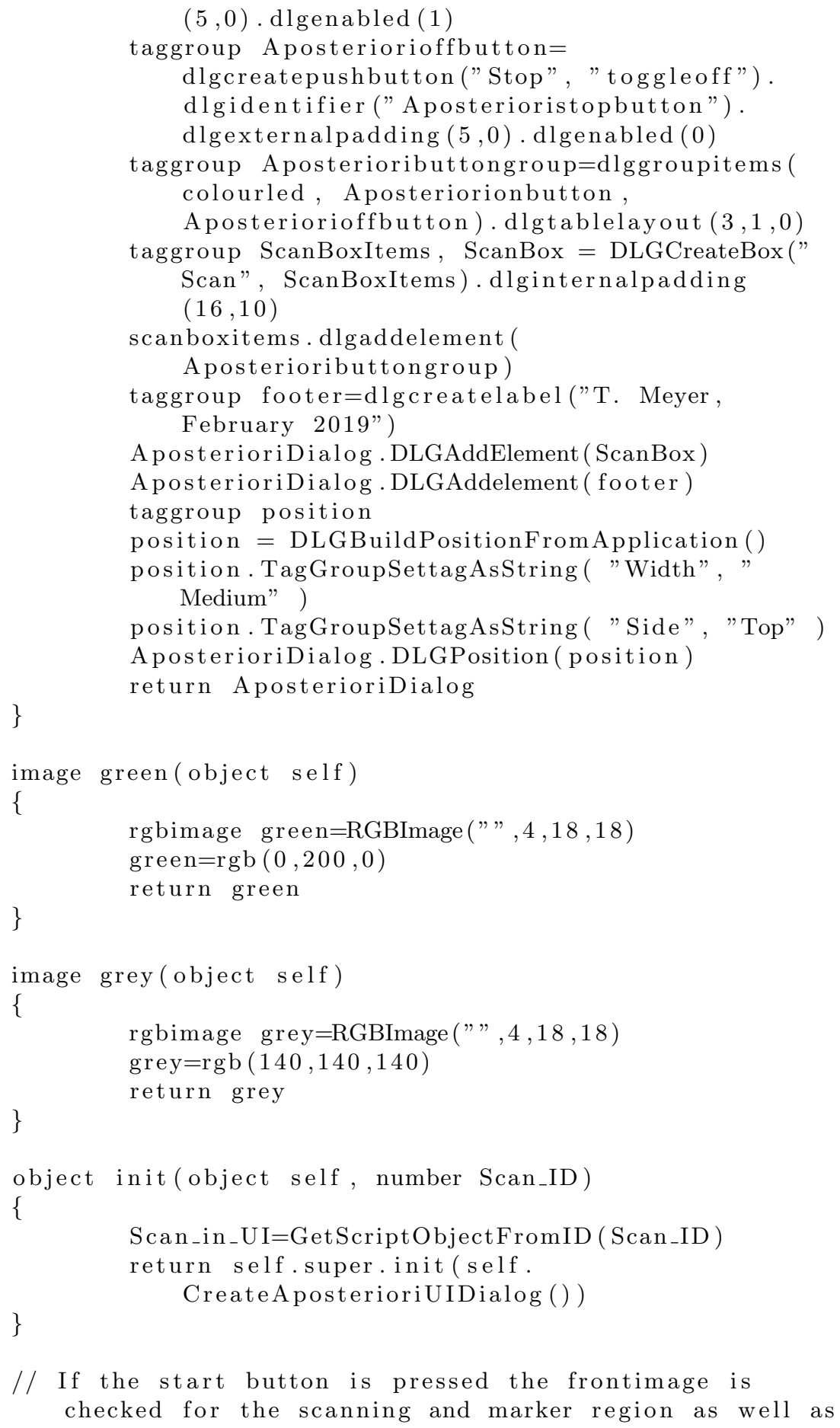


the internal DigiScan pixel steps .

// Subsequently, after all necessary variables are saved internally, user interface actions are disabled, scan control is enforced, and the scanning thread gets evoked.

void toggleon (object self) \{

image dsref:=getfrontimage ()

if (! DSIsValidDSImage (dsref)) throw("A DigiScan reference image is needed!") // Check whether the frontimage is a DigiScan reference image

// Check if the desired ROIs are defined in the reference image and get the scanning range as well as the marker position

if ( imagedisplaycountrois (imagegetimagedisplay ( dsref , 0$)$ ( ) $<$ ) throw ("A rectangular scanning ROI and a point ROI as marker position are needed!")

if ( ! ( ImageDisplayGetROI ( imagegetimagedisplay ( dsref ,0),0). ROIIsPoint () \&\&

ImageDisplayGetROI ( imagegetimagedisplay (dsref $, 0), 1)$. ROIIsRectangle ()) || (

ImageDisplayGetROI ( imagegetimagedisplay (dsref $, 0), 1)$. ROIIsPoint () \&\& ImageDisplayGetROI( imagegetimagedisplay ( dsref, 0$), 0)$.

ROIIsRectangle()))) throw ("A rectangular scanning ROI and a point ROI as marker position are needed!")

if ( ImageDisplayGetROI ( imagegetimagedisplay (dsref $, 0), 0)$. ROIIsPoint ( ) $)\{$ ImageDisplayGetROI ( imagegetimagedisplay ( dsref, 0$), 0$ ). ROIGetPoint ( marker_x , marker_y ); ImageDisplayGetROI ( imagegetimagedisplay ( dsref, 0$), 1)$. ROIGetRectangle (roi_t, roi_l, roi_b, roi_r );

else \{ ImageDisplayGetROI (imagegetimagedisplay ( dsref, 0$), 1)$. ROIGetPoint ( marker_x , marker_y ); ImageDisplayGetROI ( imagegetimagedisplay ( dsref $, 0), 0)$. ROIGetRectangle (roi_t, roi_l, roi_b, roi_r );\}

// Get the image size as well as the DigiScan pixel steps

getsize (dsref, sx, sy)

ImageGetTaggroup (dsref) . tagGroupGettag AsDouble (" DigiScan: Horizontal Pixel Step",dxx)

ImageGetTaggroup (dsref) .tagGroupGettag AsDouble (" DigiScan:Horizontal Perpendicular Pixel Step $"$, dxy )

ImageGetTaggroup (dsref) . tagGroupGettag AsDouble (" DigiScan: Vertical Pixel Step",dyx) 


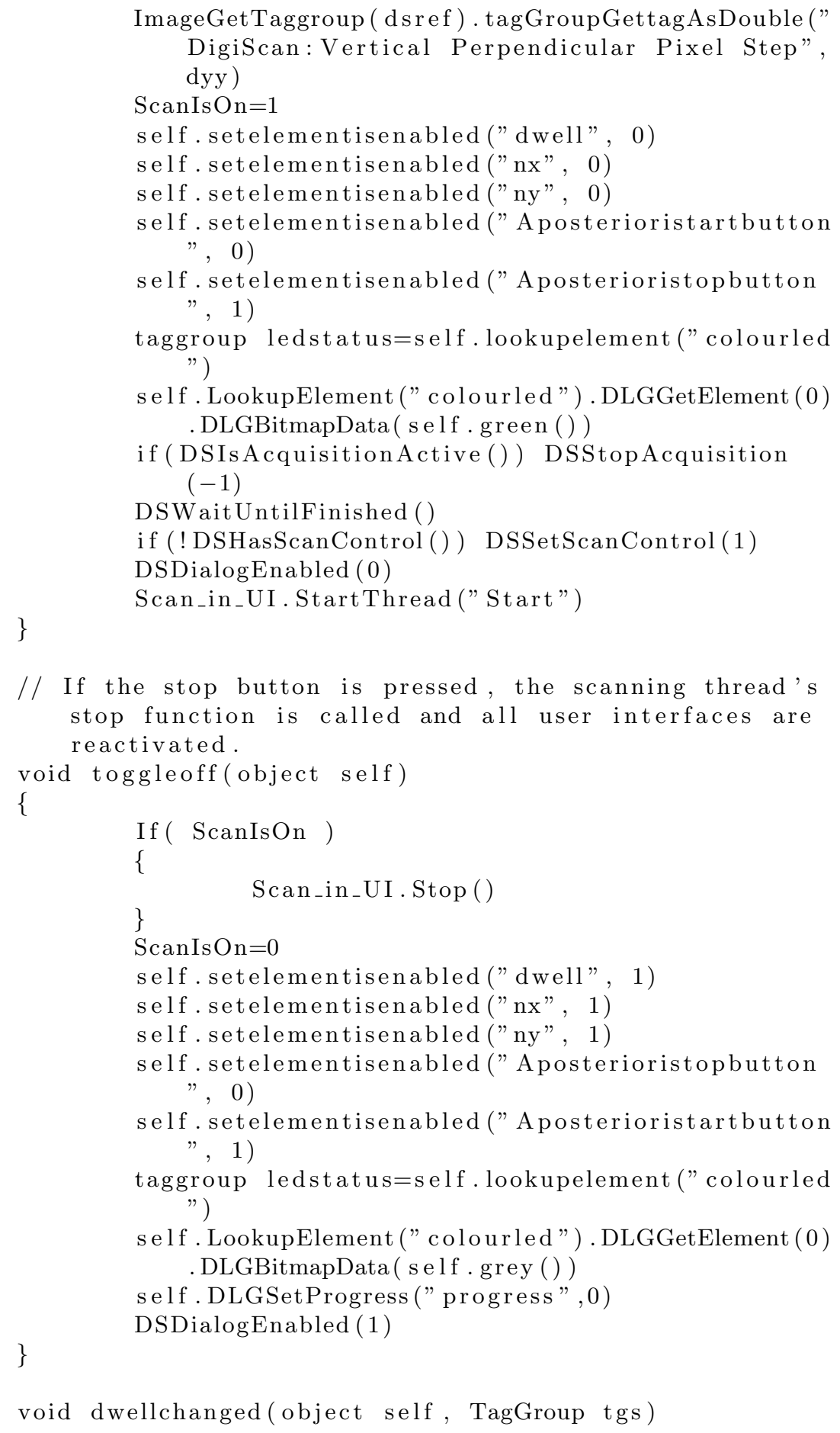




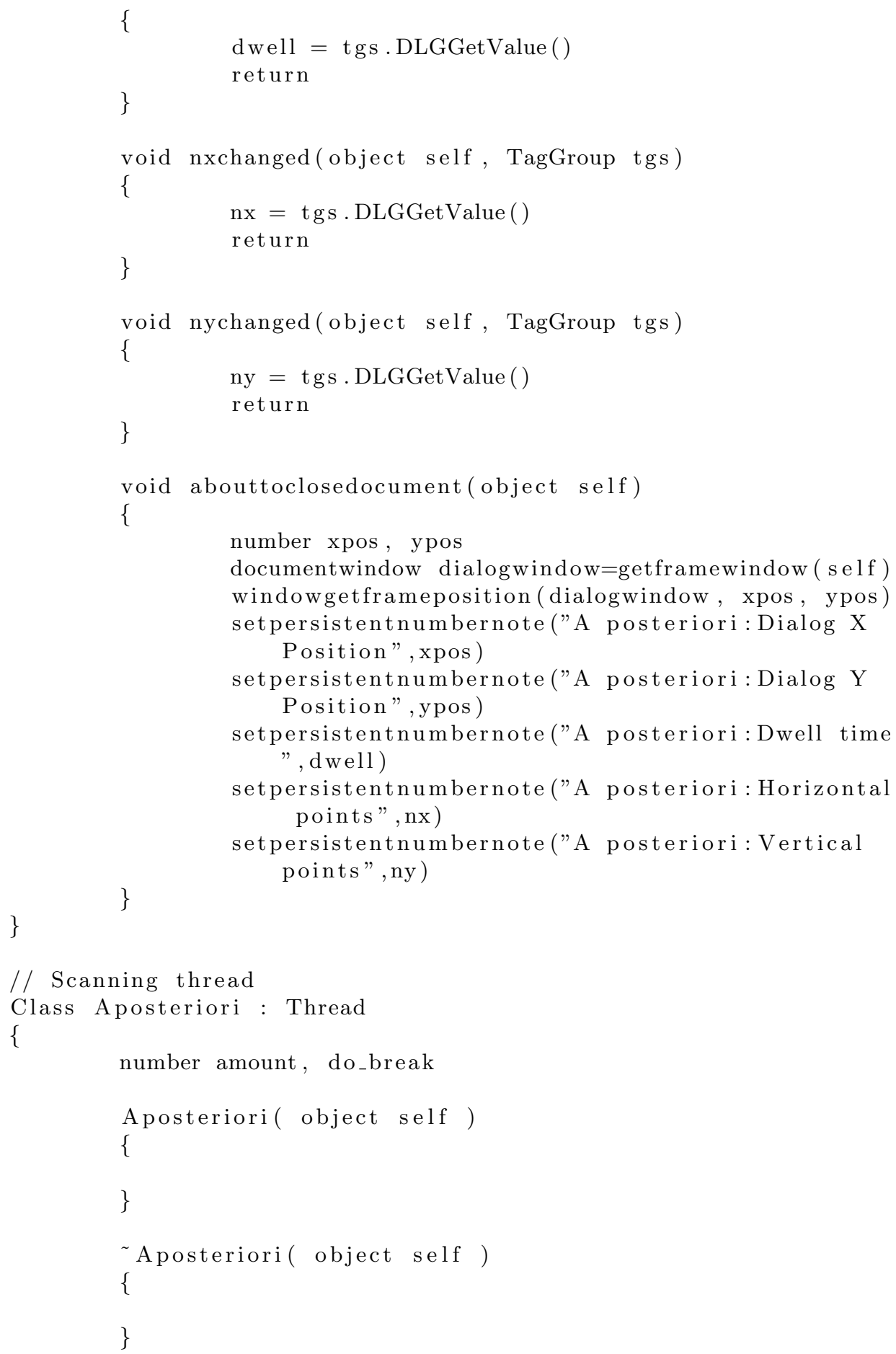




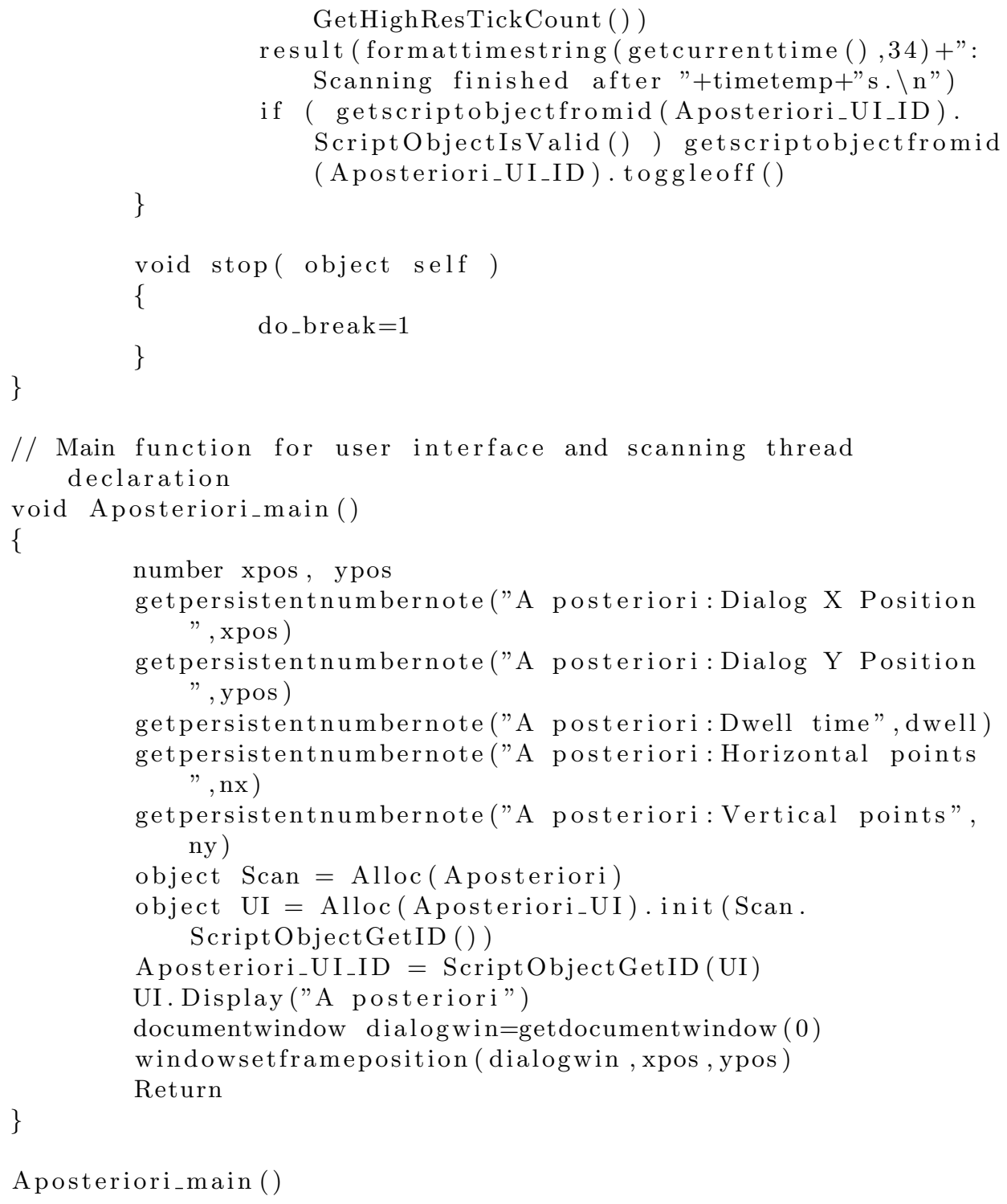

\section{Analysis}

// Script for a posteriori data reconstruction from a continuous signal acquisition with a distinct marker signal before and behind every scanning line:

// The user must specify the amount of horizontal and vertical scanning points as well as the starting index, i.e. the position of the first marker signal.

// Furthermore, the threshold between marker signal and scanning signal has to be specified as well as whether the marker 
signal is greater or lower than the threshold.

// After the reconstruction the user is asked if the indices of the beginnings and endings of lines shall be given as output images as well, which can be used

// for data reconstruction of different signals from the same acquisition, e.g. in the case of EELS the beginnings and endings can be determined by the total

// sum of counts and subsequently applied to reconstruct coreloss data in which the marker signal might be harder to localize.

// Date: 27th February 2019

// Author: Tobias Meyer, University of Goettingen

// Mail: tmeyer@uni-goettingen.de

// Input

number nx, ny, start, threshold, greater

if (!getnumber ("Number of horizontal scanning points:",128,nx)) exit $(0)$

if (! getnumber("Number of vertical scanning points:",128, ny)) exit $(0)$

if (!getnumber("Starting index:",0, start)) exit (0)

if (! getnumber ("Threshold between signal and marker:", 1 , threshold ) exit $(0)$

if (! getnumber(" Marker signal lower/greater than threshold $(0 / 1)$ :",0, greater)) exit (0)

// Internal variables

image in:=getfrontimage(). imageclone (), out=realimage (" ", 4, nx , ny ), beginnings=realimage (" ", 4,ny), endings=beginnings

if $($ greater $)\{\operatorname{in} *=-1$; threshold $*=-1 ;\} / /$ Invert the signal if the marker signal is greater than the threshold

number $n=$ imagegetdimensionsize (in , 0$)$, line $=0$, marker $=1$

for (number $\mathrm{i}=$ start $; \mathrm{i}<\mathrm{n} \& \&$ line $<$ ny $; \mathrm{i}++$ )

\{

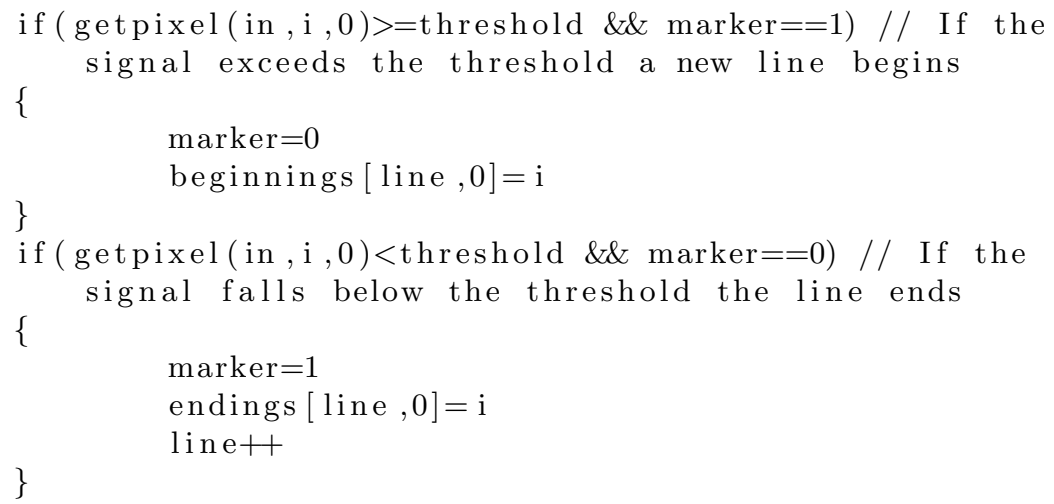




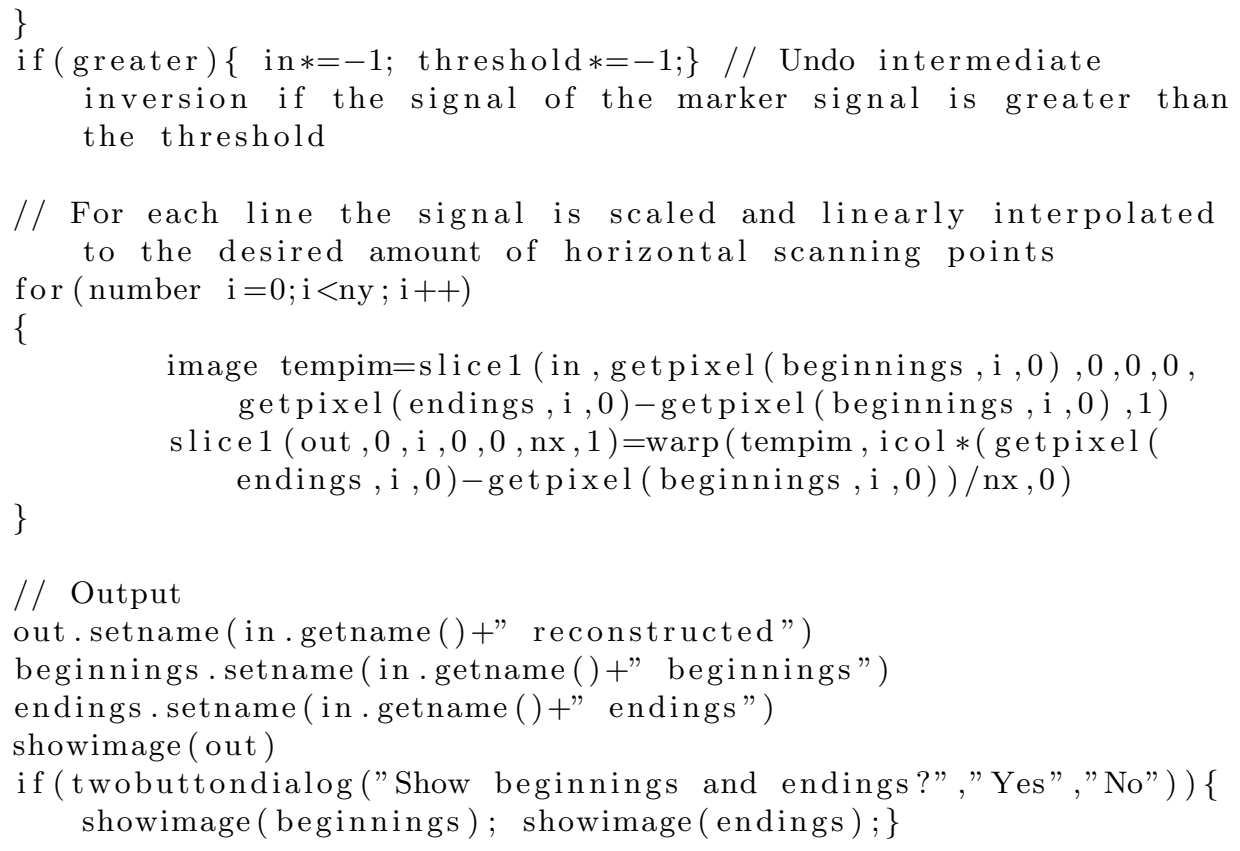

\section{Analysis of a second signal}

// Script for a posteriori data reconstruction with given beginnings and endings of lines from the script , Aposteriori_Analysis.s':

// The user must specify the amount of horizontal scanning points as well as the beginnings, endings and signal images.

// The script can be used for data reconstruction of a signal different from the one used to determine the beginnings and endings,

$/ /$ e.g. in the case of EELS the beginnings and endings can be determined by the total sum of counts and subsequently applied with this script

// to reconstruct core-loss data in which the marker signal might be harder to localize.

// Date: 27th February 2019

// Author: Tobias Meyer, University of Goettingen

// Mail: tmeyer@uni-goettingen.de

// Input

number $\mathrm{nx}$

image beginnings, endings, in

if (! getnumber("Number of horizontal scanning points:",128,nx)) exit $(0)$

if (! getthreelabeledimageswithprompt("Please specify the input images:" ," ," Beginnings", beginnings ," Endings", endings ," Signal 


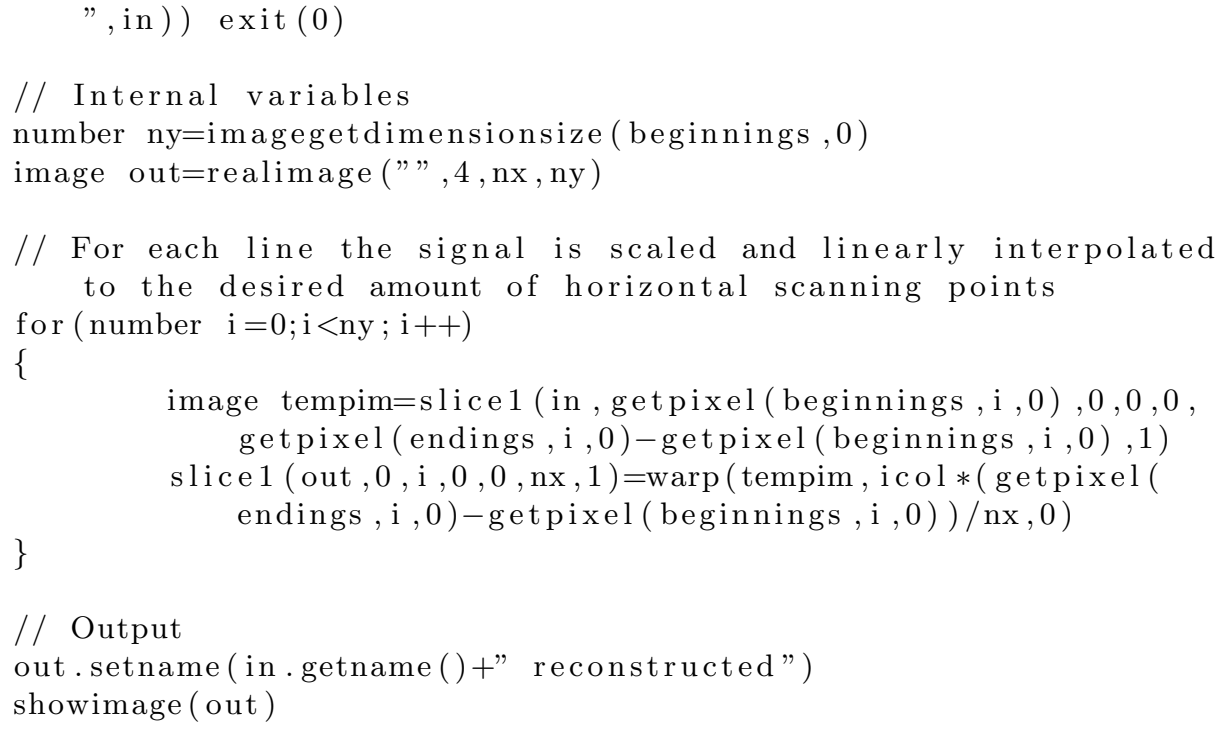

\section{A.11 Macroscopic Perovskite Thin Film Samples}

\begin{tabular}{|c|c|c|c|c|c|c|}
\hline Chap. / Sec. & Film & Substrate & Anneal. & Front cont. & Rear cont. & Label \\
\hline 6.1 & $\begin{array}{c}\text { PCMO } \\
x=0.34 \\
100 \mathrm{~nm}\end{array}$ & $\begin{array}{c}\text { STNO } \\
y=0\end{array}$ & $\begin{array}{c}20 \mathrm{~h} \\
900^{\circ} \mathrm{C}\end{array}$ & - & - & BK38 \\
\hline $\begin{array}{l}6.2 \\
6.3\end{array}$ & $\begin{array}{l}\text { PCMO } \\
x=0.1 \\
400 \mathrm{~nm}\end{array}$ & $\begin{array}{c}\text { STNO } \\
y=0\end{array}$ & $\begin{array}{c}20 \mathrm{~h} \\
900^{\circ} \mathrm{C}\end{array}$ & - & - & BK77 \\
\hline $\begin{array}{c}6.2 \\
\text { (XRD } \\
\text { only) }\end{array}$ & $\begin{array}{l}\text { PCMO } \\
x=0.1 \\
400 \mathrm{~nm}\end{array}$ & $\begin{array}{c}\text { STNO } \\
y=0\end{array}$ & $\begin{array}{l}20 \mathrm{~h} \\
900^{\circ}\end{array}$ & - & - & BK78 \\
\hline 7 & $\begin{array}{c}\text { PCMO } \\
x=0.34 \\
100 \mathrm{~nm}\end{array}$ & $\begin{array}{c}\text { STNO } \\
y=0.01\end{array}$ & $\begin{array}{l}20 \mathrm{~h} \\
900^{\circ}\end{array}$ & $\mathrm{Pt}$ & $\mathrm{Ti} / \mathrm{Au}$ & BK68 \\
\hline 7 & $\begin{array}{c}\text { RP-PCMO } \\
x=1.5 \\
100 \mathrm{~nm}\end{array}$ & $\begin{array}{c}\text { STNO } \\
y=0.01\end{array}$ & - & $\mathrm{Pt}$ & $\mathrm{Ti} / \mathrm{Au}$ & $\begin{array}{l}\text { RP-PCMO } \\
\text { Sputter110 }\end{array}$ \\
\hline
\end{tabular}

Table A.1: Overview of the macroscopic thin film samples used in this dissertation as well as relevant ion beam sputtering parameters. 


\section{A.12 Charge Ordering in PCMO $(x=0.34)$}

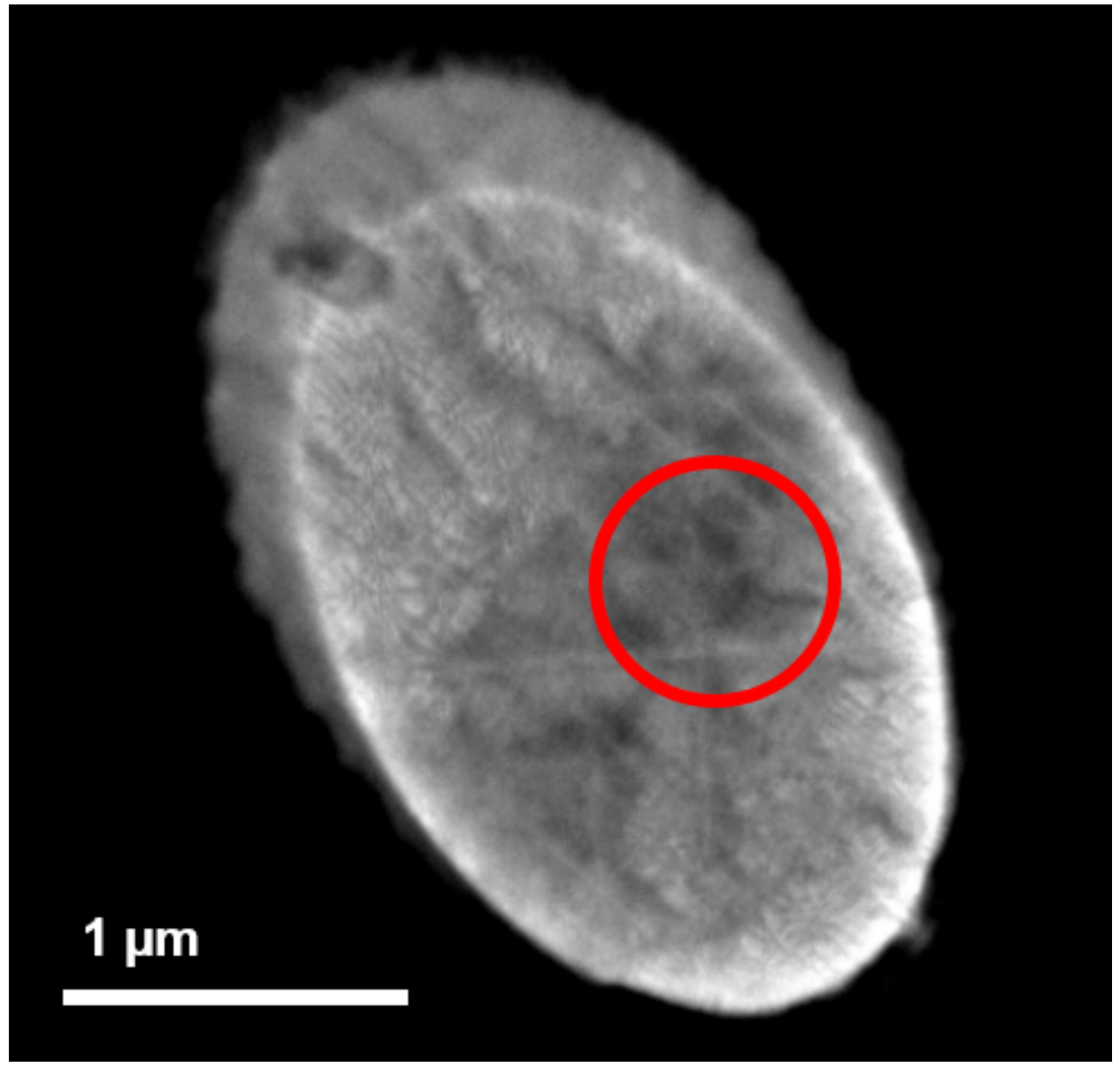

Figure A.10: TEM overview of the PCMO $(x=0.34)$-STO planview lamella mounted across a hole of the $\mathrm{Au}$ evaporated silicon nitride membrane. The red circle indicates the area investigated with SAED. 


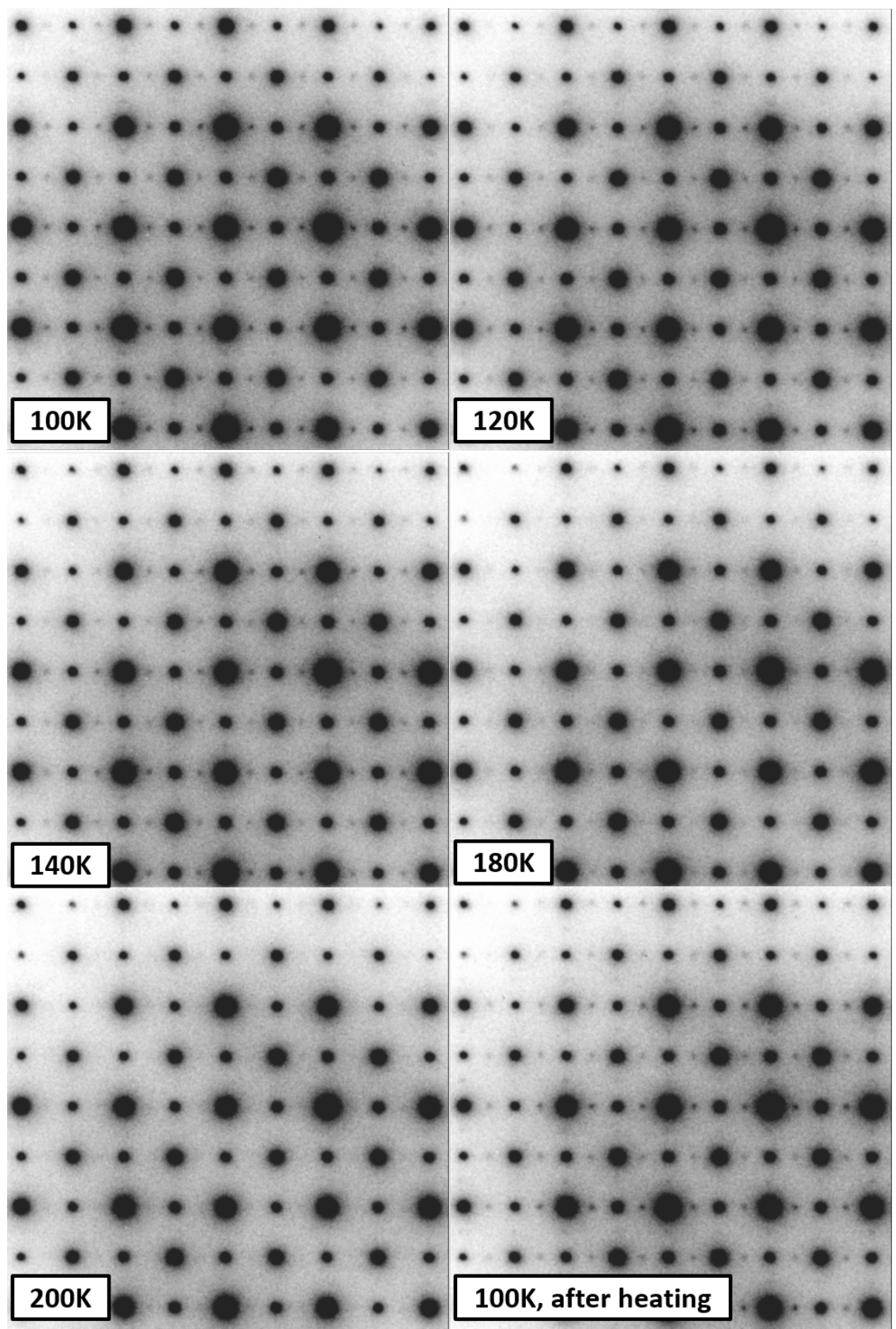

Figure A.11: Series of diffraction patterns collected while looping the temperature from $100 \mathrm{~K}$ to $200 \mathrm{~K}$ to $100 \mathrm{~K}$. The zone axis is slightly drifting in time, i.e. the intensity of spots in the upper left area gets weaker. 


\section{A.13 Domain Boundaries in PCMO $(x=0.1)$}

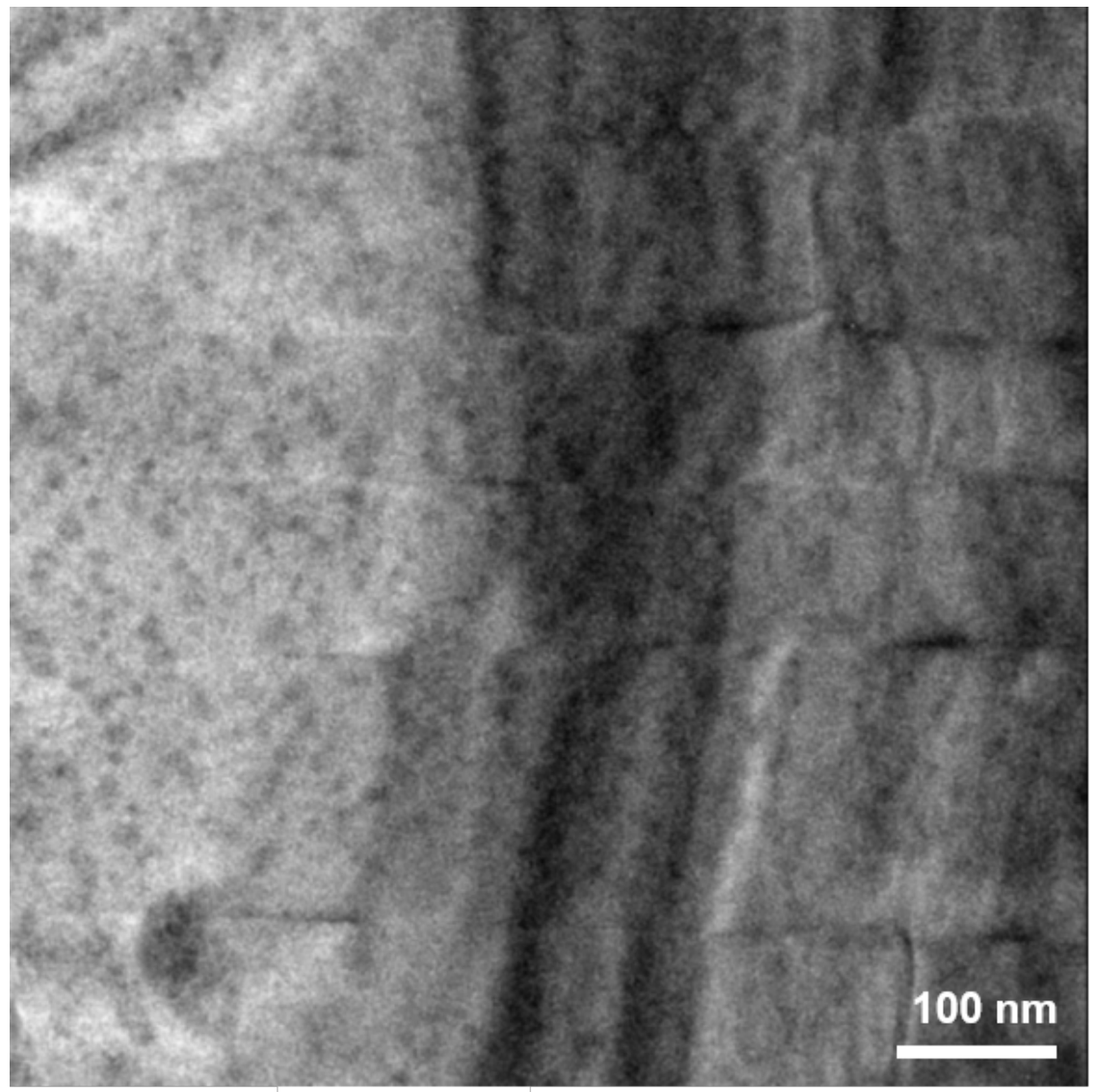

Figure A.12: Defocused TEM bright-field image of the alternating domain structure with horizontal boundaries indicated in the left part of Fig. 6.5. 


\section{A.14 Determination of Decay Lengths from EBIC Profiles}

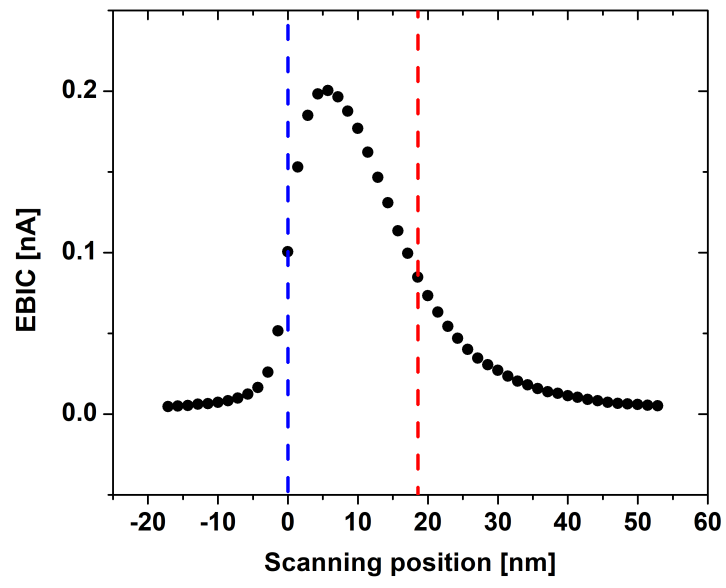

Figure A.13: Exemplary EBIC profile of a PCMO-STNO junction with the inner-most positions yielding the smallest standard deviation of the decay length when fitting an exponential function to the tails marked in blue resp. red for PCMO resp. STNO. 


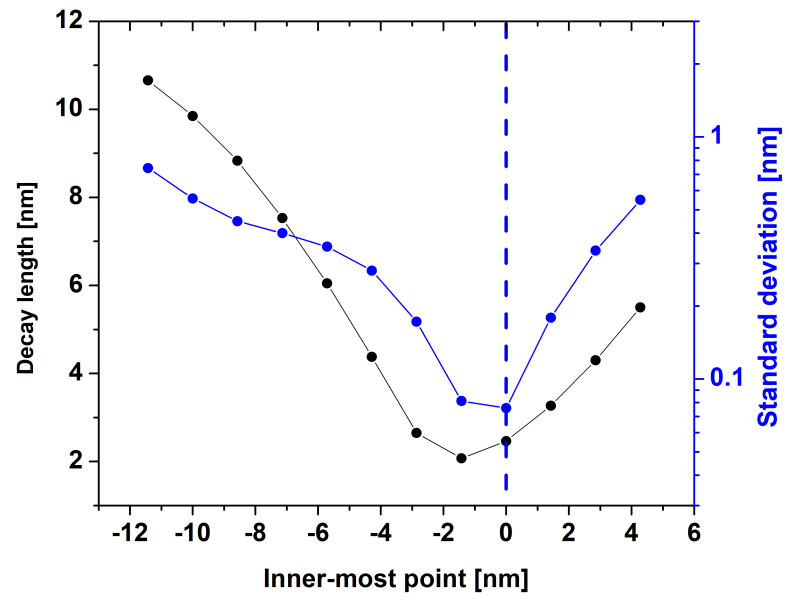

Figure A.14: Decay length (black) as well as its standard deviation (blue) when fitting an exponential function to the tail in PCMO (see Fig. A.13) with respect to the inner-most point of the fitting interval.

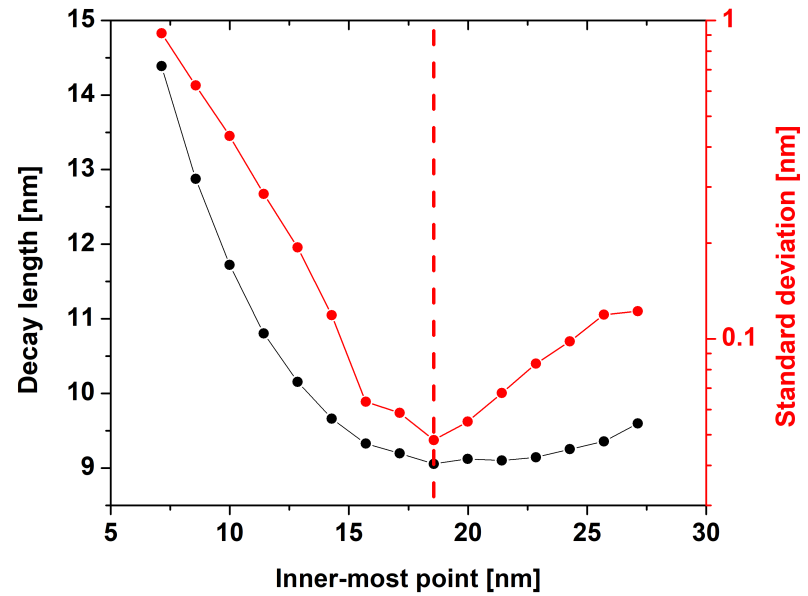

Figure A.15: Decay length (black) as well as its standard deviation (red) when fitting an exponential function to the tail in STNO (see Fig. A.13) with respect to the inner-most point of the fitting interval. 


\section{A.15 Interface Localisetion of PCMO-STNO Het- erojunctions}

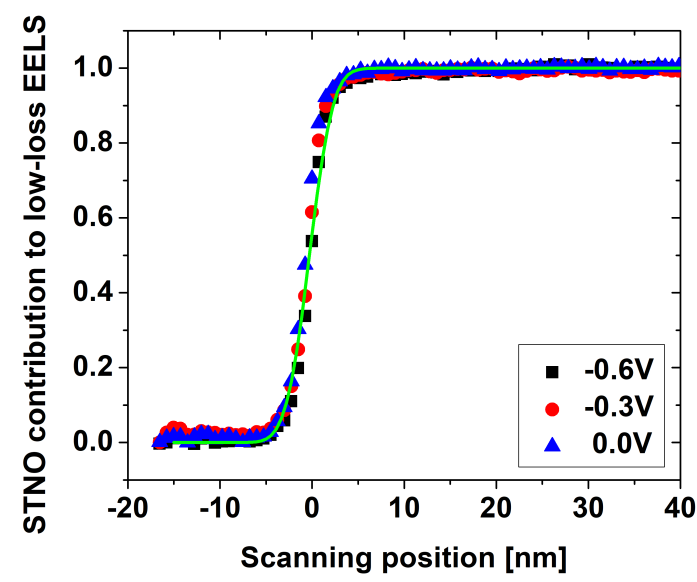

Figure A.16: STNO contribution to the EELS plasmon singal es defined in Eq. (7.3) with respect to the scanning positions for different reverse bias voltages. The intersection with 0.5 is defined as the interface position. The green line corresponds to an error function with a standard deviation of $2 \mathrm{~nm}$ and serves as a guide to the eye for the interface sharpness.

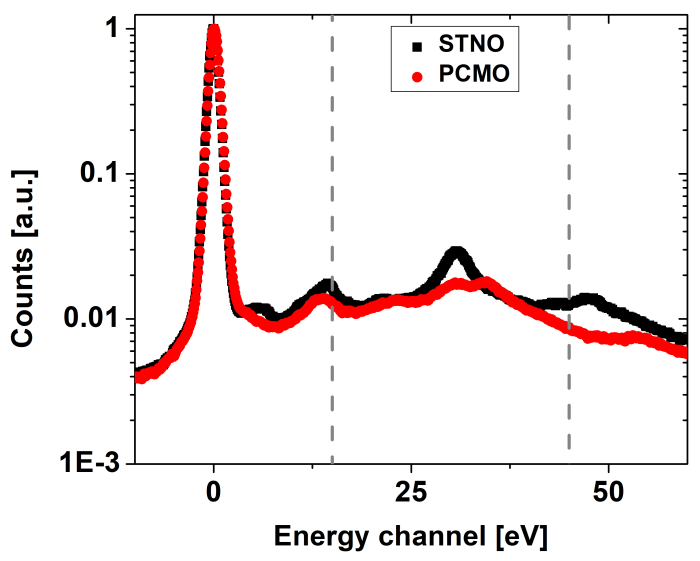

Figure A.17: Exemplary EEL spectra of STNO and PCMO with the fitting range used in Eq. (7.3) marked by grey dashed lines. 


\section{A.16 Atomically Resolved EBIC: Domain Assign- ment}
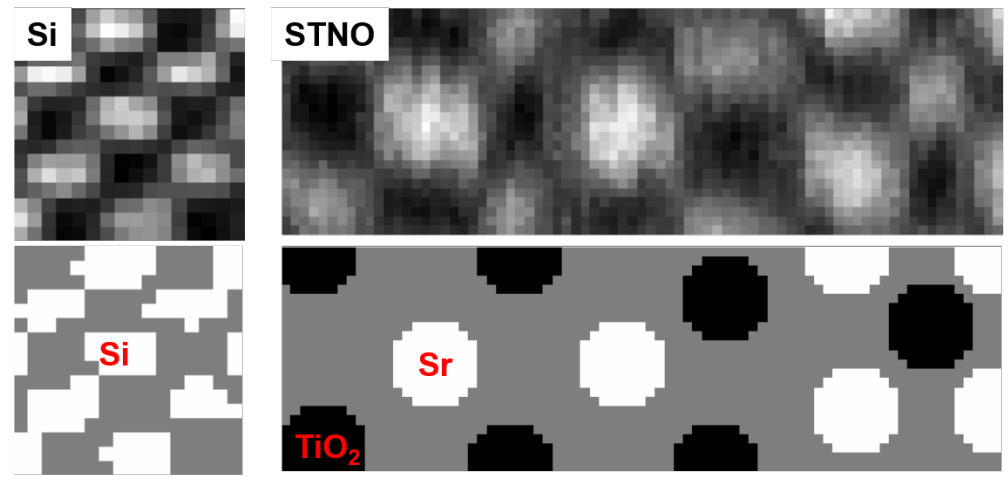

Figure A.18: Domain assignment for the analysis of atomically modulating EBIC signals on the basis ADF images (top): In the case of Si, all values above resp. below the mean $\mathrm{ADF}$ intensity are assigned to atomic sites resp. gaps. In the case of STNO, the atomic positions are localised as local maxima after Gaussian convolution and pixels with a distance smaller than a quarter of the lattice constant are assigned to either $\mathrm{Sr}$ or $\mathrm{TiO}_{2}$ columns. 


\section{Bibliography}

[1] L. C. Hamilton and M. D. Stampone, "Blowin'in the wind: Shortterm weather and belief in anthropogenic climate change," Weather, Climate, and Society, vol. 5, no. 2, pp. 112-119, 2013.

[2] N. Oreskes, "The scientific consensus on climate change," Science, vol. 306, no. 5702, pp. 1686-1686, 2004.

[3] J. L. Sarmiento, T. M. Hughes, R. J. Stouffer, and S. Manabe, "Simulated response of the ocean carbon cycle to anthropogenic climate warming," Nature, vol. 393, no. 6682, pp. 245-249, 1998.

[4] T. P. Barnett, D. W. Pierce, and R. Schnur, "Detection of anthropogenic climate change in the world's oceans," Science, vol. 292, no. 5515, pp. 270-274, 2001.

[5] C. Rosenzweig, D. Karoly, M. Vicarelli, P. Neofotis, Q. Wu, G. Casassa, A. Menzel, T. L. Root, N. Estrella, B. Seguin, et al., "Attributing physical and biological impacts to anthropogenic climate change," Nature, vol. 453, no. 7193, pp. 353-357, 2008.

[6] M. R. Allen, P. A. Stott, J. F. Mitchell, R. Schnur, and T. L. Delworth, "Quantifying the uncertainty in forecasts of anthropogenic climate change," Nature, vol. 407, no. 6804, pp. 617-620, 2000.

[7] A. Wiin-Nielsen, "The increased greenhouse effect-A general review," Proceedings of the Indian Academy of Sciences-Earth and Planetary Sciences, vol. 102, no. 1, pp. 3-25, 1993.

[8] C. Maucieri, A. C. Barbera, J. Vymazal, and M. Borin, "A review on the main affecting factors of greenhouse gases emission in constructed wetlands," Agricultural and forest meteorology, vol. 236, pp. 175-193, 2017. 
[9] J. Guerrero, D. Gebbran, S. Mhanna, A. C. Chapman, and G. Verbič, "Towards a transactive energy system for integration of distributed energy resources: Home energy management, distributed optimal power flow, and peer-to-peer energy trading," Renewable and Sustainable Energy Reviews, vol. 132, p. 110000, 2020.

[10] P. K. Nayak, S. Mahesh, H. J. Snaith, and D. Cahen, "Photovoltaic solar cell technologies: analysing the state of the art," Nature Reviews Materials, vol. 4, no. 4, p. 269, 2019.

[11] https://www.ise.fraunhofer.de/content/dam/ise/de/ documents/publications/studies/Photovoltaics-Report.pdf, $27 / 07 / 2020$.

[12] W. Shockley and H. J. Queisser, "Detailed balance limit of efficiency of p-n junction solar cells," Journal of applied physics, vol. 32, no. 3, pp. 510-519, 1961.

[13] W. S. Yang, J. H. Noh, N. J. Jeon, Y. C. Kim, S. Ryu, J. Seo, and S. I. Seok, "High-performance photovoltaic perovskite layers fabricated through intramolecular exchange," Science, vol. 348, no. 6240, pp. 1234-1237, 2015.

[14] M. Abdi-Jalebi, Z. Andaji-Garmaroudi, S. Cacovich, C. Stavrakas, B. Philippe, J. M. Richter, M. Alsari, E. P. Booker, E. M. Hutter, A. J. Pearson, et al., "Maximizing and stabilizing luminescence from halide perovskites with potassium passivation," Nature, vol. 555, no. 7697, pp. 497-501, 2018.

[15] H. J. Snaith, "Present status and future prospects of perovskite photovoltaics," Nature materials, vol. 17, no. 5, pp. 372-376, 2018.

[16] B. Ifland, P. Peretzki, B. Kressdorf, P. Saring, A. Kelling, M. Seibt, and C. Jooss, "Current-voltage characteristics of manganite-titanite perovskite junctions," Beilstein journal of nanotechnology, vol. 6, no. 1, pp. 1467-1484, 2015.

[17] B. Ifland, J. Hoffmann, B. Kressdorf, V. Roddatis, M. Seibt, and C. Jooss, "Contribution of Jahn-Teller and charge transfer excitations to the photovoltaic effect of manganite/titanite heterojunctions," New Journal of Physics, vol. 19, no. 6, p. 063046, 2017. 
[18] D. Raiser, S. Mildner, B. Ifland, M. Sotoudeh, P. Blöchl, S. Techert, and C. Jooss, "Evolution of hot polaron states with a nanosecond lifetime in a manganite perovskite," Advanced energy materials, vol. 7, no. 12, p. $1602174,2017$.

[19] E. Pollert, S. Krupicka, and E. Kuzmicova, "Structural study of Pr 1-x $\mathrm{Ca}$ x MnO 3 and Y 1-x Ca x MnO 3 perovskites," Journal of Physics and Chemistry of Solids, vol. 43, no. 12, pp. 1137-1145, 1982.

[20] Z. Jirak, S. Krupička, Z. Šimša, M. Dlouha, and S. Vratislav, "Neutron diffraction study of $\operatorname{Pr} 1-\mathrm{x} \mathrm{Ca} \times \mathrm{MnO} 3$ perovskites," Journal of magnetism and magnetic materials, vol. 53, no. 1-2, pp. 153-166, 1985.

[21] J. B. Goodenough, Magnetism and chemical bond, vol. 1. Interscience Publ., 1963.

[22] R. von Helmolt, J. Wecker, B. Holzapfel, L. Schultz, and K. Samwer, "Giant negative magnetoresistance in perovskitelike La $2 / 3 \mathrm{Ba} 1 / 3$ $\mathrm{MnO}$ x ferromagnetic films," Physical Review Letters, vol. 71, no. 14, p. 2331, 1993.

[23] S. Raabe, D. Mierwaldt, J. Ciston, M. Uijttewaal, H. Stein, J. Hoffmann, Y. Zhu, P. Blöchl, and C. Jooss, "In situ electrochemical electron microscopy study of oxygen evolution activity of doped manganite perovskites," Advanced Functional Materials, vol. 22, no. 16, pp. 33783388, 2012.

[24] D. Mierwaldt, S. Mildner, R. Arrigo, A. Knop-Gericke, E. Franke, A. Blumenstein, J. Hoffmann, and C. Jooss, "In situ XANES/XPS investigation of doped manganese perovskite catalysts," Catalysts, vol. 4, no. 2, pp. 129-145, 2014.

[25] S. Mildner, M. Beleggia, D. Mierwaldt, T. W. Hansen, J. B. Wagner, S. Yazdi, T. Kasama, J. Ciston, Y. Zhu, and C. Jooss, "Environmental TEM study of electron beam induced electrochemistry of Pr 0.64 Ca $0.36 \mathrm{MnO} 3$ catalysts for oxygen evolution," The Journal of Physical Chemistry C, vol. 119, no. 10, pp. 5301-5310, 2015.

[26] S. Lashkare, S. Chouhan, T. Chavan, A. Bhat, P. Kumbhare, and U. Ganguly, "Pcmo rram for integrate-and-fire neuron in spiking neural networks," IEEE Electron Device Letters, vol. 39, no. 4, pp. 484487, 2018. 
[27] B. Kressdorf, T. Meyer, A. Belenchuk, O. Shapoval, M. Ten Brink, S. Melles, U. Ross, J. Hoffmann, V. Moshnyaga, M. Seibt, et al., "Room-Temperature Hot-Polaron Photovoltaics in the ChargeOrdered State of a Layered Perovskite Oxide Heterojunction," Physical Review Applied, vol. 14, no. 5, p. 054006, 2020.

[28] B. Kressdorf, T. Meyer, M. ten Brink, C. Seick, S. Melles, N. Ottinger, T. Titze, H. Meer, A. Weisser, J. Hoffmann, et al., "Orbital-order phase transition in $\operatorname{Pr} 1-\mathrm{x} \mathrm{Ca} \times \mathrm{MnO} 3$ probed by photovoltaics," Physical Review B, vol. 103, no. 23, p. 235122, 2021.

[29] P. Peretzki, Implementation and Quantification of Scanning Transmission EBIC Experiments for Measuring Nanometer Diffusion Lengths in Manganite-titanite Pn Heterojunctions. PhD thesis, Georg-AugustUniversität Göttingen, 2019.

[30] X. Liu, T. Man, J. Yin, X. Lu, S. Guo, T. Ohmura, and D. Ping, "In situ heating TEM observations on carbide formation and $\alpha$-Fe recrystallization in twinned martensite," Scientific reports, vol. 8, no. 1, pp. 1-9, 2018.

[31] W. Yuan, B. Zhu, X.-Y. Li, T. W. Hansen, Y. Ou, K. Fang, H. Yang, Z. Zhang, J. B. Wagner, Y. Gao, et al., "Visualizing H2O molecules reacting at $\mathrm{TiO} 2$ active sites with transmission electron microscopy," Science, vol. 367, no. 6476, pp. 428-430, 2020.

[32] B. L. Nannenga, D. Shi, A. G. Leslie, and T. Gonen, "High-resolution structure determination by continuous-rotation data collection in MicroED," Nature methods, vol. 11, no. 9, pp. 927-930, 2014.

[33] F. M. Ross and A. M. Minor, "In situ transmission electron microscopy," in Springer Handbook of Microscopy, pp. 2-2, Springer, 2019.

[34] C. B. Carter and D. B. Williams, Transmission electron microscopy: Diffraction, imaging, and spectrometry. Springer, 2016.

[35] R. F. Egerton, Electron energy-loss spectroscopy in the electron microscope. Springer Science \& Business Media, 2011.

[36] C. Ophus, "Four-dimensional scanning transmission electron microscopy (4D-STEM): From scanning nanodiffraction to ptychography and beyond," Microscopy and Microanalysis, vol. 25, no. 3, pp. 563$582,2019$. 
[37] G. Rose, "Beschreibung einiger neuen Mineralien des Urals," Annalen der Physik, vol. 124, no. 12, pp. 551-573, 1839.

[38] V. M. Goldschmidt, "Die gesetze der krystallochemie," Naturwissenschaften, vol. 14, no. 21, pp. 477-485, 1926.

[39] H. Hwang, S.-W. Cheong, P. Radaelli, M. Marezio, and B. Batlogg, "Lattice Effects on the Magnetoresistance in Doped LaMnO 3," Physical review letters, vol. 75, no. 5, p. 914, 1995.

[40] Z. Shengming, Z. Hong, S. Lei, Z. Zongyan, Z. Guien, and Z. Yuheng, "The effect of cation disorder on electrical transport properties of Nd 0.7 (Ca, Sr, Ba) 0.3 MnO 3," Journal of Physics: Condensed Matter, vol. 11, no. 36 , p. $6877,1999$.

[41] A. Glazer, "The classification of tilted octahedra in perovskites," Acta Crystallographica Section B: Structural Crystallography and Crystal Chemistry, vol. 28, no. 11, pp. 3384-3392, 1972.

[42] E. Salje, M. Gallardo, J. Jiménez, F. Romero, and J. Del Cerro, "The cubic-tetragonal phase transition in strontium titanate: excess specific heat measurements and evidence for a near-tricritical, mean field type transition mechanism," Journal of Physics: Condensed Matter, vol. 10, no. 25, p. 5535, 1998.

[43] G. Jones and P. Thomas, "The tetragonal phase of Na 0.5 Bi 0.5 TiO 3-a new variant of the perovskite structure," Acta Crystallographica Section B: Structural Science, vol. 56, no. 3, pp. 426-430, 2000.

[44] A. Hewat, "Cubic-tetragonal-orthorhombic-rhombohedral ferroelectric transitions in perovskite potassium niobate: neutron powder profile refinement of the structures," Journal of Physics C: Solid State Physics, vol. 6, no. 16, p. 2559, 1973.

[45] B. Noheda, J. Gonzalo, L. Cross, R. Guo, S.-E. Park, D. Cox, and G. Shirane, "Tetragonal-to-monoclinic phase transition in a ferroelectric perovskite: The structure of PbZr 0.52 Ti 0.48 O 3," Physical Review B, vol. 61, no. 13, p. 8687, 2000.

[46] K.-I. Kobayashi, T. Kimura, H. Sawada, K. Terakura, and Y. Tokura, "Room-temperature magnetoresistance in an oxide material with an ordered double-perovskite structure," Nature, vol. 395, no. 6703, pp. 677-680, 1998. 
[47] S. Ruddlesden and P. Popper, "New compounds of the K 2 NiF 4 type," Acta Crystallographica, vol. 10, no. 8, pp. 538-539, 1957.

[48] P. M. Woodward, "Octahedral tilting in perovskites. I. Geometrical considerations," Acta Crystallographica Section B: Structural Science, vol. 53, no. 1, pp. 32-43, 1997.

[49] J.-S. Zhou and J. B. Goodenough, "Universal octahedral-site distortion in orthorhombic perovskite oxides," Physical review letters, vol. 94, no. 6, p. 065501, 2005.

[50] R. Tamazyan and S. Van Smaalen, "Quantitative description of the tilt of distorted octahedra in ABX 3 structures," Acta Crystallographica Section B: Structural Science, vol. 63, no. 2, pp. 190-200, 2007.

[51] D. Sánchez, J. Alonso, and M. Martínez-Lope, "Neutron-diffraction study of the jahn-teller transition in PrMnO 3," Journal of the Chemical Society, Dalton Transactions, no. 23, pp. 4422-4425, 2002.

[52] Z. Jirák, J. Hejtmánek, K. Knızek, and R. Sonntag, "Structure and Properties of the Pr 1-x K x MnO 3 Perovskites (x=0-0.15)," Journal of Solid State Chemistry, vol. 132, no. 1, pp. 98-106, 1997.

[53] H. A. Jahn and E. Teller, "Stability of polyatomic molecules in degenerate electronic states-I-Orbital degeneracy," Proceedings of the Royal Society of London. Series A-Mathematical and Physical Sciences, vol. 161, no. 905, pp. 220-235, 1937.

[54] M. Sotoudeh, S. Rajpurohit, P. Blöchl, D. Mierwaldt, J. Norpoth, V. Roddatis, S. Mildner, B. Kressdorf, B. Ifland, and C. Jooss, "Electronic structure of Pr 1-x Ca x MnO 3," Physical Review B, vol. 95, no. 23, p. 235150, 2017.

[55] J.-S. Zhou and J. B. Goodenough, "Orbital order-disorder transition in single-valent manganites," Physical Review B, vol. 68, no. 14, p. 144406, 2003.

[56] S. Rajpurohit, The study of magnetic and polaronic microstructure in $\operatorname{Pr} 1-x \mathrm{Ca} x \mathrm{MnO} 3$ manganite series. dissertation, Georg-AugustUniversität Göttingen, 2018.

[57] L. Wu, R. Klie, Y. Zhu, and C. Jooss, "Experimental confirmation of Zener-polaron-type charge and orbital ordering in $\operatorname{Pr} 1-\mathrm{x} \mathrm{Ca} \times \mathrm{MnO}$ 3," Physical Review B, vol. 76, no. 17, p. 174210, 2007. 
[58] P. A. Cox, Transition metal oxides: an introduction to their electronic structure and properties, vol. 27. Oxford university press, 2010.

[59] C. Bi, J. Ma, J. Yan, X. Fang, B. Zhao, D. Yao, and X. Qiu, "Electronphonon coupling in Nb-doped SrTiO 3 single crystal," Journal of Physics: Condensed Matter, vol. 18, no. 8, p. 2553, 2006.

[60] S. Yamada, T.-h. Arima, H. Ikeda, and K. Takita, "Thermopower in Pr 1-x Ca x MnO 3," Journal of the Physical Society of Japan, vol. 69, no. 5, pp. 1278-1281, 2000.

[61] S. Mildner, J. Hoffmann, P. Blöchl, S. Techert, and C. Jooss, "Temperature-and doping-dependent optical absorption in the smallpolaron system Pr 1-x Ca x MnO 3," Physical Review B, vol. 92, no. 3, p. $035145,2015$.

[62] G. Saucke, J. Norpoth, C. Jooss, D. Su, and Y. Zhu, "Polaron absorption for photovoltaic energy conversion in a manganite-titanate pn heterojunction," Physical Review B, vol. 85, no. 16, p. 165315, 2012.

[63] A. Yakimov, A. Dvurechenskii, Y. Y. Proskuryakov, A. Nikiforov, O. Pchelyakov, S. Teys, and A. Gutakovskii, "Normal-incidence infrared photoconductivity in $\mathrm{Si}$ pin diode with embedded Ge selfassembled quantum dots," Applied physics letters, vol. 75, no. 10, pp. 1413-1415, 1999.

[64] J. Guo, F.-M. Pan, M. Feng, R. Guo, P. Chou, and C. Chang, "Schottky contact and the thermal stability of Ni on n-type GaN," Journal of applied physics, vol. 80, no. 3, pp. 1623-1627, 1996.

[65] S. M. Sze and K. K. Ng, Physics of semiconductor devices. John wiley \& sons, 2006.

[66] R. L. Anderson, "Experiments on Ge-GaAs heterojunctions," in Electronic Structure of Semiconductor Heterojunctions, pp. 35-48, Springer, 1988.

[67] W. Shockley, "The theory of p-n junctions in semiconductors and p-n junction transistors," Bell System Technical Journal, vol. 28, no. 3, pp. 435-489, 1949.

[68] L. Schmidt-Mende and J. Weickert, Organic and hybrid solar cells: an introduction. Walter de Gruyter GmbH \& Co KG, 2016. 
[69] V. Kveder, M. Kittler, and W. Schröter, "Recombination activity of contaminated dislocations in silicon: A model describing electronbeam-induced current contrast behavior," Physical Review B, vol. 63, no. 11, p. 115208, 2001.

[70] P. Würfel, Physik der Solarzellen. Spektrum, Akad. Verlag, 2000.

[71] M. A. Green, "Radiative efficiency of state-of-the-art photovoltaic cells," Progress in Photovoltaics: Research and Applications, vol. 20, no. 4, pp. 472-476, 2012.

[72] M. Von Ardenne, "Das Elektronen-Rastermikroskop. Praktische Ausführung," Zeitschrift für technische Physik, vol. 19, no. 11, pp. 407-416, 1938.

[73] L. Reimer, Scanning electron microscopy: physics of image formation and microanalysis, vol. 45. Springer, 2013.

[74] A. Crewe, D. Eggenberger, J. Wall, and L. Welter, "Electron gun using a field emission source," Review of Scientific Instruments, vol. 39, no. 4, pp. 576-583, 1968.

[75] G. E. Lloyd, "Atomic number and crystallographic contrast images with the SEM: a review of backscattered electron techniques," Mineralogical Magazine, vol. 51, no. 359, pp. 3-19, 1987.

[76] G. Danilatos, "Review and outline of environmental SEM at present," Journal of microscopy, vol. 162, no. 3, pp. 391-402, 1991.

[77] W. Escovitz, T. Fox, and R. Levi-Setti, "Scanning transmission ion microscope with a field ion source," Proceedings of the National Academy of Sciences, vol. 72, no. 5, pp. 1826-1828, 1975.

[78] L. A. Giannuzzi et al., Introduction to focused ion beams: instrumentation, theory, techniques and practice. Springer Science \& Business Media, 2004.

[79] L. A. Giannuzzi and F. A. Stevie, "A review of focused ion beam milling techniques for TEM specimen preparation," Micron, vol. 30, no. 3, pp. 197-204, 1999.

[80] D. Barr and W. Brown, "Contrast formation in focused ion beam images of polycrystalline aluminum," Journal of Vacuum Science \& Technology B: Microelectronics and Nanometer Structures Processing, Measurement, and Phenomena, vol. 13, no. 6, pp. 2580-2583, 1995. 
[81] T. Everhart, Contrast formation in the scanning electron microscope. PhD thesis, Cambridge University, 1958.

[82] H. Leamy, "Charge collection scanning electron microscopy," Journal of Applied Physics, vol. 53, no. 6, pp. R51-R80, 1982.

[83] T. Everhart and P. Hoff, "Determination of kilovolt electron energy dissipation vs penetration distance in solid materials," Journal of Applied Physics, vol. 42, no. 13, pp. 5837-5846, 1971.

[84] C. Donolato, "Reconstruction of the charge collection probability in a semiconductor device from the derivative of collection efficiency data," Applied Physics Letters, vol. 75, no. 25, pp. 4004-4006, 1999.

[85] C. C. Tan, V. K. Ong, and K. Radhakrishnan, "The study of the charge collection of the normal-collector configuration," Progress in Photovoltaics: Research and Applications, vol. 21, no. 5, pp. 986-995, 2013.

[86] P. M. Haney, H. P. Yoon, B. Gaury, and N. B. Zhitenev, "Depletion region surface effects in electron beam induced current measurements," Journal of applied physics, vol. 120, no. 9, p. 095702, 2016.

[87] J. E. Moore, C. A. Affouda, S. I. Maximenko, and P. Jenkins, "Analytical and numerical simulation of electron beam induced current profiles in pn junctions," Journal of Applied Physics, vol. 124, no. 11, p. 113102, 2018.

[88] H. Kuiken, "Theory of lifetime measurements with the scanning electron microscope: Transient analysis," Solid-State Electronics, vol. 19, no. 6, pp. 447-450, 1976.

[89] C. Donolato, "Evaluation of diffusion lengths and surface recombination velocities from electron beam induced current scans," Applied Physics Letters, vol. 43, no. 1, pp. 120-122, 1983.

[90] M. Knoll and E. Ruska, "Das Elektronenmikroskop," Zeitschrift für physik, vol. 78, no. 5-6, pp. 318-339, 1932.

[91] E. Abbe, "Beiträge zur Theorie des Mikroskops und der mikroskopischen Wahrnehmung," Archiv für mikroskopische Anatomie, vol. 9, no. 1, pp. 413-468, 1873. 
[92] M. Haider, S. Uhlemann, E. Schwan, H. Rose, B. Kabius, and K. Urban, "Electron microscopy image enhanced," Nature, vol. 392, no. 6678, pp. 768-769, 1998.

[93] C. Jia, M. Lentzen, and K. Urban, "Atomic-resolution imaging of oxygen in perovskite ceramics," Science, vol. 299, no. 5608, pp. 870 $873,2003$.

[94] O. Krivanek, N. Dellby, and A. Lupini, "Towards sub- $\AA$ electron beams," Ultramicroscopy, vol. 78, no. 1-4, pp. 1-11, 1999.

[95] J. I. Goldstein, D. E. Newbury, J. R. Michael, N. W. Ritchie, J. H. J. Scott, and D. C. Joy, Scanning electron microscopy and X-ray microanalysis. Springer, 2017.

[96] S. J. Pennycook and P. D. Nellist, Scanning transmission electron microscopy: imaging and analysis. Springer Science \& Business Media, 2011.

[97] T. Malis, S. Cheng, and R. Egerton, "EELS log-ratio technique for specimen-thickness measurement in the TEM," Journal of electron microscopy technique, vol. 8, no. 2, pp. 193-200, 1988.

[98] G. Cliff and G. Lorimer, "The quantitative analysis of thin specimens," Journal of Microscopy, vol. 103, no. 2, pp. 203-207, 1975.

[99] G. Brunetti, D. Robert, P. Bayle-Guillemaud, J. Rouviere, E. Rauch, J. Martin, J. Colin, F. Bertin, and C. Cayron, "Confirmation of the domino-cascade model by LiFePO 4/FePO 4 precession electron diffraction," Chemistry of Materials, vol. 23, no. 20, pp. 4515-4524, 2011.

[100] A. Kobler, A. Kashiwar, H. Hahn, and C. Kübel, "Combination of in situ straining and ACOM TEM: A novel method for analysis of plastic deformation of nanocrystalline metals," Ultramicroscopy, vol. 128, pp. 68-81, 2013.

[101] V. Ozdol, C. Gammer, X. Jin, P. Ercius, C. Ophus, J. Ciston, and A. Minor, "Strain mapping at nanometer resolution using advanced nano-beam electron diffraction," Applied Physics Letters, vol. 106, no. 25, p. 253107, 2015. 
[102] C. Gammer, C. Ophus, T. C. Pekin, J. Eckert, and A. M. Minor, "Local nanoscale strain mapping of a metallic glass during in situ testing," Applied Physics Letters, vol. 112, no. 17, p. 171905, 2018.

[103] P. Voyles and D. Muller, "Fluctuation microscopy in the STEM," Ultramicroscopy, vol. 93, no. 2, pp. 147-159, 2002.

[104] K. Müller, F. F. Krause, A. Béché, M. Schowalter, V. Galioit, S. Löffler, J. Verbeeck, J. Zweck, P. Schattschneider, and A. Rosenauer, "Atomic electric fields revealed by a quantum mechanical approach to electron picodiffraction," Nature communications, vol. 5, no. 1, pp. 1-8, 2014.

[105] M. Krajnak, D. McGrouther, D. Maneuski, V. O'Shea, and S. McVitie, "Pixelated detectors and improved efficiency for magnetic imaging in STEM differential phase contrast," Ultramicroscopy, vol. 165, pp. $42-$ $50,2016$.

[106] N. J. Zaluzec, "Computationally mediated experimental science," Microscopy and Microanalysis, vol. 9, no. S02, pp. 150-151, 2003.

[107] A. Clausen, D. Weber, K. Ruzaeva, V. Migunov, A. Baburajan, A. Bahuleyan, J. Caron, R. Chandra, S. Dey, S. Halder, B. D. Levin, M. Nord, C. Ophus, S. Peter, J. Schyndel van, J. Shin, S. Sunku, K. Müller-Caspary, and R. E. Dunin-Borkowski, "Libertem/libertem: 0.5.0."

[108] B. H. Savitzky, L. Hughes, K. C. Bustillo, H. D. Deng, N. L. Jin, E. G. Lomeli, W. C. Chueh, P. Herring, A. Minor, and C. Ophus, "py4DSTEM: Open Source Software for 4D-STEM Data Analysis," Microscopy and Microanalysis, vol. 25, no. S2, pp. 124-125, 2019.

[109] S. Somnath, C. R. Smith, S. Jesse, and N. Laanait, "Pycroscopy-An Open Source Approach to Microscopy and Microanalysis in the Age of Big Data and Open Science," Microscopy and Microanalysis, vol. 23, no. S1, pp. 224-225, 2017.

[110] L. Marton, "La microscopie electronique des objets biologiques," Bull. Acad. Roy. Med. Belg, vol. 21, pp. 600-617, 1935.

[111] v. E. Ruska, "Beitrag zur übermikroskopischen Abbildung bei höheren Drucken," Kolloid-Zeitschrift, vol. 100, no. 2, pp. 212-219, 1942. 
[112] H. G. Heide, "Electron microscopic observation of specimens under controlled gas pressure," The Journal of cell biology, vol. 13, no. 1, pp. $147-152,1962$.

[113] J. Park, H. Park, P. Ercius, A. F. Pegoraro, C. Xu, J. W. Kim, S. H. Han, and D. A. Weitz, "Direct observation of wet biological samples by graphene liquid cell transmission electron microscopy," Nano letters, vol. 15 , no. 7 , pp. 4737-4744, 2015.

[114] K. Svensson, Y. Jompol, H. Olin, and E. Olsson, "Compact design of a transmission electron microscope-scanning tunneling microscope holder with three-dimensional coarse motion," Review of scientific instruments, vol. 74, no. 11, pp. 4945-4947, 2003.

[115] H. H. Pérez Garza, K. Zuo, Y. Pivak, D. Morsink, M. Zakhozheva, M. Pen, S. van Weperen, and Q. Xu, "MEMS-based system for insitu biasing and heating solutions inside the TEM," in European Microscopy Congress 2016: Proceedings, pp. 237-238, Wiley Online Library, 2016.

[116] https://www.gatan.com/products/tem-specimen-holders, 04/06/2020.

[117] A. Twitchett, R. Dunin-Borkowski, and P. Midgley, "Quantitative electron holography of biased semiconductor devices," Physical review letters, vol. 88, no. 23, p. 238302, 2002.

[118] D. Wolf, A. Lubk, A. Lenk, S. Sturm, and H. Lichte, "Tomographic investigation of fermi level pinning at focused ion beam milled semiconductor surfaces," Applied Physics Letters, vol. 103, no. 26, p. 264104, 2013.

[119] J. B. Park, T. Niermann, D. Berger, A. Knauer, I. Koslow, M. Weyers, M. Kneissl, and M. Lehmann, "Impact of electron irradiation on electron holographic potentiometry," Applied Physics Letters, vol. 105, no. 9, p. 094102, 2014.

[120] W. Van Roosbroeck, "Injected current carrier transport in a semiinfinite semiconductor and the determination of lifetimes and surface recombination velocities," Journal of Applied Physics, vol. 26, no. 4, pp. 380-391, 1955. 
[121] D. A. Ehrlich, Two-Dimensional Finite Element Simulations of Electron Beam Induced Current. bachelor thesis, Georg-AugustUniversität Göttingen, 2019.

[122] D. Taylor, "Electron-beam-induced conductivity in thin insulating films," Radiation Physics and Chemistry (1977), vol. 13, no. 5-6, pp. 209-221, 1979.

[123] M. Mecklenburg, W. A. Hubbard, J. J. Lodico, and B. Regan, "Electron beam-induced current imaging with two-angstrom resolution," Ultramicroscopy, vol. 207, p. 112852, 2019.

[124] W. Hubbard, Z. Lingley, J. Theiss, S. Sitzman, T. Ayvazian, M. Brodie, and B. Foran, "Scanning transmission electron microscope mapping of electronic transport in polycrystalline BaTiO 3 ceramic capacitors," Applied Physics Letters, vol. 115, no. 13, p. 133502, 2019.

[125] T. Meyer, T. Westphal, B. Kressdorf, U. Ross, C. Jooss, and M. Seibt, "Site-specific plan-view TEM lamella preparation of pristine surfaces with a large field of view," Ultramicroscopy, p. 113320, 2021.

[126] T. P. Almeida, D. McGrouther, Y. Pivak, H. H. P. Garza, R. Temple, J. Massey, C. H. Marrows, and S. McVitie, "Preparation of highquality planar FeRh thin films for in situ TEM investigations," in Journal of Physics: Conference Series, vol. 903, p. 012022, IOP Publishing, 2017.

[127] C. Flathmann, H. Spende, T. Meyer, P. Peretzki, and M. Seibt, "Preparation Techniques for Cross-Section Transmission Electron Microscopy Lamellas Suitable for Investigating In Situ Silicon-Aluminum Alloying at Grain Boundaries in Multicrystalline Silicon," physica status solidi (a), vol. 216, no. 17, p. 1900308, 2019.

[128] S. E. Zeltmann, A. Müller, K. C. Bustillo, B. Savitzky, L. Hughes, A. M. Minor, and C. Ophus, "Patterned probes for high precision 4DSTEM bragg measurements," Ultramicroscopy, vol. 209, p. 112890, 2020 .

[129] R. Shiloh, P.-H. Lu, R. Remez, A. H. Tavabi, G. Pozzi, R. E. DuninBorkowski, and A. Arie, "Nanostructuring of electron beams," Physica Scripta, vol. 94, no. 3, p. 034004, 2019. 
[130] C. Mahr, K. Müller-Caspary, T. Grieb, M. Schowalter, T. Mehrtens, F. F. Krause, D. Zillmann, and A. Rosenauer, "Theoretical study of precision and accuracy of strain analysis by nano-beam electron diffraction," Ultramicroscopy, vol. 158, pp. 38-48, 2015.

[131] T. Grieb, F. F. Krause, M. Schowalter, D. Zillmann, R. Sellin, K. Müller-Caspary, C. Mahr, T. Mehrtens, D. Bimberg, and A. Rosenauer, "Strain analysis from nano-beam electron diffraction: Influence of specimen tilt and beam convergence," Ultramicroscopy, vol. 190, pp. 45-57, 2018.

[132] G. Guzzinati, W. Ghielens, C. Mahr, A. Béché, A. Rosenauer, T. Calders, and J. Verbeeck, "Electron Bessel beam diffraction for precise and accurate nanoscale strain mapping," Applied Physics Letters, vol. 114, no. 24, p. 243501, 2019.

[133] L. Kovarik, A. Stevens, A. Liyu, and N. D. Browning, "Implementing an accurate and rapid sparse sampling approach for low-dose atomic resolution STEM imaging," Applied Physics Letters, vol. 109, no. 16, p. $164102,2016$.

[134] T. Meyer, B. Kressdorf, V. Roddatis, J. Hoffmann, C. Jooss, and M. Seibt, "Phase Transitions in a Perovskite Thin Film Studied by Environmental In Situ Heating Nano-Beam Electron Diffraction," Small Methods, vol. n/a, no. n/a, p. 2100464.

[135] J. Cowley, "Electron nanodiffraction: Progress and prospects," Microscopy, vol. 45, no. 1, pp. 3-10, 1996.

[136] J. Fatermans, A. den Dekker, K. Müller-Caspary, N. Gauquelin, J. Verbeeck, and S. Van Aert, "Atom column detection from simultaneously acquired ABF and ADF STEM images," Ultramicroscopy, p. 113046, 2020.

[137] K. Usuda, T. Mizuno, T. Tezuka, N. Sugiyama, Y. Moriyama, S. Nakaharai, and S.-i. Takagi, "Strain relaxation of strained-Si layers on SiGe-on-insulator (SGOI) structures after mesa isolation," MRS Online Proceedings Library Archive, vol. 738, 2002.

[138] M. J. Hÿtch and A. M. Minor, "Observing and measuring strain in nanostructures and devices with transmission electron microscopy," MRS bulletin, vol. 39, no. 2, pp. 138-146, 2014. 
[139] D. Cooper, T. Denneulin, N. Bernier, A. Béché, and J.-L. Rouvière, "Strain mapping of semiconductor specimens with nm-scale resolution in a transmission electron microscope," Micron, vol. 80, pp. 145-165, 2016.

[140] D. Cooper, J.-P. Barnes, J.-M. Hartmann, A. Béché, and J.-L. Rouviere, "Dark field electron holography for quantitative strain measurements with nanometer-scale spatial resolution," Applied Physics Letters, vol. 95, no. 5, p. 053501, 2009.

[141] J. Gibson and M. M. J. Treacy, "The effect of elastic relaxation on the local structure of lattice-modulated thin films," Ultramicroscopy, vol. 14, no. 4, pp. 345-349, 1984.

[142] J. Wortman and R. Evans, "Young's modulus, shear modulus, and Poisson's ratio in silicon and germanium," Journal of applied physics, vol. 36, no. 1, pp. 153-156, 1965.

[143] H. Seitz, M. Seibt, F. Baumann, K. Ahlborn, and W. Schröter, "Quantitative strain mapping using high-resolution electron microscopy," physica status solidi (a), vol. 150, no. 2, pp. 625-634, 1995.

[144] P. Potapov, "The experimental electron mean-free-path in Si under typical (S) TEM conditions," Ultramicroscopy, vol. 147, pp. 21-24, 2014.

[145] N. J. Zaluzec, "Quantitative measurements of magnetic vortices using position resolved diffraction in lorentz STEM," Microscopy and Microanalysis, vol. 8, no. S02, pp. 376-377, 2002.

[146] D. Cooper, "Off-axis electron holography for the measurement of active dopants in silicon semiconductor devices," Journal of Physics D: Applied Physics, vol. 49, no. 47, p. 474001, 2016.

[147] M. Wu and E. Spiecker, "Correlative micro-diffraction and differential phase contrast study of mean inner potential and subtle beamspecimen interaction," Ultramicroscopy, vol. 176, pp. 233-245, 2017.

[148] M. Gajdardziska-Josifovska, M. McCartney, W. De Ruijter, D. J. Smith, J. Weiss, and J.-M. Zuo, "Accurate measurements of mean inner potential of crystal wedges using digital electron holograms," Ultramicroscopy, vol. 50, no. 3, pp. 285-299, 1993. 
[149] M. Haider, A. Epstein, P. Jarron, and C. Boulin, "A versatile, software configurable multichannel STEM detector for angle-resolved imaging," Ultramicroscopy, vol. 54, no. 1, pp. 41-59, 1994.

[150] T. Seki, G. Sánchez-Santolino, R. Ishikawa, S. D. Findlay, Y. Ikuhara, and N. Shibata, "Quantitative electric field mapping in thin specimens using a segmented detector: Revisiting the transfer function for differential phase contrast," Ultramicroscopy, vol. 182, pp. 258-263, 2017.

[151] K. Müller-Caspary, F. F. Krause, F. Winkler, A. Béché, J. Verbeeck, S. Van Aert, and A. Rosenauer, "Comparison of first moment STEM with conventional differential phase contrast and the dependence on electron dose," Ultramicroscopy, vol. 203, pp. 95-104, 2019.

[152] M. Wu, A. Tafel, P. Hommelhoff, and E. Spiecker, "Determination of 3D electrostatic field at an electron nano-emitter," Applied Physics Letters, vol. 114, no. 1, p. 013101, 2019.

[153] M. Treacy and J. Gibson, "Variable coherence microscopy: A rich source of structural information from disordered materials," Acta Crystallographica Section A: Foundations of Crystallography, vol. 52, no. 2, pp. 212-220, 1996.

[154] P. Voyles, J. Gibson, and M. Treacy, "Fluctuation microscopy: a probe of atomic correlations in disordered materials," Microscopy, vol. 49, no. 2, pp. 259-266, 2000.

[155] H. Rose and C. Kisielowski, "On the Reciprocity of TEM and STEM," Microscopy and Microanalysis, vol. 11, no. S02, p. 2114, 2005.

[156] T. L. Daulton, K. S. Bondi, and K. F. Kelton, "Nanobeam diffraction fluctuation electron microscopy technique for structural characterization of disordered materials-Application to $\mathrm{Al} 88-\mathrm{x}$ Y 7 Fe 5 Ti x metallic glasses," Ultramicroscopy, vol. 110, no. 10, pp. 1279-1289, 2010.

[157] A. D. Robison, Intel@ Threading Building Blocks (TBB), pp. 955-964. Boston, MA: Springer US, 2011.

[158] M. Frigo and S. G. Johnson, "The design and implementation of FFTW3," 
[159] C. Sanderson and R. Curtin, "Armadillo: a template-based C++ library for linear algebra," Journal of Open Source Software, vol. 1, no. 2 , p. $26,2016$.

[160] T. Meyer, S. Lopatin, M. Seibt, and V. Roddatis, "A posteriori synchronization of scanning transmission electron microscopy signals with kilopixel per second acquisition rates," Ultramicroscopy, vol. 200, pp. 62-66, 2019.

[161] J. Tikkanen, S. Kauhala, H. Huhtinen, and P. Paturi, "Anomalous Thermal Expansion in (Pr, Ca) MnO3 Due to Orbital Ordering," Physics Procedia, vol. 75, pp. 475-481, 2015.

[162] J. Norpoth, S. Mildner, M. Scherff, J. Hoffmann, and C. Jooss, "In situ TEM analysis of resistive switching in manganite based thin-film heterostructures," Nanoscale, vol. 6, no. 16, pp. 9852-9862, 2014.

[163] M. v. Zimmermann, C. Nelson, J. Hill, D. Gibbs, M. Blume, D. Casa, B. Keimer, Y. Murakami, C.-C. Kao, C. Venkataraman, et al., "X-ray resonant scattering studies of orbital and charge ordering in $\operatorname{Pr} 1-\mathrm{x} \mathrm{Ca}$ x MnO 3," Physical Review B, vol. 64, no. 19, p. 195133, 2001.

[164] K. Momma and F. Izumi, "VESTA 3 for three-dimensional visualization of crystal, volumetric and morphology data," Journal of applied crystallography, vol. 44, no. 6, pp. 1272-1276, 2011.

[165] T. Meyer, B. Kressdorf, J. Lindner, P. Peretzki, V. Roddatis, C. Jooss, and M. Seibt, "High-resolution Scanning Transmission EBIC Analysis of Misfit Dislocations at Perovskite pn-Heterojunctions," in Journal of Physics: Conference Series, vol. 1190, p. 012009, IOP Publishing, 2019.

[166] S. Suzuki, T. Yamamoto, H. Suzuki, K. Kawaguchi, K. Takahashi, and Y. Yoshisato, "Fabrication and characterization of Ba 1-x K x BiO 3/Nb-doped SrTiO 3 all-oxide-type Schottky junctions," Journal of applied physics, vol. 81, no. 10, pp. 6830-6836, 1997.

[167] I. Chakraborty, A. Singh, P. Kumbhare, N. Panwar, and U. Ganguly, "Materials parameter extraction using analytical models in PCMO based RRAM," in 2015 73rd Annual Device Research Conference (DRC), pp. 87-88, IEEE, 2015. 
[168] D. Kan, R. Aso, H. Kurata, and Y. Shimakawa, "Research Update: Interface-engineered oxygen octahedral tilts in perovskite oxide heterostructures," APL materials, vol. 3, no. 6, p. 062302, 2015.

[169] T. Meyer, D. A. Ehrlich, P. Pichler, V. Titova, C. Flathmann, J. Schmidt, and M. Seibt, "Tracing the boron diffusion into a textured silicon solar cell by combining boron diffusion simulation with experimental and simulated scanning transmission electron beam induced current," arXiv:2109.00586v1, 2021.

[170] D. McPhail, "Applications of secondary ion mass spectrometry (SIMS) in materials science," Journal of materials science, vol. 41, no. 3, pp. 873-903, 2006.

[171] C. Noguera, D. Spanjaard, and J. Friedel, "Dynamic screening of core hole. I. Semiclassical model," Journal of Physics F: Metal Physics, vol. 9, no. 6, p. 1189, 1979.

[172] G. Laramore and W. Camp, "Plasmon model for final-state relaxation effects in connection with core-level excitations in metals," Physical Review B, vol. 9, no. 8, p. 3270, 1974.

[173] C. Bark, D. Felker, Y. Wang, Y. Zhang, H. Jang, C. Folkman, J. Park, S. Baek, H. Zhou, D. Fong, et al., "Tailoring a two-dimensional electron gas at the LaAlO 3/SrTiO 3 (001) interface by epitaxial strain," Proceedings of the National Academy of Sciences, vol. 108, no. 12, pp. 4720-4724, 2011.

[174] D. Jannis, K. Müller-Caspary, A. Béché, A. Oelsner, and J. Verbeeck, "Spectroscopic coincidence experiments in transmission electron microscopy," Applied Physics Letters, vol. 114, no. 14, p. 143101, 2019.

[175] T. E. Oliphant, A guide to NumPy, vol. 1. Trelgol Publishing USA, 2006 .

[176] S. Van Der Walt, S. C. Colbert, and G. Varoquaux, "The NumPy array: a structure for efficient numerical computation," Computing in Science \&s Engineering, vol. 13, no. 2, p. 22, 2011.

[177] F. de la Peña, E. Prestat, V. T. Fauske, P. Burdet, P. Jokubauskas, M. Nord, T. Ostasevicius, K. E. MacArthur, M. Sarahan, D. N. Johnstone, J. Taillon, J. Lähnemann, V. Migunov, A. Eljarrat, J. Caron, T. Aarholt, S. Mazzucco, M. Walls, T. Slater, F. Winkler, pquinn 
dls, B. Martineau, G. Donval, R. McLeod, E. R. Hoglund, I. Alxneit, D. Lundeby, T. Henninen, L. F. Zagonel, and A. Garmannslund, "hyperspy/hyperspy: HyperSpy v1.5.2," Sept. 2019.

[178] P. Virtanen, R. Gommers, T. E. Oliphant, M. Haberland, T. Reddy, D. Cournapeau, E. Burovski, P. Peterson, W. Weckesser, J. Bright, S. J. van der Walt, M. Brett, J. Wilson, K. Jarrod Millman, N. Mayorov, A. R. J. Nelson, E. Jones, R. Kern, E. Larson, C. Carey, İ. Polat, Y. Feng, E. W. Moore, J. Vand erPlas, D. Laxalde, J. Perktold, R. Cimrman, I. Henriksen, E. A. Quintero, C. R. Harris, A. M. Archibald, A. H. Ribeiro, F. Pedregosa, P. van Mulbregt, and SciPy Contributors, "SciPy 1.0: Fundamental Algorithms for Scientific Computing in Python," Nature Methods, vol. 17, pp. 261-272, 2020. 


\section{Ackowledgements}

Firstly, I wish to express my deepest gratitude to my supervisor, Prof. Dr. Michael Seibt, for giving me the opportunity of pursuing my doctorate in his group and, in particular, for being an always available, esteemed partner for discussions - both scientific as well as personal - largely contributing to the development of my career.

Secondly, I would like to thank Prof. Dr. Christian Jooß for being a strongly committed co-supervisor and also for his dedication as the speaker of the SFB 1073 creating an excellent environment for this work.

Furthermore, I wish to thank all further members of the examination board and particularly Prof. Dr. Peter Blöchl for joining my thesis advisory committee.

I am strongly indebted to the research group of Prof. Dr. Michael Seibt, in particular to Dr. Patrick Peretzki and Arne Ahrens as incredibly helpful senior members when I joined as well as all my subsequent office members for always creating a friendly and joyful ambience, i.e. Hendrik Spende, Julian Sickel, Tobias Westphal, Jonas Drünert, Karen Stroh, Christoph Flathmann, Hendrik Voigt, David Ehrlich, and Felix Ben Müller.

Additionally, I would like to express my gratitude to Birte Kressdorf, Dr. Benedikt Ifland, and Stephan Melles for an outstandingly fruitful collaboration in the B02 project of the SFB 1073 as well as Carmen Kaspar, Antje Spliethoff-Laiser and Dr. Jörg Hoffmann as invaluable organisers of the latter.

I am furthermore strongly indebted to Dr. Vladimir Roddatis, Matthias Hahn, Volker Radisch, Dr. Ulrich Roß, Tobias Schulz, and Jan Verhoeven for their help with experimental designs and setups.

In addition, I would like to thank all remaining co-authors of the herein included papers for their contribution - i.e. Jonas Lindner, Dr. Sergei Lopatin, Prof. Dr. Peter Pichler, Valeriya Titova, and Prof. Dr. Jan Schmidt - as well as Tobias Westphal, Christoph Flathmann, Michael ten Brink, and Hugo Lourenço-Martins for carefully proofreading the manuscript. 
As mentioned in the main text, a detailed list of 4D-STEM contributors shall be given to whom I am all strongly indebted: General advise and input in fruitful discussions was given by Dr. Vladimir Roddatis and Prof. Dr. Michael Seibt. Additionally, small teams were formed to optimise experimental strategies, conduct experiments, and assess the functionality of the software tool including Christoph Flathmann in the context of strain mapping, Dr. Ulrich Roß, Jonas Lindner, and Christoph Flathmann in the context of electric field mapping, as well as Hendrik Voigt, Dr. Lin Tian, Lukas Richert, and Christoph Flathmann in the context of fluctuation electron microscopy.

Furthermore, I would like to acknowledge the following software packages used in this thesis: VESTA [164], NumPy [175, 176], HyperSpy [177], SciPy [178].

Lastly, I wish to thank my family and friends for their support during the past years and particularly Annika Tretbar who guided me thoughtfully through the writing period coinciding with COVID-19-related social distancing. 